\title{
Observations and modeling of polar faculae on the Sun
}

\author{
Dissertation \\ zur Erlangung des Doktorgrades \\ der Mathematisch-Naturwissenschaftlichen Fakultäten \\ der Georg-August-Universität zu Göttingen
}

vorgelegt von

\section{Oleg Okunev}

aus Sankt Petersburg / Russland

Göttingen 2004 
D7

Referent: Prof. Dr. F. Kneer

Korreferent: Prof. Dr. S. Dreizler

Tag der mündlichen Prüfung: 16.09.2004 


\section{Contents}

Summary 5

$\begin{array}{lll}1 & \text { Introduction } & 7\end{array}$

2 Observations and Data Reduction $\quad \mathbf{1 1}$

2.1 One-dimensional spectrograms observed with Gregory-Coudé Telescope . 13

2.1.1 Standard data reduction . . . . . . . . . . . . . . . . 15

2.1 .2 Noise filtering . . . . . . . . . . . . . . . 18

2.1.3 Reduction of Stokes $V$ spectrograms . . . . . . . . . . . . . . . . 22

2.1 .4 Intensity maps . . . . . . . . . . . . . . 23

2.2 Two-dimensional spectra observed with Vacuum Tower Telescope . . . . 25

2.2.1 Fabry-Perot interferometer . . . . . . . . . . . . 25

2.2.2 Structure of observations . . . . . . . . . . . . . . 27

2.2.3 Spectral quality of $2 \mathrm{D}$ spectrograms . . . . . . . . . . . 28

2.2.4 Broadband speckle reconstruction . . . . . . . . . . . . . . . . . . . . 30

2.2.5 Reconstruction of narrowband images . . . . . . . . . . . . 32

2.2 .6 Stokes $V$ corrections $\ldots \ldots \ldots \ldots \ldots$

2.3 Signal recognition in polarimetric data $\ldots \ldots . \ldots . . \ldots 37$

2.4 Instrumental polarization . . . . . . . . . . . . . . 43

$\begin{array}{lll}3 & \text { Analysis of the observations } & 47\end{array}$

3.1 Photometric properties of polar faculae . . . . . . . . . . . . . . . 47

3.1.1 Brightness contrast, center-to-limb variation . . . . . . . . . . . 47

3.1 .2 Fine structure and size . . . . . . . . . . . . . 50

3.2 Time evolution . . . . . . . . . . . . . . . . 52

3.3 Spectropolarimetric properties of polar faculae . . . . . . . . . . 55

3.3.1 Line core intensities and line depressions . . . . . . . . . 55

3.3.2 Velocities in polar faculae . . . . . . . . . . . . 58

3.3.3 Amplitudes of the polarimetric signal . . . . . . . . . . . . 62

3.3 .4 Calibration of magnetograms . . . . . . . . . . . . . . . 64

3.4 Spatial distribution of the magnetic field in $\mathrm{PFs} \ldots \ldots$

3.5 Magnetic polarity of polar faculae . . . . . . . . . . . . . . . 70

3.6 Summary of the observational properties of PFs . . . . . . . . . . . 73 
4 Numerical modeling $\quad \mathbf{7 5}$

4.1 Construction of the model . . . . . . . . . . . . . . . . . 77

4.1.1 Basic assumptions: two-component static model . . . . . . . . 77

4.1.2 Model of a single magnetic flux tube . . . . . . . . . . . . 77

4.1 .3 Temperature stratification inside MFT . . . . . . . . . . . . . . . 79

4.1.4 Calculation of the atmospheric parameters . . . . . . . . . 81

4.1.5 Construction of the 3D box and multiple rays . . . . . . . . . 83

4.2 Radiative transfer in a magnetized atmosphere . . . . . . . . . . . . 86

4.2.1 Zeeman effect and Stokes vector . . . . . . . . . . . . 86

4.2.2 Radiative transfer equation and numerical solution . . . . . . . . 89

4.2.3 Spectral line synthesis and contribution functions . . . . . . . . . 90

4.3 Model calculations . . . . . . . . . . . . . . . . . . . 92

5 Results of numerical modeling and comparison with observations 95

5.1 Effect of many MFTs along the ray . . . . . . . . . . . . . . . . . 95

5.2 Effect of internal thermal model . . . . . . . . . . . . . . . 96

5.3 Spectropolarimetric profiles from synthetic faculae . . . . . . . . . 102

5.4 Spatial distribution and fine structure . . . . . . . . . . . . . . . . . . 104

5.5 Center-to-limb variation . . . . . . . . . . . . . . . 105

5.6 Apparent magnetic field strength . . . . . . . . . . . . . . . . . 107

5.7 Summary of results from modeling . . . . . . . . . . . . . . . . . . 109

6 Conclusions and outlook 111

$\begin{array}{ll}\text { Bibliography } & 113\end{array}$

\begin{tabular}{ll}
\hline Acknowledgements & 117
\end{tabular}

$\begin{array}{ll}\text { Lebenslauf } & 119\end{array}$ 


\section{Summary}

Faculae on the polar caps of the Sun, in short polar faculae (PFs) are a part of the global magnetism of this star. PFs take part in the solar activity cycle. Similar to sunspots they possess an 11-12 years periodicity, shifted, however, by 5-6 years with respect to sunspot activity. PFs belong to a class of small-scale magnetic structures in the solar photosphere and therefore their detailed study requires high resolution polarimetric observations. In this work the accent was made on the structure of polar faculae, which were studied by means of high resolution white light and spectropolarimetric observations and realistic numerical simulations of faculae structures at the solar limb.

Observations which form the basis of the study were obtained in the years 2001 2002. They consist of spectropolarimetric data (Stokes $I$ and $V$ ) taken in the Fe I 6301.5 and 6302.5 $\AA$ and Fe II 6149.3 $\AA$ lines with the Greogory-Coudé Telescope (GCT) and the Vacuum Tower Telescope (VTT) at the Observatorio del Teide on Tenerife. At the VTT, the "Göttingen" two-dimensional Fabry-Perot spectrometer was used. It allows image reconstruction with speckle methods resulting in spatial resolution of approximately 0.25 for broadband images and 0 '.5 for spectropolarimetric observations. The application of singular value decomposition to noise filtering of spectrograms and signal recognition in polarimetric data yielded a polarimetric detection limit of $|V| \approx 2 \times 10^{-3} I_{\mathrm{c}}$.

Observations reveal that PFs, having sizes of $1^{\prime \prime}$ or larger, possess substantial fine structure of both brightness and magnetic fields. From the white-light speckle reconstructed images we found that PFs exhibit very high contrast in the continuum, which in some cases exceeds $50 \%$ of the average brightness of the ambient photosphere.

The center-to-limb variation (CLV) of the intensity contrast was derived. Polar faculae have enhanced contrast at large heliocentric angles, which however monotonically decreases towards the extreme limb of the Sun. No maximum was found within the observed range of heliocentric angles $\theta \geq 66^{\circ}(\mu=\cos \theta \leq 0.4)$.

The lifetime of PFs exceeds the duration of observations of $\sim 1$ hour. Yet, at small scales polar faculae appear to be highly dynamical structures, the brightness and the location of tiny facular points changes noticeably within $50 \mathrm{~s}$.

Spectropolarimetric properties inherent to PFs (line core contrasts, depressions of Stokes $I$ and separations and amplitudes of Stokes $V$ in three spectral lines) were gathered, which strongly distinguish facula atmospheres from the surrounding. The properties can serve as observational constraints on numerical simulations.

Kilo-Gauss magnetic fields detected earlier in polar faculae are confirmed by applying several approaches of calibration of the magnetic field measurements. The facular points possess strong fields and are unipolar with the same polarity as the global, poloidal magnetic field of the Sun. The ambient areas, however, exhibit weak flux features of both polarities, as in the quiet Sun near disk center. 
From the line center displacements of Stokes $I$ and zero crossing of Stokes $V$ strong upflows of $0.5-1 \mathrm{~km} \mathrm{~s}^{-1}$ are detected in PFs. The finding of upflows in magnetic structures is surprising, because it is widely accepted that small-scale magnetic structures are located in the regions of downflows. According to estimations such upflows in PFs can feed the fast solar wind from polar coronal holes with sufficient mass.

To verify a hypothesis about PFs as a concentration of small-scale magnetic flux tubes a numerical simulation was performed. A semi-empirical two-component 3D model of faculae structures, controlled by several free parameters, was constructed. Multi-ray 1.5D radiative transfer calculations were performed along oblique rays going through a highly inhomogeneous atmosphere of the simulation box. By the comparison of the properties of the calculated Stokes profiles from the synthetic faculae with the observed properties, a set of free parameters of the model was deduced, which satisfy observational constraints. 


\section{Introduction}

Polar faculae (PFs) on the Sun appear as bright points or as bright objects with complex structure a few seconds of arc in diameter, on the north and south polar caps of the Sun, usually at heliographic latitude $\left|\phi_{\odot}\right| \geq 60^{\circ}$. They can be seen in white light, in Ca K and in some chromospheric lines. They are not grouped to form luminous patches like the faculae found at lower latitudes, but are scattered at random. PFs are small-scale structures and therefore good observational (seeing) conditions and high spatial resolution are essential for their detailed study.

Polar faculae are of special interest for solar physics because of their close relationship to the global magnetic field of the Sun and to solar activity. Solar magnetic field is responsible for much of the variability of the Sun on various time and spatial scales and it is generated by a magnetic dynamo within the Sun. A comprehensive review of solar dynamo was given recently by Ossendrijver (2003).

Together with sunspots (dark magnetic regions at low latitudes of the Sun) polar faculae take part in the magnetic cycle of the Sun and are an important aspect of solar magnetism (Waldmeier 1955, 1962; Sheeley 1964, 1991, Makarov et al. 2003 and references in these works). However, according to Makarov \& Makarova (1996), the PFs precede the sunspot cycle by 5-6 years. During sunspot minimum, they appear at the polar caps of the Sun at heliographic latitudes $\left|\phi_{\odot}\right| \geq 60^{\circ}$. During the growth of sunspot activity, this lower latitude boundary migrates towards the poles and the regions of PF appearance vanish during the maximum of sunspot activity (see, e.g., Makarov \& Sivaraman 1989). Wang \& Sheeley (2003) from numerical modeling describe the poleward migration of the lower latitude boundary as due to emergence and growth of bipolar regions at medium latitudes during the activity cycle.

When sunspot activity reaches its maximum and the polar activity is accordingly in the minimum, the Sun changes the polarity of its global poloidal magnetic field as shown in the latitude-time diagram (butterfly diagram) of solar magnetism (Fig. 1.1). The reversal of the magnetic polarity is not an instant process, it can take several months. The widely accepted mechanism of the polar fields reversal is based on a solar surface flux transport model (Wang \& Sheeley 2003, Dikpati et al. 2004). According to this scenario the trailing polarity from bipolar active regions drifts towards the poles, where it neutralizes the opposite polarity from the previous cycle. The reversal of the polar magnetic field occurs when sufficient flux from bipolar regions at lower latitudes has reached the pole.

Homann et al. (1997) found recently in PFs an unexpected strong magnetic fields of $B=1600 \pm 350 \mathrm{G}$ which are very unusual for structures outside active regions at high latitudes of the Sun. According to Homann et al. the magnetic polarity of the PFs is that of the general polar magnetic field, while Zhang \& Zhang (1999) find no preference of polarity of small-scale magnetic structures at the solar poles. 


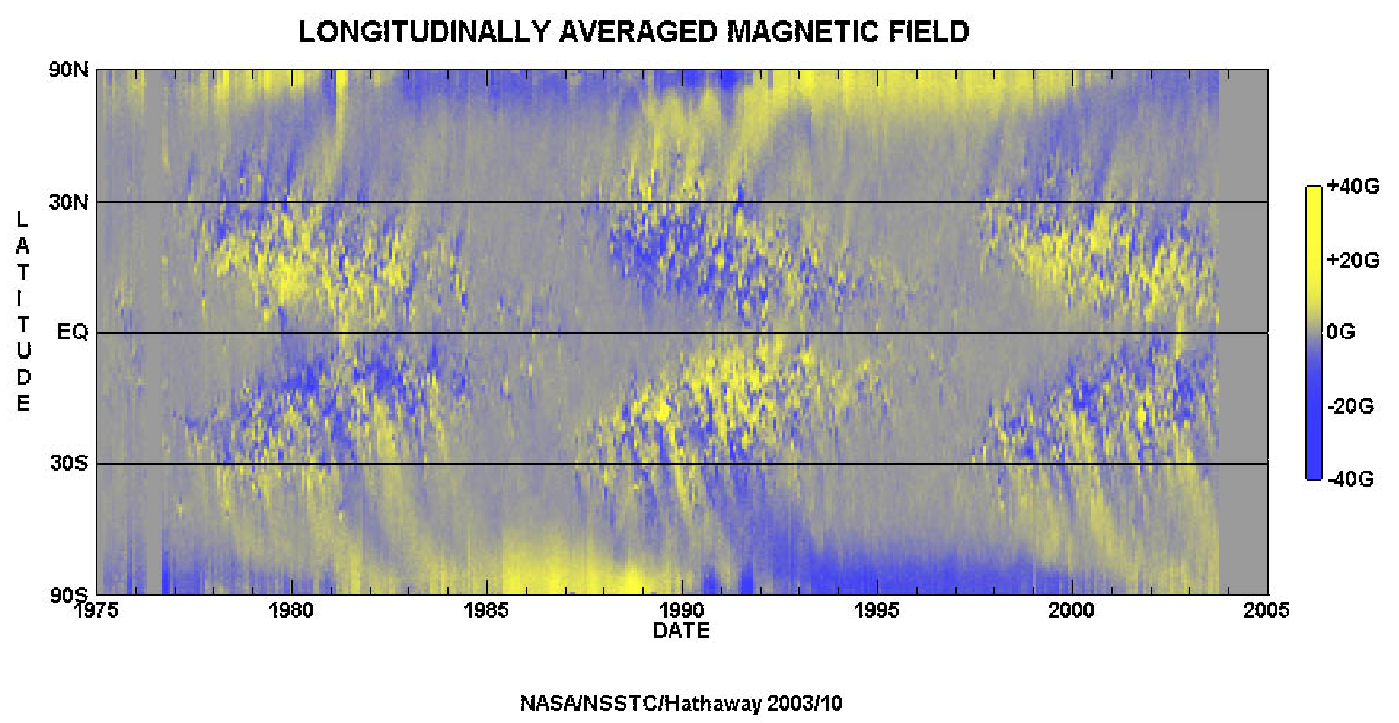

Figure 1.1: Butterfly diagram of solar magnetism

As mentioned above polar faculae are usually observed in white light and spectral lines in the visible spectral range and in the near ultraviolet. Observation of PFs in other wavelengths is difficult because of the absorption in the terrestrial atmosphere and of the requirements of high spatial resolution for studying such small-scale structures. However, the solar activity in general and PFs in particular can be studied by means of observations in radio microwave frequencies, for which the earths atmosphere is transparent. Riehokainen et al. (1998) have performed and analyzed radio-observations at the frequency of $36.8 \mathrm{GHz}$. The distribution and time variation of the so called enhanced temperature regions (ETRs) in the polar zones of the Sun were studied and it was found that ETRs can be related to polar faculae. Earlier studies also reveal the connection of ETRs in radio wavelengths to PFs. It was shown by Babin et al. (1976) and Efanov et al. (1980) that ETRs coincide with the regions of magnetic field intensifications. Makarov at al. (1991) have found from the observations with RATAN 600 at $\lambda=2.3 \mathrm{~cm}$ that ETRs could be connected to polar faculae observed in optical wavelengths.

Observations of polar activity in other wavelengths can be performed only from the space. In the recent studies Riehokainen et al. (2001) have compared ground based whitelight and radio observations with $171 \AA$ EUV SOHO/EIT images. It was found that ETRs in general coincide with the dark areas seen in SOHO/EIT images, and bright structures in SOHO/EIT images encircle groups of polar faculae.

DeForest et al. (1997) suggest from coordinated observations with the EIT and MDI instruments on board the SOHO spacecraft that polar coronal plumes are rooted in X-ray bright points corresponding to unipolar magnetic concentrations on network cell boundaries. Yet other authors, e.g. Wilhelm et al. (1998), argue that polar plumes have their footing in closed loop structures, and lateron Wilhelm et al. (2000) identify the outflow regions as dark coronal areas, not as the bright plumes. Thus, the research into the magnetic cycle at the solar poles, the origin of the fast solar wind, and the rôle of the polar coronal plumes makes the study of PFs an important issue. 
Apart from the notion of strong fields by Homann et al. (1997) little is known about the internal structure of PFs. Hence, the present study aims at scrutinizing their spatial fine structure, including the fine structure of their magnetic fields, and the temporal evolution of PFs on short time scales. White light and spectropolarimetric data have been analysed with emphasis on high spatial resolution and high polarimetric sensitivity at the same time. Both are needed for a study of structure and dynamics of PFs since these are smallscale features and since the polarimetric signals from small structures at the poles of the Sun, i.e. close to the solar limb, are small. The results of the data analysis allow to test magnetic flux tube (MFT) models and their compatibility with observations close to the solar limb.

Thus, the goal of the present work is to study the structure of PFs in detail and to verify the hypotheses about PFs as concentrations of small-scale magnetic flux tubes by means of high-quality observations and a theoretical study:

a) analyses and interpretation of high-resolution spectroscopic, polarimetric and whitelight observations - investigating the physical properties of PFs: size, intensity distribution, magnetic field strength, velocities and dynamic evolution. The observations, the data reduction and analyses and the observational properties of PFs will be presented in Chapters 2 and 3 of this thesis;

b) numerical modeling and radiative transfer calculations with oblique rays passing through an inhomogeneous magnetic medium. The methods of modeling are described in Chapter 4, and the modeling results are compared with the observations in Chapter 5. 



\section{Observations and Data Reduction}

The current chapter of the thesis deals with the observational study of polar faculae. PFs belong to the class of small-scale magnetic structures of the solar photosphere, therefore observations of such structures require high spatial and spectral resolution, and high spectropolarimetric sensitivity. Polar faculae as well as equatorial faculae can be observed only near the solar limb, where they have increased brightness contrast. Observations at high heliocentric angles have their own peculiarities. Close to the limb we see higher layers in the solar photosphere than for observations at the disk center. This is due to the fact that at the limb the geometrical path of the ray through the solar atmosphere increases, resulting in more absorbers along the ray, and a bigger optical thickness. For a planeparallel atmosphere we have $\tau_{\mu}(z)=\tau_{\mu=1.0}(z) / \mu$, where $\tau(z)$ stands for optical thickness of the plasma between the observer and a certain height $z$ in the solar photosphere, and $\mu=\cos (\theta)$ is the cosine of the heliocentric angle. In other words, if observing in white light at the center of the solar disk we see, roughly speaking, the layer at $\tau=1$, usually taken as a reference height for atmospheric models $(z=0 \mathrm{~km})$. Near the limb $(\mu=0.1$ for example) $\tau=1$ will correspond to a geometrical height $z$ where $\tau_{\mu=1.0}=0.1$, which is approximately $160 \mathrm{~km}$ higher. The upper layers, due to the negative temperature gradient in the photosphere, are cooler, therefore the emergent intensity is lower, producing the so called limb darkening. According to Allen (1976) $I_{\lambda}(\mu=0.2) / I_{\lambda}(1)=0.43$ for $\lambda=5000 \AA$ and at the extreme limb $I_{\lambda}(\mu=0.1) / I_{\lambda}(1)=0.31$. For the observer it means that observations near the limb are characterized by lower counts and poorer SNR.

It is generally adopted that unresolved solar magnetic fields consist of concentrations of isolated small-scale magnetic flux tubes, characterized by a tiny spatial size and strong fields (e.g. Beckers \& Schröter 1968; Stenflo 1973, 1994). Due to the buoyancy force, magnetic flux tubes are mainly vertical, at least in the height range in the photosphere from where the considered spectral lines originate. Polarimetric observations obtained in the frame of the current work were limited by measuring, apart of the intensity $I$, only the Stokes $V$ component, describing circular polarization of the radiation, and sensitive only to the line-of-sight component of the magnetic field $B$. The Stokes $V$ signal measured at a certain heliocentric angle $\theta$ will be reduced by the factor of $\mu=\cos (\theta)$ in comparison with the observation of magnetic fields of the same magnitude but at the center of the solar disk. Also, due to the negative gradient of $B$ we expect weaker magnetic signals from higher layers observed at the limb.

Thus, for the reasons mentioned above (low counts at the limb, weak polarimetric signal from inclined unresolved fields, and small geometrical size of the structures) we can conclude that for a successful study of limb faculae spectropolarimetric observations with the highest possible quality and sensitivity and obtained under best seeing conditions 
and with high spatial and spectral resolution are necessary. Of course, problems of solar polarimetry are not limited to the study of polar faculae. Observations and understanding of all types of small-scale magnetic fields pose very high requirements on the quality of observations. Limb faculae in general and polar faculae in particular, in addition to traditional problems of observing small-scale magnetic fields, have limb effects.

Nowadays observations satisfying all the mentioned requirements together still remain inaccessible. One characteristic of the observations can be improved at the cost of deteriorating another one. For example, data with high signal-to-noise ratio can be obtained by increasing the exposure time, which in turn leads to the decrease of spatial resolution, and vice versa. In addition all ground-based observations exhibit the degrading influence of the earth's atmosphere.

The solution is in building bigger ground-based solar telescopes such as GREGOR, a $1.5 \mathrm{~m}$ telescope for solar research (e.g. von der Lühe et al. 2001), able to collect more photons in order to increase the spectropolarimetric sensitivity, to use systems of adaptive optics for stabilization of the image "on the fly" and to develop and apply image restoration algorithms for post factum image reduction to correct for seeing effects. Another branch of development is to avoid seeing by bringing solar telescopes beyond the earth atmosphere to the stratosphere (e.g. Krat et al. 1972; Mehltretter 1976; SUNRISE project: Schmidt et al. 2001) or to the space.

The observations which form the basis of this study were performed with the German solar telescopes: Gregory-Coudé Telescope - GCT, and Vacuum Tower Telescope VTT at the Observatorio del Teide on Tenerife. The altitude of the observatory is $2400 \mathrm{~m}$ above sea level. The observations with the GCT and its Czerny-Turner spectrograph are characterized by high spectral resolution and high spectropolarimetric sensitivity, which are typical for slit spectrographs, on one side, and moderate spatial resolution, limited by the slit width, exposure time and seeing conditions on the other side. For the observations with the VTT a scanning Fabry-Perot interferometer ("Göttingen" FPI) was used as a postfocus instrument. This instrument allows simultaneous observations of true two-dimensional (2D) spectra and of white-light images. A sequence of short exposure broadband images (so called speckle images) is used for further "speckle reconstruction", aiming at correcting for seeing effects and to achieve diffraction limited images (de Boer 1993, 1996). The instant optical transfer function found from reconstructed broadband images is used afterwards for correcting the narrowband filtergrams, obtained with FPI etalons, for atmospheric distortions (e.g., Keller \& von der Lühe 1992, Krieg et al. 1999 and Koschinsky et al. 2001). In such a way the VTT observations can be reduced to data free of atmospheric influence and with high spatial resolution.

During one of the observational campaigns we made an attempt to observe simultaneously the same area in the solar photosphere with both telescopes to use the advantages of both telescopes, high spectral resolution of a slit spectrograph and high spatial resolution of reconstructed VTT-FPI data. The main problem was to point two telescopes to the same area on the solar disk, using as a reference very faint and sometimes hardly detectable objects as isolated faculae near the limb. As a result, we did not succeed to obtain high quality data with good seeing from simultaneous observations with the two telescopes. So, in the current work, we restrict our study to only independent observations with both telescopes, obtained at different times. 
In the following sections the observations with both telescopes will be described in more details. Descriptions of data reduction, analysis and possible interpretation of the spectropolarimetric observations will be presented. Finally, the observational properties of the phenomena under consideration will be summarized, which will serve as constraints for numerical modeling.

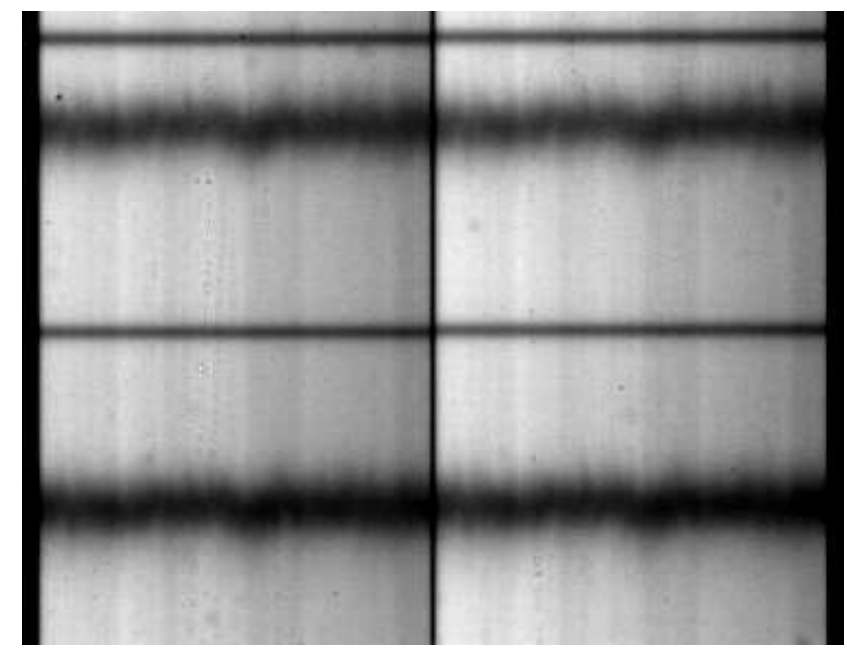

Figure 2.1: Example of raw image observed with the GCT, one-dimensional spectra at $6302 \AA$ separated into left and right circularly polarized beams. Wavelength increases from bottom to top. The two broad lines are solar Fe I lines under study, the two narrow lines are telluric $\mathrm{O}_{2}$ lines.

\subsection{One-dimensional spectrograms observed with Gregory-Coudé Telescope}

The Gregory-Coudé Telescope (GCT) was an evacuated solar telescope with a prime mirror of $45 \mathrm{~cm}$ diameter and $250 \mathrm{~cm}$ focal length. An elliptical secondary mirror (Gregory mirror) enlarges the image by a factor of 10 , resulting in a diameter of the solar image of $25 \mathrm{~cm}$, of which only one-tenth is selected. The coudé mounting (in contrast to a coelostat system) provides a low and constant instrumental polarization. The main post-focus instrument is a $f=10 \mathrm{~m}$ horizontal Czerny-Turner spectrograph, with the slit in the coudé focus. The resolving power of the spectrograph is $\lambda / \Delta \lambda \sim 500000$. A slit-jaw imaging in three channels (white light, $\mathrm{H} \alpha$ and $\mathrm{Ca} \mathrm{K}$ ) was available. The GCT was operated by the Universitäts-Sternwarte Göttingen at the Observatorio del Teide/Tenerife since 1985, and it was dismounted in Mai 2002 to be replaced by a new $1.5 \mathrm{~m}$ GREGOR telescope. For the present study, observations were used which were obtained during one of the last observational campaigns at GCT.

The observations for this work were obtained on 8 June, 2001 and 9 August, 2001. Several areas in the vicinity of the south and north poles and at high heliocentric angles $(\mu<0.3)$ containing polar facular points were observed. Also a few regions with network equatorial faculae at low latitudes were recorded. The identification of facular points and 
positioning of the image on the spectrograph slit was made with the help of slit-jaw images in white light in the case of polar faculae. For weaker network faculae $\mathrm{Ca} \mathrm{K}$ images, in which magnetic elements exhibit increased contrast, were used.

Spectrograms were obtained in two magnetically sensitive iron lines: the normal Zeeman triplet Fe I $6302.5 \AA$ (Landé factor $\mathrm{g}=2.5$ ) and the transition belonging to the same

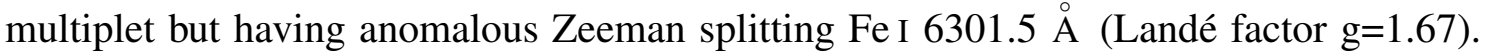
The characteristics of the observed lines are given in Table 2.1. Both spectral lines are formed in photospheric layers and are well suited for studies of magnetic structures in lower atmospheric layers. The distance between the lines is about $1 \AA$, what allows simultaneous recording of spectra in both lines.

Table 2.1: Characteristics of observed spectral lines

\begin{tabular}{ccccccc}
$\lambda, \AA$ & $E_{p}, \mathrm{eV}$ & Transition & $\lg (\mathrm{gf})$ & EqWidth, $\mathrm{m \AA}$ & $\mathrm{H}, \mathrm{km}$ & $g_{\mathrm{eff}}$ \\
\hline 6301.5 & 3.65 & $z^{5} D-e^{5} D$ & -0.59 & 127 & $\sim 500$ & 1.67 \\
6302.5 & 3.68 & $z^{5} D-e^{5} D$ & -1.16 & 83 & $\sim 400$ & 2.5
\end{tabular}

The Stokes $V$ polarimeter, consisting of a $\lambda / 4$ retarder plate and two crossed calcites, was mounted behind the slit. It splits the beam into right and left circularly polarized light (Fig. 2.1): $\frac{1}{2}(I+V)$ and $\frac{1}{2}(I-V)$. The microscanner (Stolpe \& Kneer 1998) was mounted in front of the slit, it was used to move the image across the slit and thus to obtain spectrograms from different spatial positions. Slit-jaw images in white light, in $\mathrm{H} \alpha$ and in $\mathrm{Ca} \mathrm{K}$ were taken simultaneously with the spectrograms.

We summarize the specifications and the advantages of observations with GCT:

- Gregory-Coudé system with low and slowly varying instrumental polarization,

- high spectral resolution $\lambda / \Delta \lambda \sim 500000$, spectral sampling $\Delta \lambda=5.76 \mathrm{m \AA}$,

- higher signal-to-noise ratio (SNR) than from two-dimensional (2D) spectrometer (see Sect 2.2 below),

- simultaneous recording of bigger spectral range $(\sim 2 \AA)$ than with 2D spectrometer with good sampling,

- CCD camera La Vision with size of $384 \times 286$ pixel, sampling in spatial direction $\Delta x=0.19$ (corresponding to the pixel distance of the CCD detector),

- exposure time $0.2 \mathrm{~s}$ and $0.3 \mathrm{~s}$,

- quasi 2-dimensional spectra: scanner mounted in front of the slit moves solar image across the slit with step width of $81 \mu,(0.68)$. The typical number of steps of the scanner was 40 (optionally 60),

- Stokes $V$ polarimeter,

- flat fields, dark offset, spectrograms with 'tree pattern', (a regular transmission pattern to be placed in front of the spectrograph slit) were also recorded for further reduction and calibration of the data, 
- observed data: 3D data cube $I(x, \lambda, y)$ with size $\sim\left(28^{\prime \prime}, 2 \AA, 27^{\prime \prime} .2\right)$, sampling $\left(0^{\prime \prime} .19\right.$, $\left.5.77 \mathrm{m \AA}, 0^{\prime \prime} 68\right)$ and resolution $\left(1^{\prime \prime}\right.$, high, $\left.1^{\prime \prime}\right)$, respectively.

The disadvantages of the GCT observations are typical for slit spectrographs: a) high SNR is provided at the expense of long exposure time of $300 \mathrm{~ms}$ and large slit width $\sim 120 \mu=1^{\prime \prime}$; b) moderate spatial resolution (not better than $1^{\prime \prime}$ ) limited by the slit width and seeing conditions; c) only real 1D spectra are observed, the use of a scanning device allows to obtain quasi 2D images, but the spatial resolution is usually lower in the direction perpendicular to the slit than along the slit.

\subsubsection{Standard data reduction}

The raw data obtained during the observations need to be reduced before we can start the analysis of the observations. To correct the data for the dark offset and the flat fields the following, well known expression was used

$$
\text { Reduced Data }=\frac{\text { Raw Data }- \text { Dark Frame }}{\text { Flat Field }- \text { Dark Frame }}
$$

In spite of the apparent simplicity of the formula, the procedure of the reduction and calibration of 1D spectropolarimetric data has some peculiarities which will be explained in the following section.

\section{Dark frame}

The correction for dark events is an important part of data reduction. According to the principles of the photoeffect the voltage counts from a pixel (proportional to the number of electrons knocked out by photons in the cell of the CCD chip) should be strictly proportional to the intensity of light. CDD chips have a good linearity in the operating range of intensities. What should not be forgotten is the dark offset. Even when the CCD is not illuminated there are always some thermally induced charges on the CCD. Each pixel has its own dark voltage, so we take a dark frame. Apart of intrinsic dark voltages there is also unavoidable noise (read noise and thermal noise). To reduce this noise and to improve the quality of the reduction it is advisable to take several dark frames and average them.

During the observations, dark files were taken with closed spectrograph shutter and under the same conditions as the other files: with the same exposure time and with cooling of the CCD chip which kept its temperature at a constant level. Also each file contains the same number of frames (40 or 60), which were averaged to get one pure dark frame with low noise. Another way to improve the dark correction is to fit the average dark frame to a smooth surface described by an analytical function. The distribution of the dark voltage on the chip depends on the structure of the crystal. In the used chip we find an approximately constant gradient of counts from one side to the other, which can be well fitted by a first order surface (plane), completely free of noise. Since this total dark voltage is additive, we can remove it by subtracting the dark pixel values from the exposure pixel values both from the data files and from the flat fields. 


\section{Flat fielding}

Flat field effects can also be calibrated out. They are different from dark frame effects in that they are multiplicative. Some pixels are more efficient than others and will build up signal at a faster rate. To calibrate this we take an exposure of an object we suppose to be flat, has no variations in brightness. Flat fields were obtained in the same spectral range changing, during exposure, the telescope pointing by manual control and trying to avoid active regions. To improve the reduction an average of many flat fields was used.

The average flat field frames still contain spectral lines. To remove these the image containing the flat field was first averaged in spatial direction, resulting in an averaged one-dimensional spectrum. It was then expanded back along the spatial direction such that the resulting image contains only spectral lines. Dividing now the flat field by this created artificial spectrum should remove the spectral lines and form the gain table. Yet a problem arose because the spectral lines are not strictly parallel to one side of the CCD chip. Comparing the positions of spectral line centers at both ends of the slit from the averaged frame (free of Doppler shifts), the relative angle was found to be $0.13^{\circ}$. Typically one frame has a size of 150 and 280 pixels in dispersion and spatial directions, respectively. So, introduced by a rotation of the CCD with respect to dispersion and spectral lines, offsets in horizontal and vertical directions can be estimated as $\delta \lambda \sim 0.25$ pixels $=$ $1.5 \mathrm{~m} \AA$ and $\delta x \sim 0.47$ pixels $=00^{\prime \prime} 09$

Practically, for the procedure of flat fielding, the averaged artificial spectrum is slightly broadened, and the division of the flat field frame by it will introduce artifacts. To improve the reduction we have proceeded in the following way. Before averaging along the slit, each single column (perpendicular to dispersion) was corrected for the offset introduced by the rotation, it was shifted by a corresponding fractional amount of pixels. The average unbroadened spectrum was expanded back to produce a two-dimensional frame and each column was shifted back. Dividing now the flat field by the latter will produce the desired gain table without any sign of spectral line.

\section{Correction of geometrical distortions}

The next step after dark voltage correction and flat fielding is a geometrical correction. Unfortunately, the observed spectra exhibit several geometrical distortions which should also be corrected. Apart from the inclination of spectral lines, described above, due to the rotation of the CCD chip with respect to the slit, there are also distortions produced by the different paths of the polarized beams through the spectrograph. It means in praxis that the distances between two points on the solar image on the detector differ slightly from each other in the right and left beams. The same happens in wavelength direction. The left and right beams are stretched and squeezed with respect to each other. The tree pattern images are used for the correction in spatial direction. For correction in wavelength direction averaged iron lines and the telluric spectral lines (cf. Fig. 2.1) were used as references. To correct for the rotation we proceeded in the same way as with flat field reduction, shifting each column by an appropriate fractional offset, known from the flat fields. 


\section{Correction for infrared background and spectroscopic stray light}

To evaluate the spectral quality of the observed data, the averaged flat field spectrum is compared with the corresponding spectral range taken from the high resolution Fourier Transform Spectrometer (FTS) atlas by Brault \& Neckel (1987), quoted by Neckel (1999). For averaging, flat field frames observed near disk center were selected (to avoid Doppler broadening due to the rotation of the Sun) and corrected for dark voltage. The thus obtained average line profiles and the ones from the atlas were normalized to a common continuum. It was found that all recorded spectral lines, two photospheric iron lines and two telluric lines, are weaker (have smaller depression) than the reference line profiles.

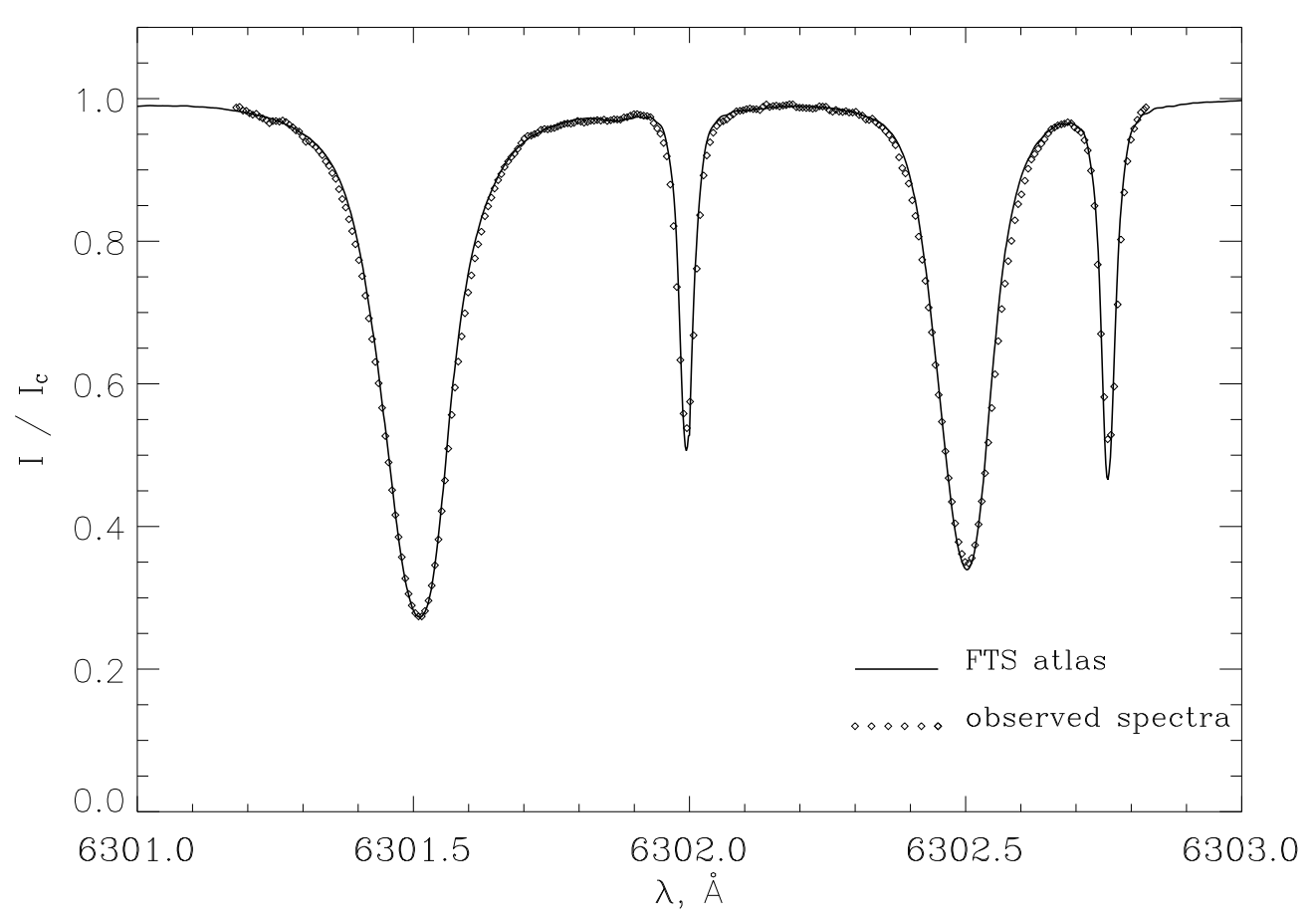

Figure 2.2: Observed spectral range (dotted curve) corrected for infrared contamination and spectrographic stray light in comparison with reference spectra (solid curve) taken from FTS atlas (Brault and Neckel 1987, quoted by Neckel 1999). The distance between the dots $\Delta \lambda=5.8 \mathrm{m \AA}$ corresponds to the spectral sampling of the data.

The possible explanation of such a discrepancy is in an infrared contamination. The narrowband (FWHM $\sim 10 \AA$ ) interference filter (central wavelength $\lambda=6302 \AA$ ) mounted behind the polarimeter and used for the selection of the order of the spectrograph grating probably has had no infrared blocking. CCD cameras exhibit strong response at nearinfrared wavelengths from 7000 to beyond $11000 \AA$. Stray light in the spectrograph, mainly from the grating and subsequent optical surfaces, causes a "white light" background. It results in parasitic counts on the CCD chip, creating an additional offset, similar to dark voltage. The subtraction of the dark frame is obviously not sufficient because darks are exposed with closed shutter, when no infrared photons can reach the CCD. 
It was found empirically that a subtraction of $10 \%$ of the continuum intensity from the spectrograms leads to a good agreement of the observed line profiles with the ones from the FTS atlas (Fig. 2.2).

The importance of this correction should not be underestimated. An additional constant offset in the counts reduces the observed line depression, which in turn leads to an underestimation of Stokes $V$ amplitudes.

\subsubsection{Noise filtering}

Noise filtering is a very important part of data reduction. For the analysis of polarimetric observations of solar small-scale magnetic fields, which are characterized by weak amplitudes of the signal, noise filtering is especially important. It will become even critical if the data under consideration were obtained near the limb where, as it was already mentioned, SNR is at least two times worse than for structures observed at the center of the solar disk.

Noise is an unavoidable signal component inherent to every electronic imaging system. There exists a variety of sources of noise. For CCD imaging systems the main contribution to the noise comes from the imperfectness of the electronics of the camera system (so called read noise), which can be reduced through precision electronics, and statistical variation of the number of photoelectrons collected by a CCD pixel (photon noise). Photon noise is a fundamental property of the quantum nature of light, and it cannot be reduced via camera design and should be filtered afterwards.

In this section we will discuss two approaches to noise filtering of 1D spectrograms: by means of Fourier analysis and of singular value decomposition.

\section{Lowpass frequency filter}

A common and widely used way of filtering is based on a Fourier analysis. It consists of the following subsequent steps: transformation of the original image into the spatial frequencies (wavenumbers $k$ ) domain by means of Fast Fourier transformation (referred to as FFT), estimating of a cutoff frequency $f_{0}$ and construction of a lowpass frequency filter, multiplication of the Fourier image with the latter and transformation back to the original coordinates:

$$
S_{f}=\left\{S^{F F T} \cdot \text { Filter }\right\}^{-F F T}
$$

where $S$ is the original unfiltered signal, ()$^{F F T}$ and ()$^{-F F T}$ denote direct and inverse Fourier transformation. The resulting filtered image $S_{f}$ will be smoothed and will contain no information on spatial periods $x<1 / f_{0}$. It is a robust and efficient method. The only items which are of some concern are the estimation of the cutoff and the shape of the filter. The choice of the cutoff spatial period can be made from the a priory knowledge of the factors which were limiting the spatial resolution during the observations. The lowest bound is set by the diffraction limit of the telescope and twice the sampling, which for the GCT and the used detector are 0.35 and $0{ }^{\prime \prime} 38$, respectively. A higher limitation on the resolution is set by the width of the spectrograph slit ( $1^{\prime \prime}$ and $0^{\prime \prime} .75$ for our data). And finally, seeing conditions diminish the resolution of the observations typically to $1^{\prime \prime}$. A more sophisticated approach to the choice of $f_{0}$ is based on the analysis of the data itself. 
It does not require any a priory knowledge about the conditions under which the data were taken. Applying FFT to the data and plotting the power spectrum (Fig. 2.4, gray solid curve), the cutoff can be chosen at the point where the power spectrum becomes flat, i.e. where the power becomes independent of frequency. One assumes that this flat part contains no signal. Another possibility is to find the power spectrum of the noise from the flat fields, supposing that the flat fields contain nothing but noise.

Now, when the cutoff frequency is defined, the next question is which shape of the filter to use. The most simple filter is a step function, equal to 1 for $f<f_{0}$ and equal to 0 for $f>f_{0}$. Instead of a step function, a smooth function with fast-falling apodisation wing can be used. A common type of such filter in the frequency domain is a Butterworth frequency filter. The general form of a low-pass Butterworth frequency filter is given by

$$
H=\frac{1}{1+{\frac{f}{f_{0}}}^{2 n}} .
$$

Here $f$ is the spatial frequency, $f_{0}$ is the nominal filter cutoff frequency, and $n$ is the order of the filter.

A more advanced filter which considers the character of the signal and of the noise was constructed by Brault and White (1971) and is called the optimum filter.

$$
\tilde{\Phi}(s)=\frac{P_{S}(s)}{P_{S}(s)+P_{N}(s)} .
$$

Here $P_{S}(s)$ is the power spectrum of the signal alone, $P_{N}(s)$ the power spectrum of the noise, and total power $P_{S}(s)+P_{N}(s)$ is a good approximation to the power of the observed signal. The problem is that in order to construct such a filter one should know $P_{S}(s)$ - the power spectrum of the desired pure signal without noise. Usually $P_{S}(s)$ is replaced by the model of the power spectrum of the signal. The choice of the model depends on the nature of the signal. In our case it is a variation of the intensity along the slit of the spectrograph and the shape of its power spectrum in logarithmic scale reminds on a dispersion profile with a pronounced core and extended wings, so it can well be fitted by the combination of Gaussian and Lorentz functions. In such a way the optimum filter can be constructed and applied to the signal in Fourier domain.

Finally, the choice of the filter depends on the data. For our observations it was found empirically, that the use of a Butterworth frequency filter gives satisfactory results and it can be much easier realized than the optimum filter. Among the disadvantages of the filtering in Fourier domain we mention:

- one-dimensionality of the Fourier transformation: Fourier analysis is based on the series expansion of the signal with sin and cos as basic functions. Harmonic functions are one-dimensional by definition, therefore Fourier transformation is defined only for $1 \mathrm{D}$ signals, and when applying to the $2 \mathrm{D}$ image or spectrogram it is applied to rows and columns independently and consequently. For filtering in Fourier domain one has to estimate the cutoff frequency and to construct the filter for both dimensions independently.

- non-locality of the analysis: The non-local properties of the Fourier transformation result from the non-locality of the basic functions. It means inability of the 
method to remove noise locally. Instead of it the filter will take away the whole harmonic of a given frequency.

- compromise between degree of filtering and achieved spatial resolution: noise can be efficiently removed only at the expense of degradation of the resolution.

\section{Application of Singular Value Decomposition for noise filtering}

As an alternative to noise filtering in Fourier space a Singular Value Decomposition (SVD) can be used (see e.g., Joliffe 1986). For the purpose of noise filtering the idea of the SVD method can be expressed by the formula

$$
A=\sum_{i=1}^{r} a_{i}
$$

Every real matrix $A$ (image) can be represented as a sum of $r$ images $a_{i}$ of the same size, where $r$ is the rank of the matrix. In practice, for images, $r$ is equal to the smallest dimension of the image. Each component of the decomposition can be written as $a_{i}=\sigma_{i} u_{i} v_{i}^{T}$, where $\sigma_{i}$ is a scalar singular value, $u_{i}$ and $v_{i}$ are vectors. More details about SVD and its apllication to noise filtering and image procesing can be found in Martin Gander (http://www.math.mcgill.ca/mgander/consulting/northpoint/SVDFilter.ps.gz). The components of SVD are ordered in descending sequence of corresponding singular values, or in accordance with their "significance" or contribution of each component to the original image in such a way that $a_{1}$ has the highest singular value, carries the strongest power, and has the biggest contribution to the original image. Adding more components will refine the result $a_{1}+a_{2}+\ldots$, more details will appear. It is the opposite with the very last terms in the summation, they have smallest singular values, carry very low power, and the image will not suffer from neglecting them. Thus, taking only the first $N_{0}$ terms in the summation, and removing the rest, we can filter the image from the noise.

$$
A=\sum_{i=1}^{N_{0}} a_{i}+\sum_{i=N_{0}+1}^{r} a_{i}=\text { Signal }+ \text { Noise }
$$

The question is when to stop the summation? Which $N_{0}$ to take? This method of noise filtering reminds filtering in Fourier domain. In both cases the signal is decomposed (expanded in case of FFT) into a sum, only the first terms are taken, and the rest is neglected. The principal difference is that Fourier analysis deals with harmonic functions and frequencies and SVD with singular values. Therefore the analogy with cutoff frequency will not work for SVD and another criterion has to be found.

To find such a criterion we have proceeded in the following way. Changing the number $N_{0}$ of "cutoff singular value" from 2 to $r$ we examined the residual part of the decomposition $R_{N_{0}}=a_{N_{0}+1}+a_{N_{0}+2}+\ldots+a_{r}$. The purpose was to find $N_{0}$ such that the residuum will contain no information, but pure noise. Noise is usually characterized by the weak dependence on spatial frequency, in ideal case noise is white. So for each $N_{0}$ the power spectrum of $R_{N_{0}}$ was calculated and its dependence on frequency was examined. Empirically it was found that the choice of $N_{0}$ between 10 and 15 results in an efficient noise filtering of spectrograms without any loss of information as demonstrated in Fig. 2.3. 


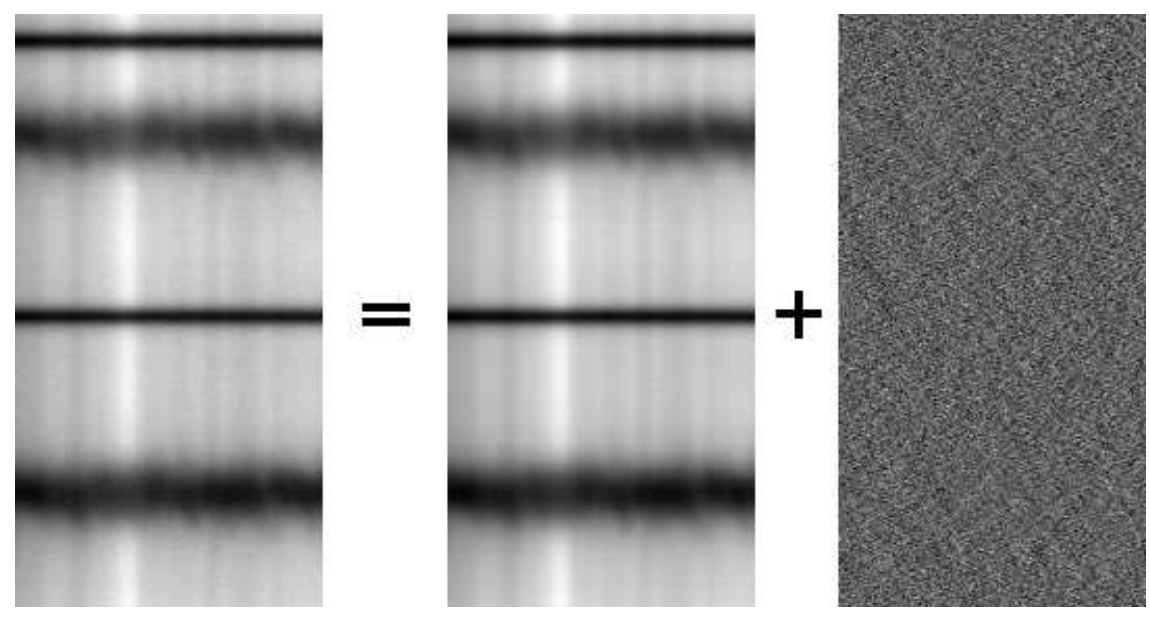

Figure 2.3: Illustration of the filtering of Stokes $I$ spectrograms in singular value domain. By means of SVD the original image is decomposed into the sum of two frames. The filtered one contains the first 10 components of the SVD. The residual image is considered to be the noise.

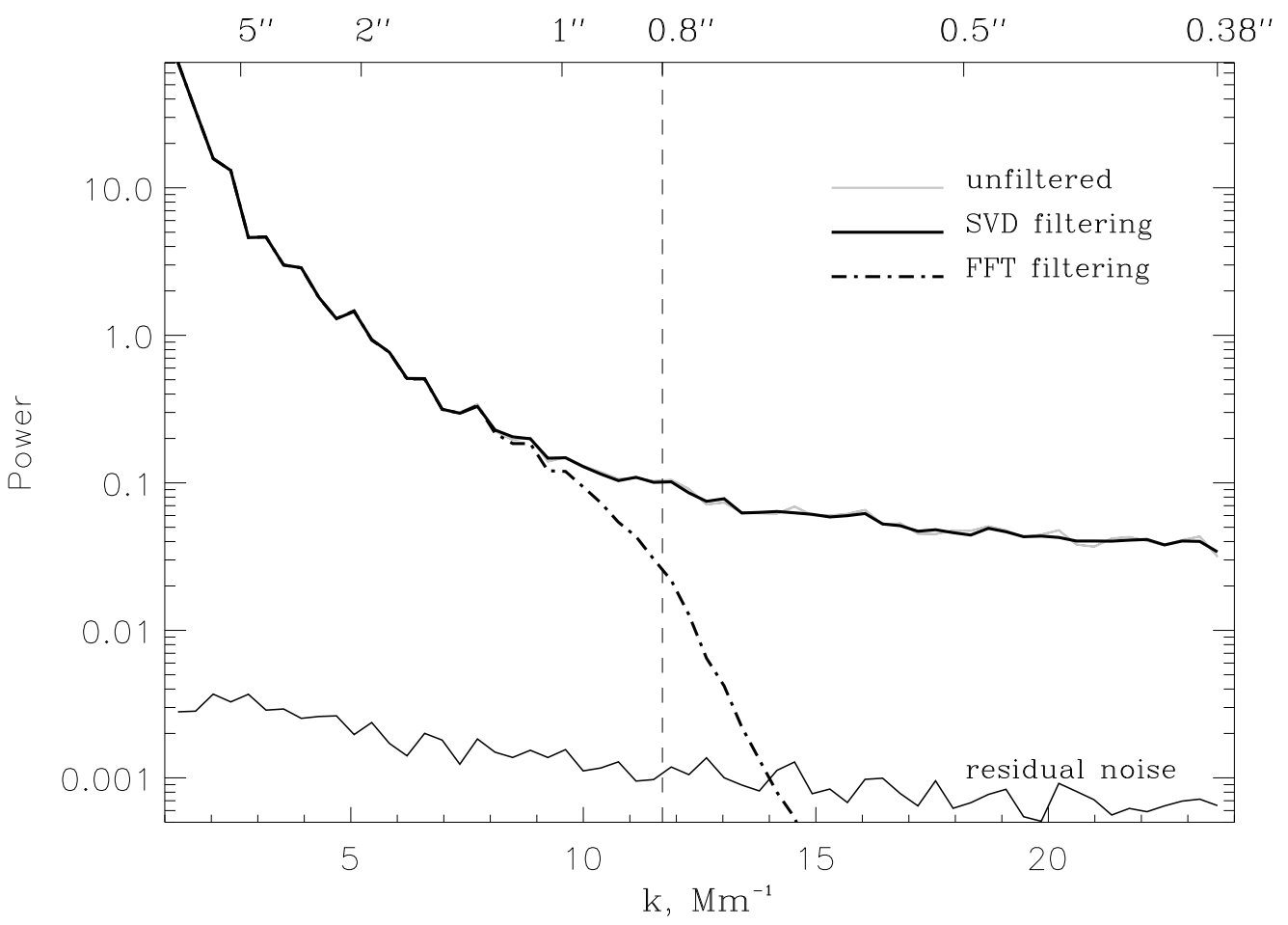

Figure 2.4: The power spectra of the unfiltered image and filtered with two methods: lowpass Butterworth frequency filtering in Fourier domain, and SVD filtering. The vertical line corresponds to the cutoff frequency used for the lowpass filter. The lower curve is a power spectrum of the residual noise, which was filtered out by means of SVD. 
Filtering in singular value domain has an interesting property. It does not cut off frequencies in contrast to Fourier filtering (Fig. 2.4). The power spectrum of the unfiltered signal and of filtered by means of SVD look practically identical. Noise can be essentially removed without affecting the spatial resolution of the data. This very valuable property of the method is the direct consequence of the local character of the SVD analysis. Another peculiarity of SVD is that it is a true 2D analysis. Application of SVD to the reduction of 1D spectrograms appears to be very natural because typical images under consideration consist of structures which can be classified in terms of columns (spectral lines) and rows (intensity profiles along the slit).

\subsubsection{Reduction of Stokes $V$ spectrograms}

The steps of data reduction described so far were applied to Stokes $I$ spectrograms or to left and right circular polarized beams separately. However, Stokes $V$ is obtained by subtracting two beams from each other, and in the resulting differential images the next order distortions start to play a role. Therefore, polarimetric signals require additional treatment. Observed Stokes $V$ spectrograms contain random noise and systematic distortions apart from useful signal

$$
V_{\mathrm{obs}}=V_{\text {true }}+\text { Systematic Distortions }+ \text { Random Noise }
$$

The noise can be filtered to some extent applying one of the above filters, for example keeping only the first 10 components of the SVD. But the resulting image (Fig. 2.5) will still contain systematic distortions and a relatively high level of noise, limiting the polarimetric sensitivity of the data. The systematic part can originate from the imperfection of the flat fielding procedure, namely that structures of the CCD chip, dust and interference fringes were not completely removed by flat fielding. All these artifacts can be neglected in Stokes $I$ spectra, but Stokes $V$ is characterized by a hundred times weaker signal.
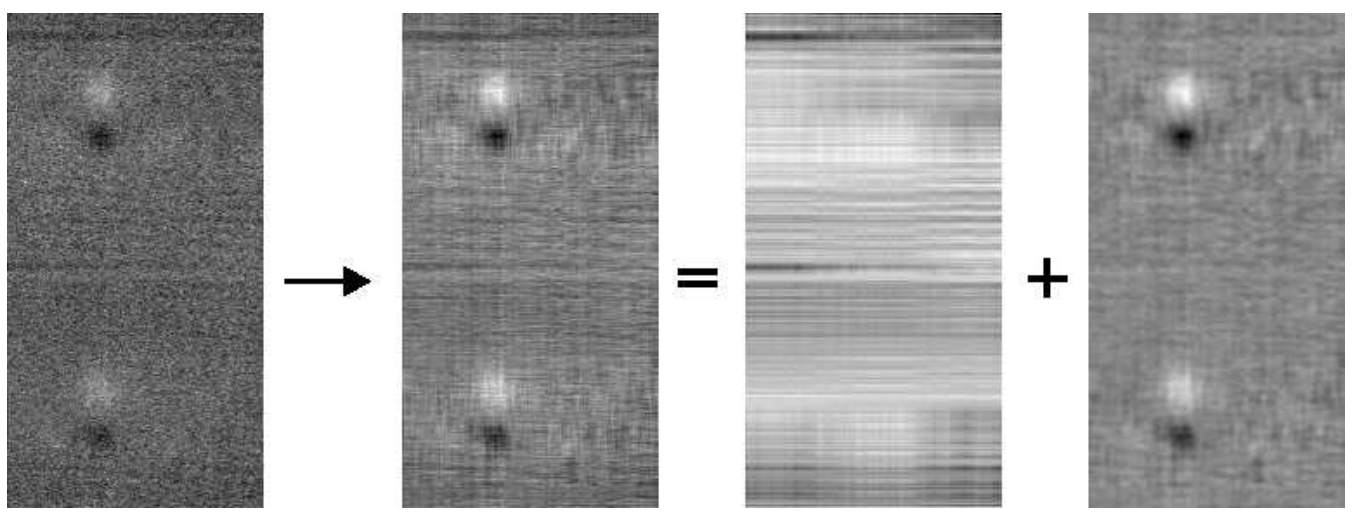

Figure 2.5: Reduction of Stokes $V$ spectrograms by means of SVD. From left to right: 1) spectrogram after standard reduction before noise filtering; 2) noise filtering in singular value domain $\left(N_{0}=10\right)$. From the latter systematic artifacts (3) are subtracted. The last (4) in the row is the resulting reduced spectrogram. 
The same holds for the geometrical distortions caused by different paths of left and right beams. The procedure described above gives satisfactory results for Stokes $I$, but not sufficient in the case of $V$ signals. If the left and right beams are stretched and squeezed even very little in wavelength direction with respect to each other, the subtraction will produce systematic artificial Stokes $V$.

To separate the second term in Eq. 2.4, describing constant distortions, from the residual noise and $V$ signal we have averaged frames which contain 1D Stokes $V$ spectra corresponding to different positions of the slit, excluding frames containing evident magnetic signal, frames when the slit of spectrograph was above faculae. Such averaging diminishes the level of noise, while the systematic error remains at a constant level. The result in this form can already be used for correction, but it still contains noise. To improve the reduction and to get the pure correction frame we have applied a singular value decomposition to the averaged frame and neglected all components except the first three. This frame contains only the systematic part and is subtracted from the spectrograms. The resulting reduced frame (Fig. 2.5 right frame) is free of all kind of distortions and contains only the signal and remaining weak noise.

\subsubsection{Intensity maps}

After various corrections and calibrations, which were described in this chapter, the reduced 1D spectrograms can by analyzed and the properties of the observed photospheric features can be studied. Thorough analysis and interpretation of the reduced GCT data will be presented in forthcoming sections in Chapter 3.

Here, we demonstrate the quality of the seeing conditions during the observations and the quality of the recorded and reduced data by presenting 2D intensity maps in continuum and line core of Stokes $I$ and magnetograms from the wing of Stokes $V$ profiles (Fig. 2.6). Continuum was selected between the telluric line $\mathrm{O}_{2} 6302.0 \AA$ and $\mathrm{Fe}$ I $6302.5 \AA$. The line core map is in the center of the Fe I $6302.5 \AA$ line. A distribution of the magnetic field was obtained in first approximation from the polarimetric data. For each spatial position the corresponding Stokes $V$ profile was integrated over a spectral window of $30 \mathrm{~m} \AA$ width which was set at $-75 \mathrm{~m} \AA$ from the center of the 6302.5 line.

Intensity maps were constructed from a sequence of 1D spectrograms, which were exposed while the image in the field of view of the telescope was scanned across the spectrograph slit. Since the spatial sampling is different in the direction parallel and perpendicular to the slit, images were resampled to the common (smallest) value of $0.19^{\prime \prime}$. Finally, residual artifacts were filtered out and maps were smoothed with a $3 \times 3$ pixels boxcar.

Images in the upper row of Fig. 2.6 are constructed from the spectrograms observed on 08.06.01 with a slit width 0.75 , exposure time $0.2 \mathrm{~s}$, number of steps of the scanner 60 , and FOV $27^{\prime \prime} \times 40^{\prime \prime}$.

Images in the lower row: spectra observed on 09.08.01 with the slit width $1^{\prime \prime}$, exposure time $0.4 \mathrm{~s}$, number of steps of the scanner 40 , and FOV $27^{\prime \prime} \times 27^{\prime \prime}$. 

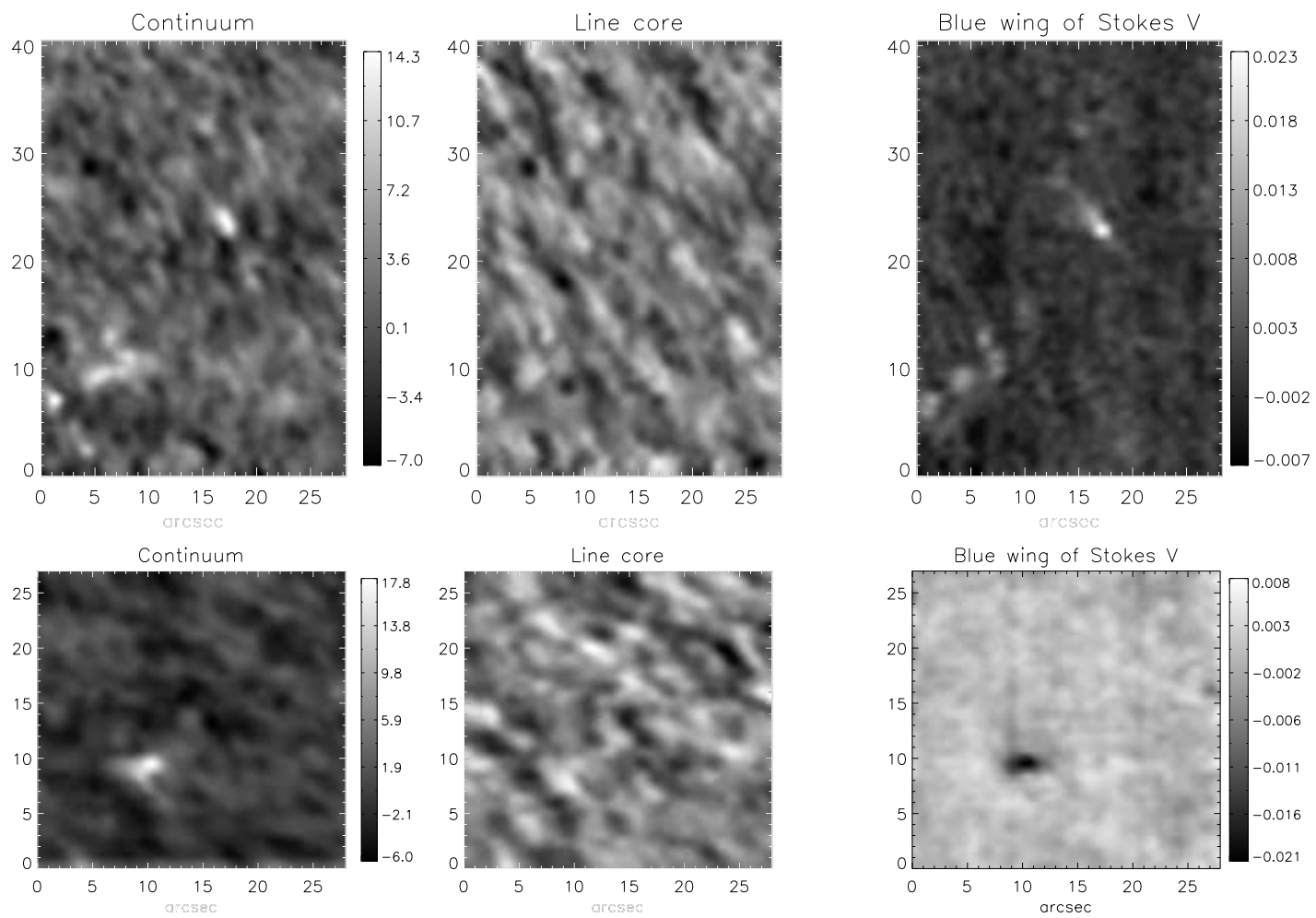

Figure 2.6: Examples of intensity maps constructed from reduced 1D GCT spectrograms, which contain polar faculae. Continuum images are in $(I-<I>) /<I>$ units. Maps in the line core and in the wing of Stokes $V$ are in the $\lambda \mathrm{Fe}$ I $6302.5 \AA$ spectral line. Stokes $V$ maps are in $V / I_{Q S, C}$ units. For more details see the text. 


\subsection{Two-dimensional spectra observed with Vacuum Tower Telescope}

The spectropolarimetric observations with the GCT were complemented by high resolution spectropolarimetric and white-light speckle observations with the VTT. They were obtained with the two-dimensional spectrometer based on wavelength scanning FabryPerot interferometers operated by the Göttingen solar physics group.

The observational data used in this work were taken on 15 June, 2001, and on 28 and 29 April, 2002. Again, as for the observations with the GCT high latitude areas containing PFs were recorded.

Spectrograms were taken with the VTT in the same spectral ranges as with the GCT. The magnetic sensitive iron lines Fe I 6302.5 $\AA$ ( $g=2.5)$ and Fe I $6301.5 \AA(g=1.67)$ were observed. Additionally, spectropolarimetric data in the line of ionized iron Fe II $6149.3 \AA$ $(\mathrm{g}=1.33)$ were obtained. This line is much weaker than the above lines of neutral iron. But the following peculiarities make this line interesting for magnetic field studies:

- it forms in lower layers of the photosphere in comparison with the neutral iron lines;

- it has a different temperature dependence than the other two lines above;

- due to the peculiar configuration of its Zeeman pattern the line does not produce linear polarization, the $Q$ and $U$ parameters of the Stokes vector are equal to 0 .

The specifications of the observations with the VTT are:

- 2D “Göttingen” FPI spectrometer,

- spectral resolution $\lambda / \Delta \lambda \sim 140000$,

- CCD camera La Vision with size of $384 \times 286$ pixel,

- sampling in spatial direction $\Delta x=0$ ". 105 (corresponding to the pixel distance of the CCD detector),

- Stokes $V$ polarimeter: $\frac{1}{2}(I+V)$ and $\frac{1}{2}(I-V)$ are recorded,

- observed data: 3D data cube $I(x, y, \lambda)$ with size $\sim\left(15^{\prime \prime}, 25^{\prime \prime}, 0.5 \AA\right)$,

\subsubsection{Fabry-Perot interferometer}

The optical setup of the "Göttingen" FPI spectrometer is schematically presented in Fig. 2.7. It consists basically of two channels, broadband (BB) and narrowband (NB). The beam splitter BS splits the light into the two channels in the following proportions: $5 \%$ for BB and $95 \%$ to NB. Optionally, a third G-band channel can be used but it will need a CCD camera sensitive to blue light. In the BB channel the central wavelength and the width of spectral range is set by an interference filter IF1 with typical FWHM of 50 $100 \AA$ A. The NB channel of the setup is, in its essential parts, equipped with a Stokes $V$ polarimeter VP and the spectrometer which includes two Fabry-Perot etalons and an interference filter IF2. More details about the principles of the functioning of the FPI spectrometer can be found in Bendlin et al. (1992), Volkmer (1995), Bendlin \& Volkmer (1995), and Koschinsky (2001). 


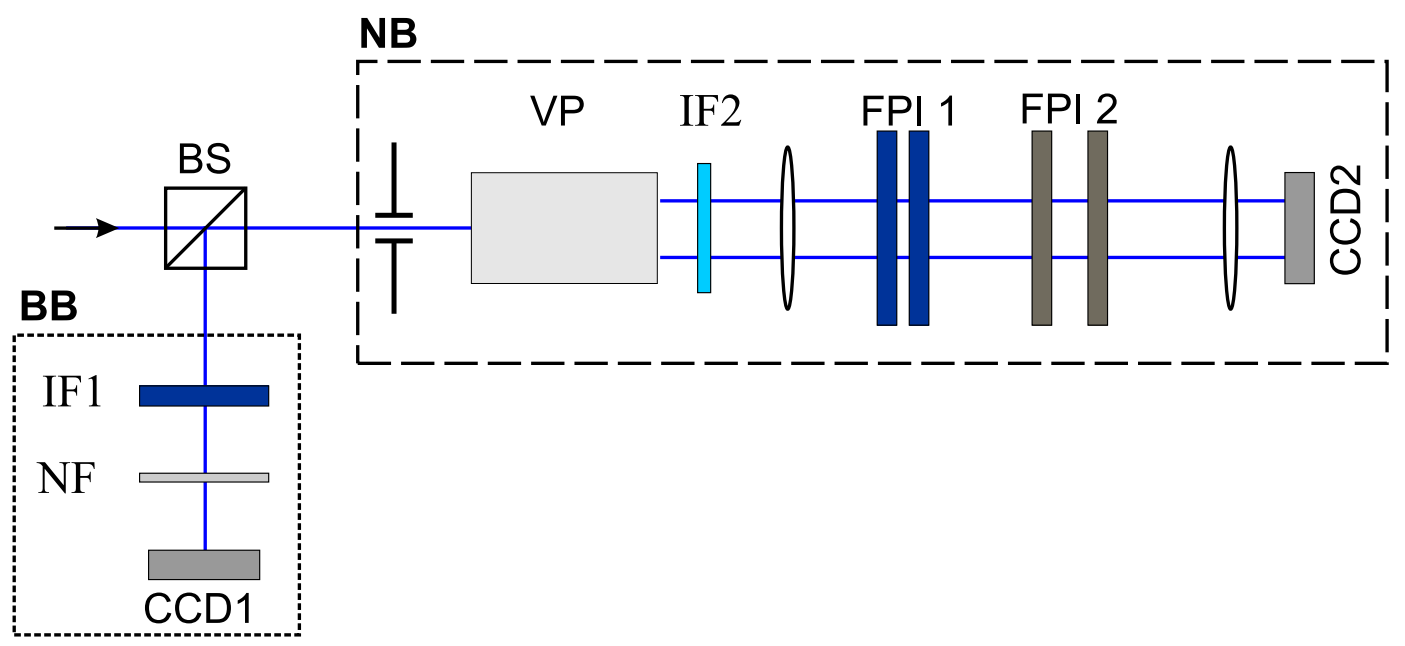

Figure 2.7: Optical setup of the FPI spectrometer, schematically

Here we only mention that the "Göttingen" FPI is mounted in the parallel (collimated) beam. Another possible mounting, the telecentric mounting is realized in TESOS (Kentischer et al. 1998). The discussion about both mountings, their advantages and disadvantages was presented by Kneer and Hirzberger (2001). Mounting in the collimated beam is free of the orange peel pattern, typical for telecentric mounting and caused by flatness imperfections of the etalons, while mounting the FPIs in the parallel beam changes the wavelength of maximum transmission across the field of view, producing the so called blueshift

$$
\Delta \lambda \approx-\lambda_{0} \frac{\theta^{2}}{2}
$$

where $\theta$ is the angle of incidence of light, which is different for different positions in the field of view and $\lambda_{0}$ is the wavelength of the maximum of transmission at normal incidence. The change of the position of the maximum of transmission is equivalent to the shift of the line profile. More details about the origin of the blueshift and methods of its correction can be found in the theses of Koschinsky (2001) and Janssen (2003).

What is important for the data reduction and interpretation is the knowledge about the transmission function of the spectrometer. The sought function is given by multiplication of three functions: transmission of the interference filter (FWHM $=8 \AA$ ), of Fabry-Perot etalon $1(\mathrm{FWHM}=0.53 \AA)$ and of Fabry-Perot etalon $2(\mathrm{FWHM}=0.044 \AA$ ). The interference filter serves for the selection of the desired order of the broadband FPI, which in turn selects the order of the narrowband FPI (Fig. 2.8 upper frame). The FWHM of the resulting transmission of the spectrometer will be equal to the FWHM of the narrow one.

Since, mathematically, the actions of three filters are multiplicative it does not matter in which order they are mounted on the optical bench. The whole transmission of the spectrometer acts on the observed spectral range as a convolution kernel. 

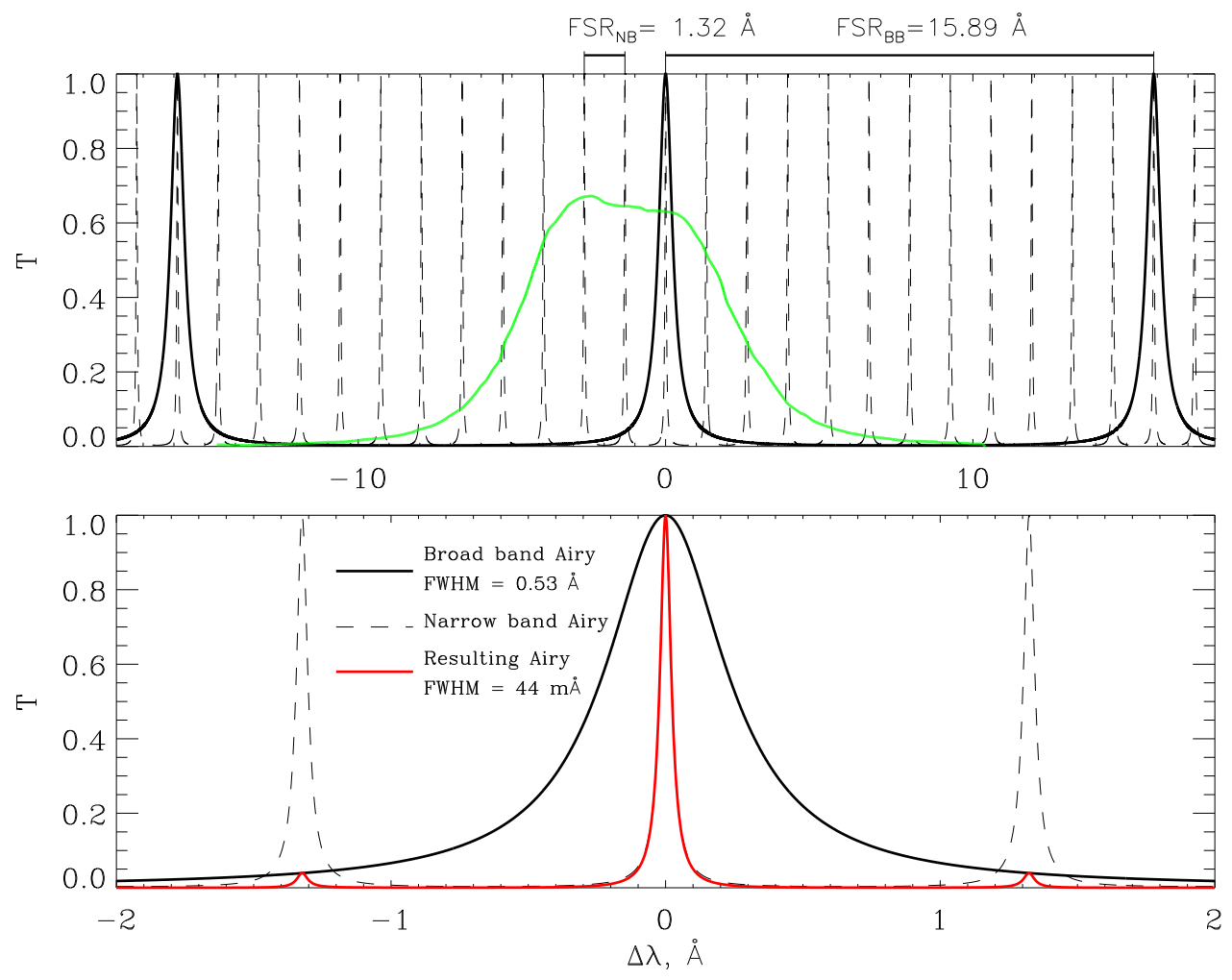

Figure 2.8: Transmission of the spectrometer. Upper panel: transmission of the used interference filter (green curve), Airy function of the broadband FPI (black solid line), Airy function of the narrowband FPI (dashed line). Lower panel: resulting transmission curve of the spectrometer with secondary maxima. FSR and FWHM denote the Free Spectral Range and the Full Width at Half Maximum, respectively.

\subsubsection{Structure of observations}

In this section we discuss peculiarities of observations with the 2D spectrometer and describe a typical observational procedure.

The observed data are organized in scans. Each single scan consists of two image sequences: one recorded in the BB channel and the other one in the NB channel. The images in both channels are exposed simultaneously. The broadband channel is used for speckle interferometry, and the narrowband channel for 2D spectrometry. Speckle interferometry sets the requirements on the duration of the scan and the exposure time of the single image: a) the exposure time must be short enough to consider the seeing conditions as being frozen; b) a duration of the burst must be shorter than the characteristic time of changes in the photosphere, so that all images in one burst are taken under different seeing condition but of the unchanged object. The last requirement is important also for the NB data. A spectral line is scanned in time, so the observed structures should not evolve between the first and the last exposure in the NB channel. In our case the exposure time was $20-30 \mathrm{~ms}$ and one scan lasted typically $50 \mathrm{~s}$.

The maximum number of images in one burst is limited to 150 by the currently used setup. The use of faster computers and CCD cameras and software without memory limitation will allow to record more images with shorter exposure and in shorter time and in such a way to improve the quality of data reduction. 
Table 2.2: Description of the obtained observational sets.

\begin{tabular}{cccccccc}
\hline Date & $\begin{array}{c}\text { Observed } \\
\text { Line }\end{array}$ & $\begin{array}{c}\text { Exposure } \\
\mathrm{ms}\end{array}$ & $\begin{array}{c}\Delta \lambda \\
\mathrm{m \AA}\end{array}$ & $\begin{array}{c}\text { FWHM } \\
\mathrm{m \AA}\end{array}$ & $\begin{array}{c}\text { Im./pos. } \\
N_{s p}\end{array}$ & $\begin{array}{c}\text { Spectral range } \\
\mathrm{m} \AA\end{array}$ \\
\hline 15.06 .2001 & 6149.3 & 30 & 16.5 & 46.7 & 5 & 30 & 480 \\
28.04 .2002 & 6302.5 & 20 & 25 & 44 & 7 & 21 & 500 \\
29.04 .2002 & $\begin{array}{l}6301.5 \\
6302.5\end{array}$ & 30 & 31.5 & 44 & 5 & 28 & $\begin{array}{c}410+ \\
410\end{array}$
\end{tabular}

In the BB channel all images are exposed in the same spectral range defined by the interference filter IF1, and are used for further speckle reconstruction. The output from the reduction of the BB images will be one single reconstructed image. In narrowband all exposed images are distributed between several spectral positions. The scanning along wavelength is carried out by the FPI spectrometer. To reduce the noise and to accumulate the signal several images per spectral position are taken (Im./pos. in column 6 of Tab. 2.2). For polarimetric observations the typical number of images per position is $5-10$. This implies that the maximum possible number of observed spectral positions $\left(N_{s p}\right)$ will be 150 / [Im./pos.]: 30 and 15, respectively. The output from the reduction of the NB images will be $N_{s p}$ two-dimensional narrowband filtergams corresponding to the wavelength positions along the scanned line.

The width of the scanned spectral range is defined by $N_{s p}$ and by the step of the scanning interferometer or, in other words, by the spectral sampling of the spectrometer $\Delta \lambda$. All mentioned parameters of scanning (Im./pos., $N_{s p}$ and $\Delta \lambda$ ) are set manually by the observer. Their choice depends on the goal of the observations.

High SNR can be achieved by increasing the number of Im./pos. at the expense of the width of spectral range. In turn the scanned spectral range is set by a proper selection of $\Delta \lambda$. The reasonable choice of $\Delta \lambda$ (to avoid too much oversampling and undersampling) is a value close to the half of FWHM of the spectrometer. Because of the limitation to 150 images in one scan the maximum spectral range which can be scanned without undersampling is about $0.5-0.6 \AA$, which typically corresponds to one spectral line.

The data used in this work were obtained on three different days and with different sets of parameters of the spectrometer (Tab. 2.2). On 29.04.2002 two spectral lines were recorded in one scan. It was achieved by some undersampling and subsequent scanning of each line and a jump of the position of maximum transmission between the lines.

\subsubsection{Spectral quality of 2D spectrograms}

The estimation of the spectral quality of the data is very important for further interpretation of the observations. We have proceeded in the same way as described in Sect. 2.1.1. Frames with flat fields observed in the narrowband near the center of the solar disk were corrected for dark offset, averaged across the field of view and compared with the reference line profiles extracted from the high spectral resolution Fourier Transform 

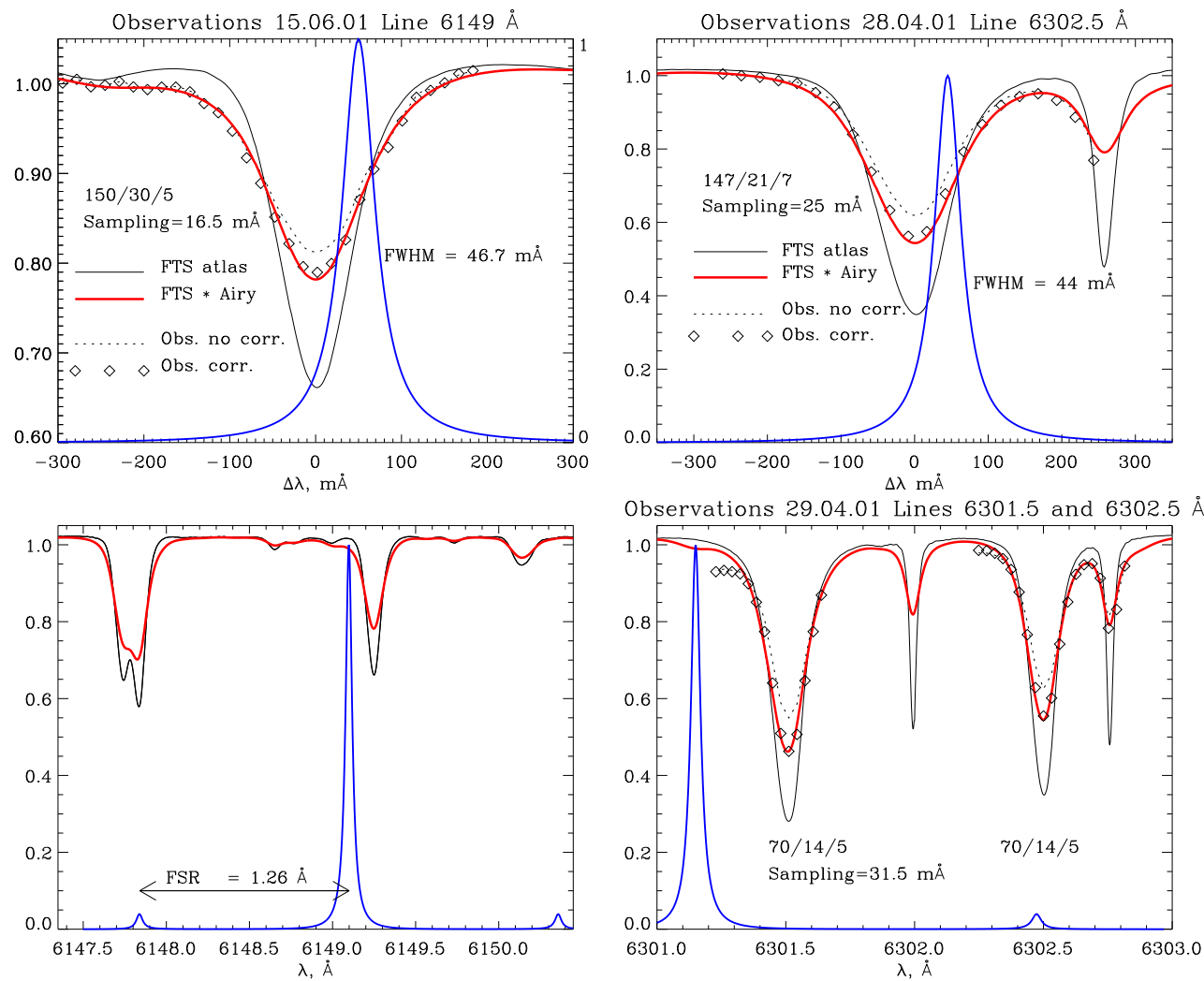

Figure 2.9: For each of the three days of observations the recorded line profiles (dotted lines) were corrected for IR and stray light contamination (open squares). They are compared with the reference line profiles taken from the FTS atlas (solid black line) convolved with the Airy function (blue line) of corresponding FWHM. The result of the convolution is coded with red color and is in good agreement with the observed profiles. The lower left panel corresponds to the observation taken on 15.06.2001 and demonstrates the effect of secondary maxima of the transmission. The small gap in the blue wing of the Fe II $6149 \AA$ line is due to the contribution of two neighboring spectral lines at the distance of one FSR.

Spectrometer (FTS) atlas by Brault and Neckel (quoted by Neckel 1999). All spectral lines observed with the $2 \mathrm{D}$ spectrometer appear to be much weaker than the reference lines (Fig. 2.9 dotted and solid lines). The measured line depressions are almost two times smaller than the depressions of the corresponding unbroadened lines (Tab. 2.3). This is mainly due to the broadness of the transmission of the used spectrometer.

\begin{tabular}{ccc}
\hline Line & Measured & FTS \\
\hline 6149.3 & 0.19 & 0.32 \\
6301.5 & 0.45 & 0.72 \\
6302.5 & 0.35 & 0.65
\end{tabular}

Table 2.3: Spectral line depressions measured in averaged flat field profiles in comparisson with the depressions of the reference lines extracted from the FTS atlas. 
A realistic transmission function (Fig. 2.9 blue line) was constructed by multiplication of the Airy functions of the broadband FPI with that of the narrowband FPI. The FTS lines were convolved with this kernel to simulate the influence of the spectrometer on the observed profiles. The result of convolution (Fig. 2.9 red line) is much closer to the recorded lines but there still remains some deficit of measured line depressions. Likewise, as in the case of the 1D spectrograms observed with the grating spectrograph (Sect. 2.1.1) this discrepancy can be explained by contribution of the IR background and scattered light in the spectrograph, which gives a constant offset of counts of the detector. It can be estimated and subtracted from the observed line profiles. In this way the corrected profiles (Fig. 2.9 open squares) are in good agreement with the reference. The found offsets are then applied also to the data.

An important peculiarity of the transmission function of the spectrometer which should be taken into account is the presence of secondary maxima. Secondary maxima appear due to the fact that the extension of the wings of the Airy function of the broadband FPI exceeds the FSR of the narrowband etalon (Fig. 2.8 lower panel). If the observed spectral line has no other lines near it at the distance $\Delta \lambda<1.3 \AA$ then the convolution of the secondary maxima with the flat continuum will give a constant, wavelength independent contribution to the observed line profile and the effect will be similar to the contribution from the scattered light, which can be easily estimated and corrected. But if at the distance of the FSR there is another strong line it will change the shape of the observed line profile and create gaps. An example of such a gap can be seen in the blue wing of the $\lambda 6149.3 \AA$ line (Fig. 2.9). Moreover, if the neighboring spectral line is magnetically sensitive then the secondary maxima will cause a contamination of the observed Stokes $V$ profiles by the polarimetric information of the neighboring spectral line.

\subsubsection{Broadband speckle reconstruction}

The uniqueness of solar observations is that the Sun is simultaneously the object of the study and the disturber of the observations. The Sun heats the air, causing turbulent convection, which leads to various distortions, called seeing. For the correction of the observations methods of speckle-interferometry were used. The idea of the method is to take a sequence of so called speckle images with the exposure time short enough to consider the seeing conditions as being frozen. The set of images of the same object but obtained under different atmospheric conditions allows restoration by means of speckle reconstruction - a statistical approach intended to remove the degrading influence of the Earth's atmosphere.

A sequence of broadband (FWHM = 50 $\mathrm{A}$ ) images was used for speckle reconstruction. All images are divided into subimages of the size of the isoplantic patch, areas within which identical seeing conditions prevail. To each isoplanatic patch corresponds its own optical transfer function (OTF). The restoration is applied to each single patch separately. The image reconstruction takes place in Fourier space, where the images are separated into amplitudes and phases, and each of them is restored separately. The amplitudes are corrected using the spectral ratio method (von der Lühe 1984). The corrected phases are obtained from the speckle masking method (Weigelt 1977). A detailed description of the method can be found in the thesis of de Boer (1993). 

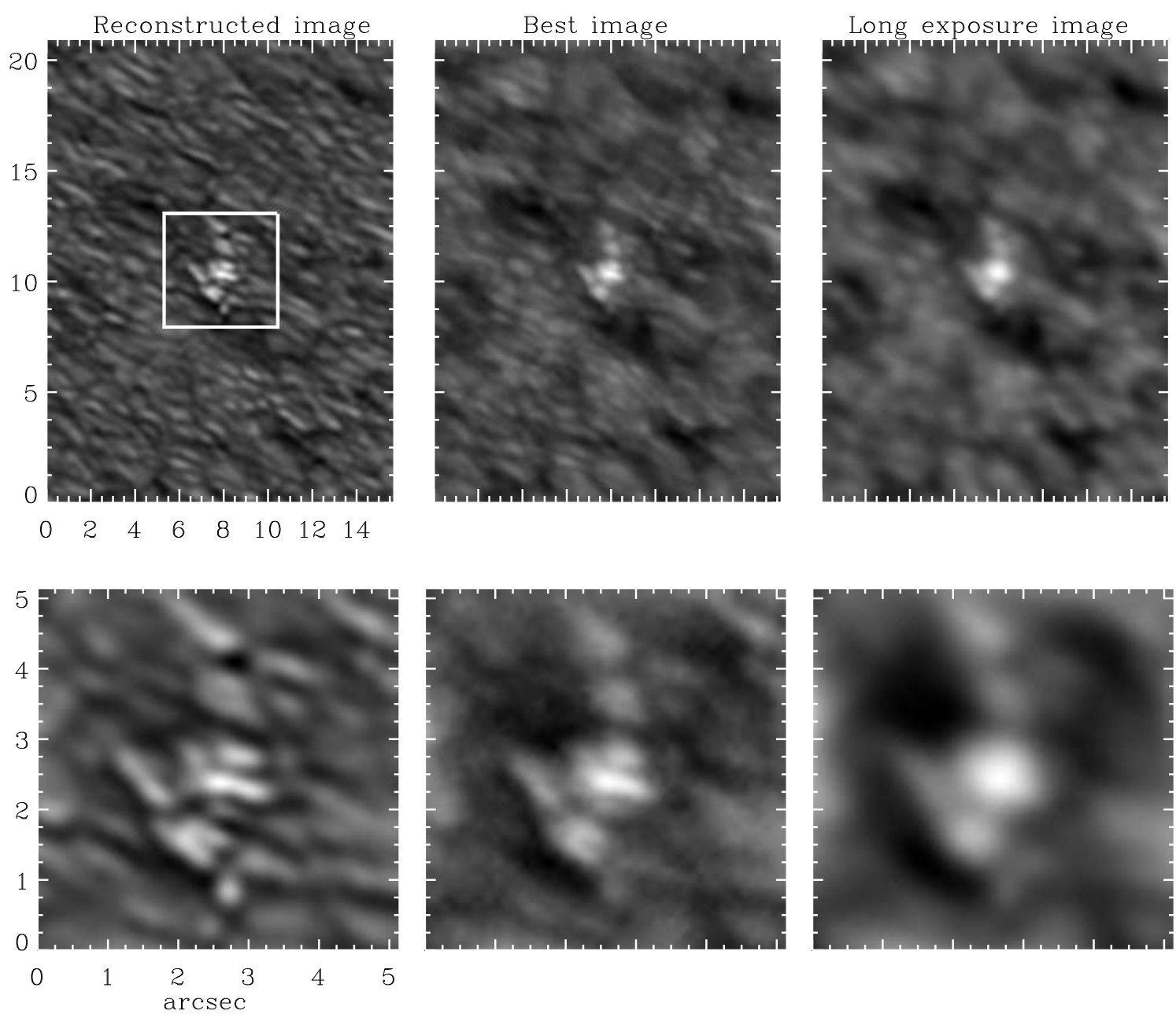

Figure 2.10: Example of a reconstructed broadband image of a polar facula in comparison with the best quality image in the speckle sequence and the long exposure image. The upper row shows the whole field of view, the white square outlines the magnified part of the image presented in the lower row.

The computer code for speckle reconstruction is written in Interactive Data Language (IDL). It was developed in the Universitäts-Sternwarte Göttingen and is used for this work without any changes. The code calculates the normalized Fried parameter, or seeing parameter $\alpha=r_{0} / D$ - a quantitative characteristic of the seeing and of the quality of the image, where $r_{0}$ is the Fried parameter and $D$ is the aperture of the telescope $(70 \mathrm{~cm}$ for the VTT). Observations characterized by $\alpha<0.12$ are considered not worth reconstruction since seeing was bad. Values of $0.14<\alpha<0.18$ are typical for Izaña and correspond to moderate/good seeing condition, data with $\alpha>0.20\left(r_{0}>14 \mathrm{~cm}\right)$ were taken under excellent seeing conditions, and already raw images without restoration exhibit high contrast and demonstrate very high quality for ground based observations. Application of the speckle reconstruction method to such images will provide diffraction limited images free of atmospheric distortions (Fig. 2.10). 
Two tricks to improve the quality of the reconstructed images observed near the limb were applied:

- removing the bad speckle images: sorting all speckle images in a burst in order of descending quality, and taking only the first best 100 or 120 images instead of all 150;

- use of many flat fields: flat field frames are used in the speckle reconstruction code for two purposes, for flat fielding of the data and for estimation of the power spectrum of the noise for further construction of the noise filter. Ideal flat fields should contain no intensity variation from the solar structures, it can be achieved by sufficient averaging of many flat field exposures. By default the code uses for averaging only one file containing typically 150 flat field frames. Averaging of more files, say 10, improves the quality of reconstruction.

\subsubsection{Reconstruction of narrowband images}

The reconstruction of the images from the narrowband channel of the Fabry Perot spectrometer was made following Keller and von der Lühe (1984). In this section we give a brief description of the method. More details can be found in Krieg (1999), Koschinsky (2001), and Janssen (2003).

Each single filtergram can be reconstructed knowing the corresponding optical transfer function (OTF). An estimate of the instantaneous OTF for each exposed narrowband image can be found using the simultaneously exposed images from the broadband channel and the speckle reconstructed image. The wavelength dependence of the OTF is neglected. Hence, the problem of reconstruction of the narrowband filtergrams is reduced to the deconvolution with a known kernel. Deconvolution is equivalent to division in Fourier domain. The problem of deconvolution is, as all inverse problems, very sensitive to noise. Therefore efficient filtering should be applied, the optimum filter described by Brault and White (1971) is used. Finally, the expression for the restoration and filtering of the narrowband images is

$$
O_{n}(\boldsymbol{q})=H \cdot \frac{\sum I_{n}(\boldsymbol{q}, t) I_{b}^{*}(\boldsymbol{q}, t)}{\sum\left|I_{b}(\boldsymbol{q}, t)\right|^{2}} \cdot O_{b}(\boldsymbol{q}) .
$$

Here, $O_{n}$ is the reconstructed narrowband image in Fourier space, $H$ is the optimum filter, $I_{b}$ and $I_{n}$ are the broad- and narrowband observed images, respectively, and $O_{b}$ is the reconstructed broadband image. The asterisk ${ }^{(*)}$ denotes complex conjugation. The summation runs over the images taken at identical wavelength settings of the spectrometer. The optimum filter $H$ removes the noise at the expense of cutting high spatial frequencies in Fourier space and, thus, limits the spatial resolution of spectropolarimetric data typically to $0.5-0.7$. The achieved spatial resolution is lower than the resolution of the reconstructed broadband images but is very good for ground based polarimetry in the narrowband channel (Fig. 2.11). The computer code was developed in the Universitäts-Sterwarte. It was recently revised, in many parts rewritten and adapted to the reconstruction of polarimetric observations by Janssen (2003).

As it was mentioned several times, limb observations, due to the low count level and the higher sensitivity to noise, usually require very accurate treatment. Here, an improvement of the data reduction was again achieved by an additional correction of the flat field frames. Images with flat fields exhibit exactly the same instrumental effects as the data 


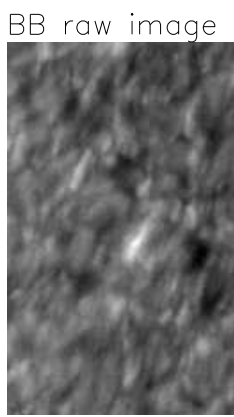

Blue wing

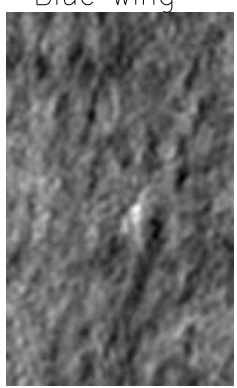

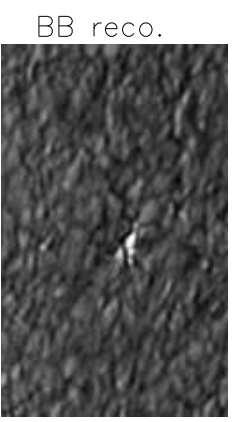

Line core

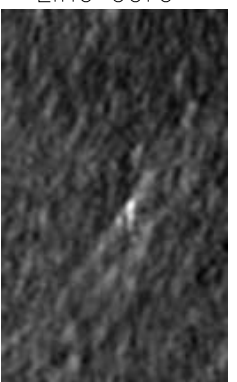

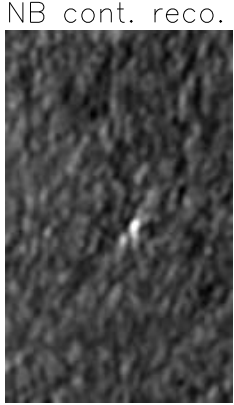

Red wing

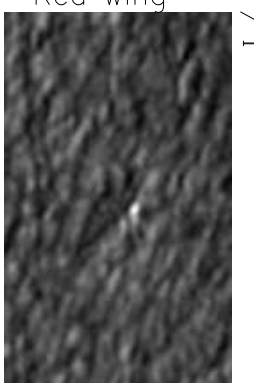

Fe I $6302 \AA$

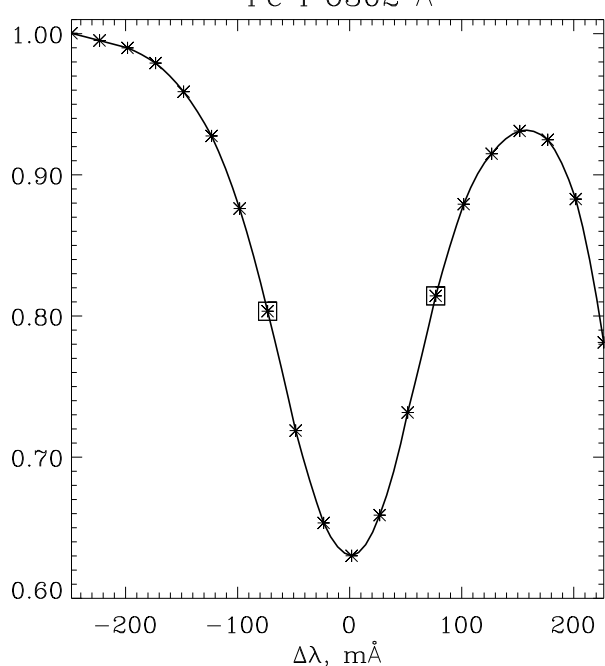

Figure 2.11: Example of the reconstructed 2D spectrograms of a polar facula observed in Fe I $6302.5 \AA$ line. Narrowband filtergrams taken at different spectral position are presented in the lower row, the image exposed in the continuum of spectral line is compared with the broadband reconstructed frame and corresponding raw image in the upper row. Squares on the average line profile (right panel) indictae the spectral positions of the presented filtergrams in the red and the blue wing.

images and should be corrected. Observations obtained with a FPI mounted in the collimated beam contain a shift of the maximum FPI transmission across the field of view. It has two consequences for the observed spectrorams: relative shifts of spectral lines themselves and variation of the intensity across the field of view caused by such shifts. For the 2D filtergrams obtained in the continuum near a spectral line the intensity does not change with $\lambda$ and the shift of the maximum transmission will not lead to any intensity variations across the field of view. The same holds for the core of the line where the intensity gradient is close to zero. At such wavelengths the effect is negligible.

The intensity gradient in a line profile reaches its maximum in the wings, where the second derivative vanishes. Therefore images taken at such wavelengths will exhibit the most intensity variation across the field of view (Fig. 2.12). The data images are correted for this effect, but the important point here is that the frames containing the flat fields must also be corrected for the wavelength variation across the field of view before the procedure of flat fielding of the data. It improves the quality of the reconstruction. This correction is especially important for strong lines where the intensity gradient in the wings is very strong. Moreover, wavelengths where $\left|\frac{d I}{d \lambda}\right|=\max$ correspond approximately to the positions of the lobes of Stokes $V$, therefore these artificial intensity fluctuations will affect most of all polarimetric signals, the most interesting one in the context of studying small-scale magnetic fields. 


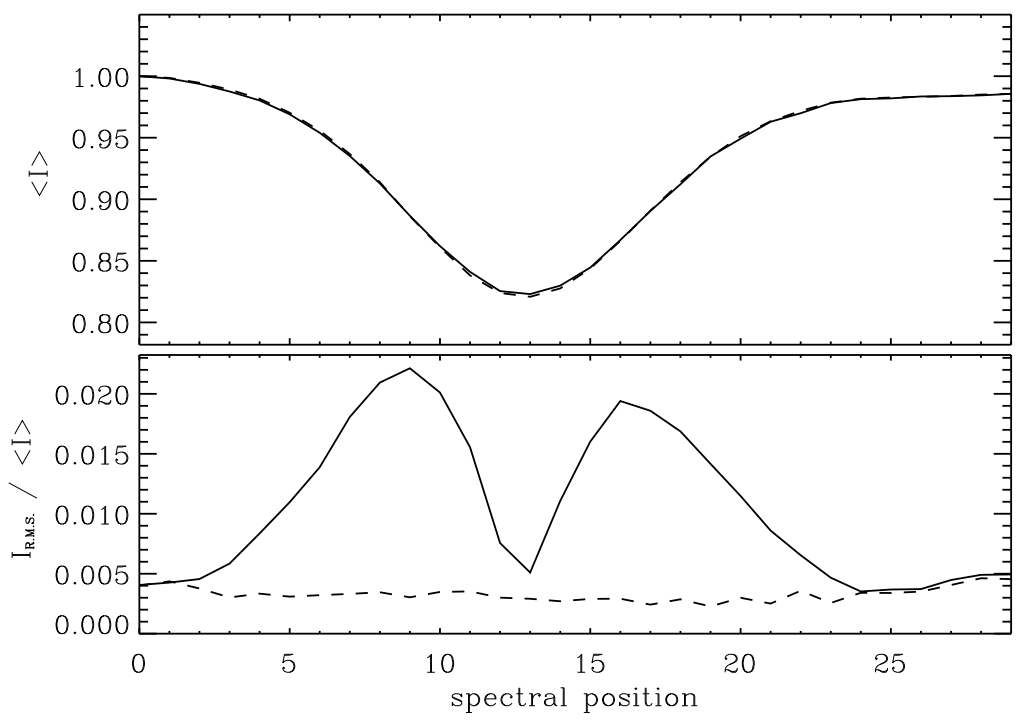

Figure 2.12: Correction of the flat fields for blueshift across field of view. Upper panel: averaged intensity for different spectral positions before correction (solid line) and after (dashed line). Lower panel: the same but for r.m.s variations of the intensity across field of view. The high contrast of the uncorrected flat fields (solid line) is caused by the variation of the intensity across the field of view produced by non-constant wavelength position of the maximum transmission. The effect is strongest in the line wings, where the intensity gradient is largest.

\subsubsection{Stokes $V$ corrections}

The reconstruction code together with various corrections are applied to the spectrograms containing left and right circularly polarized light $\frac{1}{2}(I+V)$ and $\frac{1}{2}(I-V)$ separately and independently from each other. The further summation and subtraction of the images from the two channels will result in $I$ and $V$ spectrograms, respectively. Since the images corresponding to different spectral position were exposed at different moments of time under changing seeing conditions, and were reconstructed separately, the obtained spectrograms are very noisy in the wavelength direction. Especially the $V$ spectrograms, as differences of low count images, suffer from noise. The amplitude of the noise (for the obtained data typically $0.8-1 \%$ of the continuum intensity) essentially limits the polarimetric sensitivity of the data. But apart of this unavoidable random noise the spectrograms contain systematic trends along $\lambda$, which also must be corrected.

One of the calibration applied to the spectrograms is the correction, already mentioned above, for wavelength changes of the maximum transmission of the spectrometer. In the used setup the polarimeter was mounted before the FPI. So, the light was first split into two beams, which afterwards entered the FPI under different incidence angles, and while passing through the spectrometer exhibited wavelength changes. As a result line profiles in each beam are shifted differently. Therefore, the correction of wavelength shifts in both channels must be done very carefully because even small wavelength displacements between left and right channels will result in a systematic artificial Stokes $V$ signal. 
To check the correction, we have compared intensity profiles averaged across the field of view in the left and right beams. The data under consideration were taken at high latitudes far from active regions. Although the spectrograms contain PFs, structures with presumably strong magnetic fields, they should exhibit vanishing average magnetic flux density from the whole field of view with size of $\simeq 15^{\prime \prime} \times 20^{\prime \prime}$. PFs occupy a very small fraction of the averaged area and their contribution to the flux is negligible. Network and intranetwork fields due to the mixed polarities and small filling factors will neither contribute to the magnetic flux. Therefore averaging across the field of view should produce practically identical and unshifted intensity profiles in the left and the right channel. The relative shift was estimated and all line profiles in one of the channels were shifted.

But even after the correction for shifts there still remains a systematic part, which can be clearly seen in the averaged $V$ profile (Fig. 2.13 left panel). The correction would be in the subtraction of the found residual systematic trend from the Stokes $V$ profiles in each pixel. This correction is similar to the second order flat fielding described by Sánchez Almeida \& Martínez Pilet (1994) and which was already used for the correction of this kind of data by Janssen (2003) in the way that an unwanted trend is subtracted from Stokes $V$. The difference here is that for the estimation of systematic trends we use instead of flat fields the data themselves, supposing that the averaging across the field of view removes any magnetic signal.
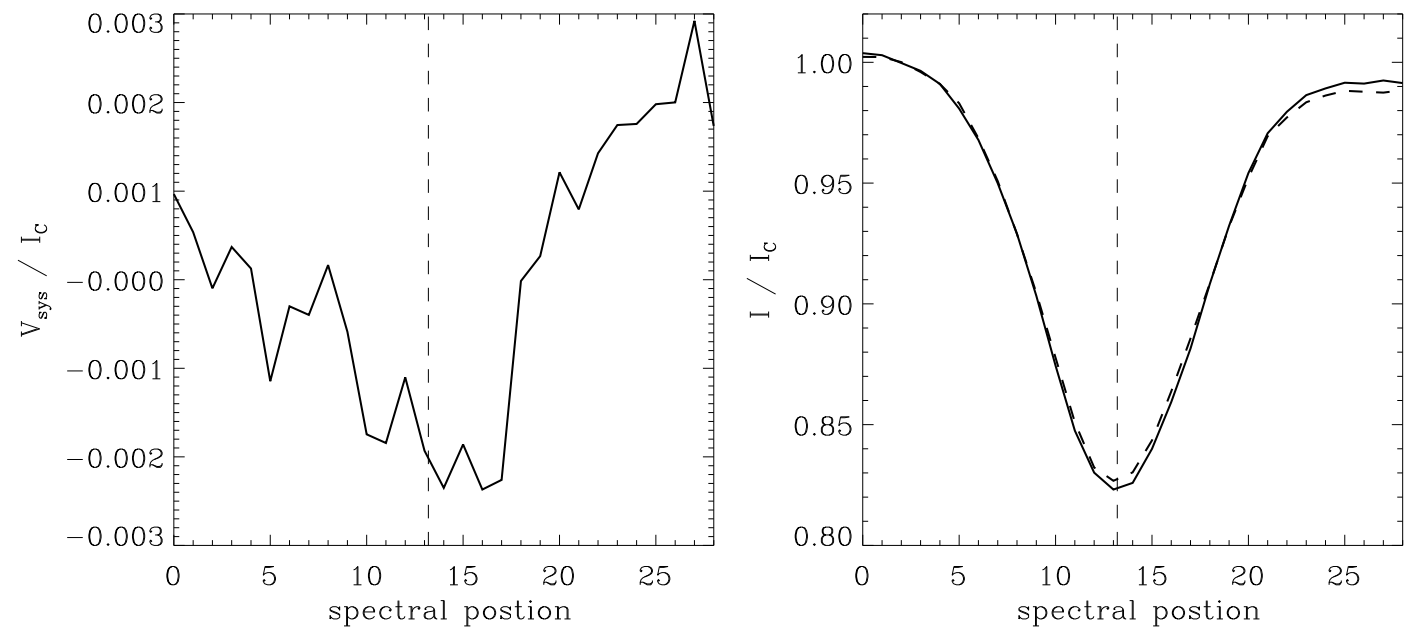

Figure 2.13: Left panel: systematic error in Stokes $V$ signal, obtained by averaging across the field of view of the spectrogram. Right panel: averaged intensity profiles in the left and right beams are corrected for the relative shift but still show differences at certain wavelengths. Vertical dashed line denotes position of the line center.

This correction can also be done in another way. Intensity profiles averaged in both channels still remain slightly different even after correction for their relative shift (Fig. 2.13 right panel). The discrepancy again is a result of different paths of the left and right beams through the spectrograph and of the independent treatment of filtergams in the left and right channels. Now, the calibration would be in the multiplication of profiles in each channel with the correction curve found from the averaged profiles. 


$$
\begin{aligned}
& I_{l}^{1}(x, y)=I_{l}^{0}(x, y) \frac{<I_{l}^{0}>+<I_{r}^{0}>}{2<I_{l}^{0}>} \\
& I_{r}^{1}(x, y)=I_{r}^{0}(x, y) \frac{<I_{l}^{0}>+<I_{r}^{0}>}{2<I_{r}^{0}>}
\end{aligned}
$$

Here, superscipts ${ }^{(0)}$ and ${ }^{(1)}$ denote spectrograms before and after correction, respectively, and the averaging was performed over the field of view.

After the described calibrations the averaging in the left and right channels will result in completely identical intensity profiles and Stokes $V$ will be free of systematic errors. The only unwanted part which remains in the reduced data is random noise in wavelength direction, which requires additional treatments. 


\subsection{Signal recognition in polarimetric data}

In the previous sections we have described the reduction of the polarimetric data: 1D spectrograms, observed with the slit spectrograph at the GCT and 2D spectrograms obtained at the VTT with the FPI spectrometer. The reduction and characteristics of the data in both sets are very different but in both cases we stopped the reduction at the point when spectrograms contain only useful signal and residual noise after the filtering of the spectrograms in the left and right beams. For example, the resulting reduced 1D Stokes $V$ spectrogram (Fig. 2.5 right frame) is free of all kind of distortions and contains only the signal and partly reduced noise. The same holds for the 2D spectrograms observed with the VTT. The narrowband filtergrams were corrected for atmospheric influence and filtered in spatial direction, additional calibration removes systematic trends in wavelength direction, but there still remains noise, setting the threshold on the lowest detectable amplitude of the Stokes $V$ signal. Allthough the nature of noise is different in the two kinds of data under consideration, the treatment of noise described in this section will be applied in the same way to both kinds of spectrograms.

Additional noise filtering in wavelength direction is possible in principle but will lead to degradation of the resolution. An alternative approach is the application of so called signal recognition algorithms. The idea is to construct an analytical function $Y(\lambda, \mathbf{a})$ controlled by a vector of free parameters a which is able to fit the shape of Stokes $V$ profiles, and to find by a non-linear least squares method the parameters which give the best fit $\left(\chi^{2}(\mathbf{a}) \rightarrow \min \right)$ of the analytical function to the observed noisy $V$ signal:

$$
\chi^{2}(\mathbf{a})=\sum_{\lambda_{i}}\left(V_{\mathrm{obs}}\left(\lambda_{i}\right)-Y\left(\lambda_{i}, \mathbf{a}\right)\right)^{2} .
$$

The free parameters derived in such a way will unambiguously define the function $Y(\lambda)$ which will be considered as a sought noise-free Stokes $V$ signal and will be used for further analyses of the polarimetric data. As a function able to reproduce typical shapes of $V$ profiles without magneto-optical effects we have chosen the linear combination of two Gaussian functions and a parabola, a function of eight free paramteres: $\mathbf{a}=\left[a_{0}, a_{1}, a_{2}, a_{3}, a_{4}, a_{5}, a_{6}, a_{7}, a_{8}\right]$.

$$
Y(\lambda, \mathbf{a})=a_{0} e^{-\left(\lambda-a_{1}\right)^{2} /\left(2 a_{2}^{2}\right)}+a_{3} e^{-\left(\lambda-a_{4}\right)^{2} /\left(2 a_{5}^{2}\right)}+a_{6}+a_{7} \lambda+a_{8} \lambda^{2}
$$

The second order terms are used to achieve a better fit. Real polarimetric observations usually contain various impurities, such as crosstalk from $I$ to $V$, imperfections of flat fielding, systematic errors. The sources of it and the ways to correct were discussed in previous sections. For good quality or properly corrected observations only the linear term or even only the constant offset can be left for a faster convergence of the fitting algorithm.

For minimization of the $\chi^{2}(\mathbf{a})$ function a subroutine CURVEFIT from the standard IDL software distribution was used. It uses a gradient-expansion algorithm to compute a non-linear least squares fit to a user-supplied function with an arbitrary number of parameters. The user-supplied function may be any non-linear function where the partial derivatives are known or can be approximated. Iterations are performed until the changes of $\chi^{2}$ are smaller than a specified amount or until a maximum number of iterations have been performed. 

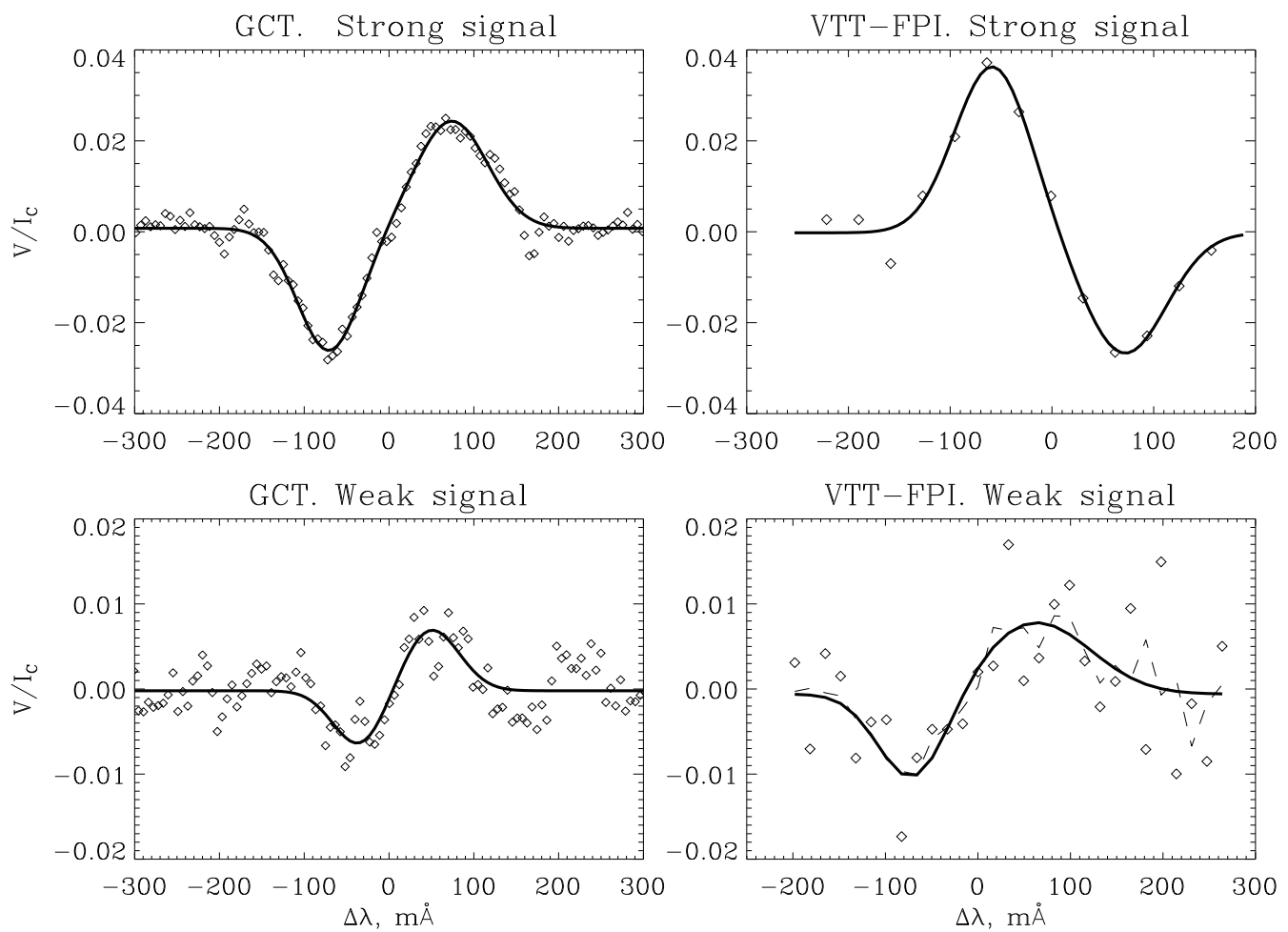

Figure 2.14: Examples of non-linear least squares fits of a linear combination of two gaussian functions (solid lines) to typical strong and weak Stokes $V$ profiles (open squares), observed with different spectral resolution, sampling and SNR. On the lower right panel the dashed line results from smoothing of the observed noisy profile with a boxcar of 5 pixels width.

By its nature, Stokes $V$ signals originating from the solar photosphere should be fitted by a dispersion profile with a Doppler core and extended collisional wings rather than by Gaussian functions which describe equilibrium processes. The dispersion profile together with its derivatives are given by Faraday-Voigt functions, whose evaluation using approximation formulae requires more time than the evaluation of a Gaussian function and its derivatives. The method described in this section is used for detection of the weakest $V$ signal with amplitudes at the level of the polarimetric sensitivity of the data $(<1 \%)$. Such signals are strongly influenced by the noise and smeared by the limited spatial resolution of the telescopes, and in case of the VTT data also by the transmission function of the 2D spectrometer. Thus, some of the information is irretrievably lost. The detection algorithm is intended only to find the location in the field of view of the weak $V$ signals and to estimate their amplitudes and separations. Moreover, in spite of the difference between dispersion and Gaussian profiles the chosen function gives a satisfactory fit even to relatively strong $V$ signals with amplitudes above noise, and which in principle do not need additional treatment (Fig. 2.14 upper row). Therefore, the choice of the analytical function for fitting seems to be reasonable.

The choice of the initial vector of parameters is important for the used gradientexpansion algorithm. For the fitting of noisy signal the method will converge only if 

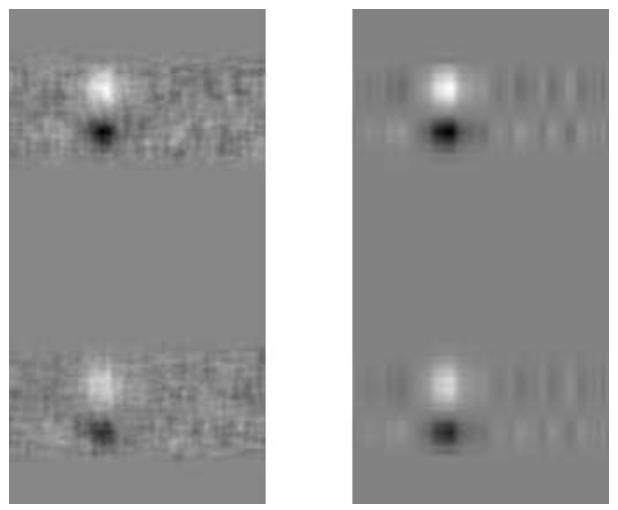

Figure 2.15: Example of the application of SVD to 1D spectrograms for image detection purposes. Left image: reduced Stokes $V$ spectrogram multiplied with a mask to remove noise in the areas with no $V$ signal and to avoid artifacts from further SVD; right image: first component of singular value decomposition of the left image detects the presence of Stokes $V$ signal with amplitudes below noise level.

the guess parameters of the fitting analytical function will be close to the sought ones. To estimate the guess parameters the singular value decomposition was once more applied to the reduced Stokes $V$ spectrograms in the same way is it was done for spatial filtering of 1D spectra, but now only the first component of the decomposition was retained (Fig. 2.15). As it was already mentioned, the SVD analysis is very efficient when applied to 1D spectra. It has completely removed noise and kept only the signal, which, however, cannot be considered as true $V$ profiles and used for further analyses, since all obtained profiles are symmetric and free of Doppler shifts. Due to the peculiarities of the SVD the first component carries only the most significant part of the signal, but not sufficient. The addition of more components will restore the neglected information but together with the noise. Therefore, the first component of the SVD is used only as the 1-st approximation to the sought signal and the detected $V$ profiles serve only for estimation of the amplitudes, polarities and location of the real signal. For the 2D spectra SVD was applied in the same but to the $\mathrm{Y} \lambda$ slices of the 3D data cube $I(x, y, \lambda)$ to emulate 1D spectrograms (Fig. 2.16). SVD detects very efficiently Stokes $V$ signal with amplitudes below the noise level even for data with higher level of noise and poorer spectral resolution and sampling.

In a such a way SVD provides guess values of the parameters which are used as an initial vector for the non-linear least squares fitting. But even starting with realistic guess values the gradient-expansion algorithm can converge to a set of free parameters which gives a non-Stokes $V$ looking function. To fix it an additional conditional block was implemented to the code. A set of distinguishing features which give a typical "portrait" of Stokes $V$ was selected. For example, the lobes of $V$ profile should have different signs, the distance between lobes should lie in a range typical for the spectral line, usually the order of the Doppler width of the line and bigger for stronger fields, the widths of the lobes and the zero crossing of the $V$ profile should be reasonable. The function returned by the fitting routine is compared with the defined "portrait". A function exhibiting an "appearance" different from Stokes $V$ is ignored and the whole algorithm starts from the beginning with the next profile in the spectrogram. 


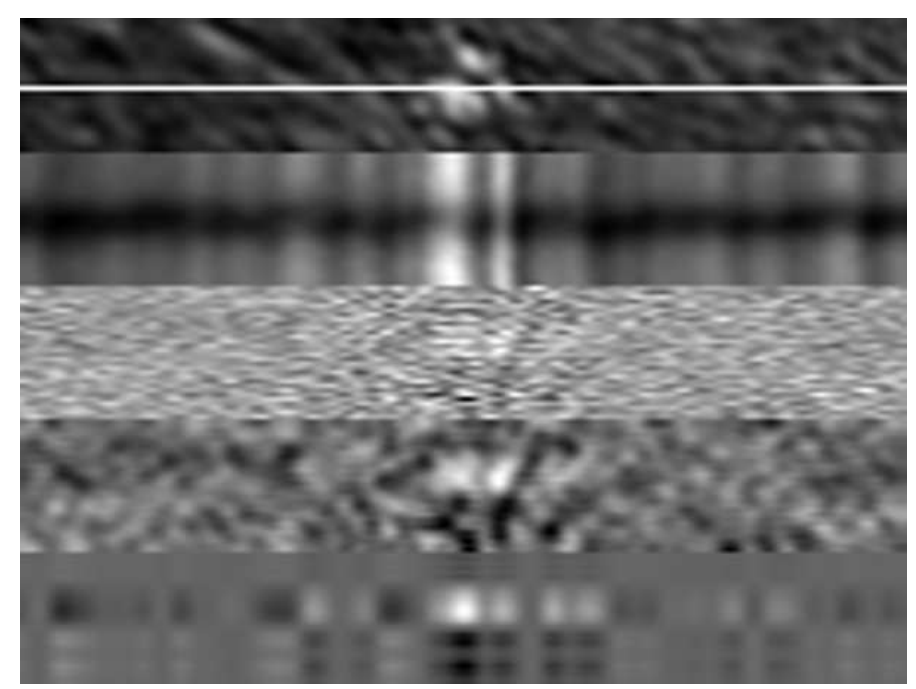

Figure 2.16: Application of the SVD for detection of weak Stokes $V$ signal in Y $\lambda$ slices of 2D spectrograms in the Fe II $6149.3 \AA$ line. From top to bottom: subfield of speckle reconstructed image showing the virtual slit for the $y$ - $\lambda$ slice; Stokes $I(y, \lambda)$ spectrogram from the 'slit'; original $V(y, \lambda)$ spectrogram from the 'slit'; boxcar smoothing of the original $V$ spectogram; SVD analysis of $V$ spectrogram retaining the largest singular value. The sizes in spatial and in wavelength directions are $26^{\prime \prime}$ and $480 \mathrm{~m} \AA$, respectively.
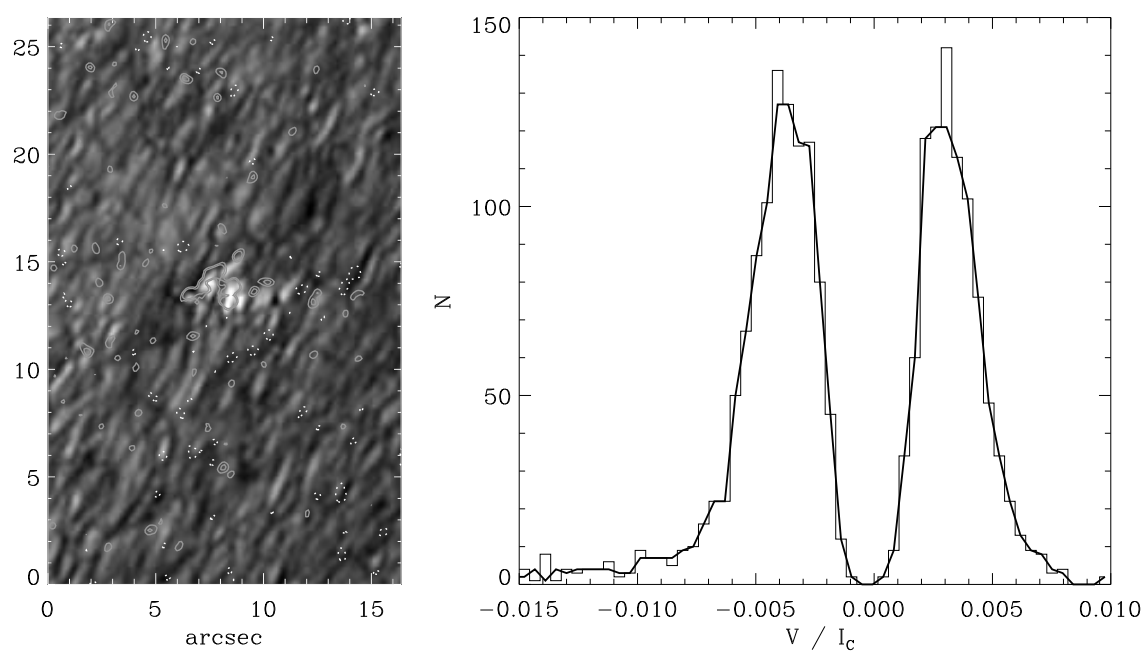

Figure 2.17: The result of Stokes $V$ recognition by means of the SVD. Left panel: Broadband reconstructed image overplotted with contours of magnetic signal. Solid and dashed contours denote different magnetic polaritites. Right panel: Distribution of Stokes $V$ amplitudes in units of the continuum intensity. 
To test the developed Stokes $V$ recognition algorithm the high spatial resolution 2D spectrogram observed with FPI-VTT in the Fe II $6149.3 \AA$ line was chosen because on the one hand the line is weaker and less magnetically sensitive (has smaller Landé factor) than the two other observed lines of neutral iron, resulting in small $V$ amplitudes and on the other hand the data were taken under best seeing conditions, which allowed to set the exposure time to $30 \mathrm{~ms}$, resulting in better SNR of the data.

The described algorithm was applied to the spectrogram and has recognized Stokes $V$ signals with absolute values in the range from 0.017 to 0.001 in units of the continuum intensity of the surrounding photosphere. The strongest $V$ signal $(\sim-0.015)$ was found, as expected, in faculae. Apart of it a multitude of magnetic patches of both polarities (Fig. 2.17 left panel) was detected. As it can be seen on the histogram (Fig. 2.17 right panel) the most frequent amplitude is 0.004 in both polarities, which are almost equally represented on the magnetogram with a small excess of the fields with polarity of the facula, which itself is represented in the histogram by an extended tail in the negative amplitudes of the distribution.
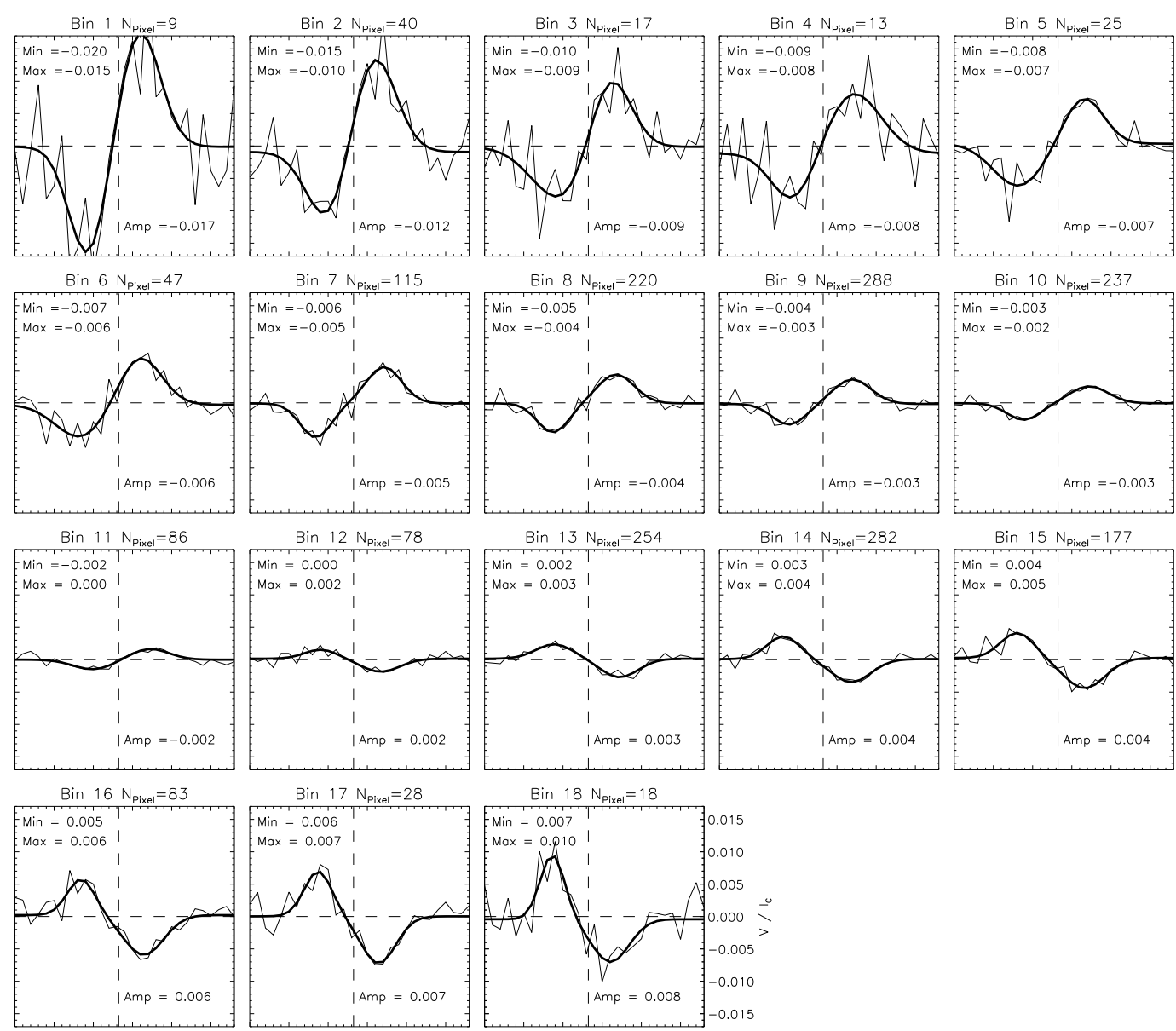

Bin $10 \mathrm{~N}_{\text {Pixel }}=237$
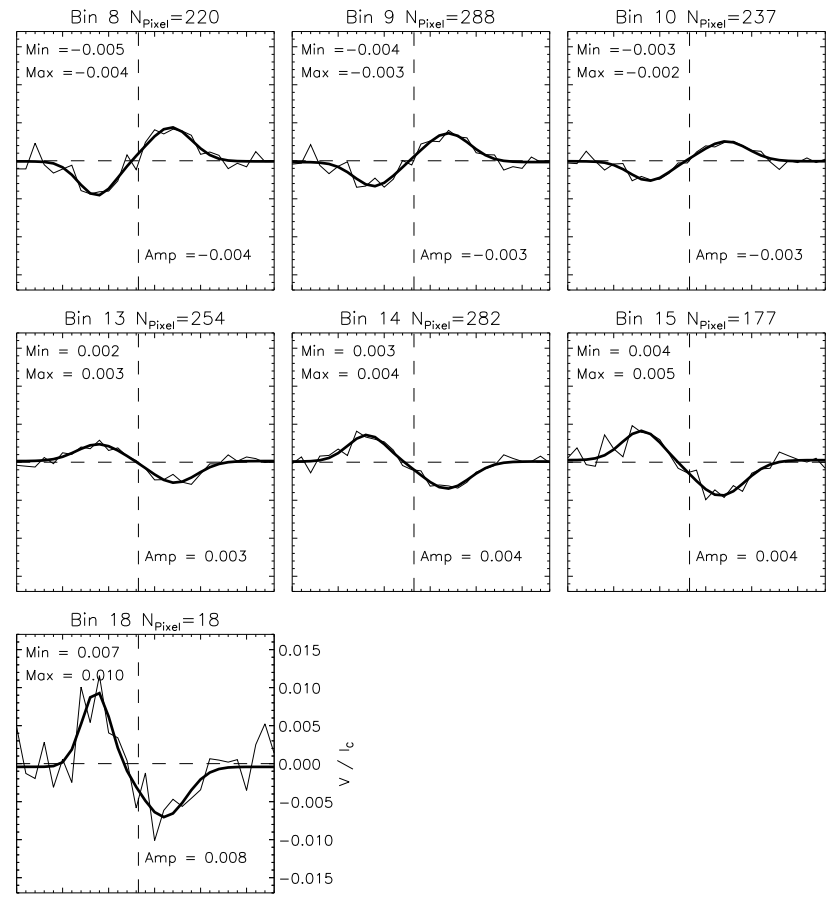

Figure 2.18: Stokes $V$ profiles (thin lines) averaged in bins overplotted with their fits (thick lines). All plots are scaled in the same way. The scae of the Y axis is given in the last plot. For each bin the interval of the amplitudes, the number of pixels in it, and the amplitude of the fitted $V$ profile are given. 
There still remains a question if all detected signals are reliable and how far can we trust the results of signal recognition. To test it we have collected all found $V$ signals in 19 amplitude bins (of unequal width) covering the whole range from the strongest signal of one polarity to the strongest signal of the opposite polarity. Afterwards, according to the defined amplitude intervals binary masks were created with units corresponding to the pixels on the spectrogram with found $V$ amplitudes in the range given by the current bin, and zeros elsewhere. For each bin the real observed profiles and not the detected and described by an analytical function were multiplied with the corresponding mask and averaged. If the observed noisy $V$ profiles are really modulated by weak signal and the recognition algorithm is sensitive enough to feel this modulation, then such averaging will suppress the noise and increase the statistical significance of the signal, and if the found $V$ signal is artificial then averaging will just reduce the noise and give no signal.

The result of such averaging in bins is presented in the Fig. 2.18. In each interval of amplitudes the averaging has revealed significant signal. All intervals of averaging contain a different number of pixels, resulting in diverse noise levels. The first bin, for example, has only 9 pixels and the average $V$ profile is still quite noisy but the signal itself is strong enough, and the resulting SNR is acceptable for further analyses.

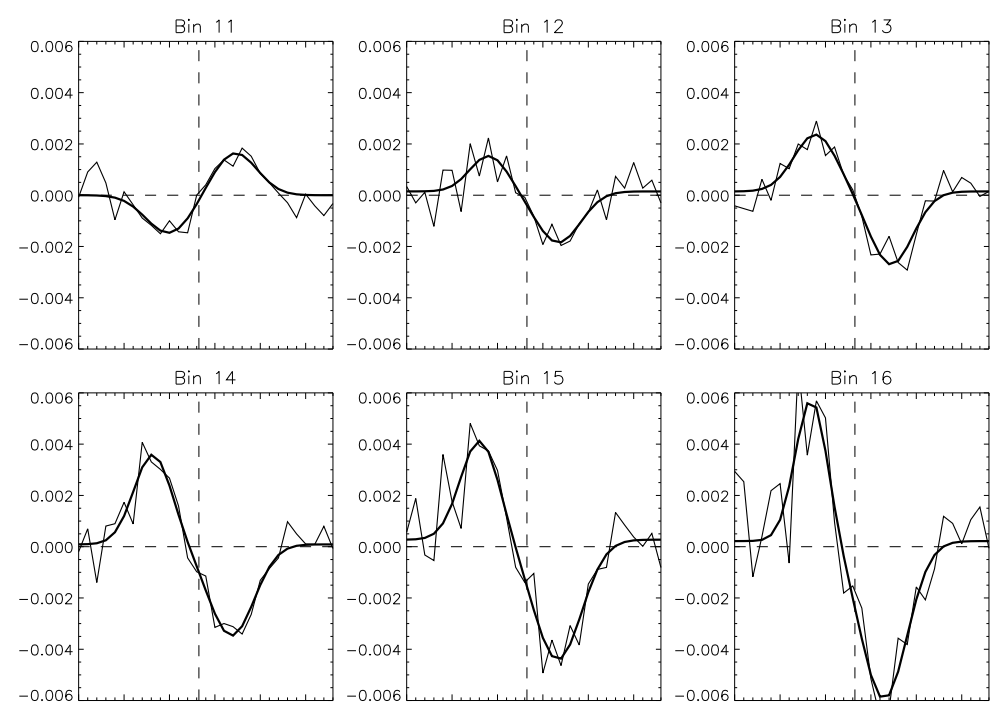

Figure 2.19: The lowest detectable Stokes $V$ signal.

But the most interesting are the profiles with the weakest signal (Fig. 2.19). For example, bin number 13 contains 254 pixels with recognized amplitudes in the range $0.002<V<0.003$. The pixel size of the data is $0.1^{\prime \prime}, 254$ pixels correspond to the area equal to the square with the side $\sqrt{0.1^{2} 254}=1.6^{\prime \prime}$. Thus, such selective averaging is equivalent to low resolution observations of magnetic fields occupying a larger area, which are characterized by high SNR. If one pixel has typically a noise level of $1 \%$ or 0.01 in units of the continuum intensity then averaging profiles from $N$ pixels will reduce the noise to $1 \% / \sqrt{N}$. For $250-280$ pixels (bins 13 and 14) the level of noise will be about $0.06 \%$ and accordingly the SNR $\sim 5$.

Thus, the developed signal recognition algorithm is indeed sensitive to the amplitudes much below the noise level, the found Stokes $V$ signal (including the weakest one) can 
be considered as reliable and the code can be used for the reduction of polarimetric data. Another important conclusion from this section is that high spatial resolution 2D spectrograms have a big potential of polarimetric sensitivity. From the example of our data it was shown that applying sophisticated methods of data reduction the polarimetric sensitivity can be improved by one order at least and comes to $10^{-3}$ of the continuum intensity.

\subsection{Instrumental polarization}

Radiation passing through an optical system of a telescope undergoes instrumental polarization or crosstalk effects. Due to numerous oblique reflections along the optical path the polarization state of radiation can be changed and the measured Stokes vector $\boldsymbol{I}^{\prime}$ will differ from the true one $\boldsymbol{I}$. Instrumental polarization is described mathematically as multiplication of the input Stokes vector with the Mueller matrix

$$
\left(\begin{array}{c}
I \prime \\
Q^{\prime} \\
U^{\prime} \\
V \prime
\end{array}\right)=\left(\begin{array}{llll}
m_{11} & m_{12} & m_{13} & m_{14} \\
m_{21} & m_{22} & m_{23} & m_{24} \\
m_{31} & m_{32} & m_{33} & m_{34} \\
m_{41} & m_{42} & m_{34} & m_{44}
\end{array}\right)\left(\begin{array}{c}
I \\
Q \\
U \\
V
\end{array}\right)
$$

where $m_{i k}$ are the elements of the Mueller matrix, which describes the polarization properties of the optical system.

The spectropolarimetric observations obtained for this study contain the $I$ and $V$ components of the Stokes vector. Therefore, in this section, we estimate only the $(Q, U) \rightarrow V$ crosstalk. The crosstalk from $(Q, U, V)$ to $I$ is very small, because of the weak amplitudes of the polarimetric signals from small-scale magnetic structures compared to the $I$ profiles. The measured Stokes $V$ profile has contributions from $Q$ and $U$

$$
V^{\prime}=m_{42} Q+m_{43} U+m_{44} V
$$

In this equation we do not account for the term $m_{41}$ because the $I \rightarrow V$ crosstalk was corrected during data reduction. Many spectrograms containing flat fields were averaged. Assuming that no polarization signal should remain after such averaging, the residual signal was considered to contain only instrumental effects including $I \rightarrow V$ crosstalk and it was subtracted from the individual $V$ profiles (Sect. 2.2.6).

Sánchez Almeida et al. (1991) have shown that the Gregory Coudé Telescope has low and slowly varying instrumental polarization. Therefore, we study here crosstalk effects only for the observations with the Vacuum Tower Telescope. The coelostat system of the VTT can produce instrumental polarization, which can not be ignored especially for the observations of polar faculae, which very likely possess strong magnetic fields and are observed close to the solar limb, where strong linear polarization is expected from inclined magnetic fields.

A measurement of the elements of the Mueller matrix for the VTT was made by Hofmann (2000). Capitani et al. (1989) have calculated values for the coelostat system in the Donati Solar Tower at Arcetri, which are changing with declination of the Sun and during the day. For our estimations of the crosstalk effects we have chosen average values: $m_{42}=0.05, m_{43}=0.3, m_{44}=0.9$. 
Due to its peculiar Zeeman pattern the transition Fe II 6149.3 produces no linearly polarized components and therefore does not exhibit $(Q, U) \rightarrow V$ crosstalk.

For the lines Fe I 6301.5, 6302.5 the effect of instrumental polarization was estimated using synthetic Stokes profiles, obtained from numerical simulations of faculae structures (Sect. 4 and Sect. 5). Simulations of the $(Q, U) \rightarrow V$ crosstalk were performed in the range of $\mu=0.4-0.1$ in accordance with the range where PFs were observed. Synthetic profiles were convolved with the Airy function of the FPI spectrometer in order to simulate the weakening of the intensity profiles due to the limited spectral resolution of the observations.

The $Q$ and $U$ components of the Stokes vector describe linearly polarized light in the planes rotated by certain angles relative to some reference direction. However, the synthetic $Q$ and $U$ profiles were calculated for the arbitrary choice of the reference directions, which do not necessarily coincide with the polarization planes of the optical devices for which elements $m_{42}$ and $m_{43}$ were defined. Therefore, we consider the worst case with the largest element of the Mueller matrix

$$
V^{\prime}=m_{43} \sqrt{Q^{2}+U^{2}}+m_{44} V
$$

The Figs. 2.20 and 2.21 present in the upper panels the comparison of the calculated Stokes $V$ profiles with $Q$ and $U$ at different heliocentric angles. In the lower panels the effect of contamination of $V$ signal with $Q$ and $U$ is presented.

It was found that at $\mu=0.4$ the amplitudes of the $Q$ and $U$ profiles are comparable with the $V$ signal, and therefore the contamination of the circularly polarized component with the linearly polarized radiation is acceptable. Since the blue and red lobes of the $Q$ and $U$ profiles are of the same sign they substantially change only the asymmetry of the $V$ profiles, while the separations and average amplitudes defined as a difference between maximum and minimum of the $V$ profile are less effected. Accordingly, calibration of magnetic field measurements from amplitudes and separations are justified.

Closer to the limb at $\mu=0.2$ the crosstalk becomes stronger due to the increased contribution from the $\sqrt{Q^{2}+U^{2}}$ signal. The $\lambda 6301.5$ line which is less sensitive to magnetic fields (produces weaker $V$ signal) suffers more from crosstalk at $\mu=0.2$ (Fig. 2.21). The asymmetry of the $V$ profile of the $\lambda 6302.5$ line is also strongly changed by the crosstalk. Yet, the averaged amplitude can be still correctly defined for this spectral line.

At the extreme limb, at $\mu=0.1$, observations of only the $V$ component with a coeleostat system are not efficient because of the strong crosstalk and because the $V$ amplitudes decrease below the detection limit. The latter is supported by our observations that no significant $V$ signal was found at the extreme limb.

We conclude that, although the crosstalk effects are strong in the data recorded with the VTT, the obtained observations are reliable because

- the worst case of crosstalk was estimated, the real contamination can be smaller;

- most of the data were recorded at $0.2<\mu \leq 0.4$;

- crosstalk substantially changes asymmetries of Stokes $V$, but not the separations and amplitudes, which were analyzed in this study;

- the main conclusions from the analyses of the VTT observations were compared with results from the GCT, which has lower instrumental polarization. 

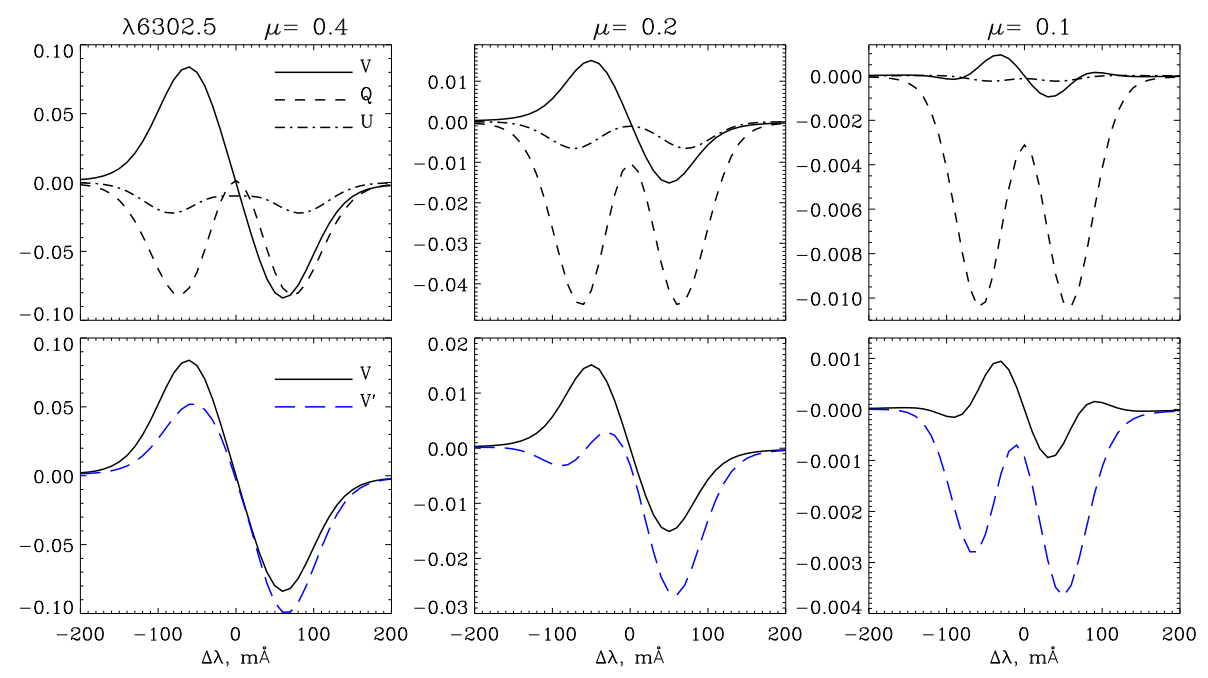

Figure 2.20: Upper panels: Stokes $Q, U$ and $V$ profiles of the $\lambda 6302.5$ line from synthetic faculae at different heliocentric angles. Lower panels: simulated $(Q, U) \rightarrow V$ cross-talk effects.
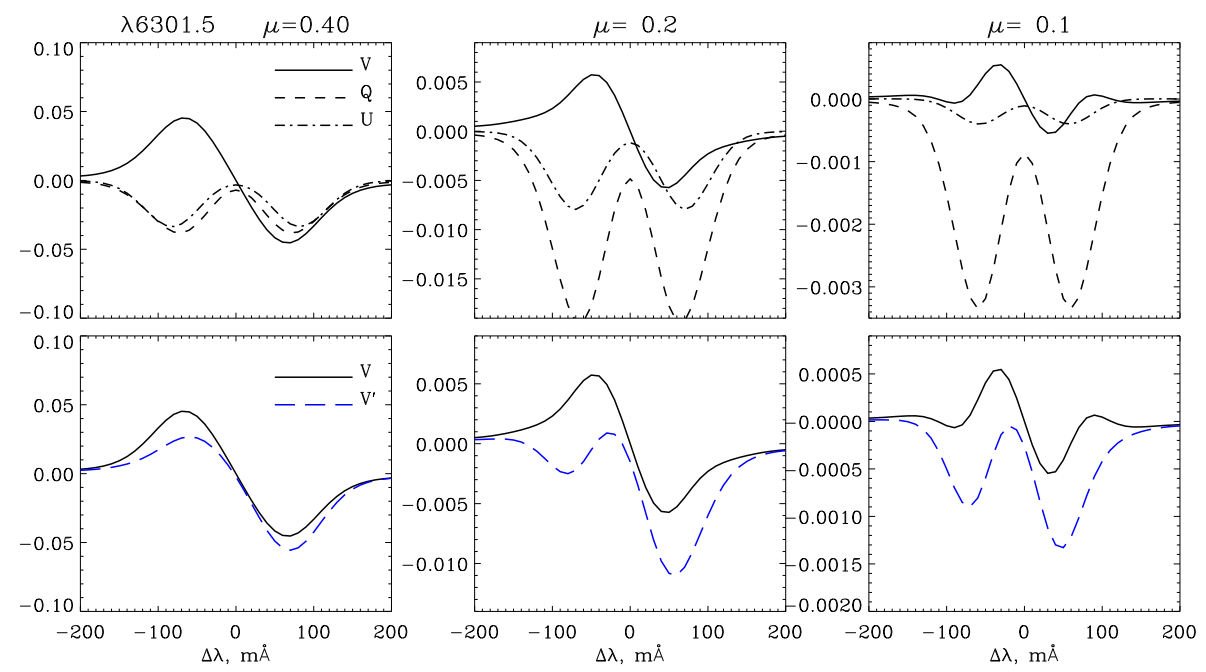

Figure 2.21: The same as Fig. 2.20 but for the line $\lambda 6301.5$

Finally, we note that for an efficient observational study of magnetic fields at the extreme limb of the Sun the full Stokes vector should be measured and observations should be performed with a telescope which combines high spectral and spatial resolution with a high spectropolarimetric sensitivity and low instrumental polarization. 



\section{Analysis of the observations}

In this chapter we present the study of the observational properties of polar faculae and give possible interpretations. A reliable determination of the photometric and spectropolarimetric characteristics of polar faculae is the key to understanding the phenomena as a whole. Thoroughly selected and reduced direct images and spectrograms obtained with both telescopes (GCT and VTT) were used to take the advantages of both instruments: high spectral quality of 1D spectrograms and high spatial resolution, provided by speckle interferometry.

\subsection{Photometric properties of polar faculae}

\subsubsection{Brightness contrast, center-to-limb variation}

The intensity distribution in PFs and the center-to-limb variation (CLV) of the brightness can provide the knowledge about the thermal structure of PFs, and can serve as an important observational constraint for further verification of numerical models.

The evident property of PFs is that they, as well as equatorial faculae observed in enhanced network, exhibit increased intensity contrast in continuum near the limb. The estimation of the true value of the brightness contrast of small-scale facular points is a very difficult task. The values which are given by observations vary in a big range. They obviously depend on many factors, such as: seeing conditions during the observations, resolution of the telescope, size of faculae, temporal state of the PF, etc.

To measure the brightness contrast in convenient units we introduce the relative brightness $\Delta I$, defined as the difference of the local intensity and the averaged one in percent of the average intensity:

$$
\Delta I=\frac{I-<I>}{<I>} \times 100
$$

where $I$ measures the absolute intensity, and $\langle I\rangle$ the averaged surface intensity approximated by a fitted paraboloid. A second order surface was chosen to remove intensity gradients across the field of view, caused by limb darkening near the limb. The above definition of $\Delta I$ will be used throughout this work.

As it was already mentioned the angular resolution of ground-based observations of the Sun, independently from the diameter of the entrance pupil, are usually limited by the seeing to typically $1^{\prime \prime}$. As a result, structures with sizes below this resolution will be unavoidably smeared, and the apparent contrast will be reduced. The image reconstruction improves the spatial resolution of the data, and as a result the contrast of small-scale 
bright elements increases dramatically. For example, the maximum contrast of the faculae on one of the best images observed with the VTT (Fig. 2.10) without applying restoration algorithms is about $30 \%$ relative to the quiet surrounding photosphere, it increases wth reconstruction to $50 \%$. This value was measured for a very big and bright cluster of facular points. Individual PFs were found to have even higher contrast with peak intensity reaching $80 \%$ ! More common values lie in the range of $20 \%-50 \%$, which are anyway very high compared to $3 \%-5 \%$ of intensity variation of the granulation near the limb. The measured intensity contrast also depends on the distance of the PF from the limb.

CLV. The center-to-limb variation of the intensity of faculae is one of the enigmas of small-scale structures in the solar atmosphere. Even on low resolution images observed at low latitudes we can see an increase of the brightness contrast towards the limb. On white-light images with low resolution $\left(>1^{\prime \prime}\right)$, from the variety of photospheric magnetic fields, only sunspots and pores are apparent at the center of the solar disk. High resolution observations allow to detect more objects probably associated with magnetic fields, such as abnormal granulation and isolated facular points, or bright points, which are best detected in the $\mathrm{G}$ band ( $\mathrm{CH}$ molecular band near $4310 \AA$ ), where they have increased contrast (Muller \& Roudier 1984; Berger et al. 1998). By means of spectroscopic observations more magnetic phenomena become visible at the center, for example line gaps in magnetic knots (e.g. Kneer \& von Uexküll 1991; Soltau 1997).

At larger heliocentric angles faculae in enhanced network become visible even with low resolution in continuum. Contrasts of faculae, which are mainly located in the active region belt, have been measured by Lawrence \& Chapman (1988) with a spatial resolution of approximately $2^{\prime \prime}$ at $\lambda=6264 \AA$. They obtain contrasts in the range of $3-8 \%$ for $\mu<0.5$. Auffret \& Muller (1991), from high-resolution (0".23) photographic observations at $\lambda=5750 \AA$ near the Sun's equator, presented the center-to-limb variation of network bright points with a maximum contrast of up to $40 \%$ at $\mu \approx 0.4$. These authors note that the most significant CLV is obtained only for bright points of medium size of 0."4-0.7. Ortiz et al. (2002) analyzed MDI data at $\lambda=6768 \AA$ with moderate spatial resolution. For strong magnetic fields they find contrasts of up to $6 \%$ with a maximum indicated near $\mu=0.3$. Sánchez Cuberes et al. (2002, see also references therein) measured contrasts with the Swedish Vacuum Solar Telescope on La Palma at wavelengths of $0.8 \mu \mathrm{m}$ and $1.55 \mu \mathrm{m}$ and obtain for the former wavelength a maximum contrast of $4-8 \%$ at $\mu=0.3$.

Observations of polar faculae at the disk center are in principle possible but at the moment not available. They would be accessible only by means of a space-born telescope located somewhere above a solar pole and not in the plane of the ecliptic. Therefore our study is limited to large heliocentric angles only. Observations which were taken into account cover the range of $\mu=\cos \theta$ from 0.4 to 0.15 .

All data used in this work were obtained in the years 2001-2002. These were the years close to the maximum of solar activity. The Sun exhibited strong activity at low latitudes (high sunspot number), and accordingly low polar activity. The epoch of the polarity reversal finished by the end of 2000, beginning of 2001. The following years 2001 and 2002 were the first years of the new polar activity cycle, the time when PFs are observed relatively rarely. Therefore, to study the CLV of the continuum contrast of PF we have used the observations taken with both telescopes to improve the statistical sample. 


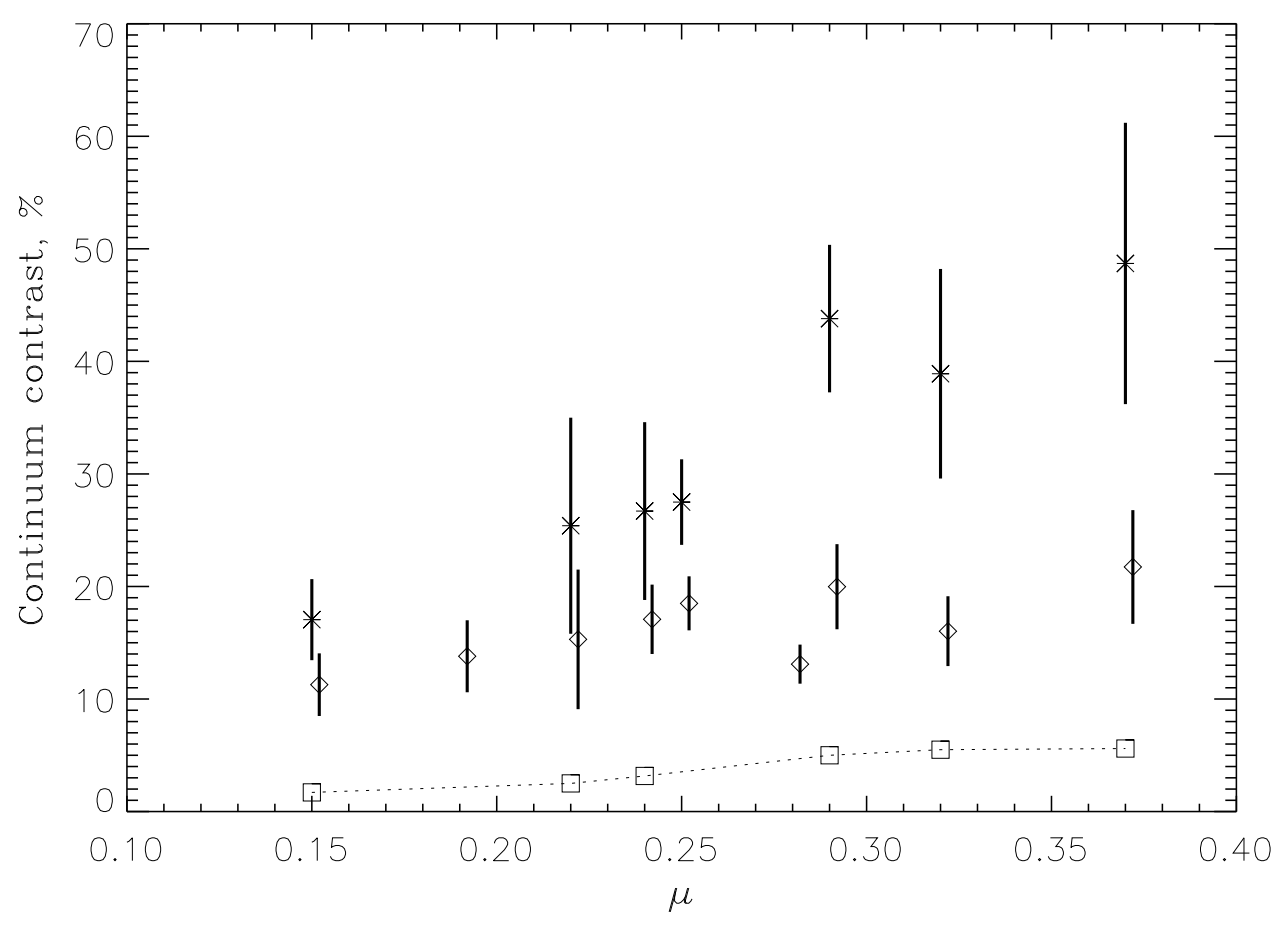

Figure 3.1: Center-to-limb variation of the polar faculae continuum contrast in a limited range of $\mu=\cos \theta$. Diamonds: moderate spatial resolution observations (1", GCT data and VTT images without speckle reconstruction), asterisks: high resolution observations $(0 \prime 2$, broadband speckle reconstructed images), squares: granular contrast of the surrounding quiet photosphere. Vertical bars denote one $1 \sigma$ variation of the measured values due to changing seeing conditions, time evolution, etc.

The CLV of faculae continuum contrast was measured in the range of $\mu$ available for observations and is presented in Fig. 3.1. To separate the effect of limited spatial resolution on the apparent contrast all observations were separated into two groups. GCT observations and VTT broadband images before speckle reconstruction form the first group (diamonds in Fig. 3.1) with resolution of about 1", sometimes better. In 1D spectrograms (CGT) the contrast was measured in the continuum near spectral lines. The second group (asterisks in Fig. 3.1) includes reconstructed broadband frames (VTT only) and is characterized by spatial resolution close to the diffraction limit of $0.2^{\prime \prime}$. The CLV for both groups is plotted separately. We summarize the main peculiarities of the CLV of PFs:

- The intensity contrast of PFs decreases towards the extreme limb. Observations with high resolution: from $50 \%$ at $\mu=0.4$ to $15 \%-20 \%$ at $\mu=0.15$, with moderate resolution: from $20 \%$ at $\mu=0.4$ to $10 \%$ at $\mu=0.15$.

- The maximum of the contrast is at $\mu \geq 0.4$.

- The difference between intensity contrast from high and moderate resolution data is bigger for brighter structures observed at larger $\mu$. 
Two mechanisms were suggested to explain the CLV of faculae at low latitudes: the hot wall model (Spruit 1976, Deinzer et al. 1984b) and the hot cloud model (Knölker, Schüssler \& Weisshaar 1988).

Hot wall model: magnetic flux tubes are cooler than the surrounding. At the center of the solar disk a ray along the line-of-sight stays always inside the flux tube, never reaching hot layers as in photosphere where convection is not inhibited by flux the tube's magnetic field. At larger heliocentric angles rays from the hot walls can penetrate the flux tube towards the observer.

Hot cloud model: the plasma inside flux tubes is depressed due to the presence of a strong magnetic field such that the internal gas pressure plus magnetic pressure balance the external pressure.

$$
p_{g}^{i n t}+p_{m}=p_{g}^{e x t}
$$

In the medium with strong horizontal pressure gradients the effects of horizontal radiative transfer start to play the role. Fabiani Bendicho et al. (1992) have shown by means of $2 \mathrm{D}$ numerical radiative transfer calculations that radiation channeling leads to the enhancement of the temperature of the upper layers. At certain $\mu$ these heights become visible in white light resulting in an increased brightness contrast of faculae near the limb.

Both mechanism can be used for interpretation of the increased contrast of PFs near the limb. But not only the temperature structure of faculae can be responsible for the observed photometric properties. The partial evacuation of magnetic flux tubes which constitute faculae should also be taken into account. Magnetic elements are characterized by reduced gas pressure and accordingly low mass density (depending on the temperature). The deficit of the gas pressure inside flux tubes is compensated by the magnetic pressure. Stronger magnetic fields will cause stronger evacuation of the flux tubes, lower mass density (less absorbers along the line-of-sight) and higher transparency of the plasma in a magnetic element. A large number of transparent flux tubes on the ray along the lineof-sight would make deeper and hence hotter layers of the photosphere visible. Thus, not only the thermal structure of faculae, but also the magnetic field strength, the size and the number of magnetic flux tubes along the line-of-sight should be taken into account. An estimation of the contribution of each of these factors is possible only by means of numerical modeling.

\subsubsection{Fine structure and size}

On low-resolution intensity images PFs appear as bright diffuse structures with typical sizes of few arcsec. To study the fine structure of the phenomena under consideration and to obtain a more realistic estimation of the true size of the faculae, or of structures which constitute faculae, one will need the highest possible spatial resolution. For the current study we have selected the best quality broadband images observed with the VTT. Speckle reconstruction of such frames provides images with spatial resolution of 0 "' 2 close to the diffraction limit of the telescope (about $150 \mathrm{~km}$ on the Sun). Selected images (Fig. 3.2) were taken from different days and from different positions on the disk.

PFs appear to have a complex fine structure. Faculae which are visible on low/moderate resolution images as bright formations without internal structure, split up on reconstructed images into a number of smaller bright elements of subarcsec sizes, which are organized 

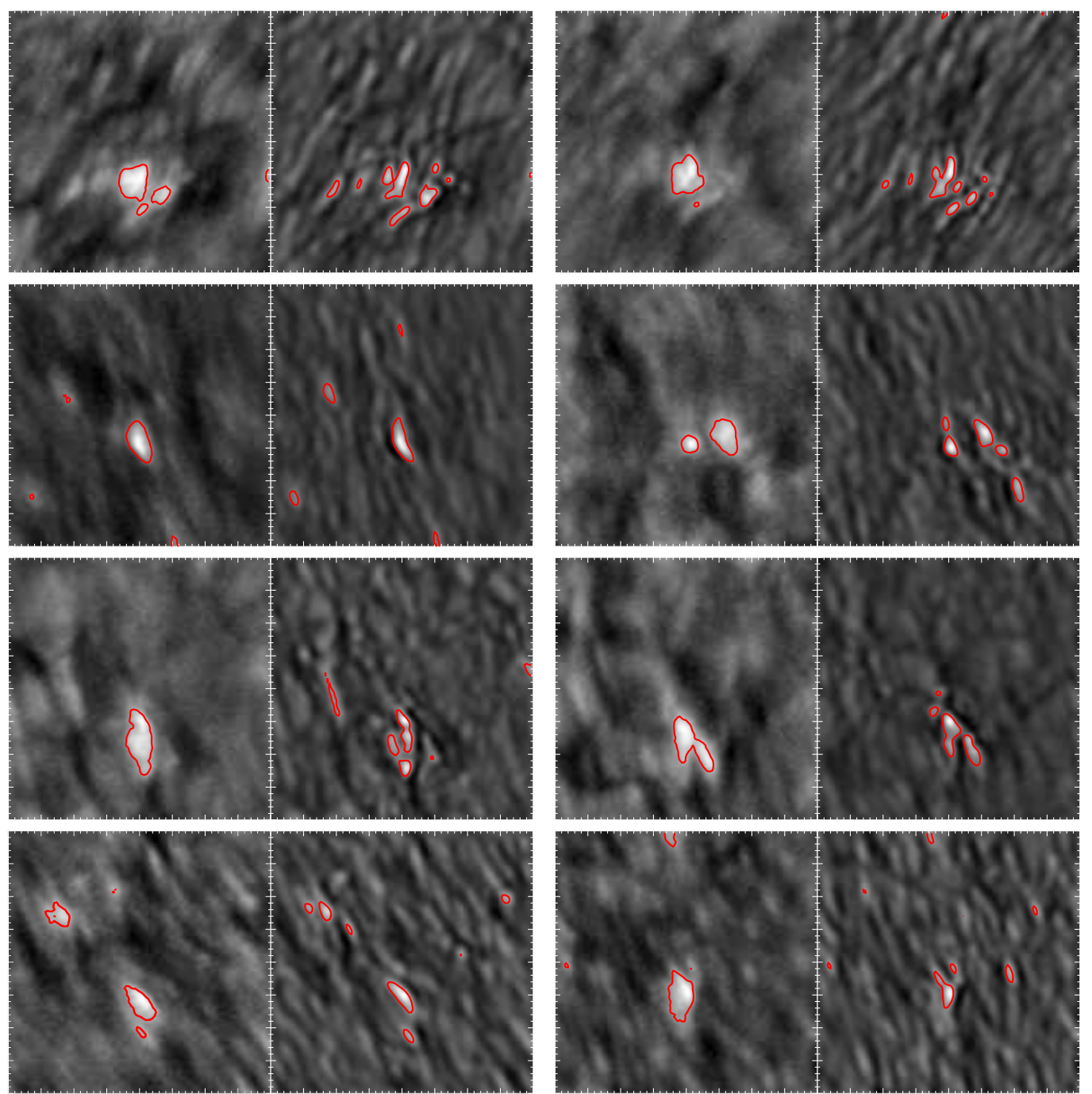

$\begin{array}{llllllllll}0 & 1 & 2 & 3 & 4 & 5 & 6 & 7 & 8\end{array}$

Figure 3.2: Fine structure of polar faculae. In each pair the left image corresponds to an observed broadband image and the right to the reconstructed. The size of each image is $8^{\prime \prime} \times 8^{\prime \prime}$. Overplotted contours outline areas with $\Delta I>3 \sigma_{\text {gran }}$, where $\sigma_{\text {gran }}$ is an RMS brightness contrast of the surrounding granulation.

in clusters or chains stretched parallel to the limb. On both raw and reconstructed images PFs and small scale facular points differ in sizes in directions parallel and perpendicular to the limb. Most of the structures are elongated along the limb, similar to other photospheric structures observed near the limb (granulation, sunspots), which exhibit perspective foreshortening. The principal difference is that small-scale magnetic flux tubes which constitute PFs are optically thin and near the limb the line-of-sight is oblique to the vertical direction and the ray crosses several flux tubes at different heights, therefore the contribution to the emergent intensity is very complicated. For the interpretation of the sizes of brightenings in the direction perpendicular to the limb we should consider 1) the vertical extent of flux tubes and 2) contributions to the ray from several inhomogeneities along the line-of-sight. 
The size of PFs in the direction parallel to the limb is easier to interpret. If we accept the concept of PFs as a concentration of MFTs then the brightest areas in a PF will correspond to the rays with the maximum contribution of individual MFTs along the lineof-sight to the emergent intensity, and hence the distance between the center of gravity (henceforth COG) of the brightenings will give an idea about the "lattice spacing", the average distance between single MFTs in the array of tubes. This value can be estimated, though it depends on the shape of the PFs and their internal organization. For regular necklace-like PFs the distance between "pearls" was found to be about 0".5 (or $300-$ $400 \mathrm{~km}$ on the Sun).

A final remark should be made in this section. It seems that even in the diffraction limited images we do not fully resolve the fine structures. Examining reconstructed images we found very bright isolated points (on a dark background) of a very small size and with regular circular shape. But as it was just mentioned the structures near the limb are usually stretched along the limb. A circular shape can be produced by structures with size much below the resolution limit convolved with the PSF of the telescope. So, probably, what we see is an Airy disk of the telescope, while photospheric magnetic structures consist of very small and bright structures.

\subsection{Time evolution}

All former studies of the temporal evolution of faculae are usually limited to the estimation of the lifetime of faculae, the time during which the structures are visible on the disk. Such estimations vary in a wide range, but it is established that PFs are generally long-lived structures, which can be visible on the surface of the Sun for hours. But still very little is known about the dynamics of the fine structure of PF. Such studies pose very high requirements on the quality of observations. If for the reliable determination of the intensity distribution and sizes of PF we need high resolution and good seeing, then for the study of the dynamics of the phenomena it is important that seeing conditions are good and stable during a long period of time. Seeing does not only change the intensity contrast, smoothing structures, but also introduces various geometrical distortions. Speckle reconstruction corrects the distortions of the image and makes it possible to study dynamics at small scale.

In order to construct time series we have used sequences of broadband images, observed with the VTT on 15 June, 2001, one week before summer solstice. It was a day with a good and, what is more important for the studies of the temporal behavior, enough stable seeing conditions. Two areas near the south pole $\left(\phi \sim-70^{\circ}\right)$ containing PFs were observed. At the first position (at $\mu=0.32$ ) a time series of 20 scans with 49 s cadence was obtained. So the duration of the sequence is 16.5 minutes. The second area $(\mu=0.29)$ with a PF was observed during longer time. Two time sequences with 20 scans each were recorded successively without changing pointing of the telescope, with a few minutes time gap between the end of the first sequence and the start of the second. So, the duration of the whole times series, the time between the first image in the first sequence and the last image in the second was about 37 min with a 4 min gap in between.

The observations of both PFs were thoroughly reduced and two short continuous movies were created (http://www.uni-sw.gwdg.de/ olok/pf), on which the evolution of 
the fine structure of the observed polar faculae become directly visible at high spatial resolution (Fig. 3.3). The reduction of the time series included the following steps:

- correction of each single image in a time sequence for atmospheric distortions with speckle technique,

- correction for a global shift between neighboring images by means of cross-correlation,

- in order to compensate for the time gap between the above two successive sequences additional images were obtained by the interpolation of the intensity in each pixel between the last frame in first series and the first frame in the second,

- the resulting $3 \mathrm{D}$ data cubes $I(x, y, t)$ were filtered in $k-\omega$ space, using a cone filter, in order to remove solar intensity oscillation and brightness flickering from image to image due to incomplete image reconstruction.

The whole picture of dynamics including granular convection near the limb is very different from the one seen at the center of the solar disk, where we see strictly vertical motions (upflows and downflows), and can trace horizontal displacements. In our case, close to the limb, at $\mu \sim 0.3\left(\theta=72^{\circ}\right)$ the situation is almost opposite, the horizontal flows in the photosphere are seen at an acute angle, almost parallel to the LOS, while we see the vertical motions almost from the side.

The temporal behavior of PFs is very different from the convective pattern. When observing faculae with low resolution we do not see much difference between the first and the last images in the time sequence. They have the same location and approximately same size and contrast, only slightly differing in shape. But when observed with high spatial resolution polar faculae appear to be highly dynamical structures. The fine structure of a PF in the first and last images is completely different. Tiny and bright facular points which constitute a PF are permanently evolving, they move, merge and split, decay and flare up again. All these changes occur on very short time scales, the picture can change noticeably even between two adjacent images, with time interval of only $49 \mathrm{~s}$.

Such a behavior probably reflects the interaction between the convective motions and individual thin MFTs. Horizontal flows can shake, bend and displace individual flux tubes. Moreover, magnetic tubes can be squeezed by the external pressure, which will change the intensity of the magnetic field and, accordingly, effect the optical thickness of the flux tubes. All this can change the contribution of the facular atmosphere to the emergent intensity, and in such a way change the observed intensity pattern.

The similar behavior is expected for network magnetic fields which are formed by horizontal flows which sweep together weak magnetic fields frozen into the plasma, producing concentrations of strong fields. Such a scenario is predicted by theory (Deinzer et al. 1984a, 1984b) and is in agreement with numerical MHD simulations (e.g. Vögler \& Schüssler 2003) of magnetoconvection and with observations which confirm that magnetic elements are concentrated in downflow regions (Koschinsky et al. 2001).

Polar faculae belong to another class of solar magnetic fields. Presumably, they are a part of the global magnetic field of the Sun and emerge from the photosphere already organized in bundles of magnetic flux tubes. So, such highly dynamical behavior of the phenomena should be associated not with the processes of the formation of faculae but with their evolution, the interplay between convection and global magnetic field at the level of the photosphere. 

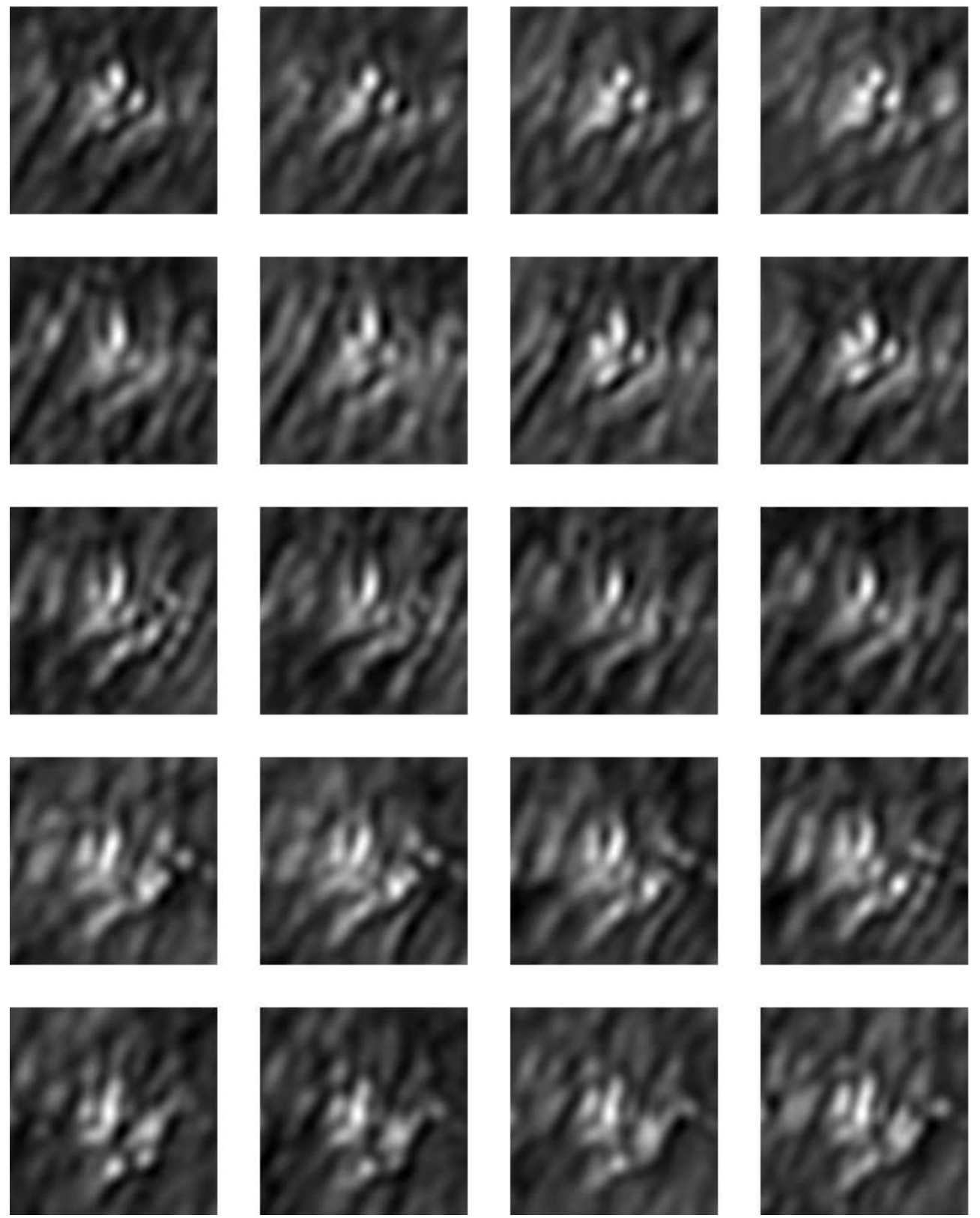

Figure 3.3: Evolution of a polar facula during 16 minutes. The sequence runs row by row from left to right and from top to bottom. The time step is $49 \mathrm{~s}$. The size of the FOV is $4^{\prime \prime} \times 4^{\prime \prime}$. 


\subsection{Spectropolarimetric properties of polar faculae}

Spectroscopic observations are potentially more informative than photometry, since they allow to study the phenomena at different heights in the atmosphere, and additionally to measure LOS velocities and magnetic fields. However, spectral information is more difficult to interpret because of the complicated mechanisms of spectral line formation in an inhomogeneous medium, and because of different processes contributing to the continuum and line core intensities. The goal of the study was surely to determine spectral properties of the radiation emerging from PFs, to estimate from the latter more properties of faculae and to establish several distinguishing characteristics peculiar to PFs, which together with the photometric properties will give a portrait of the typical PF.

Spectropolarimetric data in magnetic sensitive iron lines Fe I $6302.5 \AA$ (g=2.5), Fe I $6301.5 \AA(\mathrm{g}=1.67)$ and Fe II $6149.3 \AA$ (g=1.33) were obtained with high spectral and spatial resolution, carefully reduced and analyzed. All used spectral lines form in different layers of the photosphere below the temperature minimum in conditions close to LTE. The weakest line (of ionized iron) Fe II 6149.3 $\AA$ originates from the lowest levels in the atmosphere $(h \sim 100-200 \mathrm{~km})$, the neutral iron lines Fe I $6302.5 \AA$ and Fe I $6301.5 \AA$ form in upper layers, $h \sim 300-400 \mathrm{~km}$ and $h \sim 400-500 \mathrm{~km}$, respectively.

\subsubsection{Line core intensities and line depressions}

Intensity profiles observed in PFs were compared with the averaged photospheric profiles (Fig. 3.4 and Fig. 3.5). In all three lines the intensity in the line core in PFs is higher than in the surrounding photosphere, similar to the line gaps observed at the center of the solar disk (Kneer \& von Uexküll 1991).

Normalizing the Stokes $I$ profiles to their local continuum and comparing them again with the average photospheric profiles we study depressions of the spectral lines (Figs. 3.4 and 3.5 middle frames). It was found, that the line depression exhibits different behavior for all three lines under study. We summarize the properties of the intensity profiles from polar faculae:

- strong contrast both in the continuum and line core for all three lines;

- in the Fe II 6149.3 line the Stokes $I$ profiles from PFs $\left(I_{\mathrm{PF}}\right)$ are substantially stronger and broader than the averaged intensity profile from the surrounding quiet Sun photosphere $I_{\mathrm{QS}}$;

- in the Fe I 6301.5 line $I_{\mathrm{PF}}$ show almost the same line depression as $I_{\mathrm{QS}}$ but appear to be slightly broader than $I_{\mathrm{QS}}$;

- in the Fe I 6302.5 line $I_{\mathrm{PF}}$ are slightly weaker and broader than $I_{\mathrm{QS}}$.

Roughly speaking, the spectral line depression reflects the temperature difference between layers of continuum and line core formation. When a specific line is weak it originates from an atmosphere with small temperature gradients. When the same line is strong the atmosphere possesses a larger $T$ gradient. In terms of thermal structure of PFs we can suppose that the "hot cloud" model is preferred. Indeed, the temperature excess in upper layers will cause stronger ionization and could in principle explain the weakening 

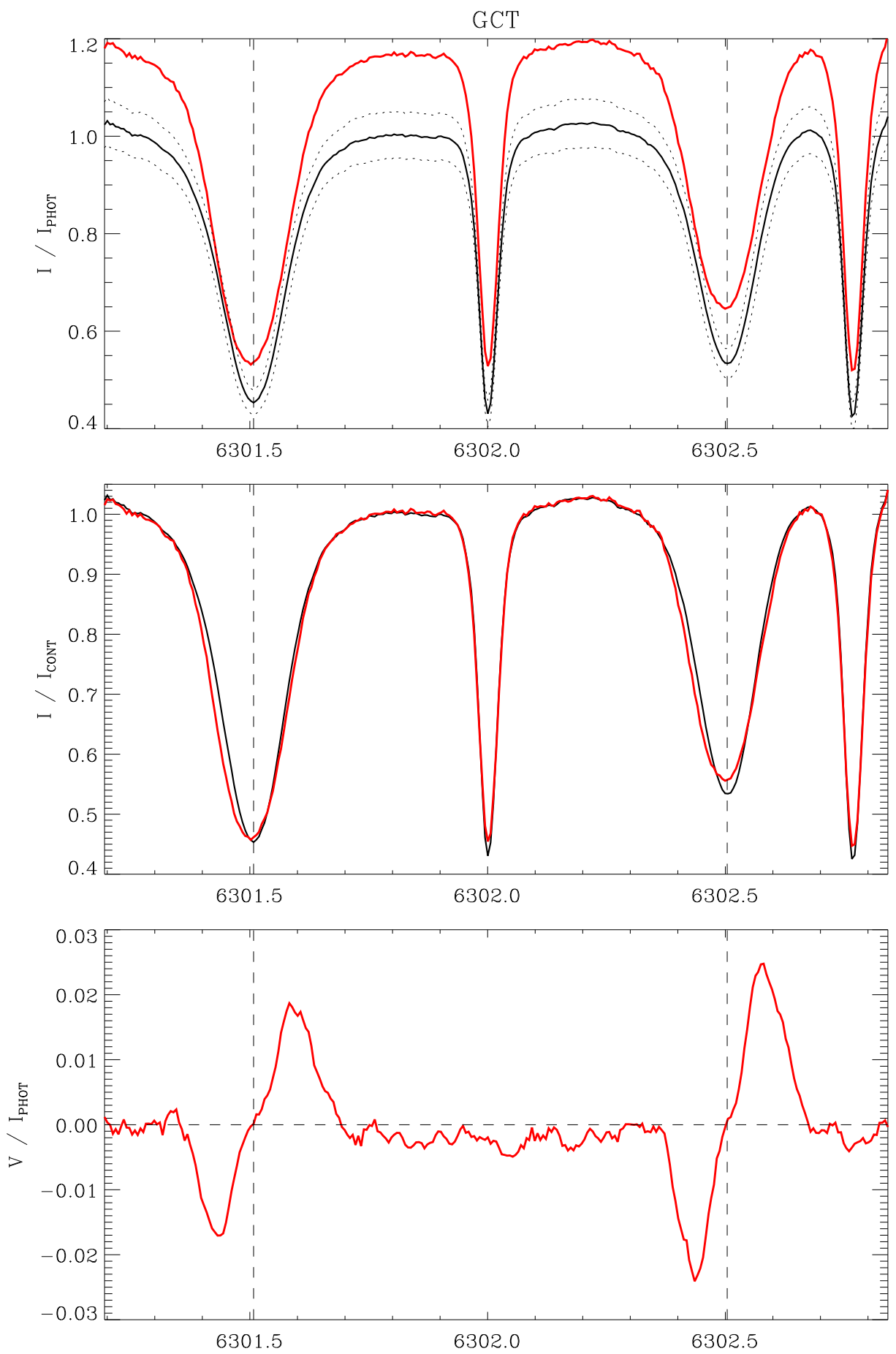

Figure 3.4: High resolution profiles in Fe I 6302.5, 6301.5 ^ observed with the GCT. Red thick lines refer to polar faculae, black thin lines to averaged photospheric spectra. Upper panel: Stokes $I$ profiles normalized to the photospheric continuum near $6301.8 \AA$. Dotted lines show $1 \sigma$ fluctuation of the intensity in the surrounding photosphere at different wavelength positions. Middle panel: Intensity profiles from a PF and from the average photosphere normalized to their local continuum. Lower panel: Stokes $V$ spectra observed in a PF, also normalized to the photospheric continuum. 

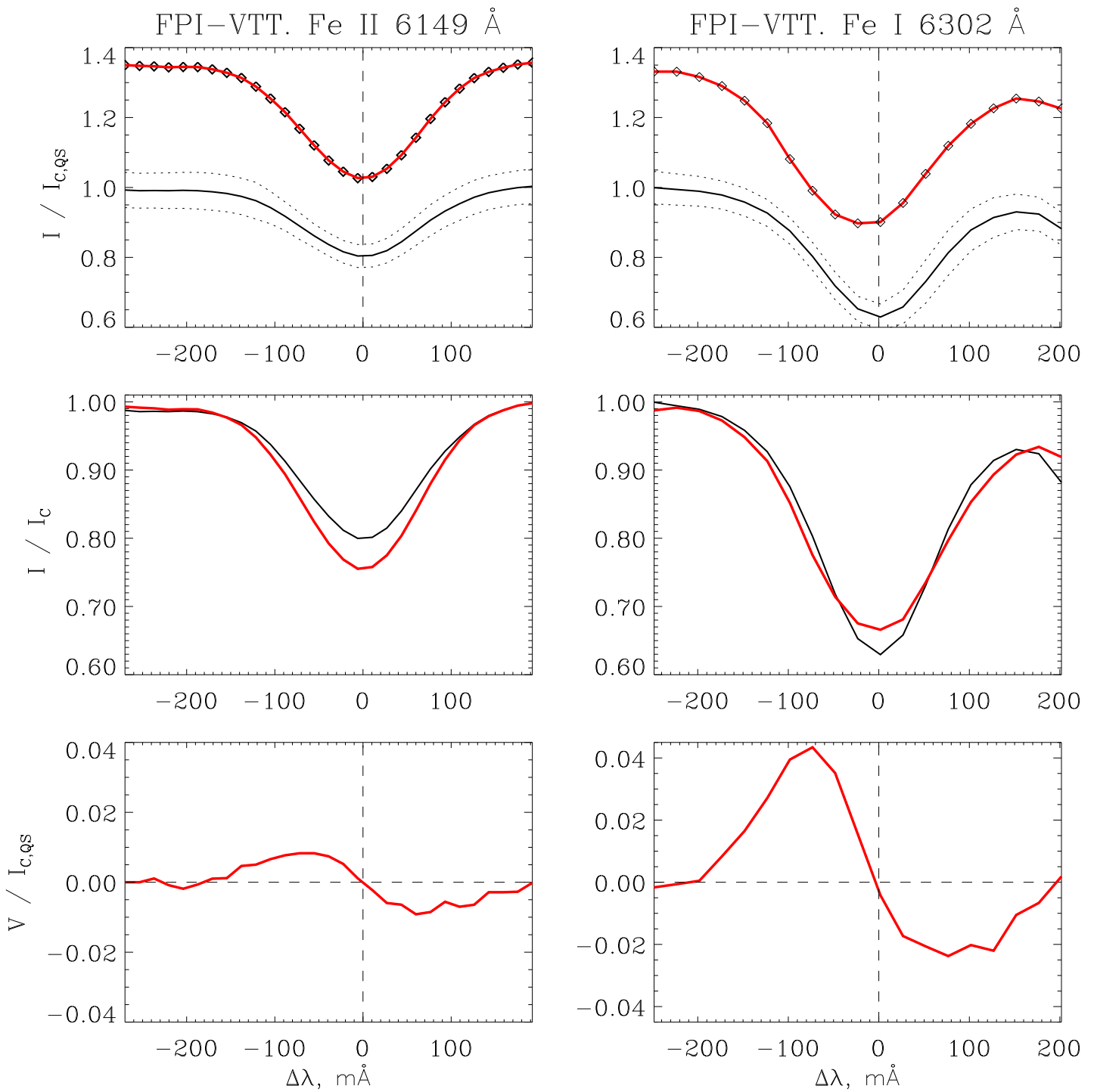

Figure 3.5: The same as in Fig. 3.4 but FPI-VTT observations with high spatial resolution in the Fe II $6149.3 \AA$ and Fe I $6302.5 \AA$ spectral lines.

of neutral iron lines, strengthening of the ionized line and broadening of all of them compared to the photospheric lines. But if we consider the influence of reduced gas pressure, which effects the optical thickness of the flux tube, and also consider the highly intermittent structure of the atmosphere along the line-of-sight the interpretation will not be straightforward.

The intensity profiles discussed in this section and presented in Figs. 3.4 and 3.5 correspond to the brightest pixels from the center of PFs. They aim at presenting typical line profiles from polar faculae, which are strongly effected by magnetic atmospheres. However, high spatial resolution of the spectropolarimetric data allows to study intensity profiles from other locations in PFs with smaller contribution from facula atmospheres. Thus, noticeable variations of spectral properties along the direction center-limb were found. We note, that Stokes $I$ profiles from the center side of PF are broader than from the limb side. 


\subsubsection{Velocities in polar faculae}

Our spectroscopic data allow the determination of the line-of-sight (LOS) velocities from Doppler shifts and asymmetries of spectral lines. For this, the spectra were additionally smoothed in wavelength direction with a 3 point boxcar. Then part of the spectral line near the core was interpolated with a 4th order polynomial, and the displacement of the line center was estimated. To calibrate Dopplergrams the shifts were measured relative to the position of the center of the line profile averaged over the whole field of view. In such a way $2 \mathrm{D}$ velocity maps with high spatial resolution were calculated and some of them are presented in Fig. 3.6 together with continuum and line core images.

The difficulty of the interpretation of the LOS velocity measured near the limb is in a contribution of horizontal flows in the photosphere to the line shifts. Apart of this, horizontal inhomogeneities of the photosphere make the medium along the LOS near the limb strongly intermittent. At the center of the solar disk the LOS coincides with the vertical direction and the rays along the LOS always stay within one type of convective element (upflow or downflow). At large heliocentric angles a ray along the LOS crosses several horizontal inhomogeneties so that the LOS velocity $v_{l o s}$ is very irregular and can even change its sign several times. If a spectral line is formed in the range of heights of $0-400 \mathrm{~km}(\lambda 6302.5 \AA)$, then the photosphere with horizontal extent of $\Delta x=H \tan \theta$ will contribute to the emergent intensity. At $\mu=0.2$ we have $\tan \theta=4.7$ which yields $\Delta x=1800 \mathrm{~km}(\sim 2.5)$. This horizontal extent can contain several convective elements with different velocity structure.

In Fig. 3.6 wee see that PFs are mainly located in regions with negative velocities (directed towards the observer). To interpret this result we need to separate the measurement from the above mentioned contributions of horizontal structures and flows. Some simple statistics on velocities measured in PFs at different positions from the limb were collected and compared with the CLV of the granular convective velocities. Scatter plots presenting the relations between continuum intensities and LOS velocities measured in PFs and the ambient photosphere are presented in Fig. 3.7. At quiet Sun disk center we see pure convective behavior (strong correlations between intensity and velocity), off the center ( $\mu \sim 0.4$ and $\mu \sim 0.3$ ) the intensity contrast and the velocities become smaller, but the projection of the vertical flows on the LOS is still bigger than the projection of the horizontal motions, therefore the correlation is still significant. Closer to the limb $(\mu \sim 0.2)$ the correlation between $\Delta I$ and $v$ almost disappears due to the increased contribution of horizontal structures and flows, the intensity contrast and $v_{l o s}$ are small here. Polar faculae which are represented in Fig. 3.7 by triangles exhibit a behavior different from the convective pattern. They possess strong intensity contrasts near the limb and were found to be located always in regions with negative LOS velocities. This result is obtained in all observational data without any exception, though within a limited statistical sample.

Stokes $I$ profiles are characterized by good signal-to-noise ratio, which allows accurate measurements of the LOS velocities from line core shifts. However, these measurements must be interpreted with care. Due to the limited spatial resolution of observations Stokes $I$ profiles emerging from PFs are contaminated with signals from the non-magnetic photosphere in a resolution element. Therefore, basing only on the Stokes $I$ analyses it is impossible to distinguish between signals emerging from a facula atmosphere and from the field free components around and behind (along the LOS) the facula element. 

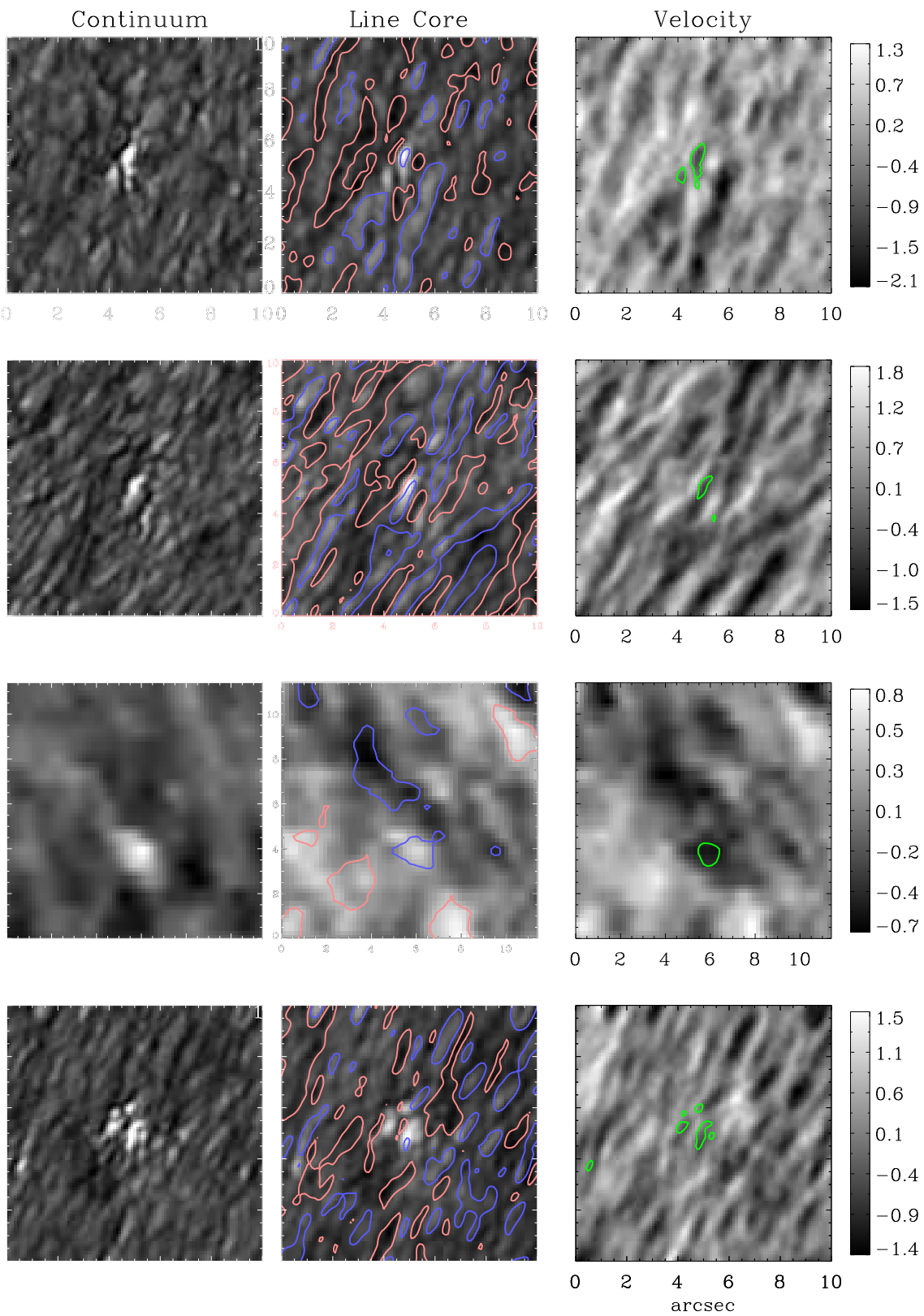

Figure 3.6: Intensity and line core velocity maps of areas with PFs, calculated from FPIVTT data and GCT data (second row from the bottom). The red contours in the line core images outline downflows (red shift) and the blue contours upflows. The contours in the velocity maps mark the locations of PFs. The units in the color bars are in $\mathrm{km} / \mathrm{s}$. Negative velocities are coded with dark color and correspond to velocities towards the observer. 

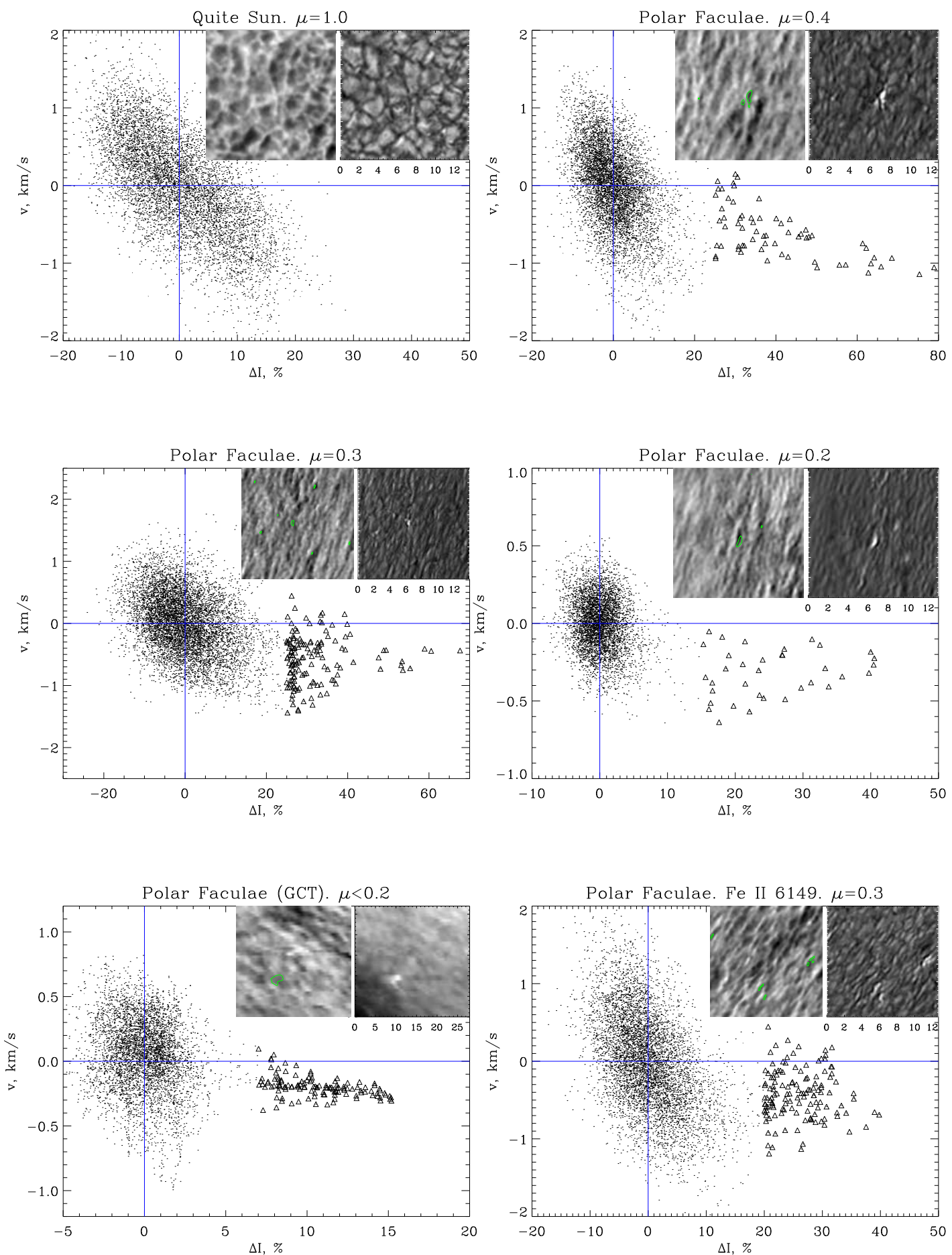

Figure 3.7: Relation between continuum intensities and line core LOS velocities in PFs and ambient photosphere. Four upper panels: FPI-VTT data in Fe I $6302.5 \AA$ line at different heliocentric angles. Lower panels: additional examples in GCT data and in other spectral line. Each panel contains velocity and continuum intensity maps from which the presented relations were calculated. Triangles correspond to PFs. 


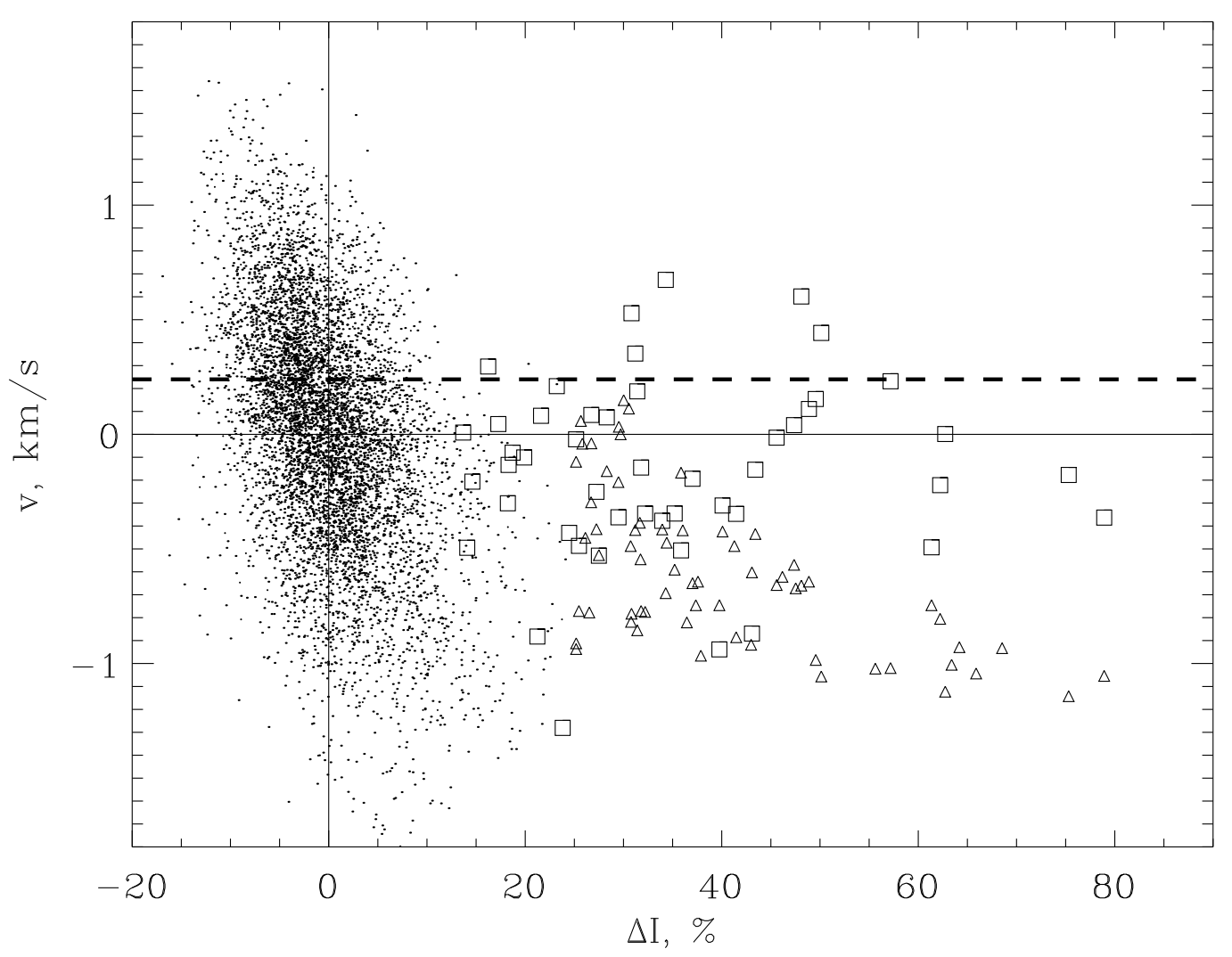

Figure 3.8: LOS velocities measured in Fe I $6302.5 \AA$ line at $\mu \approx 0.4 v$ s intensity contrast. The dots stem from the non-magnetic region within the $F O V$, the squares from the zerocrossings of the $V$ profiles of the facular points, and the triangles from their shifts of line center of $I$ profiles. Negative velocities are towards the observer. The horizontal dashed line at $+0.24 \mathrm{~km} \mathrm{~s}^{-1}$ gives the absolute zero velocity, rest at the solar surface, according to Schröter (1956).

Another possibility to obtain LOS velocities is from the zero-crossing of the Stokes $V$ profiles, which emerge from the magnetic plasma only, and therefore are free from nonmagnetic contamination. The difficulty here lies in the high requirements on the SNR and the spectral quality of the spectra. For zero-crossing analyses we have taken the best scan observed with the FPI spectrometer in the VTT at $\mu \approx 0.4$ in the $\lambda 6302.5$ line. The zero-crossings were measured from the Gaussians fitted to the $V$ profiles as described in Sect. 2.3. Following Schröter (1956, Eq. 37 there) we have corrected the reference zero velocity for $\mu \approx 0.4$, which contains, for medium strong lines, a residual, granular blueshift corresponding to approximately $-0.24 \mathrm{~km} \mathrm{~s}^{-1}$, relative to rest on the solar surface. Fig. 3.8 gives a comparison of zero-crossing (squares) and line core (triangles) velocities. The upflows in PFs are also found in zero-crossing velocities. Yet, the scatter of these velocities is large, $\pm 1 \mathrm{~km} \mathrm{~s}^{-1}$. This is not due to noise, i.e. random measurement errors, which were estimated to be less than $0.25 \mathrm{~km} \mathrm{~s}^{-1}$. It is inherent to the properties of individual flux tubes and reflects their dynamic behavior. 
The average velocity of the gas within the flux tubes is $-(0.15 \pm 0.06) \mathrm{km} \mathrm{s}^{-1}$. The velocity relative to rest on the solar surface then amounts to $-0.39 \mathrm{~km} \mathrm{~s}^{-1}$. This value is likely to be even more negative, towards the observer, since the zero-crossings tend to be biased towards redshifts on the following ground: Most $V$ profiles exhibit stronger blue than red lobes. The convolution with the FPI spectrometer's Airy function then yields, according to numerical tests, a redshift of the $V$ zero-crossings corresponding to 0.2 $0.5 \mathrm{~km} \mathrm{~s}^{-1}$.

The high velocities of $0.5-1.0 \mathrm{~km} \mathrm{~s}^{-1}$ towards the observer from the $I$ profiles (triangles in Fig. 3.8) are surprising. We note that such values are found in all our high spatial resolution observations. They are different from the velocities determined from the $V$ zero-crossings and thus refer mainly to bright gas surrounding the magnetic fields. This is difficult to comprehend in view of numerical MFT simulations where it is found that small-scale flux tubes are embedded in regions of downflowing cool gas (Deinzer et al. 1984a, 1984b; Vögler \& Schüssler 2003).

The velocities towards the observer within the magnetic structures are especially interesting because they are probably related to the fast solar wind above coronal holes. Let us estimate the required mass flow. We ascribe the measured velocities of $v_{\mathrm{FT}} \approx 1.0 \mathrm{~km} / \mathrm{s}$. The average proton density in the solar wind at 1 AU according to Denskat (1982) is 3 $4 \mathrm{~cm}^{-3}$. Assuming a wind speed of $800 \mathrm{~km} / \mathrm{s}$ (Stix $\left.2002 \mathrm{p} .406\right)$ and an area of $\pi \times(1 \mathrm{AU})^{2}$ we estimate the proton flow in the fast solar wind as

$$
\mathcal{N}_{\mathrm{p}}=n_{\mathrm{p}} v_{\mathrm{p}} A_{\mathrm{cor}} \approx 1.7 \times 10^{35} \mathrm{~s}^{-1}
$$

The hydrogen density at the formation height of Fe I $6302.5(\sim 350 \mathrm{~km}$ in the VAL C model, Vernazza et al. (1981), accounting for $\mu \approx 0.4$, is $n_{\mathrm{H}} \approx 1.0 \times 10^{16} \mathrm{~cm}^{-3}$. The flow $\mathcal{N}_{\mathrm{H}}$ of hydrogen particles in one PF with an area covered by magnetic fields of $A_{\mathrm{FT}} \approx(350 \mathrm{~km})^{2}$ is then

$$
\mathcal{N}_{\mathrm{H}}=n_{\mathrm{H}} v_{\mathrm{FT}} A_{\mathrm{FT}} \approx 1.2 \times 10^{36} \mathrm{~s}^{-1}
$$

Obviously, PFs can feed the fast solar wind above a polar coronal hole with a more than sufficient mass flow. Our finding needs further observational validation and comparison with equatorial faculae.

\subsubsection{Amplitudes of the polarimetric signal}

Polarimetric observations are the only way to study the magnetic structure of PFs, which are still unresolved by modern magnetographs and in spite of their kilo-Gauss fields do not produce splitting of spectral lines in the visible spectral range. As it was already mentioned, our observations contain only the $V$ component of the Stokes vector, the component which describes the circular polarization of the light and is thus sensitive only to the LOS projection of the magnetic field vector. The reduction of Stokes $V$ spectrograms requires high accuracy and is quite complex as described in detail in Chap. 2. In the present section we give a summary of the amplitudes of Stokes $V$ profiles emerging from PFs, whose knowledge are important for further numerical forward modeling of the facular structures. 
Table 3.1: Stokes $V$ amplitudes measured in polar faculae

\begin{tabular}{c|ccc}
\hline Spectral line & Telescope & $\mu=\cos \theta$ & $V / I_{\mathrm{C}, \mathrm{QS}}$ \\
\hline & & & \\
6302.5 & FPI-VTT & 0.4 & $\sim 0.06$ \\
6302.5 & $\ldots$ & 0.3 & $\sim 0.03$ \\
6302.5 & $\ldots$ & 0.2 & $\sim 0.025$ \\
& $\ldots$ & & \\
6149.3 & $\ldots$ & 0.3 & $\sim 0.01$ \\
6301.5 & $\ldots$ & 0.3 & $\sim 0.02$ \\
\hline & & & \\
6301.5 & GCT & 0.3 & $\sim 0.018$ \\
6302.5 & $\ldots$ & 0.3 & $\sim 0.024$ \\
6301.5 & $\ldots$ & 0.2 & $\sim 0.015$ \\
6302.5 & $\ldots$ & 0.2 & $\sim 0.019$ \\
\hline
\end{tabular}

Stokes $V$ profiles were normalized to the continuum intensity close to the spectral lines from the nearby, undisturbed photosphere $\left(V / I_{\mathrm{C}, \mathrm{QS}}\right)$, and some examples of $V$ profiles measured in PFs in all three iron lines are presented in the lower panels of Figs. 3.4 and 3.5. The amplitudes vary from facula to facula and depend on many factors, such as, for example, magnetic sensitivity of the spectral line, position of PF on the disk, resolution of the observation (filling factor). Typical amplitudes of $V$ profiles from PFs are collected in Table. 3.1.

The amplitudes of Stokes $V$ exhibit the expected CLV. They are decreasing towards the limb. This simply reflects the fact that magnetic fields in PFs are mainly vertical and when observations are approaching the limb magnetic flux tubes which constitute PFs are seen more and more obliquely to the line-of-sight. However at large heliocentric angles the ray along the LOS starts to cross several MFTs, which also contribute to the emerging magnetic signal, but because of their tiny size and tilted fields the Stokes $V$ amplitudes near the limb are small.

The strongest measured $V$ signal $(\sim 0.06)$ emerges from the very bright PF observed fairly far from the limb $(\mu=0.4)$ and in the most magnetically sensitive spectral line Fe I 6302.5 ( $g=2.5)$. Such a degree of circular polarization, relatively high for unresolved fields, is due to the large distance of the facula from the limb and probably due to a high filling factor peculiar for large structures. More frequent values of $V$ amplitudes measured in PFs close to the solar limb are in the range of 0.01 to 0.03 .

It is important to note that the Stokes $V$ amplitudes measured with both telescopes are substantially underestimated, because of the limited spatial and spectral resolution. Observations with the GCT are characterized by approximately $1^{\prime \prime}$ spatial resolution and 0'.19 pixel size, while reconstructed spectropolarimetric data from the FPI-VTT have two times better resolving power: $\sim 0$ " .5 spatial resolution and $0{ }^{\prime \prime} 1$ pixel size. Accordingly, the effective filling factor $f_{\text {eff }}$, defined as the ratio of the area occupied by magnetic structures to the area of the resolution element, is approximately $3-4$ times bigger for the reconstructed FPI-VTT data than for the GCT observations. However, we do not measure in the VTT data 4 times stronger Stokes $V$ amplitudes than in the GCT spectrograms. 
This is due to the peculiarities of the transmission of the FPI spectrograph (Sect. 2.2.1), which reduces the spectral line depression and accordingly the amplitudes of Stokes $V$ profiles. But even after this degradation the polarimetric signal recorded with the $2 \mathrm{D}$ spectrograph is still systematically stronger than in the GCT observations, which have high spectral quality but lower spatial resolution.

\subsubsection{Calibration of magnetograms}

We need to calibrate our polarimetric observations, i.e. to obtain an estimation of the true magnetic field strength in PFs from the derived properties (separations, amplitudes, shapes) of the observed Stokes profiles. For this purpose a number of diagnostics were developed during the recent decades, which allow to estimate approximately magnetic field strength or flux without involving complicated calculations. An exhaustive description of them is given by Solanki (1993). These calibration techniques are based on several simplifying assumptions, such as constant magnetic field and plane parallel atmosphere, and usually are applied for interpretation of observations at the center of the disk, where the above assumptions appear to be reasonable at least in the range of heights where the observed spectral line under analysis is formed and where the magnetic field can be considered as constant, or slowly varying. Near the limb the atmosphere along the LOS is very intermittent in the presence of MFTs, which makes the spectral line formation process complex and the interpretation of polarimetric observations not straightforward. Nevertheless, before proceeding to the next chapter, where the peculiarities of the observations near the limb will be treated accurately by means of numerical modeling, we will try to apply directly some of the known diagnostics to the observed data in order to get an idea about field strengths in PFs.

Magnetic field strengths can approximately be estimated from the amplitudes (weak field approximation) and the separations (strong field regime) of Stokes $V$ profiles, or combining both and using synthetic calibration curves. All three possibilities will be briefly sketched in this section.

As usually we introduce the Zeeman splitting $\Delta \lambda_{B}$ defined as

$$
\Delta \lambda_{B}=\frac{e}{4 \pi m c^{2}} g_{\mathrm{eff}} \lambda^{2} B
$$

where $g_{\text {eff }}$ is the Lande factor, a property of the transition characterizing the magnetic sensitivity of a spectral line; $\lambda$ is the central wavelength of the transition, and $B$ the magnetic field strength. The other coefficients have their usual meaning. In more convenient numerical form we can write

$$
\Delta \lambda_{B}=4.67 \times 10^{-13} g_{\mathrm{eff}} \lambda^{2} B,
$$

where $B$ should be given in $\mathrm{G}, \lambda$ and $\Delta \lambda_{B}$ in $\AA$.

Weak field apporximation: In the weak field approximation (WFA) the Zeeman splitting of the spectral line under consideration is smaller than the Doppler width of the line: $\Delta \lambda_{B} \ll \Delta \lambda_{D}$. In this case, the measured separation $\Delta \lambda_{V}$ of the Stokes $V$ lobes is approximately equal to $\Delta \lambda_{D}$ and is insensitive to the strength of the field. With increasing $B$ the separation $\Delta \lambda_{B}$ will not change, and the amplitude will grow linearly: $B \propto V_{a m p}$. 

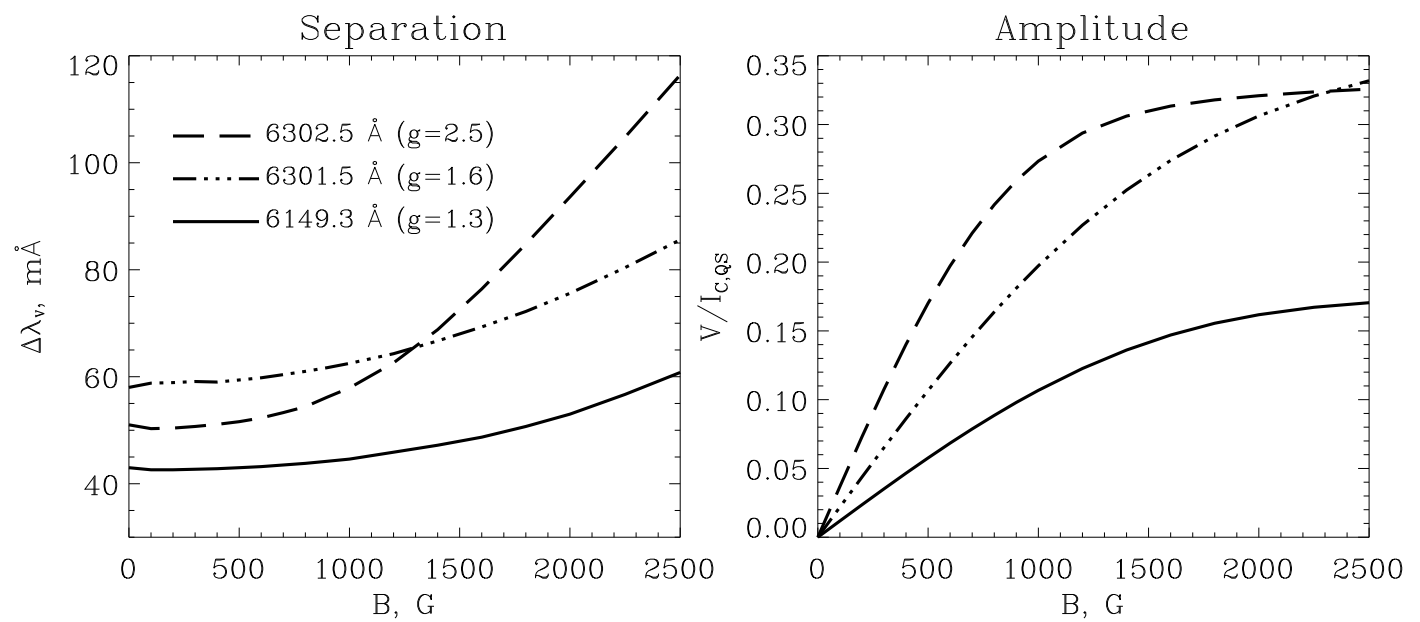

Figure 3.9: Separations and amplitudes of synthetic Stokes $V$ profiles for three iron lines. Note that there is an offset in the ordinate of the left panel.

Different atomic transitions are characterized by their own ranges of magnetic field strengths, where the WFA is applicable. To simulate the behavior of observed spectral lines in the presence of magnetic field a set of synthetic Stokes $V$ profiles for each transition was calculated. The radiative transfer problem was solved numerically under the assumption of a plane-parallel atmosphere, whose parameters are given by the VALC photospheric model (Vernaza et al. 1981), constant magnetic field parallel to the line-of-sight and with field strengths in the range from 100 to 2500 G. Separations and amplitudes were determined from synthetic profiles (Fig. 3.9). We can see that the lines Fe II 6149.3 and Fe I 6301.5 are most appropriate for the calibration of polarimetric data in the WFA, the first one due to its small Landé factor $(\mathrm{g}=1.3)$ and second due to the relatively large width of the specral line $(\sim 60 \mathrm{~m} \AA)$. Therefore the linearity of the $V$ amplitudes for these two lines is kept for a bigger range of $B$ than for the Fe I 6302.5 line, which is more magnetically sensitive.

Expanding Stokes $V$ in a Taylor series, expressing it in terms of intensity and neglecting in the expansion quadratic and higher order terms in $\Delta \lambda_{B}$ (Stenflo 1984) the formula for calibration in WFA can be obtained as

$$
V(\lambda) \sim \cos \gamma \Delta \lambda_{B} \frac{d I_{m}(\lambda)}{d \lambda}
$$

where $\gamma$ is the inclination of the magnetic field to the line-of-sight, and $I_{m}$ the unsplit intensity profile from the magnetic atmosphere. The observed Stokes $I$ is a good approximation to $I_{m}$ in case of weak fields. Rewriting Eq. (3.6) in terms of the amplitudes we get

$$
B \cos \gamma \sim C \frac{V_{a m p}}{\left(I^{\prime}\right)_{a m p}}
$$

where $C=\left(4.67 \times 10^{-13} g_{\text {eff }} \lambda^{2}\right)^{-1}$ is constant and $\left(I^{\prime}\right)_{a m p}$ is the amplitude of the derivative of Stokes $I$. 
Table 3.2: Average magnetic flux density in G in PFs estimated in WFA

\begin{tabular}{c|ccc} 
& & & \\
Spectral line & Telescope & $\mu=\cos \theta$ & $B_{a p p}$ \\
\hline 6149.3 & VTT & $\sim 0.3$ & $\sim 250-300$ \\
6301.5 & GCT & 0.2 & $\sim 100$ \\
6302.5 & GCT & 0.2 & $\sim 80$ \\
\hline
\end{tabular}

To apply this calibration technique to our low signal data and in case of FPI-VTT observations to spectra with moderate spectral sampling, we have proceeded in the following way. For each pixel in the field of view the derivative of the Stokes $I$ profile was evaluated numerically. Afterwards, to reduce the noise both the corresponding Stokes $V$ profile and derivative of Stokes $I$, which has the shape of $V$ profiles, were fitted to two Gaussian function and a polynomial by a non-linear least squares method as described in Sect. 2.3. The amplitudes of both fitted functions were determined as half of the absolute intenisty difference between two extrema and substituted in Eq. 3.7. Since the calibration is applied to unresolved fields the method returns not the real strengths of the magnetic fields but only the flux density or apparent field strength $B_{a p p}=f B \cos \gamma$, an average magnetic flux per square unit. Here, $f$ is the area filling factor which expresses the coverage of the observational element by magnetic fields. The estimated values were expressed in Gauss units, where $1 \mathrm{G}=1 \mathrm{Mx} / \mathrm{cm}^{2}$ and some examples are given in Table 3.2.

Flux densities derived from high spatial resolution data, observed with the FPI-VTT and corrected for atmospheric distortions, are systematically larger than from GCT data, which have lower resolution and accordingly smaller effective filling factors.

With the GCT both lines of neutral iron were recorded simultaneously with high spectral quality. This allows to compare the WFA with two lines. It was found that the flux density derived from the 6301.5 line is always bigger than that from 6302.5.

The average ratio was $B_{a p p}(6301.5) / B_{a p p}(6302.5)=1.28$.

However, if the WGA is correct both estimations should coincide. The same ratio and the same reasoning was presented by Domínguez Cerdeña et al. (2003) for the interpretation of polarimetric observations in quiet Sun disk center. So, we can also conclude that that the WFA is not valid for PFs, at least for one of the two spectral lines. Obviously it fails for the transition Fe I 6302.5, which is more magnetically sensitive than the other line.

Strong field regime: In the strong field regime the Zeeman splitting is bigger than Doppler width of the spectral line $\left(\Delta \lambda_{B}>\Delta \lambda_{D}\right)$, and the Stokes $V$ amplitude is no longer proportional to $B$. Stokes $V$ profiles are saturated and an increase of $B$ will change only the separation of the Stokes $V$ lobes but not the amplitude. We define separation $\Delta \lambda_{V}$ as the half distance between the extrema of the lobes of a $V$ profile. In the case of strong field $\Delta \lambda_{V}$ is close to the Zeeman splitting $\Delta \lambda_{V} \sim \Delta \lambda_{B}$ (Eq. 3.5).

An estimation of the field strength from the separation of Stokes $V$ extrema has the advantage that it is almost insensitive to the amplitude of the $V$ profiles, which are usually strongly reduced by an a priori unknown filling factor and in case of data obtained with the FPI-VTT also substantially reduced by the Airy function of the spectrometer. The decrease of the Stokes $V$ extrema on the above grounds should not strongly affect the location of the peaks. However this technique should be used with care, it is applicable only for magnetically very sensitive lines in strong field. 
For calibration in the strong field regime we have used polarimetric data observed in the Fe I 6302.5 line as the most appropriate for the assumption of the strong field regime. It has a large Landé factor $(g=2.5)$ and relatively small width of the line profile $(\sim 50 \mathrm{~m} \AA)$, so starting already from 1 kilo-Gauss field strength it is close to the strong field regime and at $B=1.6$ kilo-Gauss the Stokes $V$ profiles are completely saturated.

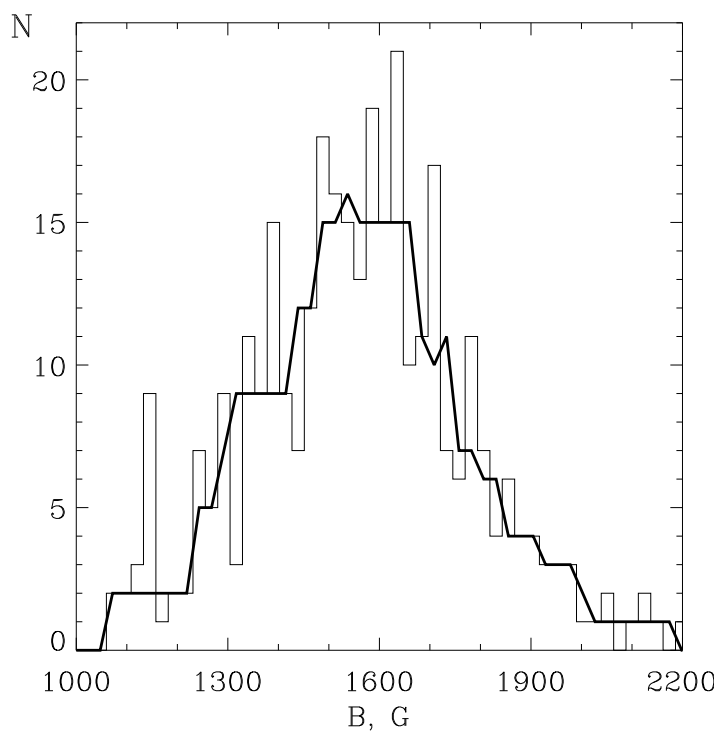

Figure 3.10: Histogram of magnetic field strengths derived from separation of Stokes $V$ lobes in Fe I 6302 line.

Replacing $\Delta \lambda_{B}$ in Eq. (3.5) by measured separation $\Delta \lambda_{V}$ we get for the $\lambda 6302.5$ line numerically $B=\Delta \lambda_{V} / 46.2$, where $\Delta \lambda_{V}$ should be given in $\mathrm{m} \AA$ and $B$ will be in kiloGauss. We have applied such a calibration to the FPI-VTT observations. Stokes $V$ profiles were fitted with an analytical function by a non-linear least squares method (Sect. 2.3). In Fig. 3.10 we present the result of this calibration. Only measurements from pixels belonging to PFs entered the histogram. The maximum of the distribution of magnetic field strengths is around $1600 \mathrm{G}$. This estimation is in good agreement with the previous measurements of Homann at al. (1997).

We note the existence of strongly separated Stokes $V$ profiles from polar faculae with $\Delta \lambda_{V} \geq 90 \mathrm{m \AA}$, resulting in unusually high magnetic field strengths of about $2000 \mathrm{G}$ (Fig. 3.10). It was recently shown by Bellot Rubio (2004) that such a calibration based on the estimation of the separation of Stokes $V$ extrema can lead to a systematic overestimation of the field strength, if the magnetic field lines are strongly inclined to the LOS. The suggested explanation infers magnetooptical effects, i.e. the transformation of a part of linearly polarized light into circularly polarized radiation. Although this verification was made for a penumbral model, we can expect a similar effect in facular atmospheres, because near the limb vertical flux tubes are seen tilted and the effect of the linear polarization should be taken into account.

Synthetic calibration curves. In this section we present another possibility to calibrate polarimetric observations without involving complicated calculations. The method requires both separations and amplitudes of the observed $V$ profiles and is based on the comparison of the combination of both characteristics of polarimetric signal with the values derived from a set of precalculated synthetic Stokes $V$ profiles.

To apply this calibration we have chosen high quality spectrograms containing a very bright and large PF observed with the FPI-VTT in the $\lambda 6302.5$ line. The facula was located at $\mu \sim 0.4$ and produced the strongest $V$ signal from the whole observational set obtained in the framework of this study. To improve the statistical sample the best 9 scans from the recorded time sequence were selected. In order to separate magnetic fields in the PF from other photospheric fields we have applied for each scan a mask, containing 

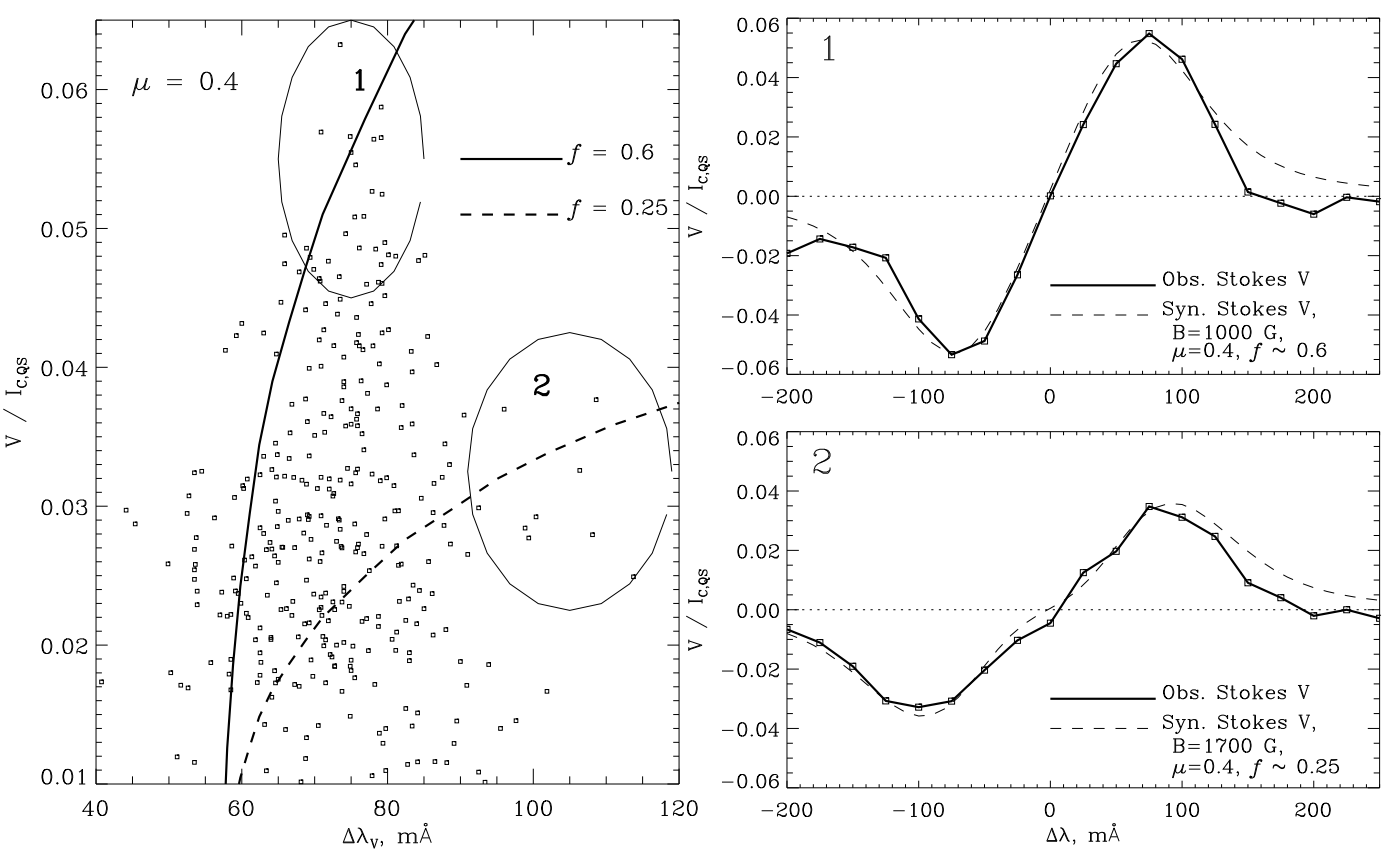

Figure 3.11: Left panel: Amplitudes vs separations of peaks of Stokes $V$ profiles observed (open squares) in PF at $\mu=0.4$ in Fe I 6302.5 overplotted with calibration curves (dashed and solid lines) calculated from simple theoretical model with the assumption of plane parallel photosphere and constant magnetic field. See the text for more details. Right panels: Comparison of observed Stokes $V$ profiles averaged in groups 1 and 2 (from the left panel) with the synthetic ones.

only pixels with the brightness above $3 \sigma$ of the surrounding photospheric intensity fluctuations. Then, as usually, individual $V$ profiles were fitted with an analytical function to improve the precision of the definition of separations and amplitudes. The latter were estimated and plotted as a scatter diagram $\Delta \lambda_{V} v s V$ (Fig. 3.11 left panel, open squares). The separations vary from $50 \mathrm{~m} \AA$ to $110 \mathrm{~m} \AA$ and amplitudes from $1 \%$ to $6 \%$ in units of the continuum intensity of the quiet Sun. It was found that the measurements with the strongest $V$ amplitudes (Fig. 3.11 left panel, group 1) do not coincide with most separated profiles (group 2), as one would have expected from the simple theoretical model with constant filling factor.

For diagnostics synthetic Stokes $V$ profiles were calculated from a simple model with the following assumptions: VALC (Vernazza et al. 1981) plane parallel photosphere, constant and homogeneous magnetic field, inclination of magnetic field corresponds to the heliocentric angle of observed PF $\mu=\cos \theta=0.4$. To simulate the instrumental degradation of $V$ profiles due to the limited spectral resolution of the FPI the synthetic profiles were convolved with the transmission curve of the spectrograph given by the analytical expression of the Airy function (Koschinsky 2001). This way the $V$ profiles are reduced by a factor of typically 1.5 . It is interesting to note that the convolution with the Airy function also slightly changes the separation of the $V$ extrema. They are systematically increased by few percent. This is due to the fact that the lobes of $V$ profiles are not symmetric, they have extended wings away from line center. Finally, to consider 
limited spatial resolution of the telescope the amplitudes of synthetic Stokes $V$ profiles were multiplied by filling factors 0.6 and 0.25 (Fig. 3.11, left panel solid and dashes lines). These values were chosen in order to cover with the theoretical curves the observations with both the most separated $V$ lobes and strongest amplitudes.

The measured combinations of $\Delta \lambda_{V}$ and $V_{a m p}$ do not fit completely to any of the calibration curves, although most of the points are found between the two plotted curves. Obviously, the effective area filling factor is varying across the area occupied by the polar facula, which was observed with high spatial resolution.

Profiles belonging to groups 1 and 2 in Fig. 3.11, left panel, were averaged to reduce the noise. The best fits of the theoretical Stokes $V$ profiles were found simply by varying the constant magnetic field (to fit the separation) and the filling factor (to fit the amplitude). The results are plotted in the right panel of Figure 3.11. Especially remarkable is the profile averaged in group 2. It is definitely in the strong field regime with $\Delta \lambda_{V}=100 \mathrm{m \AA}$, the corresponding synthetic $V$ profile $(B=1.7$ kilo-Gauss $)$ reveals even magnetooptical effects, which however disappear when we convolve the profiles with the transmission of the spectrometer.

Another important result from such calibration which combines both separations and amplitudes of observed polarimetric signals is that it allows to estimate the filling factor, which for this particular PF is in the range of 0.2 to 0.6 . Especially reliable appears the estimation of the filling factor in the case of strong fields when $V$ amplitudes saturate and where the measured amplitude depends only on the spectral line strength, filling factor and not on the magnetic field strength.

\subsection{Spatial distribution of the magnetic field in PFs}

High spatial resolution of the observations with the Fabry Perot spectrometer in the VTT ( 0.5 in narrowband and 0.25 in broadband), achieved by means of image restoration, allows to study in detail the spatial distribution of the polarimetric signal in PFs. We have analyzed the positional relationship between continuum brightness of polar faculae in speckle reconstructed broadband images and the amplitudes of the Stokes $V$ signal.

It was found that in general magnetic field and intensity in PFs are co-spatial, i.e. polarimetric signals emerge from the PF brightening. As it is shown in Fig. 3.12 the contours of the lowest detectable Stokes $V$ signal (blue dotted lines) almost totally encircle the PF brightenings. It is especially well seen in the left panel of Fig. 3.12 corresponding to observations in the $\lambda 6302.5$. The $\lambda 6149.3$ line (right panel) generates weaker $V$ signal, though the detection limit (noise level) for both observations is the same. As a result, the lowest detectable polarimetric signal from the $\lambda 6149.3$ line covers only a part of the PF.

Remarkably, the strongest polarimetric signal is systematically displaced in the direction to the disk center with respect to the continuum intensity by a fraction of arcsec (red solid contours in Fig. 3.12). This is an interesting result which will be used for verification of the reliability of numerical models in forthcoming sections, devoted to simulations of faculae structures at large heliocentric angles. 

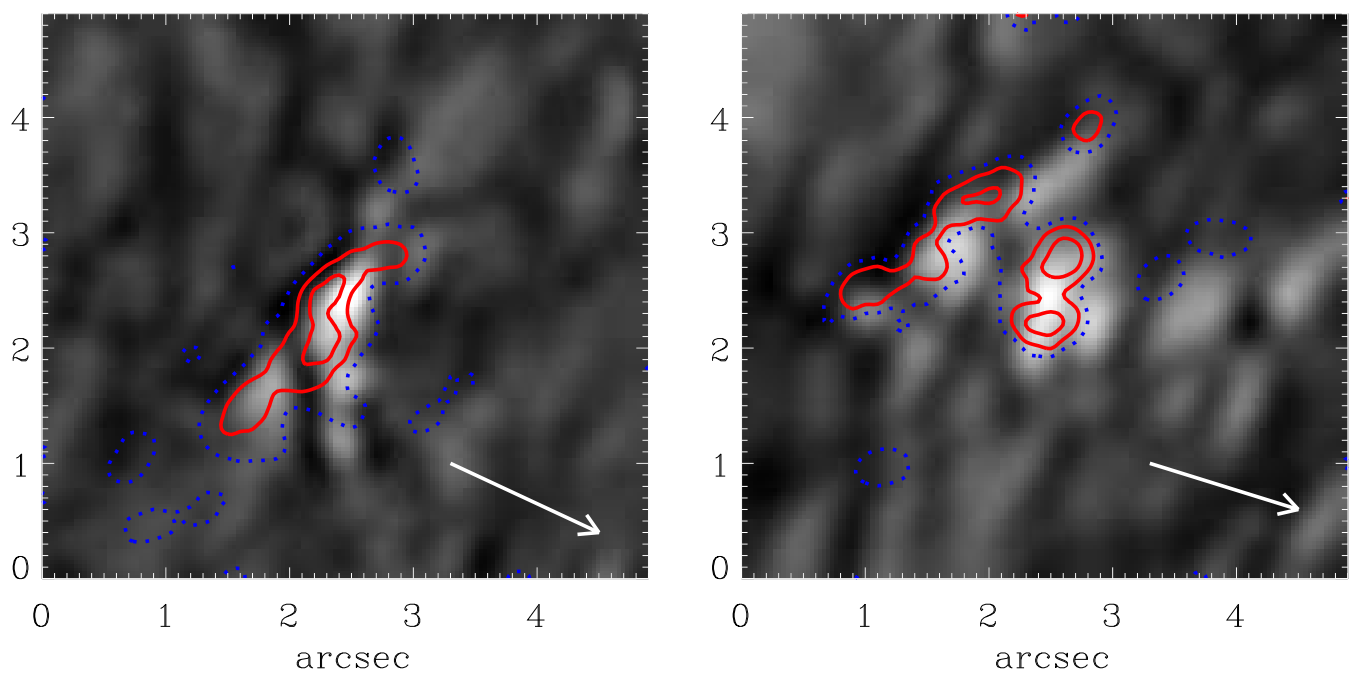

Figure 3.12: Continuum intensity maps overlaid with contours locating the origin of the strongest polarimetric signal (red solid contours) and the lowest detectable $V$ signal (blue dotted contours) measured in PFs in the $\lambda 6302.5$ (left image) and in the $\lambda 6149.3$ line (right image). Arrows point to the limb.

\subsection{Magnetic polarity of polar faculae}

Analysis of the magnetic polarity of polar faculae is important for understanding the relation of PFs to the global magnetic field of the Sun and their place in the hierarchy of solar magnetic fields. Plage faculae seen near the limb at low latitudes belong to usually bi-polar active regions, which are a manifestation of the toroidal component of the global field. Network fields also do not show any preferable polarity. Polar faculae are supposed to have another origin, and as a part of the poloidal component of the global magnetic field, they are expected to have corresponding to the hemisphere polarity.

According to Homann et al. (1997) PFs are unipolar and their magnetic polarity is that of the general polar magnetic field. However, Zhang \& Zhang (1999) have reported on no preference of polarity in small-scale magnetic structures at the solar poles, the authors found that magnetic structures are often organized in bi-polar pairs. In this section we present our measurements of the magnetic polarity in PFs.

Polarity of solar magnetic field during observations. The observational material for this study was taken during several campaigns in the years 2000-2002, close to the maximum of the current magnetic activity cycle (cycle 23). The cycle started in 1997 and reached its maximum at the end of 2000, when the reversal of the poloidal magnetic field occurred. The bi-polar groups in active regions follow Hale's polarity law, i.e. during the present cycle in the northern hemisphere the leading sunspots have positive polarity and the following ones have negative polarity, and vice versa for the southern hemisphere (Fig. 3.13).

Following the convention we will call positive (or north) magnetic polarity the one with magnetic field directed to the observer. Negative (south) polarity is accordingly directed to the Sun. Usually north polarity is coded on magnetograms with white color and south in black color. 
Years of the maximum solar activity correspond to the epoch of the polar field reversal. Before the reversal, during the growth of sunspot activity, the polarity of the North Pole was northern (positive), after the reversal which finished by the end 2000, beginning of 2001, it became southern, as it is schematically shown in Fig. 3.13. The reversal is not an instant process, it can take several months, during which mixed polarities can be found at solar poles (Fig. 1.1).
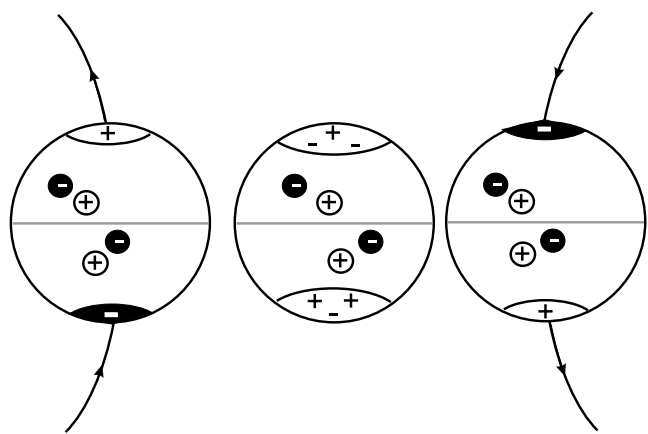

Figure 3.13: Polarities of the solar large scale magnetic field before, during and after (from left to the right) the reversal of the polar field in current 23 activity cycle.
Observations and calibration. All observations were made in the years close to the maximum of solar activity in sunspots and accordingly during minimum of polar activity, resulting in an unfortunately rare appearance of PFs at high latitudes. Therefore, to study polarities of PFs we have used all observations obtained with both telescopes (VTT and GCT), including data which were obtained under relatively poor seeing conditions, thus not considered for analysis before. The determination of the polarity does not require perfect imaging, and often can be derived from data with moderate quality.

The observed PF samples can be divided into two groups: data taken

1. during the reversal in December 2000;

2. after the reversal of the polar magnetic field in summer 2001 and spring 2002.

Spectrograms from the first group were recorded with the GCT and contain two observations near the north pole (NP) and one observation near the south pole (SP). In group two we have observed with the GCT two PFs at NP and two at SP, and with the VTT two at $\mathrm{NP}$ and 5 at SP. Comparing $V$ profiles recorded with the same setup with each telescope one can find if PFs appeared to be unipolar or not. But to compare observations from two telescopes with each other and to find the relation to the global magnetic field we have performed an absolute calibration of the measured polarities.

During the observational campaigns several sunspots were observed using the same configuration of the observational setup. Afterwards these sunspots were identified using intensity images and magnetograms of the MDI instrument on board of SOHO, and the relation of the observed magnetic signature to the commonly used definition of polarity was obtained. This calibration was performed for both telescopes.

Results. From the measurements of the polarities of PFs in group two, observed after reversal, it was found without any exception, though with a limited statistical sample (11 PFs were analyzed), that PFs are unipolar, i.e. all detected magnetic signals associated with bright facular points have the same polarity in each hemisphere. And this polarity is the one of the global poloidal magnetic field of the Sun, in our case it was north polarity in the northern hemisphere.

We explain the discrepancy between our results and those of Zhang \& Zhang (1999) who found many bi-polar pairs at high latitudes in the following way. For the determination of the polarity of PFs it is important to use simultaneously taken white-light images and magnetograms in order to identify PFs. The intrinsic property of PFs is a very high 

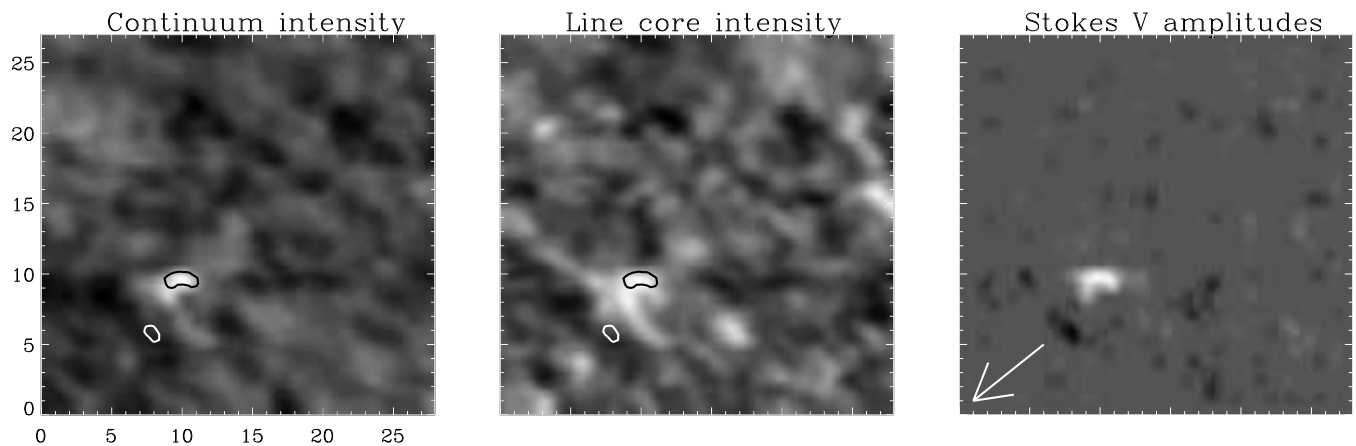

Figure 3.14: Intensity maps and magnetogram containing PF and surrounding photosphere. Left and middle images: continuum and line core intensity maps with contours outlining the location of strongest $V$ signal of both polarities. Right image: Stokes $V$ amplitude map with the arrow pointing to the limb and black and white colors denoting opposite polarities.

brightness contrast in high resolution images, which allow to distinguish PFs from the surrounding photosphere. Similar to the observations of Zhang \& Zhang, we have found that some of the analyzed PFs appear to have a patch of opposite magnetic polarity in the close vicinity (Fig. 3.14).

However, these patches show a behavior which is very different from PFs:

- they have very low intensity contrast;

- they exhibit weaker polarimetric signal;

- such bi-polar pairs are oriented in the center-limb direction with a opposite polarity patch closer to the limb, as it is shown in Fig. 3.14.

All these peculiarities allow to suggest that the patches of opposite polarity do not reflect the real magnetic polarity of PFs, but they are due to the horizontal component of the magnetic field in polar faculae, which at large heliocentric angles can cause the apparent reversal of the magnetic field (see e.g. Zirin 1999).

The use of continuum images is also important for distinguishing PFs from, for example, network faculae, which have smaller filling factors and as a result low contrasts, but can exhibit both polarities. The magnetogram in the right panel of Fig. 3.14 reveals that apart of PFs there exists a multitude of magnetic patches of both polarities but with much weaker Stokes $V$ amplitudes and low intensity contrasts.

The analyses of the data which were obtained during the epoch of the reversal in December 2000 (PFs from group 2) show that bright faculae points of both magnetic polarities and strong polarimetric signal can occur at polar caps of the Sun (Fig. 3.15). The observed picture agrees with the widely accepted scenario of the polar field reversal, which is based on a solar surface flux transport model (Wang \& Sheeley 2003, Dikpati et al. 2004). According to this scenario the trailing polarity from bi-polar active regions drifts towards the poles, where it cancels the opposite polarity from the previous cycle. 

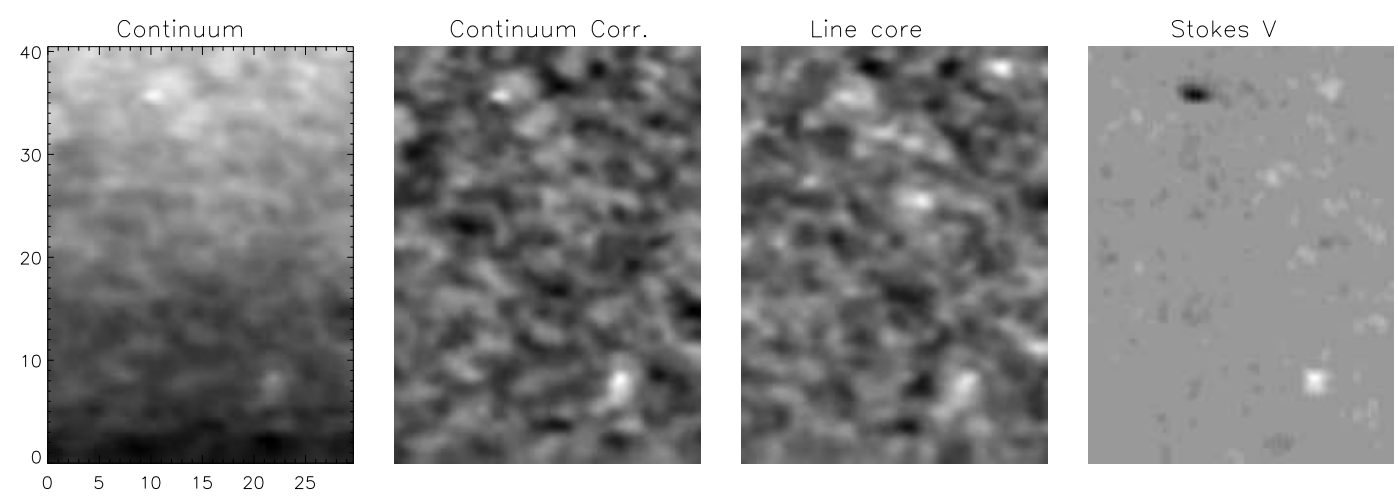

Figure 3.15: Isolated polar faculae of both polarities observed with GCT on 03.12.2000 during the epoch of the polar magnetic field reversal. Second from the left is the continuum image corrected for limb darkening.

To finish this section we briefly summarize the main results from the analyses of the magnetic polarity of polar faculae:

- polar faculae are unipolar with the magnetic polarity of the poloidial field;

- observed bi-polar pairs can be due to the apparent reversal of the inclined magnetic fields observed close to the limb;

- observed mixed polarities with weak polarimetric signal emerge from the surrounding photosphere (network magnetic fields);

- polar faculae of both polarities in the same hemisphere are observed during the epoch of the magnetic field reversal.

\subsection{Summary of the observational properties of PFs}

The present study deals with the observational properties of polar faculae on the Sun, which constitute an important part of the magnetic solar cycle. To reveal their rôle in the magnetic activity at the poles of the Sun we have concentrated in the above sections on the fine structure and the dynamics of PFs. Photometric and spectropolarimetric properties of polar faculae were gathered in order to create a "typical portrait" of facula structures observed near the polar caps of the Sun. For this purpose, we aimed at obtaining measurements in Stokes $I$ and $V$ of high spatial and spectroscopic resolution with the Gregory-Coudé Telescope and the Vacuum Tower Telescope at the Observatorio del Teide. The careful application of special methods of observation and data analysis, image speckle reconstruction and singular value decomposition, lead to the detection of a wealth of substructure and dynamic phenomena of PFs.

Before proceeding to the next chapters, completely devoted to theoretical studies of facular points by means of numerical modeling and radiative transfer calculations we summarize briefly the observational properties of the phenomena, which modeling should be able to reproduce. 
We summarize our main results, partly published in Okunev \& Kneer (2004), as follows:

- A polarimetric sensitivity of $|V| \approx 2 \times 10^{-3} I_{\mathrm{c}}$ at a spatial resolution of 0 '.5 was achieved. This revealed many small-scale, weak magnetic flux areas of both magnetic polarities surrounding the PFs proper, like in the quiet Sun near disk center (e.g. Domínguez Cerdeña et al. (2003)).

- PFs exhibit a high contrast of $\approx 50 \%$ at $\mu \approx 0.4$, which monotonically decreases still further to the limb, i.e. a maximum of the contrast at $\mu<0.4$ is not seen.

- PFs possess fine structure in both brightness and magnetic fields down to the spatial resolution limit ( $0^{\prime \prime} .25$ for speckle reconstructed broadband images and 0 '.5 for magnetograms). The broadband images from facular points appear somewhat displaced, $\sim 0$.'5, towards the limb with respect to the magnetic features.

- The life time of PF exceeds the duration of the observations of $\approx 1 \mathrm{~h}$. However, at small scales polar facular points are highly dynamic, they change noticeably their brightness and position within $50 \mathrm{~s}$.

- The intensities of Fe I and Fe II lines under study are higher in polar facular points than in the ambient atmosphere, throughout the line profiles. Yet relative to the local continuum, in the Fe II 6149.3 line the Stokes $I$ profiles from PFs $\left(I_{\mathrm{PF}}\right)$ are substantially stronger and broader than the averaged intensity profile from the surrounding quiet Sun photosphere $I_{\mathrm{QS}}$; in the Fe I 6301.5 line $I_{\mathrm{PF}}$ show almost the same line depression as $I_{\mathrm{QS}}$ but appear to be slightly broader than $I_{\mathrm{QS}}$; in the Fe I 6302.5 line $I_{\mathrm{PF}}$ is slightly weaker and broader than $I_{\mathrm{QS}}$.

- Calibrations of magnetic field measurements assuming weak field approximation and strong field regime were applied to observed polarimetric data, appropriate to the spectral lines. Both approaches reveal that the intrinsic magnetic field strengths of PFs are in the kilo-Gauss range, confirming thus the findings of Ritter (2001).

- Strong Stokes $V$ signals with amplitude up to $|V|=0.06 \times I_{\mathrm{c}}$ and separation of $V$ extrema $\Delta \lambda_{V} \approx 80 \mathrm{m \AA}$ are found in $\mathrm{Fe}$ I 6302.5 which require high area filling factors up to $f \approx 0.6$ and strong, kilo-Gauss fields.

- PFs are unipolar magnetic features, they possess the same magnetic polarity as the global magnetic filed. We thus confirm the result by Homann et al. (1997).

- From the shifts of line centers of Stokes $I$ profiles and from the zero crossing of Stokes $V$ we find an average upward flow of the plasma within the magnetic flux tubes of PFs of $v=0.5-1 \mathrm{~km} \mathrm{~s}^{-1}$, relative to rest on the solar surface, albeit with a large scatter reflecting the intrinsic, highly dynamic PF properties. This flow can feed the fast solar wind from polar coronal holes with sufficient mass.

We note that these results on PFs need further observational confirmation and should be contrasted with data from equatorial faculae when observed also near the limb. For the successful study of small-scale magnetic structures close to the limb observational facilities which combine high spectral and spatial resolution with a high spectropolarimetric sensitivity and low instrumental polarization are required.

The above listed properties are intrinsic to polar faculae, and therefore some of them can be used as observational constraints for the modeling of PFs. By comparison of the characteristic of simulated facula structures with the ones of observed faculae the adequacy of a numerical model can be tested. 


\section{Numerical modeling}

Modern observational facilities combined with sophisticated mathematical methods of data reduction provide a wealth of information about the structure and dynamics of the solar atmosphere. By analyzing data with high spectral and spatial resolution we have studied small-scale magnetic structures at high latitudes of the Sun. Their photometric and spectropolarimetric properties such as intensity distribution (CLV, brightness contrast), sizes and fine structure, dynamics, characteristics of the intensity profiles and polarimetric signals in three iron lines were established (Chap. 3). We have obtained from observations $I\left(x^{\prime}, y^{\prime}, \lambda, t\right)$ and $V\left(x^{\prime}, y^{\prime}, \lambda, t\right)$, i.e. two components of the Stokes vector as functions of wavelength, time, and spatial position in the plane perpendicular to the line-of-sight (LOS). But we need to retrieve physical parameters, such as temperature, gas pressure, magnetic field, velocities as functions of spatial coordinates in the solar atmosphere $(x, y, z)$. Applying standard calibrations one can only approximately estimate average values of these parameters (Sect. 3.3.4). Most methods of data interpretation are based on several simplifications (such as plane parallel atmosphere) which are not necessarily fulfilled in the solar photosphere. This holds especially for observations close to the limb, where the photosphere is very inhomogeneous along the LOS. It can lead to misestimating of the sought parameters. The importance of the detailed geometry of rays crossing very intermittent magnetic fields was shown by Solanki et al. (1998).

The result of the interpretation depends also on the adopted assumptions. For example the calibration of magnetograms depends strongly on the chosen approximation: weak field or strong field. Generally, we do not know a priori which one is appropriate. Furthermore, the area filling factor and the inclination of the magnetic field are two more unknown factors, which make the interpretation of the polarimetric observations even more difficult.

One solution is to apply numerical methods involving radiative transfer calculations, such as, for example, inversions of Stokes profiles (e.g. Ruiz Cobo \& del Toro Iniesta 1992). The method starts with the guess of the underlying atmospheric model and calculations of the corresponding intensity profiles and then some parameters of the initial model are modified until a best fit of the synthetic profiles to the observed ones is reached. Thus, by solving iteratively the inverse problem one obtains parameters of the atmosphere which may result in the observed intensity profiles. It is a powerful tool, which has been rapidly developed during the last decade and successfully applied to a wide range of problems in physics of the solar photosphere (e.g. Bellot Rubio et al. 2000, Borrero \& Bellot Rubio 2002, Puschmann et al. 2004). Yet, this technique poses high requirements on the quality of the data (high spectral resolution and high SNR). The inversion technique is a pure 1D approach, each single ray is considered independently and under the assumption of plane-parallel atmospheres a 1D radiative transfer problem is solved. 
Furthermore, to avoid time consuming calculations and for the sake of the convergence of the iterations only a limited small number of nodes (reference grid points along the integration path) are used for adjustment of the guess model. This peculiarity of the method makes it difficult to apply to problems where the atmosphere along the line-ofsight is very irregular and high coverage with grid points is mandatory. As it was already mentioned, the formation of a magnetically sensitive line close to the solar limb is characterized by a very intermittent atmosphere along the LOS, which requires many grid points along the integration path. Moreover, the observations obtained in the frame of the current study do not fully meet the requirements of the inversion technique, which can result in uncertainties of the calculated parameters.

For this study, we have chosen another approach known as forward modeling. The idea of the method is to create a physical semi-empirical model, controlled by several free parameters, to calculate emergent Stokes profiles $\boldsymbol{I}_{s y n}\left(x^{\prime}, y^{\prime}, \lambda\right)$ for several sets of the free parameters and to compare them with the observed profiles. The advantage of the forward modeling is that one can create a realistic two- or even three-dimensional model and test its viability. Moreover, the calculations can be performed with any required spatial resolution, both in directions along the LOS and perpendicular to it. And to simulate the limited spectral and spatial resolution of the observations, the synthetic profiles can be convolved with corresponding smearing functions.

Numerical modeling and radiative transfer calculations of the magnetic structures at large heliocentric angles with the LOS oblique to the axis of magnetic flux tubes have several peculiarities, which were discussed by Audic (1991), Bünte et al. (1993) and Solanki et al. (1998), who have performed such modeling. Radiative transfer calculations across flux tubes were also performed by Karpinsky \& Okunev (1998) to explain the observed fine structure of the brightness at the extreme limb of the Sun (Karpinsky \& Okunev 1997). These calculations were limited to the continuum intensity. In this work we extend the modeling to the radiative transfer of the full Stokes vector in order to interpret the high resolution spectropolarimetric observations of the facular structures at the solar poles. The aim of the study was not to find parameters of the atmosphere which give a best fit of individual line profiles, but to verify the hypothesis about PFs as concentrations of small-scale MFTs and to find the most adequate set of free parameters which will be able to reproduce the typical portrait of PFs, given by average observed properties of PFs.

In this chapter we describe the principles of direct modeling performed in the present work. Components and geometry of the model, calculation of atmospheric parameters, construction of the rays, numerical radiative transfer problems and spectral line synthesis in a magnetized plasma, as well as peculiarities of the numerical integration in very intermittent atmospheres will be discussed in the following sections. 


\subsection{Construction of the model}

The first stage of the numerical modeling, namely the construction of the simulation box ready for radiative transfer calculations, is described in this section.

\subsubsection{Basic assumptions: two-component static model}

The magnetic inhomogeneity of the facular atmosphere is modeled by embedding static magnetic flux tubes (MFT) in a field free medium. Such an assumption is supported by the observed properties of polar faculae. PFs were found to have fine structure both in magnetic field and intensity contrast and are characterized by kilo-Gauss fields and weak average flux density (Sect. 3.3.4). The latter is due to limited spatial resolution of the observations (small filling factors). Accordingly, the magnetic elements possess only very small diameters.

Thus, the model consists of two components: magnetic and non-magnetic, or internal and external with respect to the MFT. The components differ not only in the presence/absence of magnetic field but also in physical conditions, such as temperature, gas pressure, electron pressure, opacity, etc. For the external non-magnetic atmosphere we adopt the VALC model (Vernazza et al. 1981) extended to deeper subphotospheric layers using the model of the convective zone of Spruit (1977). The model covers a range of heights in the solar atmosphere from $z=-200 \mathrm{~km}$ to $600 \mathrm{~km}$, where $z=0$ refers, as usually, to continuum optical depth $\tau_{c}=1$ at $\lambda=5000 \AA$. The parameters of the internal atmosphere are given by the prescribed thermal stratification (parameter of the model) and the gas pressure obtained from the pressure balance between the magnetic and non-magnetic atmospheres (cf. Eq. 4.1 below). The magnetic structure of the internal atmosphere is defined by the adopted model of the magnetic flux tube (MFT).

Although it was found that polar faculae are highly dynamical structures of the solar photosphere, and that their fine structure evolves on very short time scales (Sect. 3.2) and the bright elements themselves exhibit pronounced upflows (Sect. 3.3.2), we restrict our modeling to the static case for the sake of simplicity. We adopt stationary MFTs in pressure balance with the external atmosphere.

In principle, we have the possibility to calculate from the created model LOS velocities for different heliocentric angles, but for this one would have to define all three components of the velocity vector for both components as functions of the three spatial coordinates: $\boldsymbol{v}_{\text {int }}(x, y, z)$ and $\boldsymbol{v}_{\text {ext }}(x, y, z)$. The selfconsistent assignment of the full velocity vector is not straightforward in $3 \mathrm{D}$ geometry, therefore in the presented modeling all macroscopic velocities were set to zero. Modeling of the shifts and asymmetries of Stokes $I$ and $V$ profiles are deferred to forthcoming studies, and the present work deals with photometric contrasts, line strengths and amplitudes of polarimetric signals.

\subsubsection{Model of a single magnetic flux tube}

The concept of small-scale MFTs as bricks which constitute unresolved magnetic fields in the solar atmosphere is a cornerstone of the presented numerical model. The adopted model assumptions are the usual for such calculations: axisymmetric vertical flux tubes 
are in pressure balance with the surrounding photosphere; the parameters of the internal atmosphere $\left(T, P_{g}\right)$ depend only on height and not on radius (thin tube approximation).

The external gas pressure is balanced by the internal gas and magnetic pressures, which yields within the thin flux tube approximation

$$
P_{g}^{e x t}(z)=P_{g}^{i n t}(z)+\frac{B^{2}(z)}{8 \pi}
$$

The external gas pressure in the solar photosphere is exponentially decreasing upwards, therefore, the magnetic field strength should follow the similar law to satisfy the condition of equilibrium (Eq. 4.1). Magnetic fields of flux tubes are predominantly vertical in the lower photosphere, and for the $z$ component of the field we assume:

$$
B_{z}(z)=B_{0} e^{-z / 2 H_{p}}
$$

where $B_{0}$, the magnetic field strength at $z=0$ is a free parameter of the model, and $H_{p}$ is the pressure scale height.

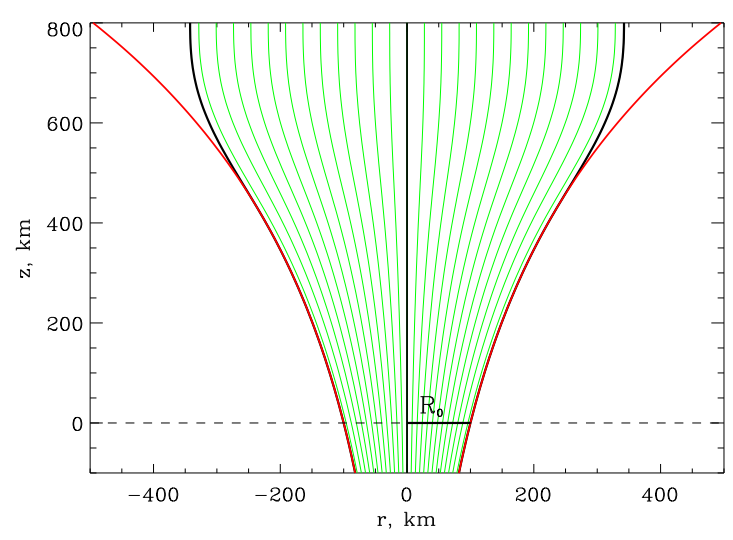

Figure 4.1: Axial section of the MFT in the ZX plane illustrating the configuration of the magnetic field of the flux tubes. Model parameter $R_{0}=100 \mathrm{~km}$. The expansion of the flux tube (red lines) is limited by the introduced largest attainable radius $R_{\max }=300 \mathrm{~km}$.

The second parameter of the model is the radius $\left(R_{0}\right)$ of the MFT at $z=0$. From the magnetic law of flux conservation $R(z)^{2} B(z)=R_{0}^{2} B_{0}$ together with Eq. 4.2 we can find the expansion of the flux tube:

$$
R(z)=R_{0} \sqrt{\frac{B_{0}}{B(z)}}
$$

Flux tubes are not strictly cylindrical, they are expanding upwards. Therefore, it will be more adequate to describe the magnetic field vector in cylindrical coordinates with two components, the vertical $B_{z}$ and the radial $B_{r}$. The azimuthal component $B_{\phi}$ is set to zero. To obtain $B_{r}$ we proceed in a way similar to Audic (1991). We assume that at the axis of a tube the magnetic field is strictly vertical and the radial component vanishes $\left(|B(r=0)|=B_{z}\right)$, and at the boundary $\left(r=R_{0}\right)$ of the MFT the direction of the magnetic field vector $\boldsymbol{B}=\left(B_{r}, B_{z}\right)$ is given by the radial expansion of the tube (Fig. 4.1). Between the axis and the boundary we assume a linear behavior of the radial component

$$
B_{r}(r, z)=B_{z}(z) \frac{r}{R_{0}} \frac{\mathrm{d} R(z)}{\mathrm{d} z}
$$

The radial component $B_{r}(r, z)$, introduced in such a way, is the only deviation from the thin flux tube approximation. Other physical parameters of the internal atmosphere are functions of the height only. It can easily be seen that the adopted components $B_{r}$ and $B_{z}$ satisfy the condition $\operatorname{div} \boldsymbol{B}=0$. 
Thus, $R_{0}$ and $B_{0}$ are the two free parameters which fully define the geometry and magnetic field configuration of a single flux tube. Typical values of these parameters to be used in the model are $100 \mathrm{~km}$ and $1400 \mathrm{G}$, respectively. The corresponding magnetic flux then is $\Phi=4.4 \times 10^{17} \mathrm{Mx}$.

The dependence, defined by Eq. 4.3, implies infinite expansion of a tube with height. Yet, such a configuration leads to the intersection at certain heights with the fields of neighboring flux tubes. Yet it is generally believed that MFTs while expanding upwards are merging and forming a so called magnetic canopy. Pneuman et al. (1985) have derived a tube model in which they also considered the merging of flux tubes. In our case, in order to avoid ambiguity caused by the intersection of the MFTs we have restricted the expansion of the flux tube, introducing the maximum radius which it can reach (Fig. 4.1). The latter depends on the merging height, which in turn depends on the number density of MFTs on the surface, or number of tubes in the simulation box.

\subsubsection{Temperature stratification inside MFT}

An adequate choice of the temperature stratification inside flux tubes is an important aspect of the numerical modeling and radiative transfer calculations. Due to the partial evacuation of flux tubes and consequently reduced number of absorbers the radiation from magnetic elements always emerges from deeper layers of the MFT than outside, so that the level of the continuum optical depth $\tau_{\text {cont }}=1$ in magnetic atmospheres corresponds to lower geometrical heights than in the ambient plasma. The difference in heights of the continuum radiation formation between the magnetic and the non-magnetic photosphere is called Wilson depression and, for network flux tubes for example, it is usually of the order of $100 \mathrm{~km}$. Yet, for different temperature stratifications inside a flux tube this value can vary due to several thermal effects, such as change of ionization rates and of density. Obviously, temperature is a determinative factor in processes of the continuum radiation and spectral line formation. In this way, the temperature has an effect on many observed properties of facular structures, such as: CLV of the intensity contrast, continuum and line core contrasts, line depressions, and accordingly amplitudes of the polarimetric signal.

A realistic temperature model can be found either from a solution of a system of MHD equations, or by means of inversion of high resolution spectroscopic data observed in facular structures, or from the analysis of the CLV of the intensity contrast. In the forward modeling which is performed in this work the temperature stratification cannot be obtained consistently from some equations. It is a free parameter of the model, which should be prescribed independently from other model parameters. Therefore, we need to define a reasonable temperature as a function of height $T(z)$ inside a single magnetic flux tube.

It is generally accepted that strong magnetic fields inhibit convective energy transport, resulting in a reduced temperature inside a MFT at any geometrical height. Such a model is called hot wall and was first proposed by Spruit (1976) and later developed by Deinzer et al. (1984b). As it was mentioned in Sect. 3.1.1 the hot wall concept is often used to explain the observed CLV of the continuum brightness of plage faculae.

An alternative mechanism (hot cloud model) of the increased facular contrast at large heliocentric angles was suggested by Knölker et al. (1988). In the hot cloud model flux tubes are characterized by some temperature excess in the upper layers of the photosphere. 


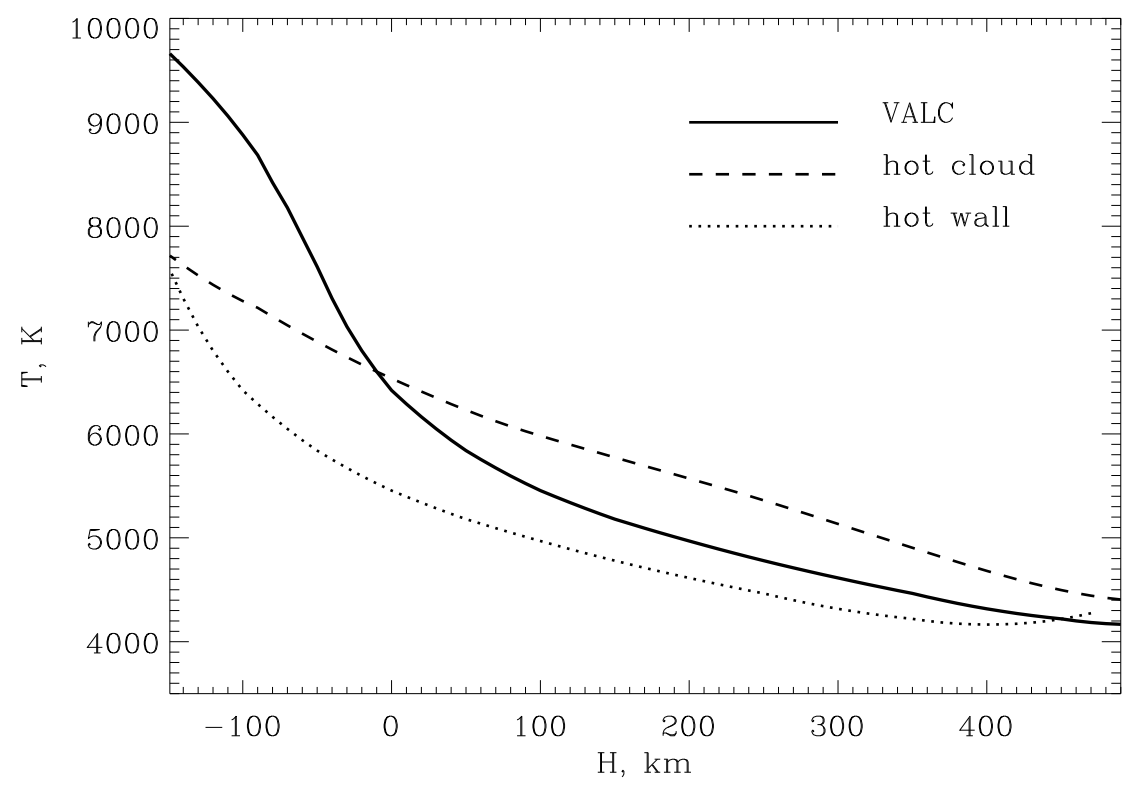

Figure 4.2: Three variants of the internal temperature stratifications used in numerical modeling.

This is due to the lateral influx of heat by horizontal radiative transfer in the medium with strong horizontal pressure gradients. It was shown by Fabiani Bendicho et al. (1992), who have performed 2D numerical radiative transfer calculations, that for thin flux tubes radiation channeling leads to an enhancement of the temperature of the upper layers. Later, Bellot Rubio et al. (2000) have found from the inversion of spectra from plage faculae, that flux tube atmospheres are cooler than the ambient gas at heights below $z=0$ $\mathrm{km}$ and hotter at $z>0$. Eker (2003) has presented a comprehensive study of various cloud models of solar faculae as plane parallel structures above the photosphere and their influence on the center-to-limb variations of the intensity contrast.

Both temperature models, hot wall and hot cloud, were adopted in our modeling as reference thermal stratifications. To construct a hot wall model with a flux tube cooler at all geometrical heights we have shifted $T(z)$, given by the VALC model, downward by a Wilson depression of $\Delta z=100 \mathrm{~km}$. The choice of the hot cloud stratification is arbitrary with cooler $T$ at $z<0 \mathrm{~km}$ and hotter $T$ at $z>0 \mathrm{~km}$. Additionally, to separate thermal effects from the effects of the reduced pressure inside MFTs, one more temperature model was used in the model calculations, the model with the same internal temperatures as outside at the same geometrical levels. The chosen curves as functions of the geometrical height in the solar photosphere are presented in Fig. 4.2.

The evident limitation of such an approach is that we keep the temperature of the ambient non-magnetic atmosphere unchanged, and assume the temperature from the average photospheric model described by the VALC model (Vernazza et al., 1981). Yet, it was shown by Hasan et al. (1998) that for the interpretation of the Stokes profiles from unresolved magnetic elements one should consider both internal and external temperatures, and that such a treatment results in an external temperature different from the one 
given by average models. Another point is that we adopt only the three above temperature stratifications inside a MFT and keep them fixed, in other words we do not adjust them iteratively as it is done with an inversion technique. Therefore, no perfect correspondence to the shape of the observed intensity profiles is expected. As it was already mentioned the aim of such an approach is to test the principle ability of several models to reproduce averaged observed properties of polar faculae.

\subsubsection{Calculation of the atmospheric parameters}

The atmosphere of each of the two components of the model (internal end external) is described by gas pressure and temperature stratifications $\left(P_{g}, T\right)$ as functions of geometrical scale $z$. However, to solve the radiative transfer equation, we need to know for each component several physical quantities such as continuum opacity, which requires the knowledge of the electron pressure, the partial hydrogen pressure, and the line opacity, i.e. the number density of the absorbers. All model calculations were made under the assumption of local thermodynamical equilibrium LTE:

* the Maxwell distribution with the same kinetic temperature for all species was assumed (particularly, $T_{e}=T$ );

* the Boltzmann and Saha formulae were used to calculate the atomic level populations and the ionization equilibria, respectively;

* the continuum source function depends only on the local temperature and is equal to the Planck function $S_{c}=B_{\lambda}(T)$;

* the line core source function is equal to the continuum source function $S_{l}=S_{c}$.

The electron pressure was calculated as a function of gas pressure and temperature $P_{e}=f\left(P_{g}, T\right)$, following the recipes of Aller (1963). We have taken into account the electron contribution only from those chemical elements which are most abundant in the solar photosphere and simultaneously are good electron donors, i.e. have low ionization energies. Contributions from 16 chemical elements were considered: $\mathrm{H}, \mathrm{He}, \mathrm{C}, \mathrm{O}, \mathrm{N}$, $\mathrm{Na}, \mathrm{Mg}, \mathrm{Al}, \mathrm{Si}, \mathrm{S}, \mathrm{K}, \mathrm{Ca}, \mathrm{Cr}, \mathrm{Mn}, \mathrm{Fe}, \mathrm{Ni}$. The first and the second ionization states were taken into account. So, the number of neutral atoms and ions of each element $z$ is $N_{z}=N_{I}+N_{I I}+N_{I I I}$. Combining the latter with the Saha equation we introduce ionization ratios:

$$
f_{I I}=\frac{N_{I I}}{N_{z}}=f_{I I}\left(P_{e}, T\right), \quad f_{I I I}=\frac{N_{I I I}}{N_{z}}=f_{I I I}\left(P_{e}, T\right),
$$

The number of free electrons per nucleus is given by

$$
E=\frac{\sum_{z} N_{z} f_{I I}(z)+2 \sum_{z} N_{z} f_{I I I}(z)}{\sum_{z} N_{z}}
$$

Then we construct the ratio of the total gas pressure to the electron pressure, which gives the sought relation

$$
\frac{P}{P_{e}}=\frac{\left(N_{\text {ions }}+N_{\text {atoms }}+N_{e}\right) k T}{N_{e} k T}=\frac{N_{\text {nuclei }}+N_{e}}{N_{e}}=\frac{E+1}{E}
$$


However, this equation can not be used directly to find $P_{e}$ for given $P_{g}$, since $E$ itself is a function of the electron pressure, but it can be solved iteratively. Starting with the guess value $P_{e}^{0}$ and calculating the next approximation $P_{e}^{1}$, we proceed in such a way with iterations until the required precision $\epsilon$ is reached $\left|P_{e}^{n}-P_{e}^{n-1}\right|<\epsilon$.

$$
P_{e}=P_{e}^{0} \quad \rightarrow \quad P_{e}^{1}=P \frac{E}{E+1} \quad \rightarrow \quad \cdots
$$

The partial hydrogen pressure can be found as a function of temperature, gas and electron pressure $P_{\mathrm{H}}=f\left(P_{g}, P_{e}, T\right)$. Neglecting the contribution of $\mathrm{H}^{-}$, ionized helium and metals whose abundances are negligible compared to the abundances of hydrogen and helium we can write (Landi Degl'Innocenti 1976)

$$
P-P_{e}=P_{\mathrm{H}}+P_{\mathrm{H}^{+}}+P_{\mathrm{He}}=\left(P_{\mathrm{H}}+P_{\mathrm{H}^{+}}\right)\left(1+A_{\mathrm{He}}\right)
$$

After simple algebra we find for the partial hydrogen pressure

$$
P_{\mathrm{H}}=\frac{P-P_{e}}{\left(1+A_{\mathrm{He}}\right)(1+q)}, \quad q=\frac{P_{\mathrm{H}^{+}}}{P_{\mathrm{H}}},
$$

where $A_{\mathrm{He}}$ is the helium abundance and $q$ can be found from the Saha equation.

The continuous absorption coefficient was calculated using numerical formulae given by Landi Degl'Innocenti (1976). It was corrected for stimulated emission and includes contributions from bound-free and free-free transitions of $\mathrm{H}^{-}$, photoionization of neutral hydrogen, Rayleigh scattering on hydrogen atoms and Thompson scattering on free electrons.

The line absorption coefficient requires the knowledge of the number of absorbers in the lower atomic level involved in the transition, which is calculated for each spectral line, combining the Saha and the Boltzmann equations. For example, for neutral iron with abundance $A_{\mathrm{Fe}}$ we have

$$
N_{\text {Low }}=A_{\mathrm{Fe}} \frac{N_{\text {Low }}}{N_{\mathrm{Fe} \text { I }}} \frac{N_{\mathrm{Fe} \text { I }}}{\left(N_{\mathrm{Fe} \text { I }}+N_{\mathrm{Fe} \text { II }}\right)}\left(N_{\mathrm{H}}+N_{\mathrm{H}^{+}}\right)
$$

Only the first ionization state, with the ionization energy $\chi_{1}=7.87 \mathrm{eV}$ of neutral iron, was taken into account. Due to the relatively low temperatures in the photosphere and high second ionization energy $\left(\chi_{2}=16.81 \mathrm{eV}\right)$ only a very small fraction of iron atoms is ionized twice. For the calculations of the number density of absorbers we used the iron abundance of $\left(\lg A_{\mathrm{Fe}}+12\right)=7.5$.

The other quantities such as damping constant $\Gamma$, Doppler broadening $\Delta \lambda_{D}$, FaradayVoigt functions and the Planck function $B_{\lambda}(T)$ were calculated in the usual way (Rees et al. 1989, Landi Degl'Innocenti, 1976). The Doppler broadening reads in evident notation

$$
\Delta \lambda_{D}=\frac{\lambda}{c} \sqrt{\frac{2 k T}{M_{a}}+\xi^{2}}
$$

where $\xi$, a microturbulent velocity, is an additional mechanism of line broadening. For the model calculations we adopt the microturbulent velocity from the VALC model for both external and internal atmosphere. 


\subsubsection{Construction of the 3D box and multiple rays}

After the model of one single MFT is established and the parameters of the photosphere which enter the radiative transfer equation are calculated for both components of the model (magnetic and non-magnetic), the next step of the numerical modeling is an accurate treatment of the geometrical aspect of the problem. A full 3D model was constructed with the vertical size $Z_{s}$, given by the range of heights of the photospheric model, and with the horizontal extent of the box $X_{s}=3^{\prime \prime}$ and $Y_{s}=2^{\prime \prime}$, where the $Y$ axis is parallel to the limb and structures in this direction will not exhibit perspective foreshortening close to the limb, and the $X$ axis is perpendicular to the limb. The chosen size of the simulation box was inspired by the observed facular shapes and sizes (Sect. 3.1.2). It was found that PFs are often organized in chains with few $\operatorname{arcsec}\left(2^{\prime \prime}\right.$ in our model) lengths in $Y$ direction, and with smaller sizes of about $1^{\prime \prime}$ at $\mu \sim 0.3$ in the direction perpendicular to the limb. Accounting for the limb effects the chosen size of the simulation box approximately correspond to the observed sizes of PFs.

Next, the simulation box was populated with identical MFTs randomly distributed on the surface (XY plane at $z=0 \mathrm{~km}$ ). To study the effect of many flux tubes along the line-of-sight at large heliocentric angles, we have used three realizations of the MFTs distribution in the simulation box (hereafter referred to as distribution $\mathrm{N} 1, \mathrm{~N} 2$ and N3), which differ only in the number of MFTs in the box (Fig. 4.3). The distribution N1 has only 5 MFTs in the $3^{\prime \prime} \times 2^{\prime \prime}$ box, the distributions N2 and N3 accordingly have 11 and 21 flux tubes in the box. It is more customary to describe the number density of MFTs in terms of a filling factor, analogously to the observational $f_{\text {obs }}$. We introduce the filling factor of the model $f_{\text {mod }}$ defined as the ratio of the area occupied by the flux tubes at $z=0 \mathrm{~km}$ to the total area of the simulation box:

$$
f_{\mathrm{mod}}=\frac{\pi R_{0}^{2} N_{\mathrm{MFT}}}{S_{\text {box }}}
$$

Assuming $R_{0}=100 \mathrm{~km}$ we obtain for the three adopted distributions of MFTs $f_{\text {mod }}(N 1)=0.05$, $f_{\text {mod }}(N 2)=0.1$ and $f_{\text {mod }}(N 3)=0.2$. We note, that the thus introduced $f_{\text {mod }}$ should not be con-

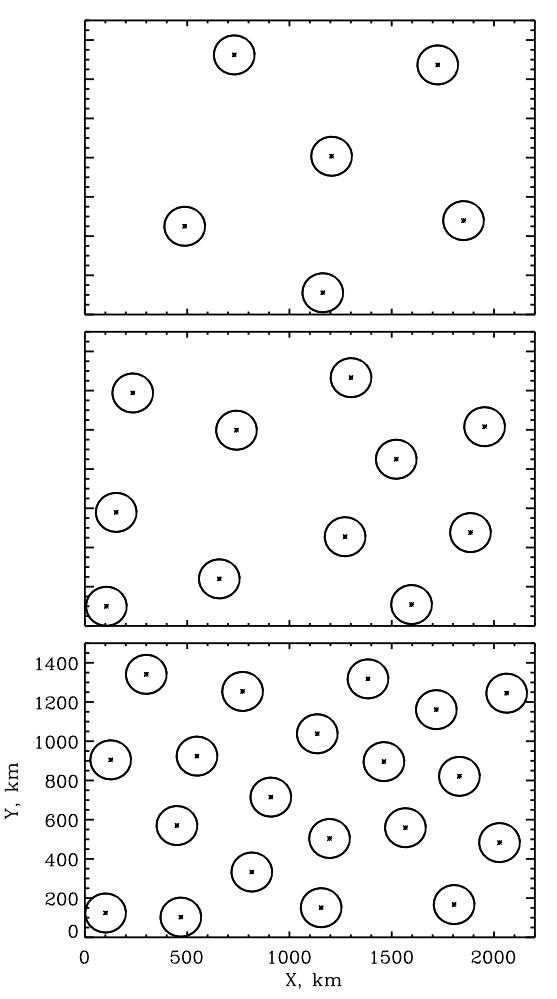

Figure 4.3: Three random distributions of flux tub es in the simulation box. Radius $R_{0}=100 \mathrm{~km}$. fused with the usual $f_{\text {obs }}$. In the process of the numerical modeling the free parameter $R_{0}$ can be changed, the synthetic intensity maps will be convolved with smearing functions to simulate limited spatial resolution of the observations. All these result in changes of the true filling factor. Moreover, for the observations and modeling of facular structures at large heliocentric angles the concept of filling factor $f_{\text {obs }}$ looses its original meaning. At the limb the magnetic field is inhomogeneous not only in the direction perpendicular to the LOS but also, what is even more important, is highly intermittent in the direction along the LOS. This effect can not be described in terms of a filling factor. 


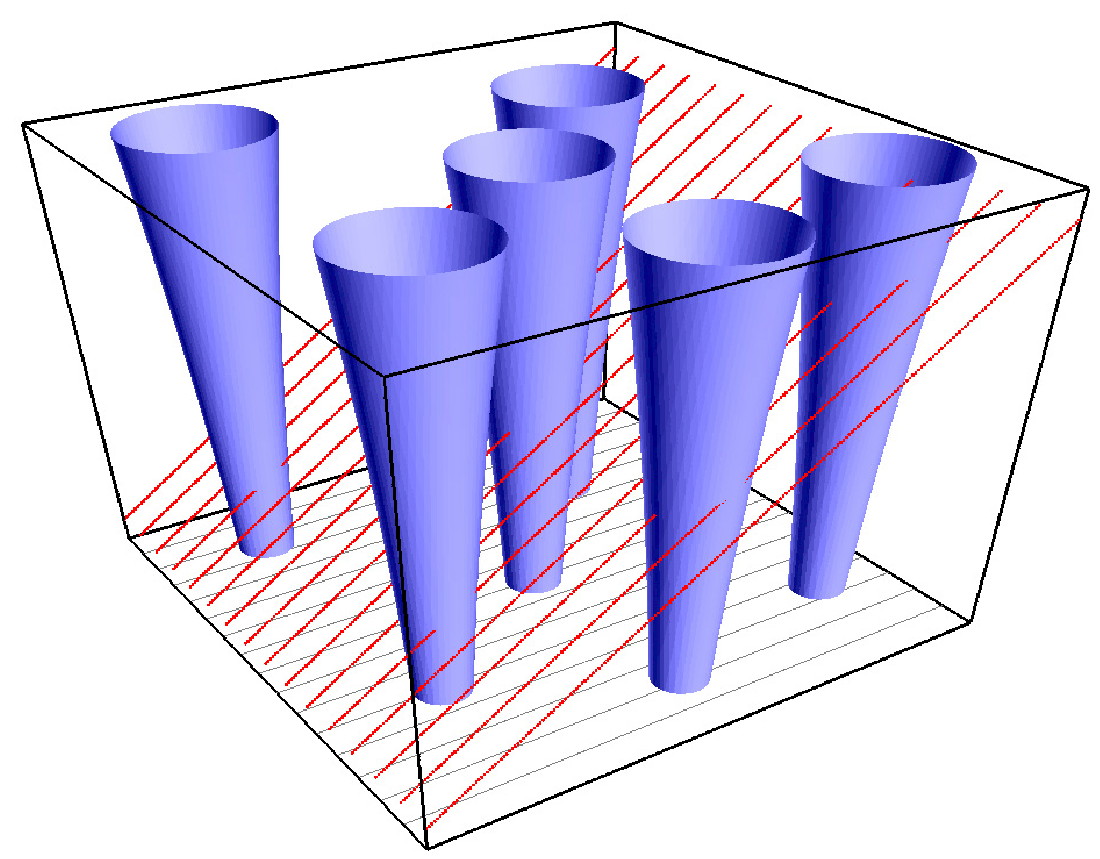

Figure 4.4: 3D simulation box populated with isolated MFTs crossed by an array of parallel rays, schematically.

In such a way, our 3D two-component model (for a given set of free parameters of the model: $R_{0}, B_{0}$, distribution of MFTs on the surface and internal thermal stratification) is fully described by:

- external atmosphere with all parameters, required by the radiative transfer equation and depending only on height, and with $\boldsymbol{B}=0$;

- internal atmosphere with $\boldsymbol{B}(z, r) \neq 0$, and with defined stratifications of the other parameters;

- coordinates of the centers of the $N$ magnetic flux tubes $\left(x_{c}^{i}, y_{c}^{i}\right)$, where $i=1 \ldots N$.

For the thus constructed 3D simulation box a radiative transfer problem was solved for various inclinations of the line-of-sight to the vertical direction $Z$, corresponding to observations at different heliocentric angles. To obtain the center-to-limb variation the calculations were performed at several inclination $\mu=\cos \theta=(1 ., 0.8,0.6,0.4,0.2,0.1)$. For each $\mu$ an array of parallel rays was introduced along which the integrations were performed as shown in Fig. 4.4. Each ray crosses several ingomogeneities along the LOS resulting from the non plane-parallel atmosphere of the 3D box, but the RT calculations are performed separately for each ray and independently from the other rays. Such an approach is called $1.5 \mathrm{D}$ radiative transfer.

The detailed geometry of each ray passing the flux tubes was considered exactly:

- the rays do not necessarily pass through the axis of the MFTs (Fig. 4.5a);

- the rays cross the MFTs at various heights in the photosphere (Fig. 4.5b);

- the angle $\gamma$ between the LOS and $\boldsymbol{B}$ and the azimuthal angle $\chi$ are taken into account exactly, they depend on the height and distance from the axis (Fig. 4.5c). 


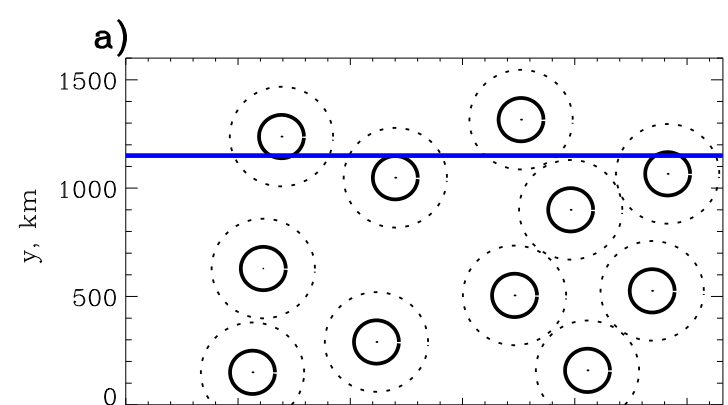

c)

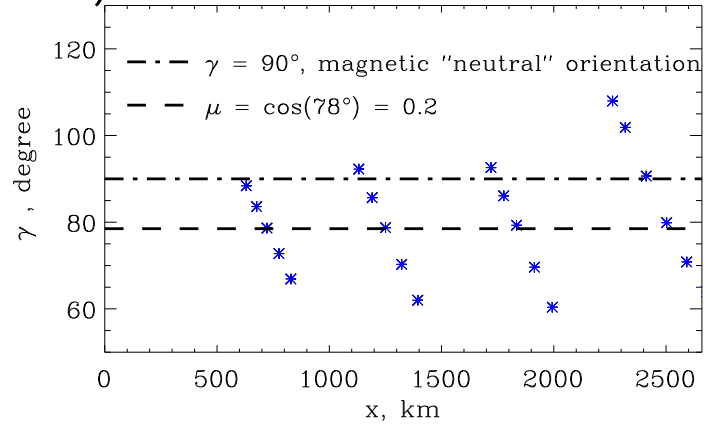

b)

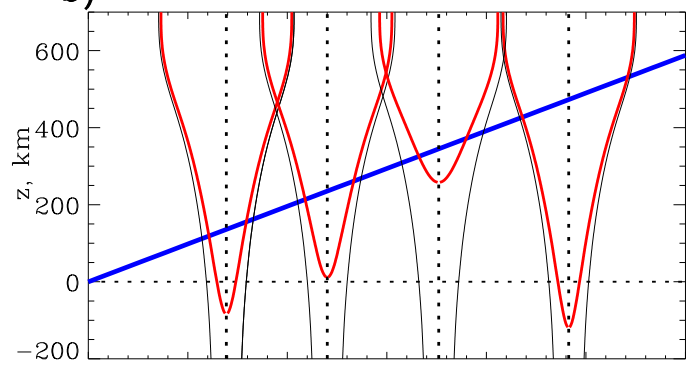

d)

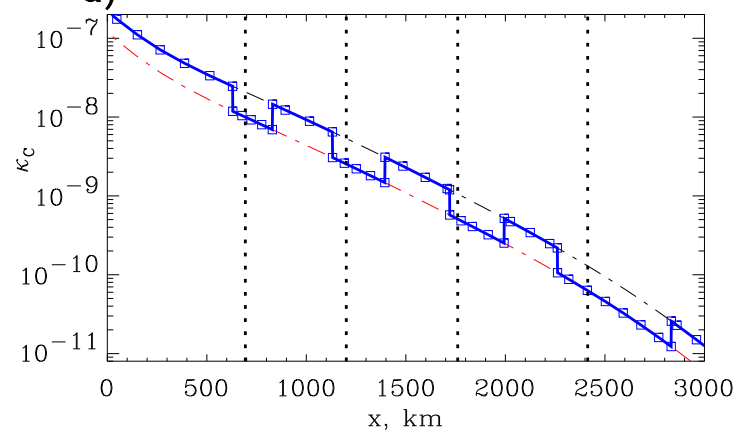

Figure 4.5: Geometry of a ray crossing several flux tubes at $\mu=0.2$ in the X-Y plane (a) and the X-Z plane (b). Panel c: angle between $\boldsymbol{B}$ and LOS as a function of spatial position; dashed: for purely vertical magnetic fields; dashed-dotted: magnetic field oriented perpendicular to the LOS; asterisks: inclination angles at grid points inside the MFTs. Panel d: continuum opacity $\kappa_{C}$ along the integration path (solid blue line) consisting of $\kappa_{C}^{i n t}$ (red dotted dashed line) on the path through the MFTs and of $\kappa_{C}^{e x t}$ (black dashed line) elsewhere; vertical dotted lines show the locations of the flux tube centers (b).

We note the importance of the exact treatment of the angles for the realistic modeling. The geometrical path of the ray through the MFT can be divided into two parts: from the entrance to the MFT to the center and from the center to the exit (Fig. 4.5b). The contribution to the Stokes $V$ signal is different for both parts. The second part is geometrically longer than the first and with the inclination $\gamma$ smaller than the heliocentric angle $\theta$ resulting in a stronger contribution to the Stokes $V$. Yet, the first part of the path is short but can have a 'neutral line' (no contribution to $V$ ) and even can have a negative contribution to the emergent $V$ signal $\left(\gamma>90^{\circ}\right)$ (Fig. 4.5c). Moreover, numerical tests have shown that for strongly inclined magnetic fields (MFTs at the limb) due to the magneto-optical effects the Stokes $V$ signal also depends strongly on the azimuthal angle $\chi$.

To provide a satisfactory precision of the integration an irregular adaptive grid along each ray was used and an optical step size $\Delta l g(\tau)=0.1$ was not exceeded. To account for the contribution of optically thin MFTs to the integration, flux tubes contain at least five grid points (Fig. 4.5c-d). The physical conditions inside a MFT differ strongly from that in the ambient plasma. This leads in the numerical model to an abrupt jump of the integrand on the boundary of MFTs. For a sufficiently accurate treatment of the jump two additional grid points were introduced at the boundary, separated by a very small distance, which gives negligible contribution to the integration (Fig. $4.5 \mathrm{~d}$ )

The spacing between the rays is $25 \mathrm{~km}\left(\sim 00^{\prime \prime} 035\right)$ in both $X$ and $Y$ directions. Such a high resolution of the model was provided at the expense of high computational demands. 
For the adopted size of the simulation box of $3^{\prime \prime} \times 2^{\prime \prime}$ such a resolution requires approximately 6000 individual rays at $\mu=\cos \theta=1.0$ and even more at larger heliocentric angles $\theta$. The rays which are strongly inclined to the vertical direction require a larger horizontal extent of the simulation box to account for the increased geometrical path of the rays along the line-of-sight.

\subsection{Radiative transfer in a magnetized atmosphere}

The broadening of spectral lines in the presence of a magnetic field was for the first time observed in laboratory conditions by Zeeman in 1897. Since the first astrophysical application of the Zeeman effect in 1908 by Hale, who measured the splitting of spectral lines emerging from active regions of the Sun and thus proved that sunspots possess kilo-Gauss fields, it has become a main tool for studying magnetic fields of stars. The pronounced polarization signature of the split components makes this diagnostic especially useful for weak and/or unresolved magnetic structures on the Sun. Nowadays, the theory of spectral line formation in the presence of magnetic fields is well established (Unno 1956, Rachkovksy 1962). Numerical recipes for the radiative transfer calculations of polarized light in plane parallel atmospheres under the assumption of LTE have been developed and successfully applied since several decades for spectral line synthesis and for the interpretation of polarimetric observations of solar magnetic fields (Wittmann 1974, Landi Degl'Innocenti 1976, Rees et al. 1989).

Radiative transfer calculations in LTE rely on the assumption that the physical conditions of the plasma in each point are "disconnected" from the radiation field and depend only on the local thermodynamical properties of the gas, which are established by the collisions. The LTE assumption is best fulfilled in the lower levels of the photosphere where the density is relatively high and the collisions control the energy distribution. The spectral lines used in this work originate from the heights where the LTE assumption is valid, except for the region of the line core formation of the strong Fe I 6301.5 line. The latter is formed in the upper layers of the solar photosphere $(z \sim 500 \mathrm{~km})$ where NLTE effects become significant (Shchukina \& Trujillo Bueno 2001). However, in the modeling performed for this study all NLTE effects were neglected.

Extensive reviews on radiative transfer of polarized light and related problems can be found for example in the lecture notes "Radiative Transfer in Stellar Atmospheres" by Rutten (available in the internet at http://www.astro.uu.nl/ rutten/node21.html) and in Landi Degl'Innocenti (1992). In the following sections we briefly introduce the concepts and equations using as examples the three iron lines which were observed in this study. Their atomic data are listed in Table 4.1, where $g$ denotes the Landé factor, $g_{w}$ the statistical weight of the lower level and $f$ the oscillator strength (Thévenin 1990).

\subsubsection{Zeeman effect and Stokes vector}

Atomic levels are characterized by the set of quantum numbers $S, L$ and $J$, describing spin, orbital angular momentum and the total angular momentum of the atom. Two states which differ at least in one quantum number will have different energies. The transition between two such atomic levels (hereafter referred to as upper and lower levels with 
Table 4.1: Configuration of transitions

\begin{tabular}{c|c|ccc|ccc|c|c} 
& $\begin{array}{c}E_{p}, \\
\mathrm{eV}\end{array}$ & \multicolumn{3}{|c|}{ Lower level } & \multicolumn{3}{|c|}{ Upper level } & & \multirow{2}{*}{ Term } \\
\hline & $S L J$ & $g$ & Term & $S L J$ & $g$ & $g_{\text {eff }}$ & $\lg \left(g_{w} f\right)$ \\
\hline 6301.5 & 3.65 & $z^{5} P$ & 212 & 1.83 & $e^{5} D$ & 222 & 1.5 & 1.67 & -0.59 \\
6302.5 & 3.69 & $z^{5} P$ & 211 & 2.5 & $e^{5} D$ & 220 & 0 & 2.5 & -1.16 \\
6149.3 & 3.65 & $b^{4} D$ & 1.520 .5 & 1.33 & $z^{4} P$ & 1.510 .5 & 2.67 & 1.33 & -2.88
\end{tabular}

corresponding quantum numbers $J_{u}$ and $J_{l}$ ) is accompanied by the absorption/emission of a photon with a wavelength $\lambda=h c /\left(E_{u}-E_{l}\right)$, where $E_{u}$ and $E_{l}$ are the energies of the atomic levels involved in the transition, $h$ and $c$ are the Planck constant and speed of light, respectively.

Each level has one more quantum number $M$, defined as a projection of the total angular momentum on a reference direction. Under normal conditions atomic levels are degenerate with respect to this quantum number: states with different values of $M$ have the same energy. However, the magnetic field removes this degeneracy, splitting one level into $2 J+1$ sublevels with magnetic quantum number $M=-J \ldots+J$ and possessing different energies. In the presence of a magnetic field, $M$ is a projection of $J$ on the direction of the magnetic field. The transitions will now occur following the selection rule $\Delta M=-1,0,1$. All transitions between sublevels with $\Delta M=0$ are called $\pi$ components and with $\Delta M= \pm 1$ are the $\sigma$ components.

A simple configuration of a Zeeman splitting is a triplet with $J_{u}=0$ and $J_{l}=1$. An example of such a triplet is the Fe I $6302.5 \AA$ line (Fig. 4.6 left panel). The lower level is split into $(2 J+1)=3$ sublevels which together with the magnetically insensitive upper level produce three spectral lines: the central unshifted $\pi$ component and two $\sigma$ components. The amount of the splitting $\Delta \lambda_{B}$ is proportional to the magnetic field and the Landé factor

$$
\Delta \lambda_{B}=\lambda-\lambda_{0}=\frac{e}{4 \pi c m_{e}} g^{*} \lambda^{2} B
$$

where $\lambda_{0}$ is the central wavelength, $\lambda$ is the wavelength of the shifted component and $g^{*}$ is the Lande factor of the transition, which in case of the triplet is equal to the Lande factor $g$ of a level, a combination of quantum numbers of the split level, which characterizes the magnetic sensitivity of the level

$$
g=1+\frac{J(J+1)+S(S+1)-L(L+1)}{2 J(J+1)}
$$

The normalized strengths of the split components were calculated following Landi Degl'Innocenti (1976). In case of the Zeeman triplet the strengths of the $\pi$ component and two $\sigma$ components are equal to unity (Fig. 4.6 left panel). 

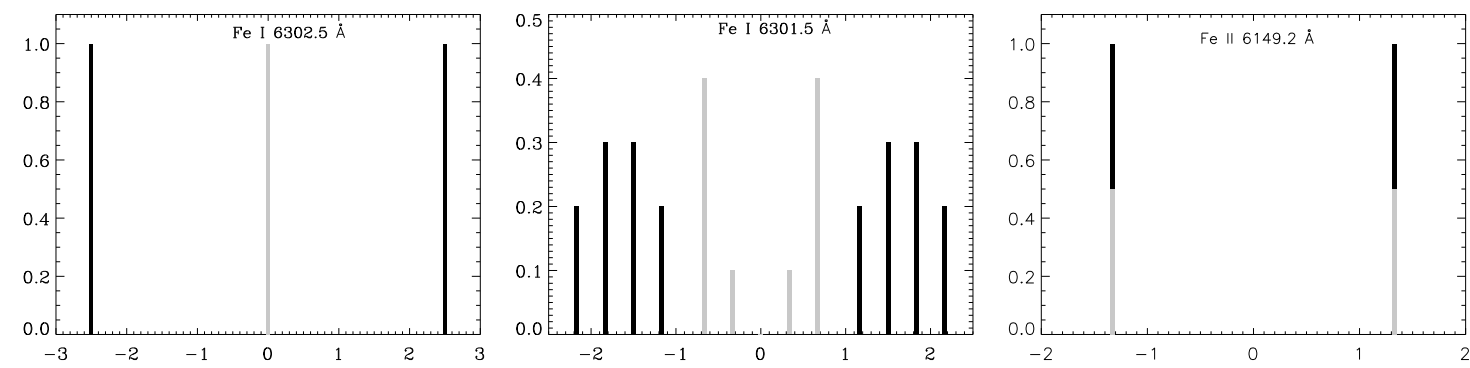

Figure 4.6: Configuration of the Zeeman splitting of the three iron lines with $\pi$ components as gray lines and $\sigma$ components as black lines, the abscissa is in units of the Landé factor, and the ordinate gives the normalized strengths.

The spectral line Fe I $6301.5 \AA$ possesses a more complicated Zeeman pattern in the presence of a magnetic field. It belongs to the same multiplet as Fe I $6302.5 \AA$, but has $J_{u}=2$ and $J_{l}=2$, so that each level splits into $(2 J+1)=5$ sublevels. All transitions between these sublevels allowed by the selection rules form the Zeeman pattern, are known as anomalous Zeeman splitting. It is shown on the middle panel of Fig. 4.6. In this case the factor $g^{*}$ from Eq. 4.13 is given by

$$
g^{*}=g_{l} M_{l}-g_{u} M_{u}
$$

An even more exotic splitting exhibits the transition Fe II $6149.3 \AA$ with $J_{u}=0.5$ and $J_{l}=0.5$ and correspondingly 2 sublevels for both upper and lower atomic levels. In this case the $\pi$ component consists of two transitions, and each of them coincides with the blue and the red $\sigma$ components (Fig. 4.6 right panel).

A remarkable peculiarity of the Zeeman effect, which makes it especially useful for the study of small-scale structures on the Sun, is a pronounced polarimetric signature. When the line-of-sight coincides with the direction of the magnetic field (longitudinal case) spectral lines which belong to $\sigma$ components are circularly polarized, and the $\pi$ component is not visible. When observing perpendicularly to the magnetic field (the transverse case) all components are visible. In absorption, the $\pi$ component is linearly polarized in the direction perpendicular to the magnetic field $\boldsymbol{B}$ and the $\sigma$ components are linearly polarized parallel to $\boldsymbol{B}$.

Polarized light is completely described by the 4-component Stokes vector

$$
\boldsymbol{I}=(I, Q, U, V)^{T}
$$

where $T$ means transposition, $I$ is the total intensity, and the other three components are defined as:

$$
\begin{gathered}
Q=I_{\text {lin }}\left(0^{\circ}\right)-I_{\text {lin }}\left(90^{\circ}\right) \\
U=I_{\text {lin }}\left(45^{\circ}\right)-I_{\text {lin }}\left(135^{\circ}\right) \\
V=I_{\text {circ }}^{+}-I_{\text {circ }}^{-}
\end{gathered}
$$

where $I_{\text {circ }}^{+}$and $I_{\text {circ }}^{-}$stand for left and right circularly polarized light and $I_{\text {lin }}(\phi)$ for linearly polarized light with a polarization plane rotated by an angle $\phi$ relative to some reference direction. 


\subsubsection{Radiative transfer equation and numerical solution}

The problem of radiative transfer (RT) can be formulated as the determination of the Stokes vector for given stratification of the physical parameters in the atmosphere. The radiative transfer equation (RTE) for the Stokes vector is

$$
\frac{\mathrm{d} \boldsymbol{I}}{\mathrm{d} z}=-\mathbf{K} \boldsymbol{I}+\boldsymbol{j}
$$

where the total absorption matrix $\mathbf{K}$ and the total emission vector $\boldsymbol{j}$ are given by

$$
\begin{gathered}
\mathbf{K}=\kappa_{c} \mathbf{1}+\kappa_{0} \boldsymbol{\Phi} \\
\boldsymbol{j}=\kappa_{c} S_{c}+\kappa_{0} S_{l} \boldsymbol{\Phi}
\end{gathered}
$$

The line absorption matrix $\Phi$ has only 7 independent elements:

$$
\boldsymbol{\Phi}=\left(\begin{array}{cccc}
\eta_{I} & \eta_{Q} & \eta_{U} & \eta_{V} \\
\eta_{Q} & \eta_{I} & \rho_{V} & -\rho_{U} \\
\eta_{U} & -\rho_{V} & \eta_{I} & -\rho_{Q} \\
\eta_{V} & \rho_{U} & -\rho_{Q} & \eta_{I}
\end{array}\right)
$$

where

$$
\begin{gathered}
\eta_{I}=\frac{1}{2} \phi_{p}+\frac{1}{4}\left(\phi_{r}+\phi_{b}\right)\left(1+\cos ^{2} \gamma\right) \\
\eta_{Q}=\frac{1}{2}\left[\phi_{p}-\frac{1}{2}\left(\phi_{r}+\phi_{b}\right)\right] \sin ^{2} \gamma \cos 2 \chi \\
\eta_{U}=\frac{1}{2}\left[\phi_{p}-\frac{1}{2}\left(\phi_{r}+\phi_{b}\right)\right] \sin ^{2} \gamma \sin 2 \chi \\
\eta_{V}=\frac{1}{2}\left(\phi_{r}-\phi_{b}\right) \cos \gamma \\
\rho_{Q}=\frac{1}{2}\left[\phi_{p}^{\prime}-\frac{1}{2}\left(\phi_{r}^{\prime}+\phi_{b}^{\prime}\right)\right] \sin ^{2} \gamma \cos 2 \chi \\
\rho_{U}=\frac{1}{2}\left[\phi_{p}^{\prime}-\frac{1}{2}\left(\phi_{r}^{\prime}+\phi_{b}^{\prime}\right)\right] \sin ^{2} \gamma \sin 2 \chi \\
\rho_{V}=\frac{1}{2}\left(\phi_{r}^{\prime}-\phi_{b}^{\prime}\right) \cos \gamma
\end{gathered}
$$

where $\phi$ and $\phi^{\prime}$ are the absorption and the dispersion profile, respectively, and the subscript $p$ refers to the $\pi$ component and $b$ and $r$ to the blue and the red $\sigma$ component, respectively.

For the peculiar Zeeman splitting of the Fe II $6149.3 \AA$ line with the $\pi$ components coinciding with the $\sigma$ components and having each half of the strengths of the latter (Fig. 4.6) we have $\left[\phi_{p}-\frac{1}{2}\left(\phi_{r}+\phi_{b}\right)\right]=0$ and $\left[\phi_{p}^{\prime}-\frac{1}{2}\left(\phi_{r}^{\prime}+\phi_{b}^{\prime}\right)\right]=0$, and therefore $\eta_{Q}=\eta_{U}=\rho_{Q}=\rho_{U}=0$ and accordingly $Q=U=0$. This transition does not produce linearly polarized components and thus does not suffer from $(Q, U) \rightarrow V$ crosstalk.

For the numerical integration of Eq. 4.17 we used the DELO (Diagolal Element Lambda Operator) method by Rees at al. (1989) with a linear interpolation between grid points. This numerical scheme provides a fast and accurate solution of the RTE (Eq. 4.17). The DELO method was programmed and its accuracy was tested by computing the intensities for cases in which an analytical solution of the transfer equation is known. 


\subsubsection{Spectral line synthesis and contribution functions}

The spectral lines under study were synthesized by solving the RTE along rays through the inhomogeneous model atmospheres at different wavelength positions. The atomic characteristic of the transitions listed in Table 4.1 were used for the spectral synthesis. The additional broadening of the intensity profiles caused by the macroturbulence was performed in a usual way by the convolution of synthetic line profiles with a Gaussian function of a width given by the macroturbulence velocity $v_{m a c}$. For our calculations we used the value of $v_{m a c}=1.25 \mathrm{~km} / \mathrm{s}$.

In order to calibrate our numerical model and to test the accuracy of the RT code and spectral line synthesis, we have calculated Stokes $I$ profiles from the average photospheric model VALC (Vernazza et al. 1981) and have compared them with the averaged observed profiles from the Fourier Transform Spectrometer (FTS) Atlas by Brault \& Neckel (cited by Neckel 1999). A satisfactory fit was achieved (Fig. 4.7). Similar to Pérez Rodríguez and Kneer (2002) we found that in order to fit the wings of the synthetic spectral lines to the reference ones some enhancement factor to the damping constant $C_{6}$ (Unsöld 1955) in the range of $2-5$ is required.
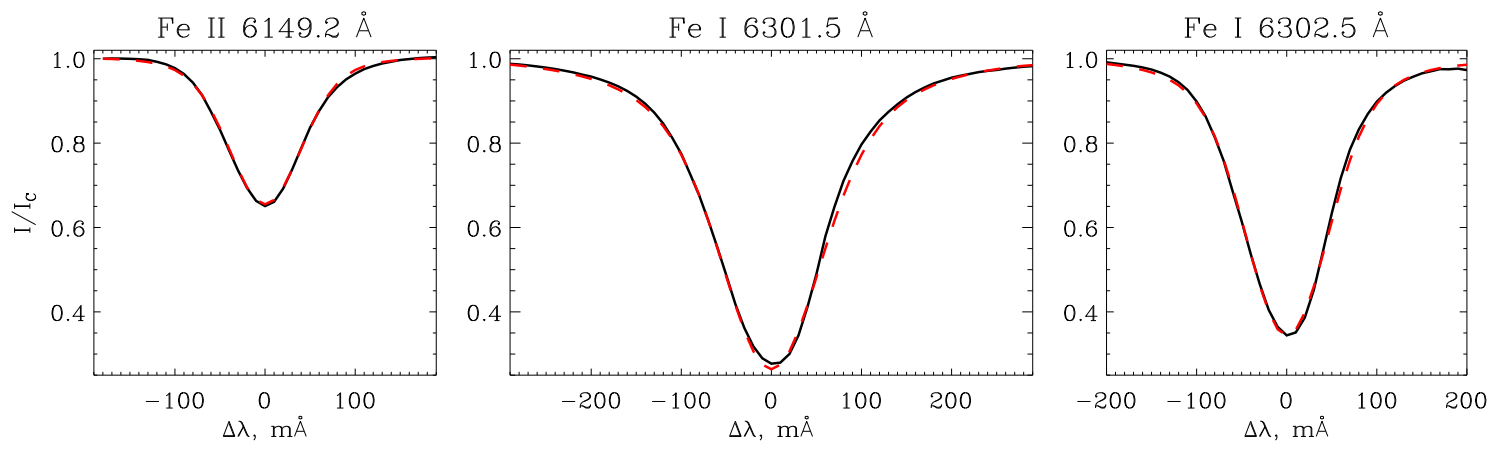

Figure 4.7: Comparison between calculated spectral lines (red dashed lines) and averaged observed lines form the high resolution FTS Atlas (black solid lines).

The advantage of the DELO method is that apart of the sought Stokes vector it allows to obtain contribution functions $\mathbf{C F}(z, \lambda)$ for a given atmosphere. This function shows the contribution of the different layers in the reference atmosphere to each of the four Stokes parameters and it can be used for the determination of the heights from where the spectral line emerges. The contribution function to the emergent intensity is defined as the integrand in the equation

$$
\boldsymbol{I}(\boldsymbol{\lambda})=\int_{-\infty}^{+\infty} \mathbf{C F}_{\mathbf{I}}(z, \lambda) \mathrm{d} z
$$

where integration is performed along the line-of-sight. For calculations at disk center $(\mu=1.0)$ the LOS coincides with the vertical axis $z$ of the model.

Defined in such a way $\mathbf{C F}_{\mathbf{I}}$ shows the contribution to the specific intensity at wavelength $\lambda$ in the LOS direction. However, it was pointed by Gurtovenko et al. (1974) and Magain (1986) that when we study the formation of spectral lines it is important to distinguish between heights of formation of the emergent intensity and the regions where the line depression is formed. 
The line depression of the Stokes $I$ profile is defined as

$$
R(\lambda)=\frac{I_{C}-I(\lambda)}{I_{C}}
$$

where $I_{C}$ is the continuum intensity near the spectral line. Magain (1986) and Rees et al. (1989) have derived a rigorous expression for the contribution functions to the line depression $\mathrm{CF}_{R}$ by rewriting the RTE for the line depression $R$ in form of Eq. 4.17, and then performing the same procedure as for the contribution function to the emergent intensity. $\mathrm{CF}_{R}$ defines precisely the region of the spectral line formation for a given atmosphere. Hereafter we will always use for short the notation $\mathrm{CF}$ for the contribution functions to the Stokes $I$ depression $\mathrm{CF}_{R}$.

When plotted as 2D maps in the $\lambda-z$ plane, CF gives a pictorial representation of the spectral line formation. As examples, the contribution functions calculated from the average photospheric model (VALC) at $\mu=1.0$ for the three iron lines under consideration are presented in Fig. 4.8.
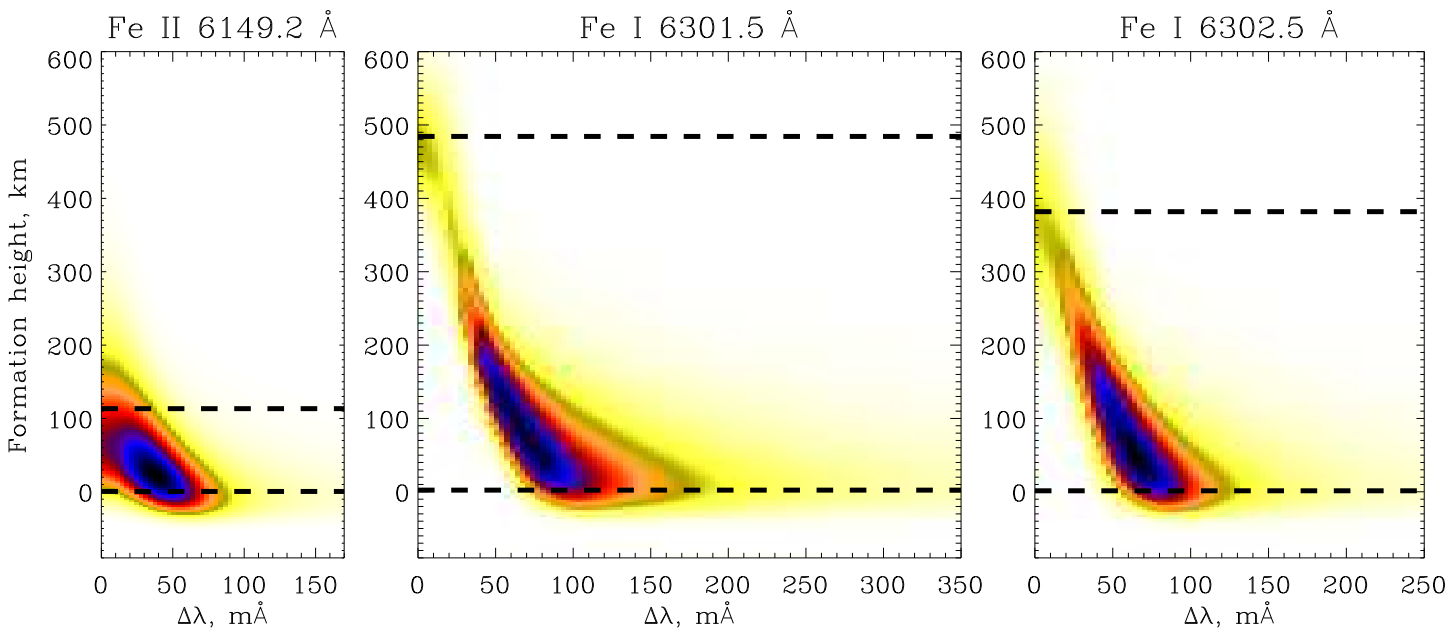

Figure 4.8: Contribution functions to the spectral line depression, calculated for three iron lines. The dashed lines show the range of heights where the spectral lines are formed. The corresponding heights were calculated as center of gravity of the contribution functions in the line core $(\Delta \lambda=0 \mathrm{~m} \AA)$ and in the continuum.

Due to the vertical gradients of the physical parameters in the solar photosphere the contribution functions for each spectral position are not symmetric in height; they have wings extended to the upper layers. Therefore the position of the maxima of the corresponding $\mathrm{CF}_{\lambda}$ underestimates the effective height of the line formation. A more accurate estimation can be found from the center-of-gravity (COG) of the corresponding $\mathrm{CF}$

$$
<z_{\lambda}>=\frac{\int_{-\infty}^{+\infty} z \mathbf{C F}(z, \lambda) \mathrm{d} z}{\int_{-\infty}^{+\infty} \mathbf{C F}(z, \lambda) \mathrm{d} z}
$$

Calculations of the contribution functions and of the effective heights of the line formation were included into the model calculations as a part of the DELO method. They are used for the interpretation of the results obtained from the numerical modeling. 


\subsection{Model calculations}

All the above steps of the numerical simulation of the magnetic structures are summarized in the scheme of Fig. 4.9, which presents the modeling consisting of the two big parts of the simulation process: the construction of the physical model of the facula (twocomponent, 3D simulation box), and radiative transfer calculations in the box for different inclinations $(\theta)$ of the line-of-sight to the vertical direction, simulating center-to-limb observations of photospheric small-scale magnetic structures.

The whole procedure begins with the choice of the spectral line and the assignment of free parameters and ends with the output:

$\diamond$ data cubes containing synthetic Stokes vector $\boldsymbol{I}\left(x^{\prime}, y^{\prime}, \lambda\right)$ for each $\mu=\cos \theta$, where $x^{\prime}, y^{\prime}$ are the spatial coordinates in the plane perpendicular to the LOS,

$\diamond$ corresponding contribution function to the spectral line depression.

The spatial size of simulated intensity maps is $\left(\mu \cdot 3^{\prime \prime}\right) \times 2^{\prime \prime}$ with a spatial sampling of $25 \mathrm{~km} \sim 0.035^{\prime \prime}$. The spectral sampling is $\Delta \lambda=10 \mathrm{m \AA}$. Due to the symmetry of the intensity profiles (macroscopic velocities were neglected) only one half of the spectral line from the continuum to the line center was calculated.

In order to simulate the limited spatial resolution of the observation caused by the diffraction limit of the telescopes and seeing conditions the synthetic 2D maps for each wavelength position were convolved with Gaussian functions to produce spatial resolution of $0.25^{\prime \prime}, 0.5^{\prime \prime}$ and $1.0^{\prime \prime}$ as in Fig. 4.10, which approximately correspond to the spatial resolutions of our data observed in the broadband channel of the FPI in the VTT (speckle reconstructed), in narrowband of the FPI (deconvolved with the instantaneous OTF) and to the moderate resolution of the 1D spectrograms observed with GCT, respectively.

For comparison with the FPI observations the calculated intensity profiles were additionally convolved in wavelength with the Airy function of the spectrometer (Sect. 2.2.1, Fig. 2.8).

Repeating the described simulations for each line and for different sets of free parameters a collection of synthetic data cubes $\boldsymbol{I}\left(x^{\prime}, y^{\prime}, \lambda, \mu\right)$ was generated, which now can be analyzed and compared with the observations. 
Free parameters of the model:

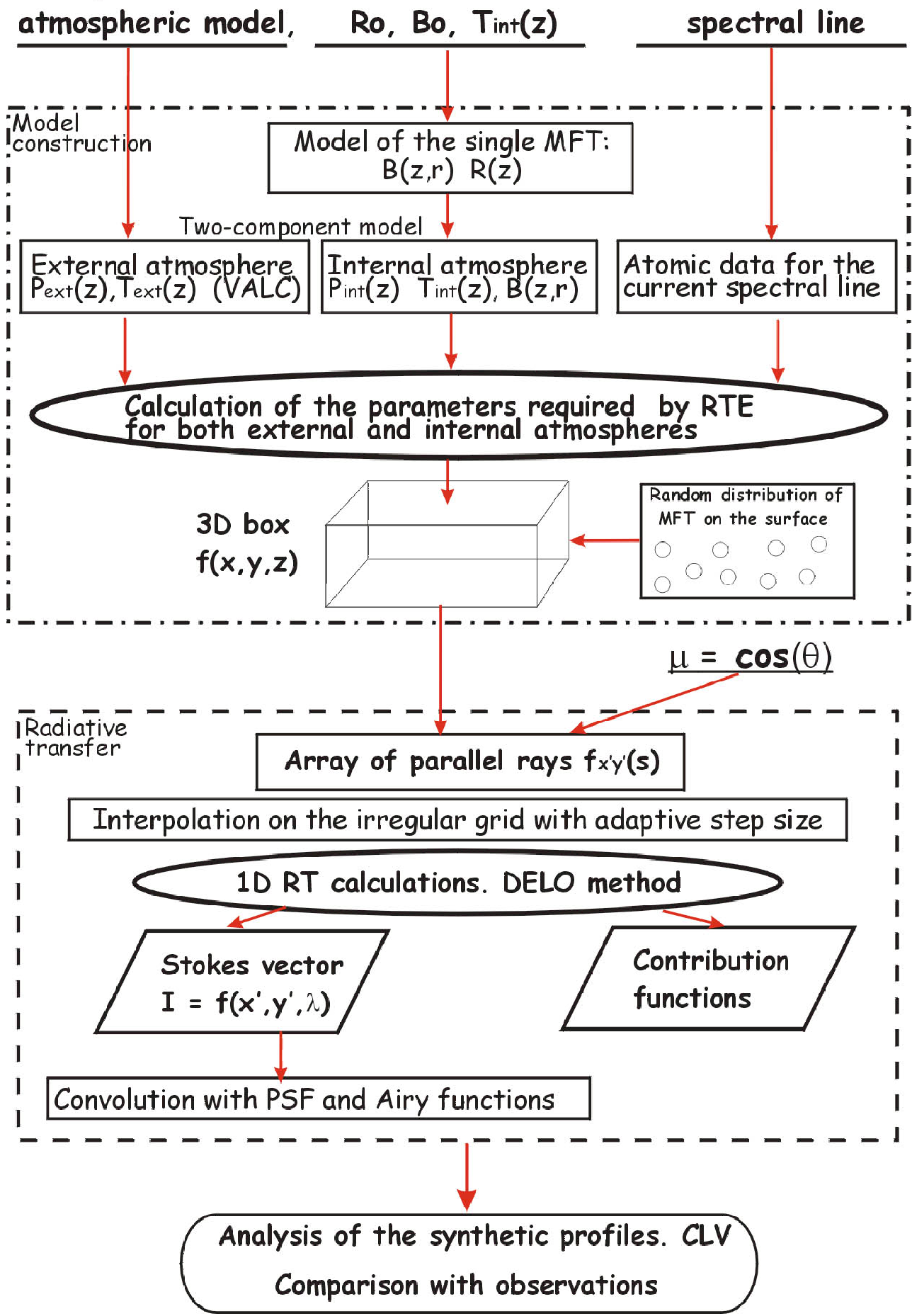

Figure 4.9: Scheme of the numerical modeling 


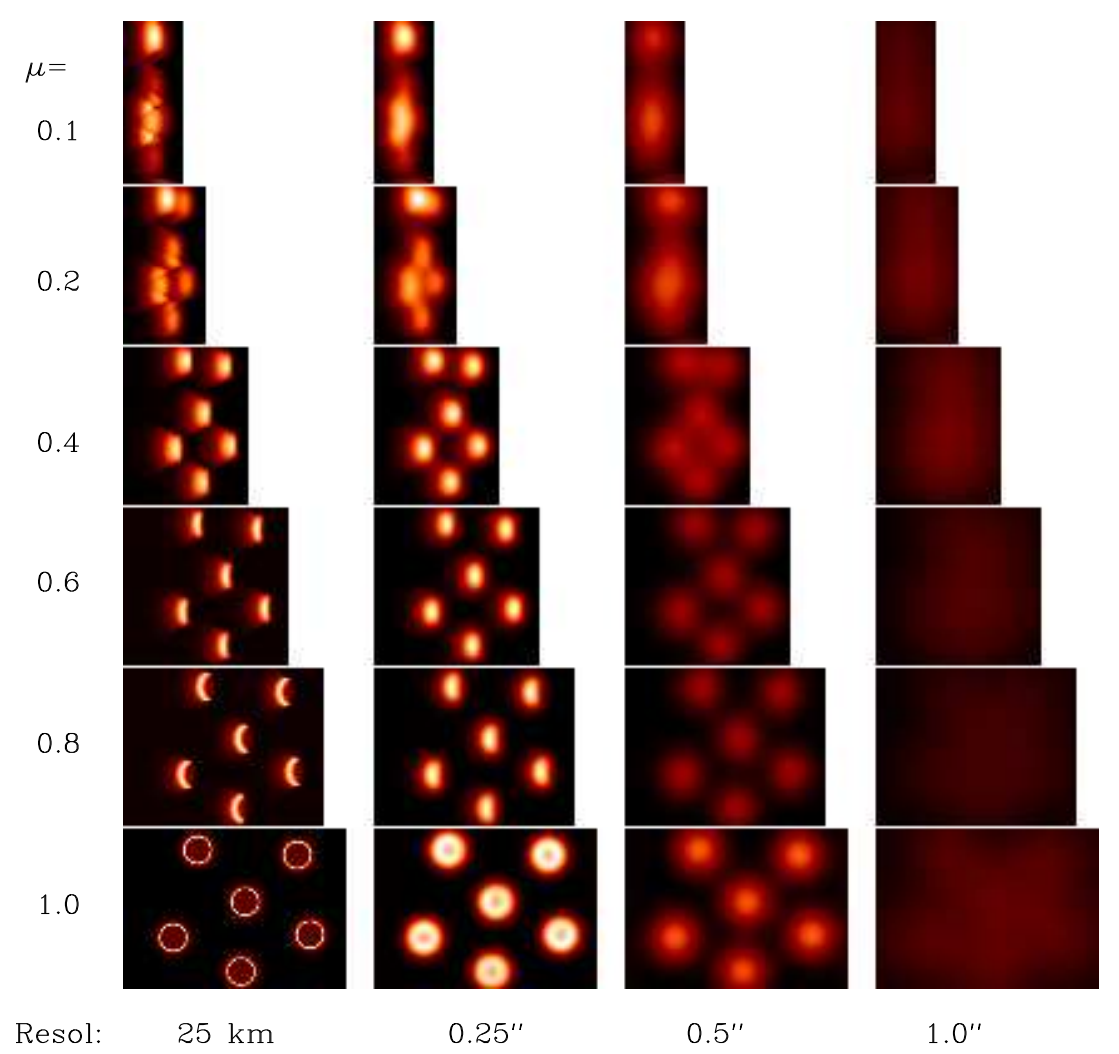

Figure 4.10: Example of the CLV of synthetic intensity maps for different spatial resolutions. 


\section{Results of numerical modeling and comparison with observations}

In this chapter we analyze the results of the performed numerical calculations. A large number of numerical models was generated for different sets of free parameters, and for each of them the radiative transfer problem was solved. Photometric and spectropolarimetric properties of the synthetic magnetic structures were compared with the typically observed characteristics of polar faculae on the Sun. Many sets of free parameters lead to numerical models, which are not able to satisfy the observational constraints. The other, successful models allow to conclude about the structure of PFs.

In the following sections we analyze the effects of the number of the MFTs along the LOS and of the thermal structure of flux tube interior on the properties of the radiation from the magnetic structures. We discuss brightness contrasts, sizes, fine structure of magnetic field and intensity distribution, properties of the Stokes $I$ and $V$ intensity profiles, and also center-to-limb variation of some of the parameters. Finally, we test the reliability of the standard calibration techniques which are used for the magnetic field measurements in polarimetric data observed at large heliocentric angles.

\subsection{Effect of many MFTs along the ray}

Magnetic flux tubes are tiny structures in the solar atmosphere. At the photospheric level of $z=0 \mathrm{~km}$ they have a diameter $d \sim 200 \mathrm{~km}$ or even smaller. And although they expand upwards, reaching in higher layers of the photosphere $d=1^{\prime \prime} \sim 700 \mathrm{~km}$, they still remain optically thin structures with a small contribution to the emergent intensities.

However, it was found from the observations that intensity profiles from PFs differ strongly in many characteristics from the averaged profiles from the surrounding quiet atmosphere. To produce such effects the MFTs, which constitute PFs, should have a large contribution along the integration path, which in turn can be ensured only by a sufficiently large number of small-scale MFTs traversed by the rays in the resolution element.

In order to study the effect of the number of MFTs along the integration path on the photometric and spectropolarimetric properties of the radiation from magnetic elements we have fixed all the free parameters of the model (line $\lambda 6302.5, \mu=0.2, B_{0}=1400 \mathrm{G}$, $R_{0}=100 \mathrm{~km}$, hot cloud model as the internal temperature), and have varied only the number of flux tubes in the simulation box. For the three earlier adopted random distributions of the MFTs on the surface (N1, N2, N3, see Fig. 4.3) numerical modeling and radiative transfer calculations were performed. The synthetic intensity maps were convolved with a smearing function simulating limited spatial resolution (seeing $\sim 0.3^{\prime \prime}$ ). 

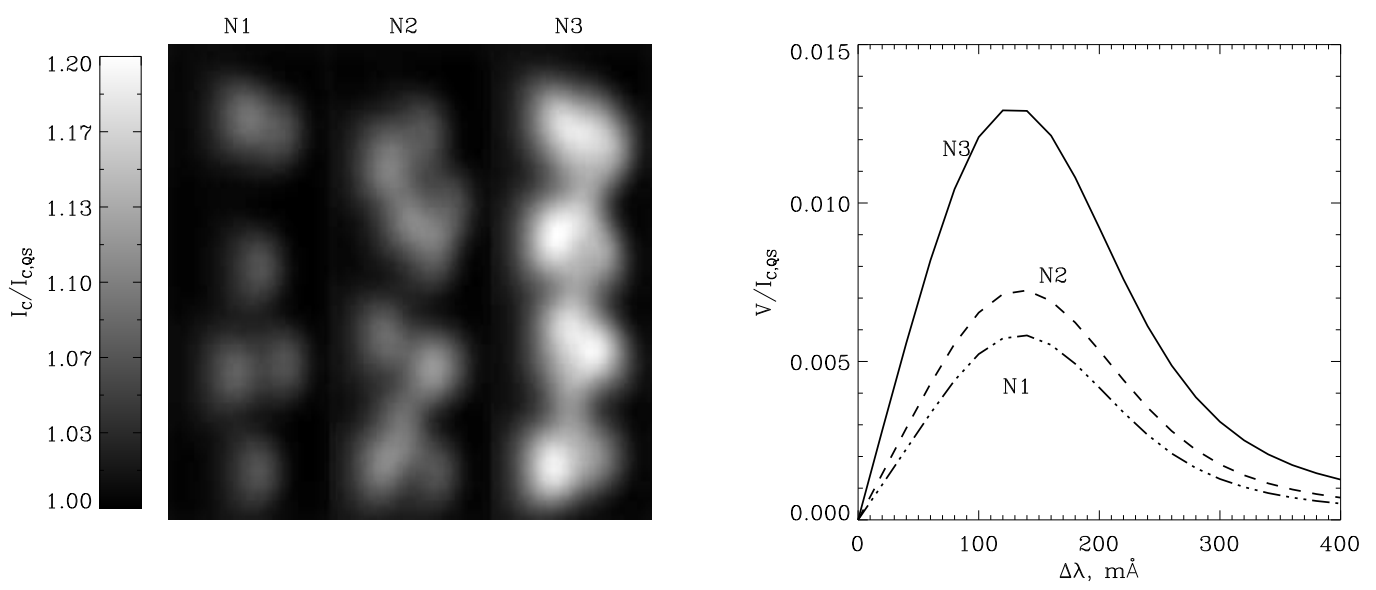

Figure 5.1: Left panel: The continuum intensity maps calculated at $\mu=0.2$ from the three realizations of the MFTs on the surface. The image sizes are $0.7^{\prime \prime} \times 2^{\prime \prime}$. Right panel: red lobes of the corresponding Stokes $V$ signals of the $\lambda 6302.5$ line.

It can easily be seen (Fig. 5.1) that the distributions N1 and N2 produce very low contrasts and weak $V$ amplitudes, and therefore are not appropriate for the modeling of PFs. The most populated distribution $\mathrm{N} 3\left(N_{\mathrm{MFT}}=21, f_{\text {mod }}=0.2\right.$, Sect. 4.1.5) produces more reasonable characteristics of the intensity profiles, though the amplitudes and contrasts are still slightly weaker than the strongest observed in PFs. To achieve a better correspondence of the synthetic faculae with the typically observed ones, another free parameter of the model $R_{0}$ can be used to tune the model and to control the contribution of the MFTs to the emergent intensity. After several numerical experiments it was found that by adopting the flux tube raduis $R_{0}$ in the range from $100 \mathrm{~km}$ to $150 \mathrm{~km}$ a good agreement with the observations can be achieved.

\subsection{Effect of internal thermal model}

Temperature is a determinative factor in the process of spectral line formation, which affects many observed properties of intensity profiles such as Doppler width, continuum and line core contrasts, line depression, and amplitudes of polarimetric signal. From the analysis of the observations (Sect. 3.3) it was found that the iron lines observed in PFs possess several peculiar features (Fig. 3.4 and Fig. 3.5), which strongly distinguish facular atmospheres from the surrounding quiet photosphere, and which are very likely due to the thermal structure of the faculae:

- strong contrast both in the continuum and line core for all three lines;

- in the Fe II 6149.3 line the Stokes $I$ profiles from PFs $\left(I_{\mathrm{PF}}\right)$ are substantially stronger and broader than the averaged intensity profile from the surrounding quiet Sun photosphere $I_{\mathrm{QS}}$;

- in the Fe I 6301.5 line $I_{\mathrm{PF}}$ show almost the same line depression as $I_{\mathrm{QS}}$ but appear to be slightly broader than $I_{\mathrm{QS}}$;

- in the Fe I 6302.5 line $I_{\mathrm{PF}}$ are slightly weaker and broader than $I_{\mathrm{QS}}$. 
These properties were found from the observations with both telescopes and in all data samples without any exception and so such behavior seems to be inherent to the facular atmospheres. Therefore, first of all we have tried to reproduce by numerical calculations these distinguishing features of PFs, using these properties as a criterion to choose the most plausible thermal model of the flux tube atmosphere for further model calculations. As it was described in Sect.4.1.3, three temperature stratifications of the flux tube interior were adopted as guess models for the numerical modeling:

- hot wall model (HW), with the internal temperature $T_{\text {int }}$ lower than the external $T_{\text {ext }}$ at the same geometrical level;

- hot cloud (HC), with $T_{\text {int }}(z<0)<T_{\text {ext }}$ and $T_{\text {int }}(z>0)>T_{\text {ext }}$;

- equal temperatures (EQ) at the same geometrical height $T_{i n t}=T_{\text {ext }}=T_{\mathrm{VALC}}$.

For each of the above temperature models a corresponding flux tube atmosphere was generated taking into account a partial evacuation of the tube in accordance with the pressure ballance (Sect. 4.1.2). Parameters of the model $B_{0}=1400 \mathrm{G}$ and $R_{\mathrm{MFT}}=\infty$ were adopted for the current calculations. The latter means that for the sake of simplicity and in order to exclude many other effects, for the purpose of demonstration and explanation, the radiative transfer equation was solved under the assumption of a plane parallel atmosphere at $\mu=\cos \theta=0.3$ and for the ray along LOS always staying in the flux tube atmosphere. For the thus created three flux tube atmospheres and for the quiet Sun (VALC) synthetic Stokes $I$ and $V$ profiles were calculated. In order to find out how the partial evacuation of the MFT and different temperature stratifications change the heights of the spectral line origin the corresponding contribution functions (CF) to the line depression were calculated. In order to distinguish between broadening of the spectral line due to thermal effects (Doppler broadening) and due to the unresolved Zeeman splitting we have additionally synthesized Stokes $I$ profiles emerging from the flux tube interior but assuming zero magnetic field strength.

The results of the calculations for Fe II 6149.3 and for the pair of lines Fe I 6301.5 and 6302.5 are presented in Fig. 5.2 and 5.3, respectively. Similarly to the representation of the observed profiles (Fig. 3.4 and Fig. 3.5) the synthetic profiles are also presented in two normalizations: to the continuum of the surrounding quiet Sun $I / I_{\mathrm{C}, \mathrm{QS}}$ (to compare contrasts), and to the local continuum $I / I_{\mathrm{C}}$ (to compare line depressions).

At large heliocentric angle the geometrical path of the ray through the atmosphere is longer than at the center of the disk, accordingly the spectral lines originate from higher layers, and contribution functions for the VALC atmosphere at $\mu=0.3$ (Fig. 5.2, 5.3 upper panel) are shifted upwards by approximately $100 \mathrm{~km}$ compared to the calculations at $\mu=1.0$ (Fig. 4.8). However, due to the reduced pressure inside the MFT the opacity both in continuum and line core drops, and accordingly the region of the line formation is shifted to lower layers of the photosphere. As a result, the corresponding CFs for the flux tube atmospheres and EQ temperature stratifications (Fig. 5.2, 5.3) are quite similar to the CFs from the quiet Sun but at $\mu=1.0$ (Fig. 4.8).

The effect of different temperature stratifications on the heights of origin of a spectral line is more complicated. It is demonstrated in the Figs. 5.2 and 5.3 (two lower rows) corresponding to the model calculations for $\mathrm{HC}$ and $\mathrm{HW}$ temperature models, respectively. The results can be understood taking into account the physical mechanisms, which define the formation heights. 

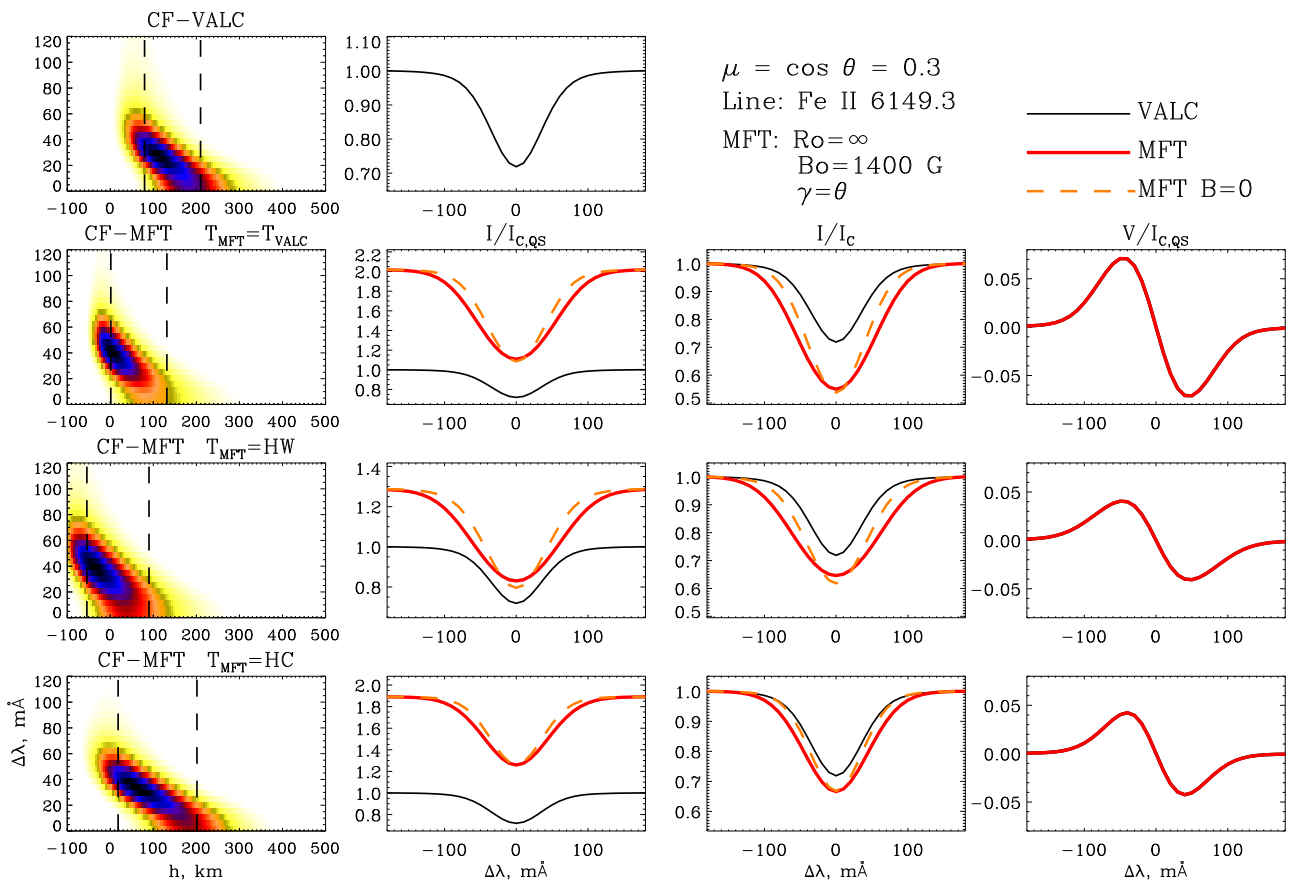

Figure 5.2: Synthetic Stokes $I$ and $V$ intensity profiles (Fe II 6149.3) with corresponding contribution functions (CF) for the magnetic flux tube (MFT) atmospheres (red solid lines) with three temperature stratifications compared with the results of the calculations in external photosphere (VALC, black solid lines). Dashed profiles are the Stokes $I$ profiles calculated for MFT atmosphere but without Zeeman broadening $(B=0)$. Dashed vertical lines in the plots with CFs point to the heights where line core and continuum are formed.

The increase of the temperature, for example, has the following consequences:

- continuum radiation originates from higher layers. Mechanism: additional ionization, excess of free electrons leads to the increase of the number of $\mathrm{H}^{-}$, which in turn makes the gas less transparent in continuum;

- line core of neutral iron lines is formed deeper: stronger ionization, less absorbers, low opacity;

- line core of the ionized iron line is formed higher: stronger ionization, more absorbers, higher opacity.

For the Fe II 6149.3 line it was found that for both models (HW and HC) the corresponding Stokes $I$ profiles from the MFT atmospheres are more depressed and broader than $I_{\mathrm{QS}}$ at the same $\mu$ (Fig. 5.2). But from the comparison of the synthetic $I$ profiles with the observation in the same line (Fig. 3.5) we can presume that for the line of ionized iron the HW model is more preferable than $\mathrm{HC}$. The hot cloud temperature stratification is less adequate because it produces intensity profiles with characteristics different from the observed: too strong line core contrast and relatively weak broadening. The latter is particularly surprising, because for the hot photosphere (HC) we would have expected broader profiles than from the cool HW model. However, this can be understood by the comparison of the corresponding CFs. For the HW temperature stratification the line originates from deeper layers where temperatures are larger than in the $\mathrm{HC}$ case at the 

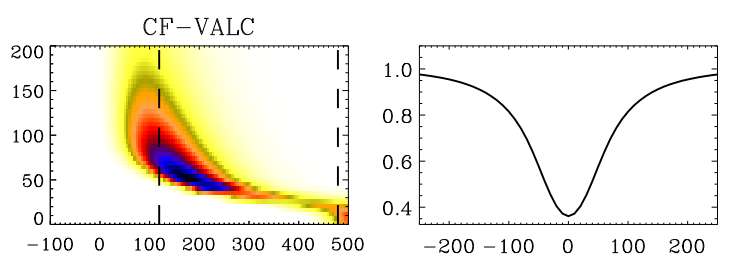

$\mu=\cos \theta=0.3$
Line: Fe I 6301.5

Line: Fe I $6301.5 \quad$ VALC

MFT: $R O=\infty$ $\mathrm{Bo}=1400 \mathrm{G} \quad \mathrm{MFT}$
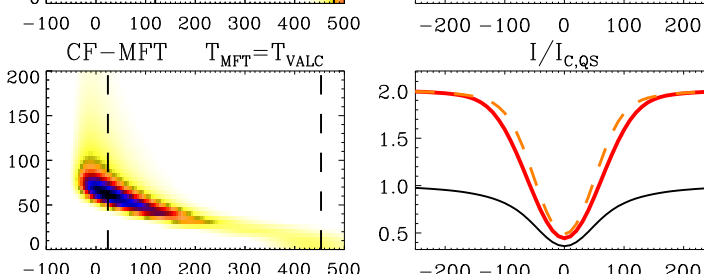
$\gamma=\theta$

$--\quad-\mathrm{MFT} B=0$
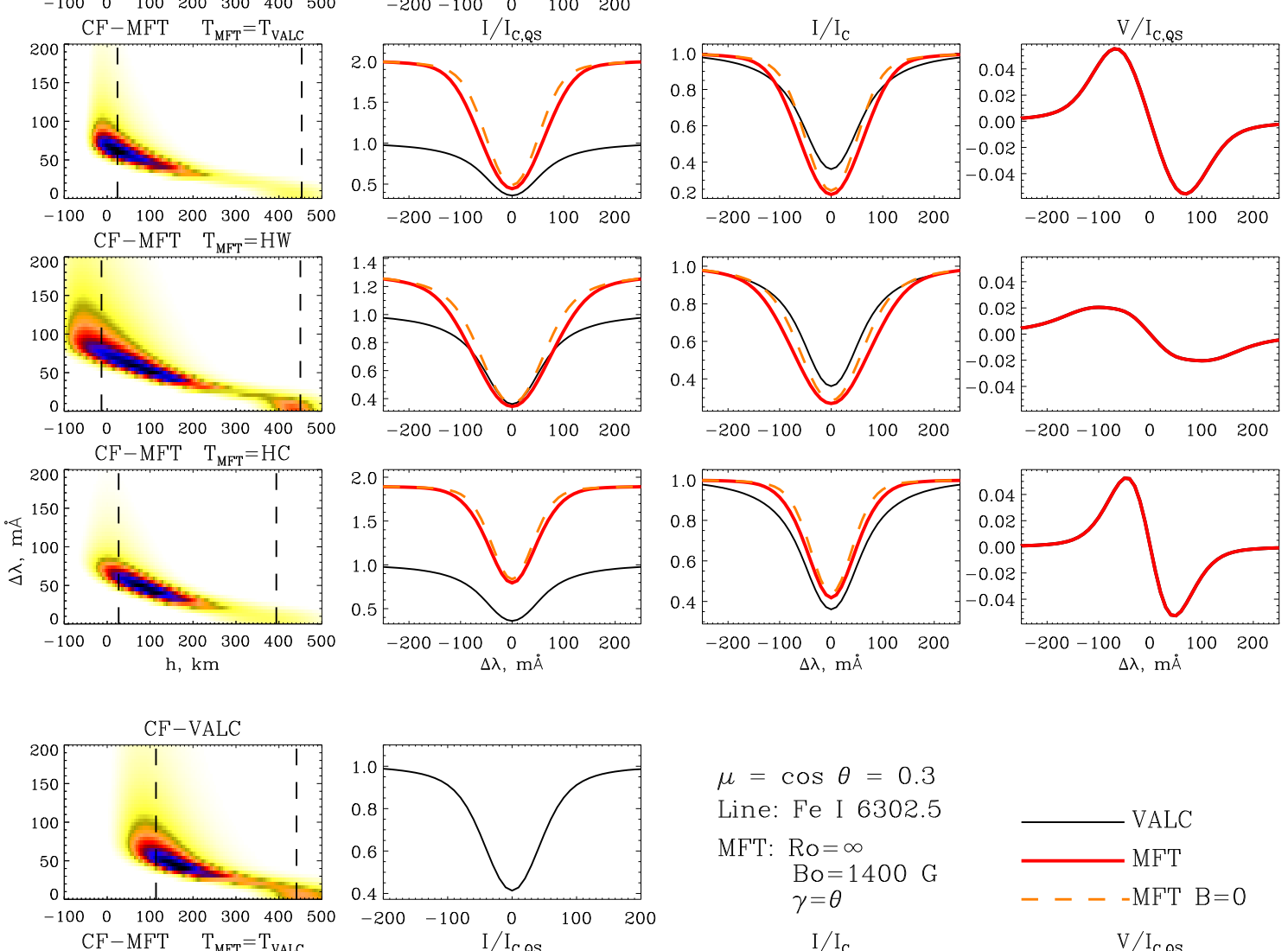

$\mu=\cos \theta=0.3$

Line: Fe I 6302.5

MFT: $R O=\infty$

$\mathrm{Bo}=1400 \mathrm{G}$
$\gamma=\theta$

VALC

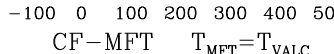
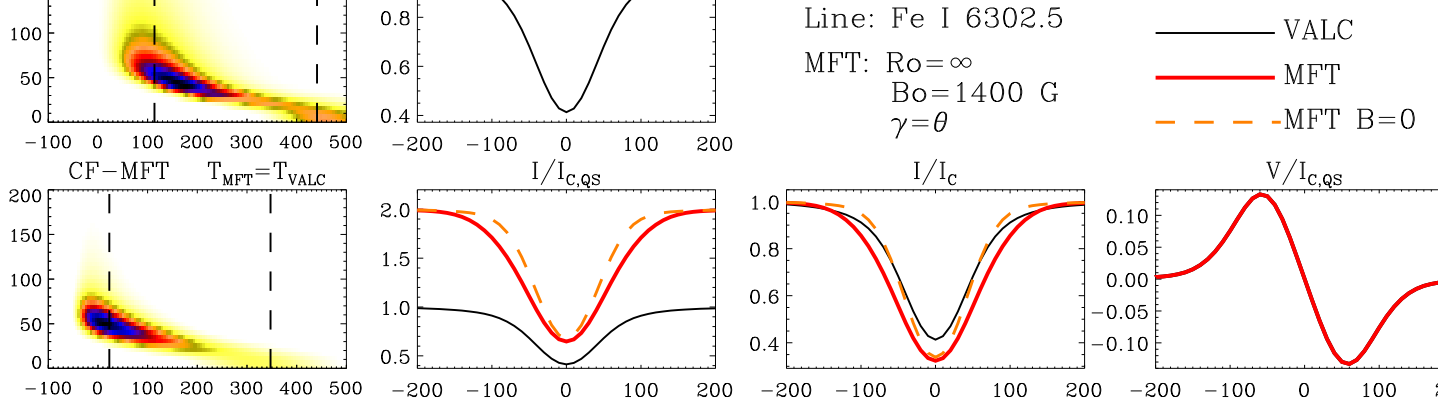

$\begin{array}{ccccccc}-100 & 0 & 100 & 200 & 300 & 400 & 50 \\ \mathrm{CF}-\mathrm{MFT} & \mathrm{T}_{\mathrm{MFT}}=\mathrm{HW}\end{array}$
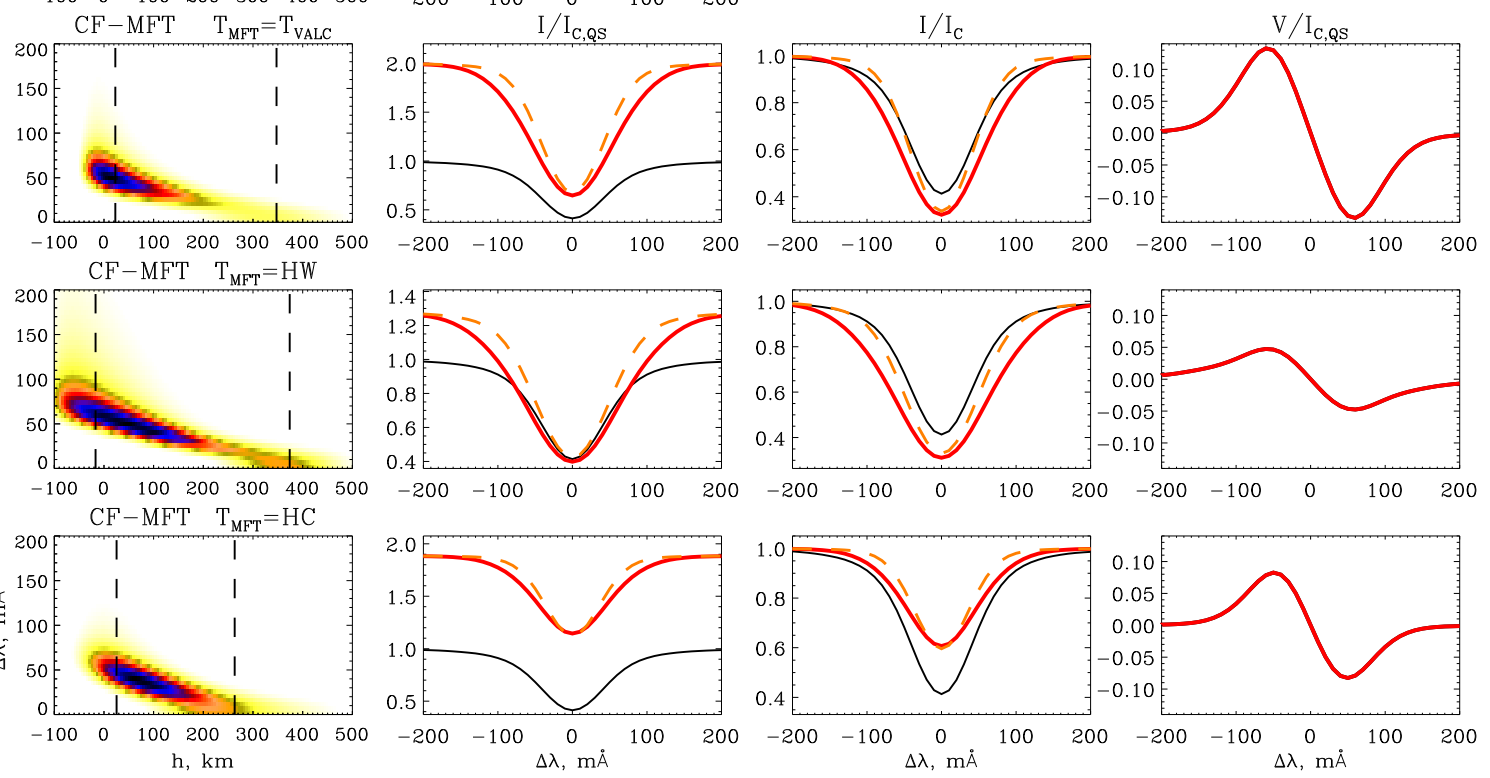

Figure 5.3: The same as Fig. 5.2 but for the spectral lines of neutral iron Fe I 6301.5 and 6302.5 . 
same optical depth, which results in broad intensity profiles even with neglected Zeeman splitting (Fig. 5.2 dashed profiles).

As to the Fe I 6301.5 and 6302.5 spectral lines (Fig. 5.3), the conclusion from the comparison of the properties of the synthetic and observed profiles is opposite! For the line of neutral iron the HC model is more preferable. The hot wall temperature stratification for both lines results in a very low line core contrast and the intensity profiles are more depressed than $I_{\mathrm{QS}}$, which contradicts the observational constraints: strong line core contrasts and weaker/equal line depression.

An interesting difference between the HW and HC models in the case of neutral iron lines is that the hot wall model enlarges the range of heights where the lines are formed (CFs are stretched, temperature difference between the layers of the continuum and line core formation is big), resulting in increased line strengths. The HC model shows an opposite behavior, it squeezes the CFs, resulting in the weakening of the lines. It is also interesting to note that for the HW model the amplitudes of the corresponding Stokes $V$ profiles are weaker than for the HC model, in spite of the opposite situation with the depression: strong depression in case of $\mathrm{HW}$ and weak in case of HC. The explanation is in the stronger Doppler broadening of the absorption profiles formed in the HW photosphere compared to the HC (the mechanism is the same as for the ionized line: deeper and hotter layers of formation). It results in a weak Stokes $V$ profile, which is the difference between the left circularly and right circularly polarized and Doppler broadened profiles of the Zeeman components.

The main conclusion from the presented calculations is that it is impossible to reproduce all observed properties of Stokes $I$ profiles for the three spectral lines under consideration using only one of the three initially adopted temperature models. Depression and contrasts of the ionized iron line are explained better by the HW model, while for the spectral lines of neutral iron the $\mathrm{HC}$ model is more preferable.

A solution of this contradiction is to create another thermal model, able to satisfy the observational constraints for all lines. Fortunately, such a model is not difficult to deduce, because the lines originate from different regions, and the sought temperature stratification should be simply a combination of both HW and HC: the interior of the MFT should be cooler than the external plasma at the same geometrical level in the low photosphere where the $\lambda 6149.3$ line is formed and, accordingly, hotter in the upper layers where line cores of the $\lambda 6301.5$ and $\lambda 6302.5$ lines are formed. Such a temperature model (hereafter referred to as $\mathrm{HC} 1$ ) was constructed by modifying the initial HC model. The crossing point, where $T_{\text {int }}=T_{\mathrm{VALC}}$, was shifted from $z=0 \mathrm{~km}$ in the initial HC model up to the height $z=120 \mathrm{~km}$. The thus derived internal temperature stratification is presented in Fig. 5.4 (black thin line). It was compared with the internal temperature obtained by Bellot Rubio et al. (2000) by means of inversion of Stokes profiles emerging from plage regions (Fig. 5.4 red dashed line). They agree well in the range of heights from $-100 \mathrm{~km}$ to $300 \mathrm{~km}$. According to Bellot Rubio et al. for $z>300 \mathrm{~km}$ the performed inversion gives uncertain results.

The model calculations were repeated as described above for the new thermal model of the flux tube interior (HC1), and it was found that the synthetic Stokes $I$ and $V$ intensity profiles are similar to the typically observed Stokes parameters for all three spectral lines (Fig. 5.5), and in particular the distinguishing features of PFs mentioned in the beginning of this section are well reproduced by the adopted model. 


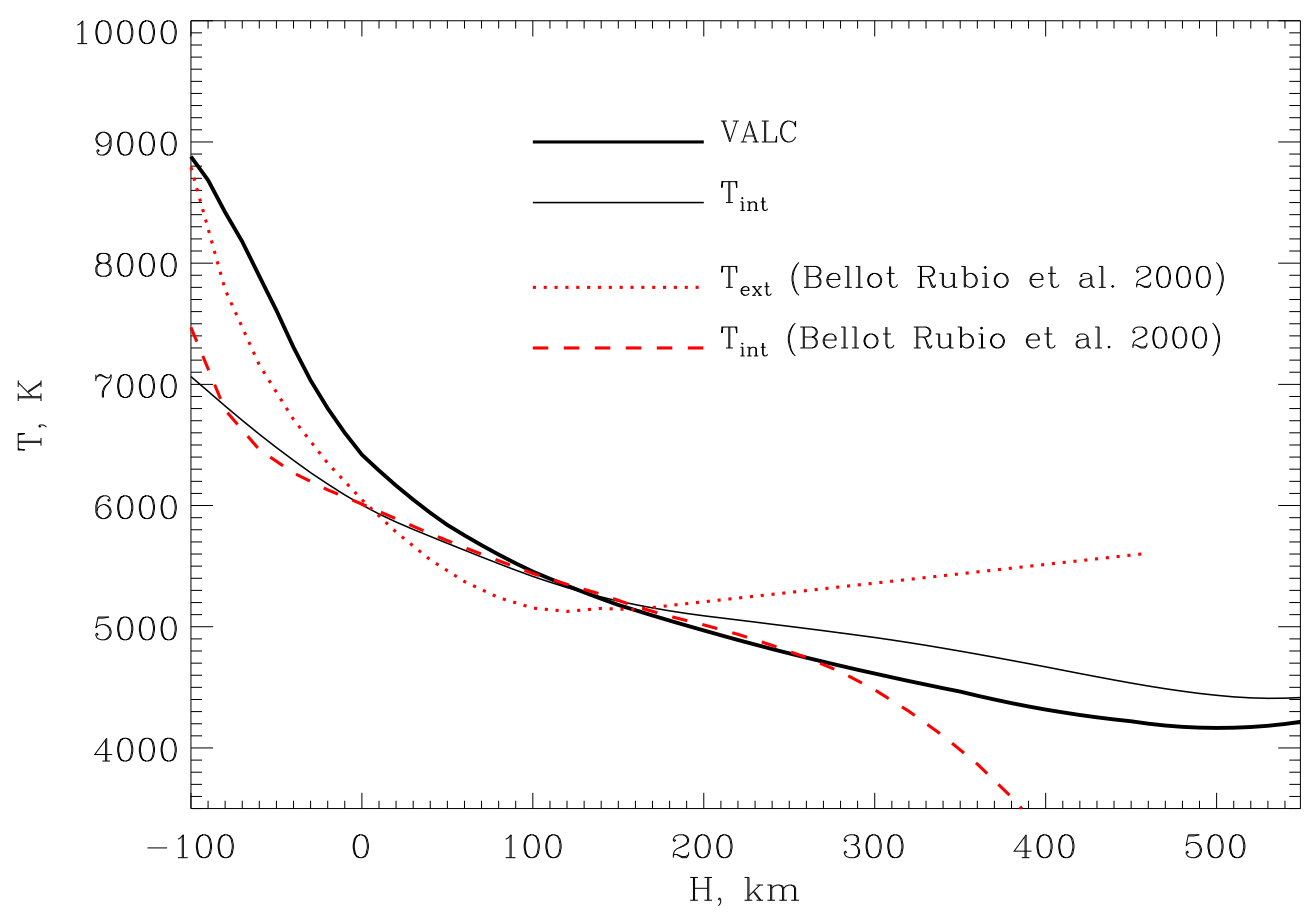

Figure 5.4: A modified internal temperature model HC1 (black thick line) and the VALC temperature (black thin line) compared with internal (red dashed line) and external (red dotted line) obtained from inversion of Stokes profiles by Bellot Rubio et al. (2000).
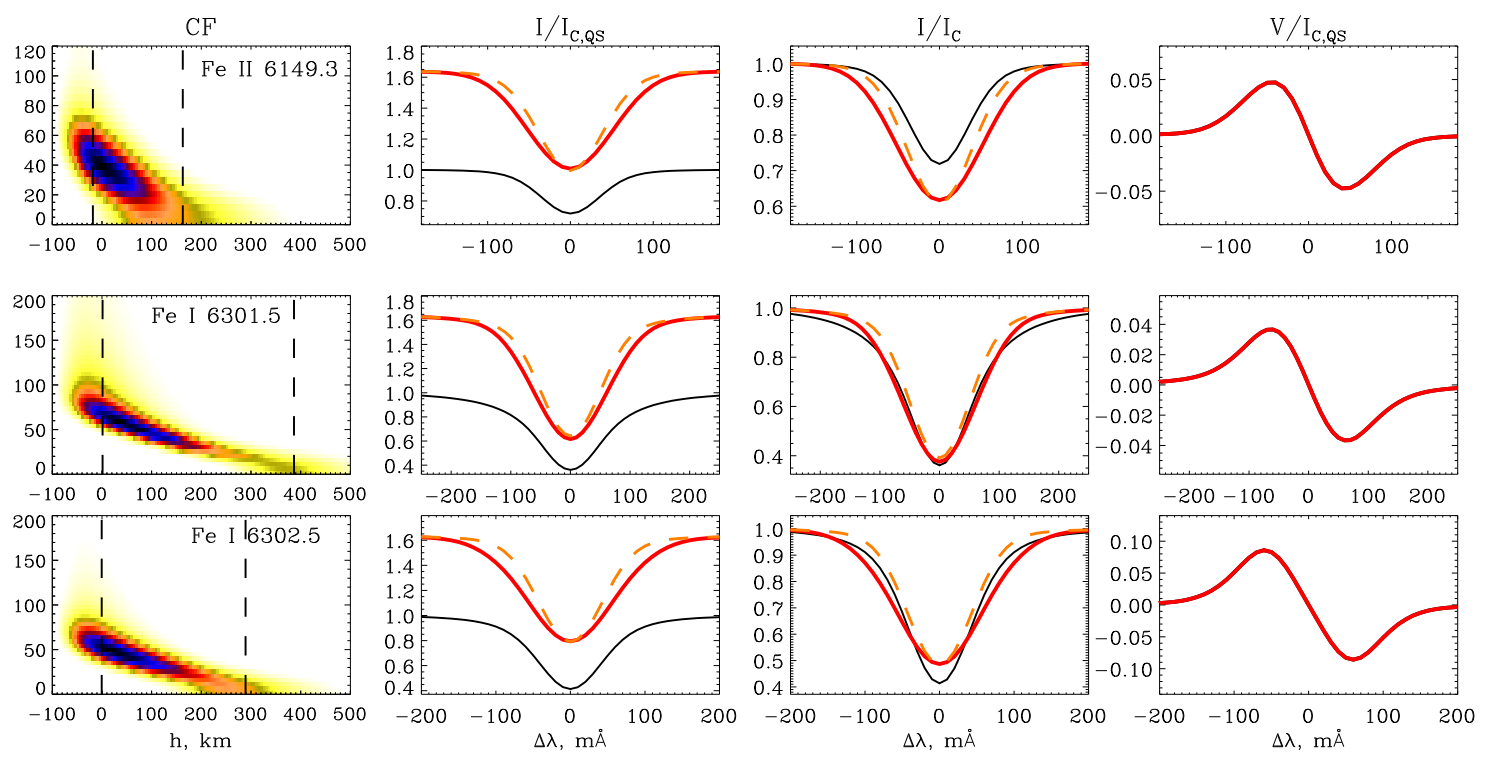

Figure 5.5: Synthetic Stokes $I$ and $V$ profiles $(\lambda 6149.3, \lambda 6301.5, \lambda 6302.5)$ with the corresponding $\mathrm{CFs}$ for the modified temperature model $\mathrm{HC} 1$. The line types have the same meaning as in Fig. 5.2. 
In such a way, from the above analysis we conclude that the $\mathrm{HC} 1$ thermal model with $T_{\text {int }}(z<120)<T_{\text {VALC }}$ and $T_{\text {int }}(z>120)>T_{\text {VALC }}$ is an appropriate choice of a temperature stratification inside MFTs to be used in the simulations. We note the importance of the use of several spectral lines with different regions of formation, which allows studying the stratification of the parameters in the solar photosphere in a wide range of heights.

\subsection{Spectropolarimetric profiles from synthetic faculae}

The analysis presented in the previous sections was made under the assumption of planeparallel atmosphere, i.e. we have assumed that rays along the LOS always stay in the internal atmosphere of the magnetic structure. Effects of the magnetic intermittency in the directions parallel and perpendicular to the LOS were neglected for the sake of clearness and simplicity. Under these conditions the internal temperature model HC1 (Fig. 5.4) was deduced by comparison of the spectral properties of the synthetic profiles with the observed ones in three spectral lines.

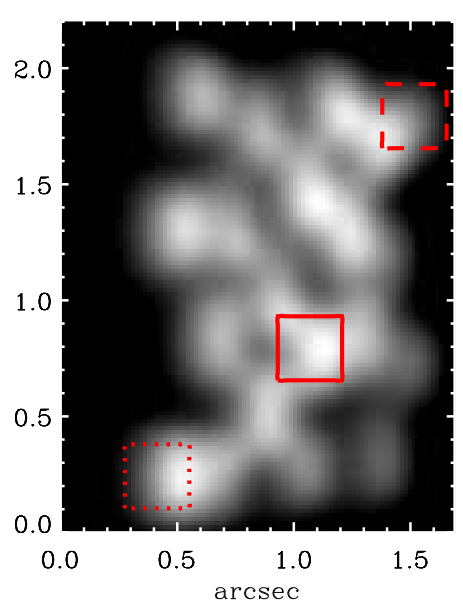

Figure 5.6: Synthetic continuum intensity map at $\mu=0.4$. Solid, dashed and dotted squares show the regions over which the intensity profiles of Fig. 5.7 were averaged. Limb side is left.

In this section we present results of more realistic simulations. The synthetic Stokes $I$ and $V$ profiles are calculated from a model without the above simplifications, i.e. multi-ray $1.5 \mathrm{D}$ radiative transfer calculations in $3 \mathrm{D}$ box were performed. For the presented simulations we have adopted: $\mu=0.4$, internal $T$ model $\mathrm{HC} 1$, $B_{0}=1400 \mathrm{G}, R_{0}=150 \mathrm{~km}$ and distribution N3. As usually, the spatial resolution of $0.5^{\prime \prime}$ and spectral resolution of $\sim 40 \mathrm{~mA}$ were simulated in accordance with the parameters of observations in the narrow band of the Fabry Perot spectrometer in the VTT.

The spatial size of the synthetic data cubes $I\left(x^{\prime}, y^{\prime}, \lambda\right)$ and $V\left(x^{\prime}, y^{\prime}, \lambda\right)$ is $2.2^{\prime \prime} \times 1.7^{\prime \prime}$. Obviously, the properties of intensity profiles depend on the spatial position $\left\{x^{\prime}, y^{\prime}\right\}$ in the simulated intensity pattern, because each individual ray has different contributions to the emergent intensity from the MFTs along the integration path. For demonstration purposes three regions in the intensity map were selected (Fig. 5.6), over which the Stokes $I$ and $V$ profiles were averaged:

1) the region containing the brightest point in the middle of the cluster with a large contribution of MFTs along the line-of-sight, both line core and continuum intensities are formed within flux tubes (solid square in Fig. 5.6);

2) the region at the center side of the cluster, the line core is formed in the non-magnetic plasma, the continuum in a magnetically intermittent atmosphere (dashed square);

3) the region at the limb side of the cluster, the line core is formed in an intermittent photosphere, the continuum in the non-magnetic plasma (dotted square). 
The Stokes $I$ and $V$ profiles synthesized in three spectral lines were averaged in each of the selected regions. Their properties (continuum and line core contrasts, depressions of $I$ and amplitudes of $V$ ) were compared with each other and with profiles from the surrounding quiet Sun as shown in Fig. 5.7.
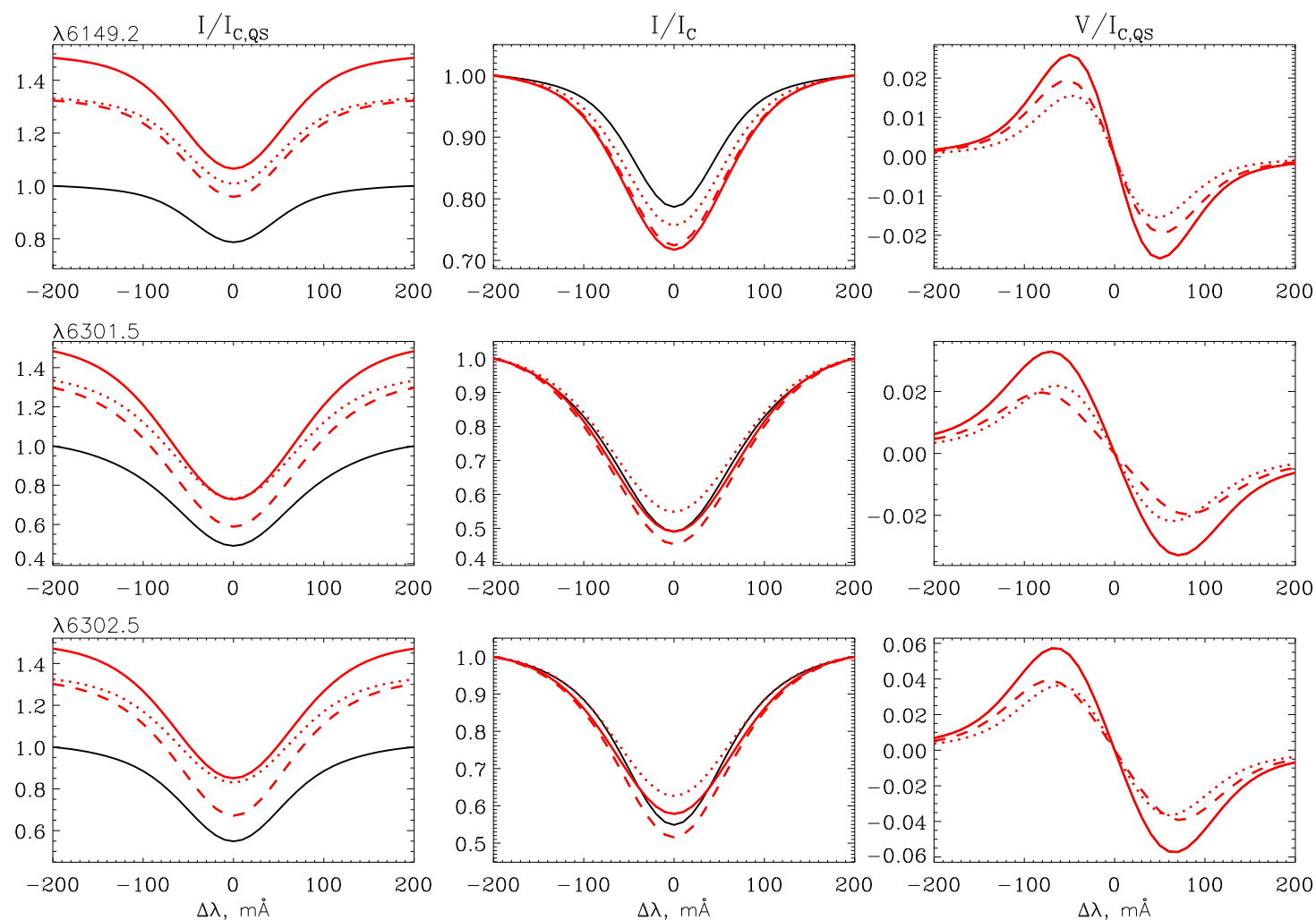

Figure 5.7: Spectropolarimetric profiles from synthetic facula at $\mu=0.4$. Black lines are profiles from the ambient quiet Sun, red lines from facula. Solid, dashed and dotted profiles emerge from the regions (1), (2) and (3) presented in Fig. 5.6 as squares with corresponding line types.

Middle of facula. Region 1. The intensity profiles (solid red lines in Fig. 5.7) have the highest contrasts in continuum and in line core, and also possess relatively strong $V$ amplitudes. The depressions of the $I$ profiles from the brightest point in the synthetic facula are close to those calculated under the assumption of a plane-parallel atmosphere with a ray staying always in a flux tube atmosphere (Sect. 5.2). All these effects are due to the large contribution of MFTs along the integration path to the intensities emerging from the middle of the apparent intensity pattern.

Profiles from the center side and the limb side are formed in the magnetically intermittent atmospheres, and therefore they exhibit a different behavior.

Center side. Region 2. The profiles (dashed lines) possess smaller contrasts at line cores compared to the region 1 , because the core intensities at the limb side are formed under conditions close to the external VALC photosphere. In the case of $\lambda 6149.3$ the line core contrast is still strong due to the low levels of formation which are more effected by the flux tube component than the upper layers where the cores of neutral iron lines are formed. The continuum contrast is weaker than from region 1 , due to the decreased contribution 
from MFTs along the rays in the resolution element compared to region 1 . The Stokes $V$ profiles are weaker than from region 1 for the same reason. The depressions of the neutral iron lines are larger. This is due to the increased difference of heights (accordingly higher temperature difference) between the regions of continuum and line core formation.

Limb side. Region 3. The line core contrasts from the limb side (dotted lines) are similar to those from region 1 . In both cases the line core radiation originates from approximately the same heights in the flux tube interior, while the continuum intensities are formed under different conditions. The continuum contrasts and $V$ amplitudes are weaker due to the small contribution of MFTs along the line-of-sight. The line depressions are weaker, because line core and continuum are formed in a narrower range of heights with smaller temperature difference.

In this way it was found that spectral properties of the radiation from faculae structures observed close to the limb are very much influenced by the magnetic intermittency of the atmosphere along the LOS. For the adopted model at $\mu=0.4$ with $R_{0}=150 \mathrm{~km}$ and rich populated with MFTs (distribution N3) the contribution from individual flux tubes is dominant in the middle of the simulated intensity pattern, however at the limb and central sides the inhomogeneity of the atmosphere results in a strong variation of the properties of the $I$ and $V$ profiles.

The simulated profiles agree well with the observation (cf. Sect. 3.3). Contrasts, depressions, intensity profile variations are well reproduced.

\subsection{Spatial distribution and fine structure}

In Sect. 3.6 the observational characteristics inherent to polar faculae were summarized. In this section we compare them with the results of the model calculations and test the ability of the numerical model to simulate some of the apparent properties of PFs, such as fine structure, sizes and spatial distribution of the brightness and polarimetric signal.

For the comparison with the observations we have chosen a model with the following parameters: $B_{0}=1400 \mathrm{G}, R_{0}=100 \mathrm{~km}$, MFT distribution: N3, $T_{\text {int }}=\mathrm{HC} 1$, $\mu=(0.4,0.2,0.1)$. A spatial resolution of $0.3^{\prime \prime}$ was simulated. The apparent spatial distribution of the apparent LOS magnetic field was obtained by estimating the amplitudes of the Stokes $V$ profiles in the $\lambda 6302.5$ line. The intensity maps calculated in the continuum close to the spectral lines (Fig. 5.8) are very similar to the ones observed with high spatial resolution (Fig. 3.2).

It was found that several apparent properties of the simulated structures are in a good agreement with the observations:

- high intensity contrasts, however decreasing towards the extreme limb;

- at $\mu=0.4$ the synthetic polar faculae appear as a cluster with a complex fine structure (subarcsec sizes) both in brightness and magnetic field strengths;

- at $\mu=0.2-0.1$ they consist of small bright structures organized in chains stretched parallel to the limb, the distance between the pearls in the chain is about $0.5^{\prime \prime}$;

- the distribution of the polarimetric signal is shifted towards disk center with respect to the continuum brightness. 

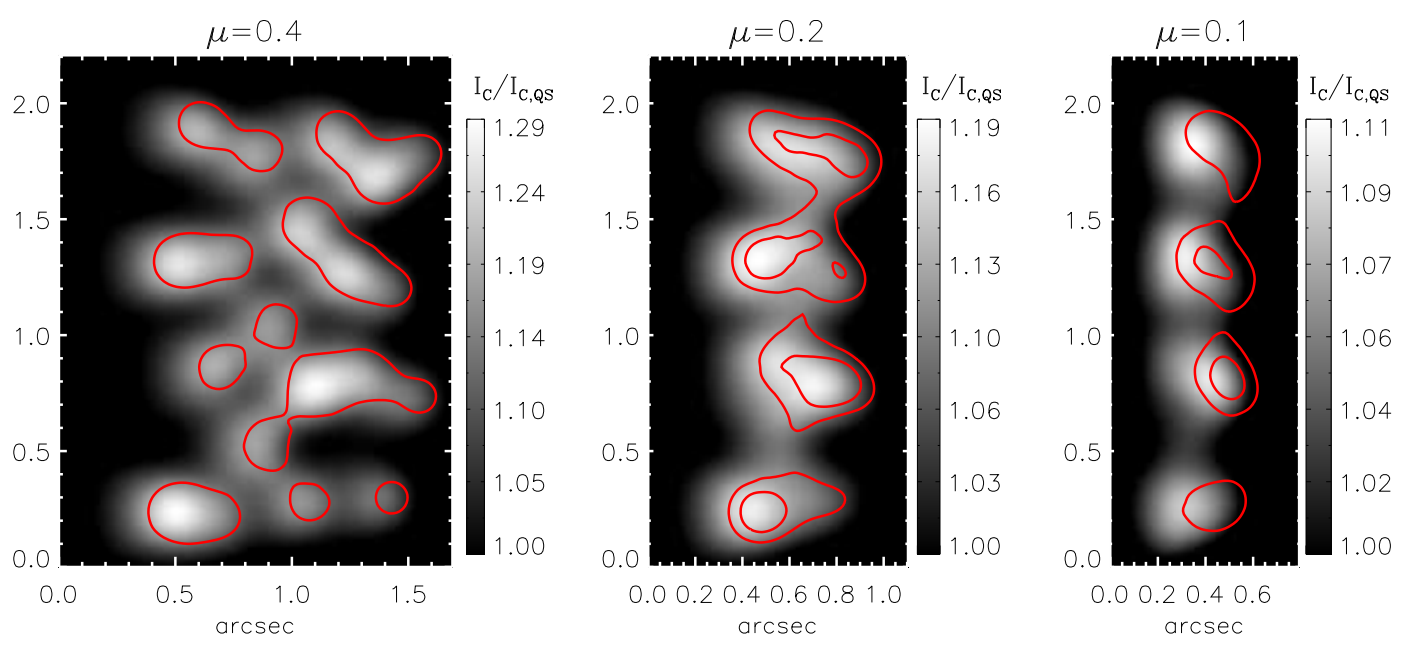

Figure 5.8: Synthetic continuum intensity maps normalized to the intensity of the surrounding quiet Sun and overplotted with the contours of the LOS magnetograms. Left side of the maps corresponds to the direction to the limb.

The similarities with the observed PFs and their fine structure and sizes are based on the adopted concept of small-scale MFTs. But the ability of the model to reproduce the observed shifts of polarimetric signal with respect to continuum intesity (Sect. 3.4) is remarkable. It demonstrates the reliability of the constructed model. The interpretation of the observed shifts without numerical simulation would not be straightforward because, as it was shown in the previous section, the amplitudes of the $V$ signal depend not only on field strengths and inclinations but also on the temperature along the LOS in a non-trivial way. Temperature affects opacities (height of formation), line strength and Doppler width of the lines, which in turn affect the Stokes $V$ signal. The enhancement of the brightness can be due to the reduced opacity along the LOS, which allows to see deeper and hotter layers of the photosphere. The numerical model considers all the effects in a consistent way and successfully simulates the observed properties of PFs.

\subsection{Center-to-limb variation}

The center-to-limb variation (CLV) of the observed intensity contrast and $V$ amplitudes of PFs were discussed in the Sect. 3.1.1 and Sect. 3.3.3. Polar faculae possess enhanced brightness near the limb, however the contrast monotonically decreases towards the extreme limb. No maximum of the apparent contrast in the observed range of heliocentric angles $(\mu=0.4-0.1)$ was measured. A similar behavior was found in the CLV of the Stokes $V$ amplitudes.

The radiative transfer calculations for each simulation box were performed for several inclinations of the LOS to the vertical axis of the model. We have used HC1 (Sect. 5.2, Fig. 5.4) as the internal temperature. Calculations were performed for $R_{0}$ equal to $100 \mathrm{~km}$ and $150 \mathrm{~km}$, and for MFTs distributions N2 and N3 (Sect. 4.1.5). The emergent Stokes profiles were convolved with Gaussians and with Airy function of the FPI spectrometer in 

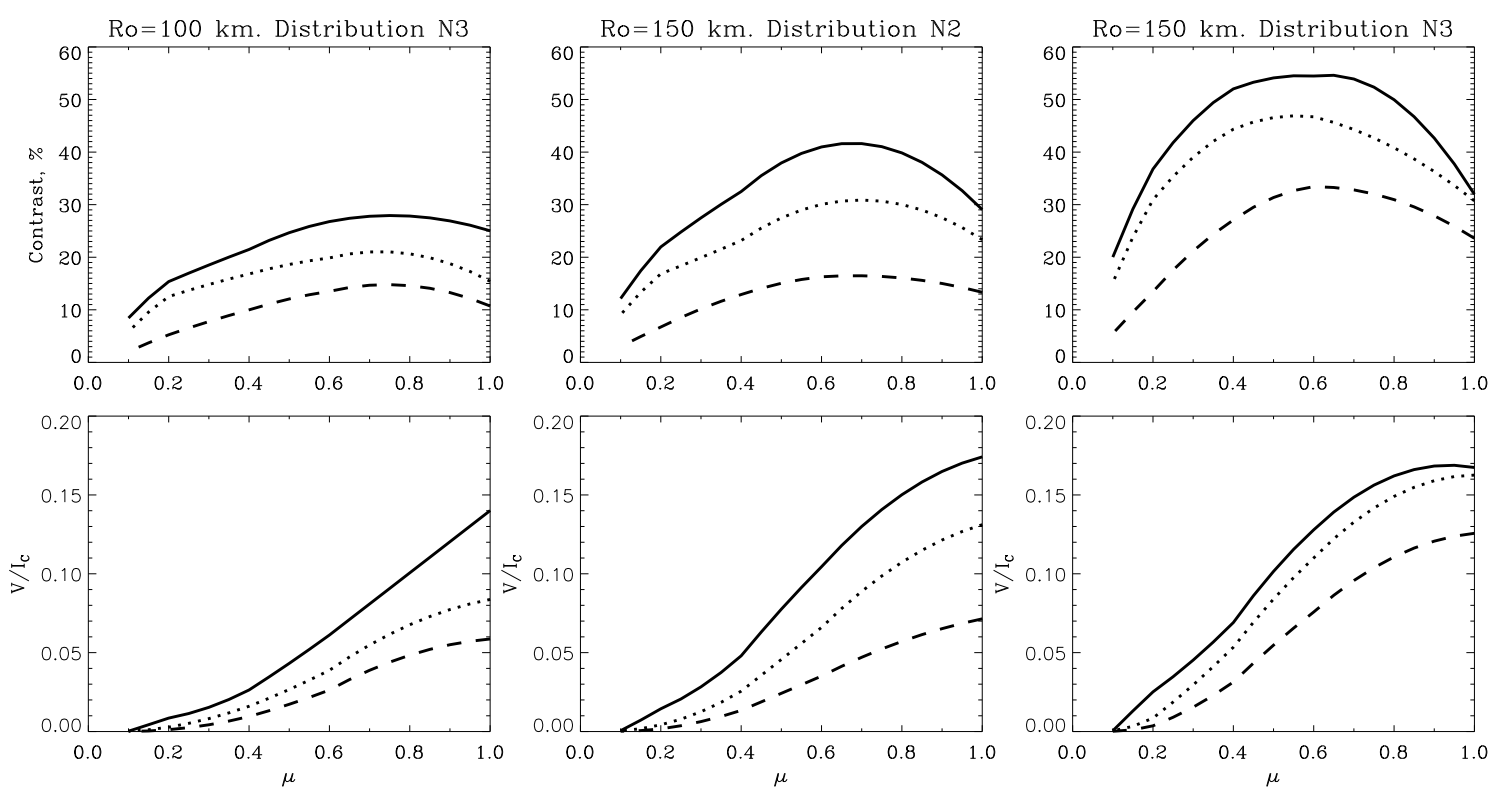

Figure 5.9: CLV of the continuum intensity contrast and the Stokes $V$ amplitude in the $\lambda 6302.5$ line for three different models. Solid, dotted and dashed lines denote the spatial resolution of $0.25,0^{\prime \prime} .5$ and $1^{\prime \prime}$, respectively.

order to simulate the limited spatial and spectral resolution of the observations as shown in Fig. 4.10. The brightness contrast is defined as $\left(I_{\max }(\mu) / I_{\mathrm{VALC}}(\mu)-1\right) \cdot 100$ and Stokes $V$ amplitudes were derived from the polarimetric signal in the $\lambda 6302.5$ line.

The resulting center-to-limb variations are presented in Fig. 5.9. It was found that the shape of the CLV curve is very sensitive to the parameters of the model and above all to the resolution of the observations. Convolution with a smearing function not only systematically reduces the contrast but also changes the shape of the CLV, and particularly can change the position of the maximum of the intensity contrast. We found that the position of the maximum of the continuum intensity contrast for the adopted model is in the range of $\mu=0.4-0.7$ depending on the free parameters and the spatial resolution.

The model with $R_{0}=100 \mathrm{~km}$ (Fig. 5.9, left panels) results in intensity contrasts of $20-30 \%$ near the limb which are approximately two times weaker than the observed values. The model with $R_{0}=150 \mathrm{~km}$ produces contrasts closer to the observed. The increase of the number of MFTs (from N2 to N3) along the rays in the resolution element moves the position of the maximum towards the limb and leads to a substantial enhancement of the apparent brightness near the limb, though the contrast at disk center $(\mu=1.0)$ is less sensitive to this parameter of the model (Fig. 5.9, right panel).

Comparing the simulated CLV with the observed ones we can conclude that the model with (HC1, $R_{0}=150 \mathrm{~km}, \mathrm{~N} 3$ ) results in properties, which are closest to the observed. However, it is important to note that although the CLV of the synthetic PF intensities demonstrates trends similar to the observed, no perfect correspondence to the observations was achieved. This can be due to the fact that our model is limited to two components, internal and external, with the external atmosphere prescribed by the VALC model. More realistic calculations of the CLV of the contrast should take into account more components of the photosphere (granule, intergranule, facula interior and exterior atmospheres). 


\subsection{Apparent magnetic field strength}

As it was shown in Sect. 3.3.4 several calibration techniques can be used for an estimation of the apparent magnetic field strengths in solar facular. These approaches are based on several assumptions which simplify the interpretation of the observations and allow to estimate physical quantities without involving complicated radiative transfer calculations. Yet, the question whether the adopted assumptions are valid for the observations near the limb, where the magnetic field is very intermittent along the LOS, still remains open.

The performed modeling makes it possible to verify the reliability of the used calibrations and to test the validity of the underlying assumptions. The parameters of the used model are: $T_{\text {int }}=\mathrm{HC} 1, R_{0}=150 \mathrm{~km}, B_{0}=1400 \mathrm{G}$ at $z=0 \mathrm{~km}$, distribution N3, $\mu=\cos \theta=0.4$, spatial and spectral resolution of the Fabry-Perot spectrometer in the VTT (narrowband). The output from the model calculations are the synthetic data cubes $I\left(x^{\prime}, y^{\prime}, \lambda\right)$ and $V\left(x^{\prime}, y^{\prime}, \lambda\right)$, where $x^{\prime}$ and $y^{\prime}$ are coordinates in the plane perpendicular to the LOS. In this section we apply exactly the same methods to the synthetic data cubes as were applied to the observed data sets (Sect. 3.3.4).

First, we compare the relation between separations and amplitudes of synthetic Stokes $V$ lobes with the observed relation. The simulated diagram of $\Delta \lambda_{V} v s V_{a m p}$ (right panel of Fig. 5.10) is in very good agreement with the observations from PFs presented in Fig. 3.11. The ranges of the calculated and measured values and the triangular shape of the relation between the separations and amplitudes are very similar.

In order to estimate the apparent magnetic field strengths from the synthetic profiles we have used two approaches:

- from the amplitudes of Stokes $V$ in the weak field approximation (WFA);

- from the separations of the Stokes $V$ extrema strong field regime;

In the weak field approximation the magnetic field was estimated from the synthetic polarimetric signal in all three spectral lines used for the observations (Fig. $5.10 \mathrm{left}$ panel) applying Eq. 3.7. The obtained values are much smaller than the intrinsic kiloGauss fields of the model, because the method gives only the LOS component of the average magnetic flux density $B_{\mathrm{WFA}}=f B \cos \gamma$. The values obtained from the synthetic $\lambda 6149.3$ and $\lambda 6301.5$ lines are close to each other and to the values obtained from the observation with the Fabry-Perot spectrometer in the VTT. As expected, for the $\lambda 6302.5$ the WFA approximation is not at all valid for PFs, this line is very sensitive to the magnetic field, it enters the strong field regime already for 1 kilo-Gauss fields, and Stokes $I$ exhibits non-negligible Zeeman broadening.

The histogram of the magnetic field strength derived from separations of the synthetic Stokes $V$ lobes in the Fe I 6302 line assuming the strong field regime (Fig. 5.10 middle panel) is in good agreement with the real field strengths adopted in the numerical model $\left(B_{0}=1400 \mathrm{G}\right)$. Also the shape of the curve and the range of field strengths are similar to the distribution of the magnetic field strengths measured in PFs on the Sun (Fig. 3.10). We conclude that this method is less sensitive to the area filling factor and inclination of the magnetic field. However, this technique tends to overestimate the field strength. Some $V$ profiles have very large separations, resulting in a long tail of the distribution in the middle panel of Fig. 5.10 which reaches apparent fields of 2 kilo-Gauss, strengths which are not present in the adopted model at the heights of formation of this spectral line. This peculiarity was noted also in the observational data (Sect. 3.3.4, Fig. 3.10). 

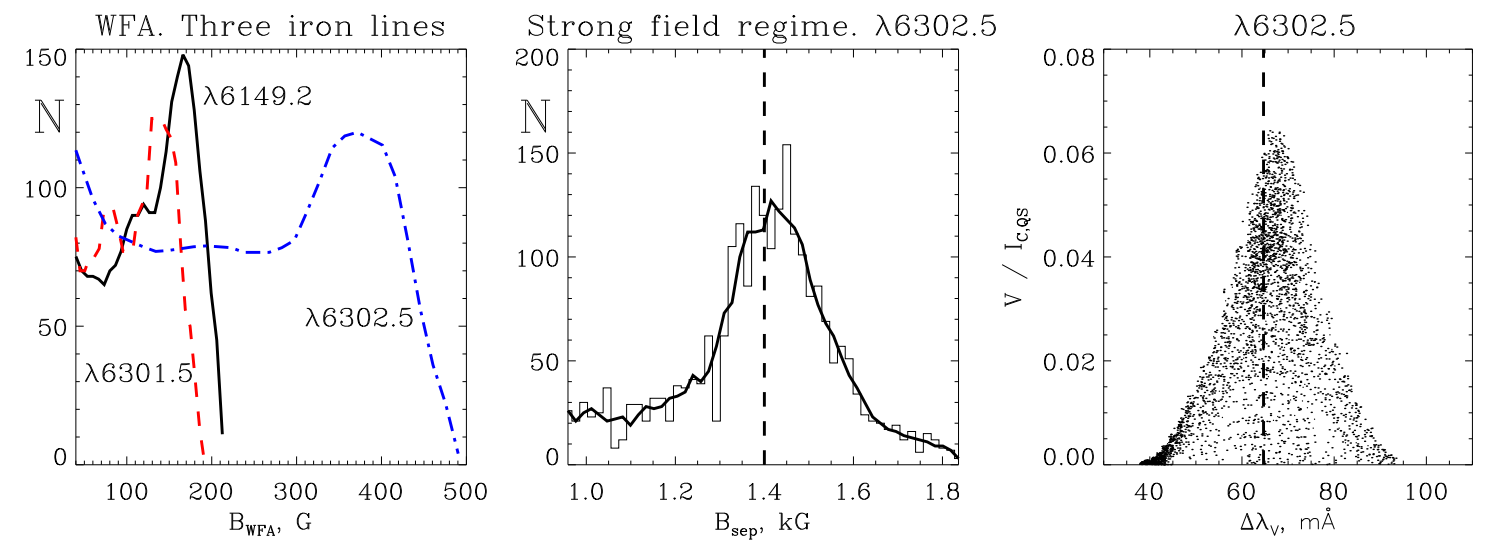

Figure 5.10: Estimation of the apparent magnetic field form the synthetic data cubes $I\left(x^{\prime}, y^{\prime}, \lambda\right)$ and $V\left(x^{\prime}, y^{\prime}, \lambda\right)$. From left to the right: histogram of the average flux density calculated from the WFA; histogram of magnetic field strength calculated from the separations of the $V$ extrema; diagnostic diagram: amplitudes $v s$ separations. The value of the true intrinsic magnetic field (parameter of the model $B_{0}=1400$ kilo-Gauss) is shown by the vertical dashed line.
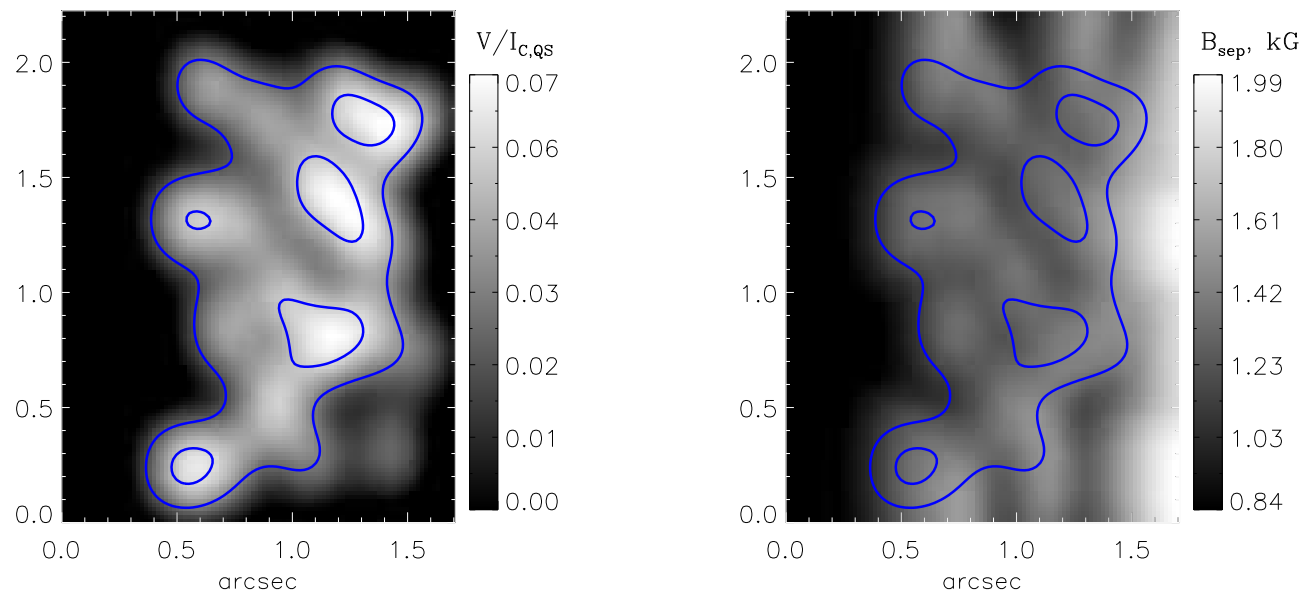

Figure 5.11: Left panel: Amplitudes of the synthetic Stokes $V$ signal. Right panel: Apparent magnetic field measured from the separation of the $V$ lobes. Both maps are overplotted with the contours of the continuum brightness.

A close inspection of the model allows to explain this effect. Magnetograms calculated from the amplitudes and the separations have very different spatial distributions as shown in Fig. 5.11. Note that in this figure the primary information, the magnetic field signals, are given in grey scale, while the intensity pattern showing the location of the facula is overlaid by the contours. The Stokes $V$ amplitudes, apart of the above mentioned small shift towards the center, coincide in general with the intensity pattern, but the separations do not. The most strongly separated $V$ profiles originate from the extreme 
center side of the simulated pattern. They correspond to the special case when the core of a spectral line is formed in a non-magnetic atmosphere, while the intensities at spectral position close to the continuum emerge from the magnetic plasma. The corresponding Stokes $V$ profiles will have no signal at wavelengths close to the line center, and will have weak lobes near the continuum of the spectral line. In such a way, the wide separation of the $V$ lobes does not reflect a strong magnetic field at the heights of formation, but is simply a consequence of the intermittent magnetic field along the LOS.

The most separated $V$ profiles are characterized by very weak $V$ signal, which is below the noise level and therefore they are not detected. However, slightly less separated profiles have accordingly stronger $V$ amplitudes which can be detected. Such profiles result in long tails of the observed distributions (Fig. 3.10).

In this way from the comparison of the properties of the observed and calculated polarimetric signals and of apparent magnetic field found in synthetic and observed faculae we conclude that:

1. the created numerical model with the adopted set of free parameters adequately simulates the properties of the Stokes $V$ signal, apparent magnetic field strengths and average flux density of polar faculae on the Sun, which confirm the reliability of the model;

2. magnetic field determination close to the limb in WFA is valid when applied to proper spectral lines with $\Delta \lambda_{D}>>\Delta \lambda_{V}$, yet this method estimates only the average magnetic flux density in the LOS direction, which strongly depends on the spatial resolution of the observations and on the inclination of magnetic field;

3. magnetic field determination assuming the strong field regime gives reasonable values of magnetic field strength, it is less sensitive to inclinations and area filling factor, however it should be applied with some care to data obtained near the solar limb. This method can overestimate the real field strength when radiation emerges from atmospheres which are magnetically inhomogeneous along the LOS.

\subsection{Summary of results from modeling}

In this section we briefly summarize the main results from the numerical simulations. A realistic numerical model of facula structures observed at large heliocentric angles was created. The model has two components: small-scale magnetic flux tubes embedded in a field free plasma, and is controlled by several free parameters. The magnetic intermittency of the facula atmosphere along the LOS was taken into account consistently.

By varying the free parameters of the model a good agreement of the characteristics of synthetic faculae to the characteristics of the observed polar faculae was achieved.

The created numerical model successfully reproduces the following quantative and qualitive properties of radiation from PFs:

$\star$ enhanced intensity contrasts of facular structures at the limb;

$\star$ fine structure in brightness and magnetic fields; 
$\star$ sizes and shapes of PFs;

$\star$ spatial distribution of apparent magnetic field in PF: maximum of polarimetric signal is shifted towards the center with respect to facular brightenings in continuum;

$\star$ trends and general properties of the center-to-limb variation of continuum intensity contrast and Stokes $V$ amplitude;

$\star$ peculiar spectropolarimetric behavior in all spectral lines under study: depressions and line core contrast in Stokes $I$ profiles and amplitudes and separations of $V$;

$\star$ apparent kilo-Gauss magnetic field strength and average flux density estimated in weak field approximation;

$\star$ model reproduces and allows to explain the observed widely separated Stokes $V$ lobes with $\Delta \lambda_{V}>90 \mathrm{~m} \AA$.

We summarize the obtained parameters of the numerical model, which produce the similarities listed above with observations:

- A large number of magnetic flux tubes along the LOS is required to reproduce the observed intensity contrasts and amplitudes of polarimetric signal. The distribution $\mathrm{N} 3\left(N_{\mathrm{MFT}}=21\right.$ in the simulation box, $f_{\mathrm{mod}}=0.2$, Sect. 4.1.5) was adopted.

- A magnetic field strength $B_{0}=1400 \mathrm{G}$ at $z=0 \mathrm{~km}$ was found to be an adequate choice of this free parameter of the model and it was used throughout the study. This value is in good agreement with the result of the inversion of Stokes profiles from plage regions performed by Bellot Rubio et al. (2000).

- The radius of a MFT at the surface $R_{0}$ was chosen to be in the range $100-150 \mathrm{~km}$. Such values are a compromise between the required large enough contribution from optically thin MFT to the emergent intensities and the fine structure of facula elements.

- Spectral and photometric properties of radiation are very sensitive to the adopted thermal model of the flux tube interior. Starting with the initial temperature stratifications (hot wall and hot cloud) and comparing the synthetic intensity profiles with the observed ones in three iron lines, we have deduced a temperature model (HC1) with $T_{\text {int }}(z<120)<T_{\mathrm{VALC}}$ and $T_{\text {int }}(z>120)>T_{\mathrm{VALC}}$, which results in a good agreement of synthetic profiles with observations in all three lines simultaneously. This temperature stratification of flux tube interior agrees well with results of inversion of Stokes profiles (Bellot Rubio et al. 2000). Thus, the deduced model is an appropriate choice of temperature stratification inside MFTs for the performed simulations with the adopted model assumptions, i.e. two components and fixed parameters of the external atmosphere. 


\section{Conclusions and outlook}

In this work the structure and dynamics of polar faculae on the Sun, which constitute an important part of the magnetic solar cycle, were studied by means of high resolution observations and numerical simulation.

Sophisticated methods of image processing and data analysis were applied to the observations, which allow to improve the spatial resolution of images (Sects. 2.2.4 and 2.2.5) and to achieve better spectropolarimetric sensitivity (Sect. 2.3). The application of such methods allows to detect a wealth of substructure and dynamic phenomena of PFs. The summary of the obtained observational properties of polar faculae is given in Sect. 3.6. Here we recall that PFs were found to be highly structured phenomena, characterized by kilo-gauss magnetic fields and very dynamical behavior.

Strong magnetic fields and unipolarity which were found in PFs confirm the concept that polar faculae belong to the global poloidal magnetic field of the Sun.

The unexpected discovery of systematic upflows in PFs points at the possible relation of photospheric faculae at high latitudes of the Sun to the polar coronal holes as sources of the fast solar wind from the polar caps. It should stimulate the further search of the connections between the small-scale magnetic elements in the solar photosphere and the coronal structures and solar wind.

The presented study on PFs needs further observational confirmation and should be compared with data from equatorial faculae when observed also near the limb. The observations which form the basis of this study were obtained in the years 2001-2002 close to the maximum of the sunspot cycle, and accordingly PFs were rare at the solar poles at that time. Therefore, observations of polar activity during minimum of the sunspot cycle will allow to obtain better statistics. Moreover, systematic high resolution spectropolarimetric observations of PFs during the whole solar activity cycle are desirable. It will help to understand better the connection of polar faculae to the global magnetism of the Sun.

Finally, we note that for the successful study of small-scale magnetic structures close to the limb the full four-component Stokes vector should be measured and observational facilities which combine high spectral and spatial resolution with a high spectropolarimetric sensitivity and low instrumental polarization are required.

The second part of the study consists of numerical simulations of small-scale magnetic structures close to the solar limb aiming at the reproduction of the observed properties of PFs. Thus, a numerical model was constructed and radiative transfer calculations and spectral line synthesis were performed. In Sect. 5.7 the results of the simulations are summarized. We note, that the accurate treatment of the magnetic intermittency of facula atmospheres allows to simulate successfully many of the characteristics of PFs of the Sun. 
The ability of the adopted numerical model with the reasonable choice of parameters to reproduce and explain the observed properties of polar faculae proves the reliability of the hypothesis that PFs consist of concentrations of small-scale kilo-Gauss magnetic flux tubes.

The constructed model is controlled by several free parameters and therefore can be used for interpretation of the polarimetric observations at large heliocentric angles and for verifications of different calibration techniques used for data analyses.

For the sake of simplicity the model was limited to the two-component (internal and external) case with static magnetic flux tubes and zero macroscopic velocities. Therefore, the evident improvement of the model would be in abandoning the above simplifications:

- radiative transfer calculation in the simulation box with a predefined 3D velocity field will allow to simulate in a realistic way the asymmetries of Stokes $I$ and $V$ profiles and upflows found in PFs;

- a model with more components (granule, intergranule, faculae interior and exterior atmospheres) should provide better correspondence of individual synthetic intensity profiles to the observed ones and also improve the synthetic center-to-limb variation of the continuum intensity contrast;

- selfconsistent dynamical model to simulate the time evolution of PFs.

The two latter improvements can be achieved only by combination of realistic magnetohydrodynamic simulations with radiative transfer calculations at large heliocentric angles. 


\section{Bibliography}

Allen, C.W.: 1976, Astrophysical Quantities, 3rd ed., The Athlone Press, University of London

Aller, L.H.: 1963, Astrophysics. The Atmospheres of the Sunand Stars, 2nd ed., Ronald Press, New-York

Audic, S.: 1991, Solar Phys., 135, 275

Auffret, H., Muller, R. 1991, A\&A, 246, 264

Babin, A.N., Gopayuk, S.I., Efanov, V.A., et al.: 1976, Izv. Krymsk. Astrfiz. Obs., 55, 3

Beckers, J.M., Schröter, E.H.: 1968, Solar Phys., 4, 142

Bellot Rubio, L.R., Ruiz Cobo, B., Collados, M.: 2000, ApJ, 535, 489

Bendlin, C., Volkmer, R.: 1995, A\&AS, 112, 371

Bendlin, C., Volkmer, R., Kneer, F.: 1992, A\&A, 257, 817

Berger, T.E, Lödfahl, M.G., Shine, R.A., Title, A.M.: 1998, ApJ, 495, 973

Borrero, J.M., Bellot Rubio, L.R.: 2002, A\&A, 385, 1056

Brault, J.W., Neckel, H.: 1987 Spectral Atlas of Solar Absolute Disk-Averaged and Diskcenter Intensities from 3290 to $12510 \AA$

Brault. J.W., White, O.R.: 1971, A\&A, 13, 169

Bünte, M., Solanki, S.K., Steiner, O.: 1993, A\&A, 268, 736

Capitani, C., Cavallini, F., Ceppatelli, G., et al.: 1989, Solar Phys., 120, 173

de Boer, C.R.: 1993, PhD thesis, Göttingen University

de Boer, C. R.: 1996, A\&AS, 120, 195

DeForest, C. E., Hoeksema, J. T., Gurman, J. B., et al.: 1997, Solar Phys., 175, 393

Deinzer, W., Hensler, G., Schüssler, M., Weisshaar, E.: 1984a, A\&A, 139, 426

Deinzer, W., Hensler, G., Schüssler, M., Weisshaar, E.: 1984b, A\&A, 139, 435

Denskat, K.U.: 1982, PhD thesis, Braunschweig University

Dikpati, M., de Toma, G., Gilman, P.A., Arge, C.N., White, O. R.: 2004, ApJ, 601, 1136

Domínguez Cerdeña, I., Kneer, F., Sánchez Almeida, J.: 2003, ApJ, 582, L55

Efanov, V.A., Labrum, N., Moiseev, I.G., et al.: 1980, Izv. Krymsk. Astrfiz. Obs., 61, 52 
Eker, Z.: 2003, Solar Phys., 212, 277

Fabiani Bendicho, P., Kneer, F., Trujillo Bueno, J.: 1992, A\&A, 264, 229

Gurtovenko, E., Ratnikova, V., De Jager, C.: 1974, Solar Phys., 37, 43

Hasan, S.S., Kneer, F., Kalkofen W.: 1998, A\&A, 332, 1064

Hofmann, A.: 2000, private communication

Homann, T., Kneer, F., Makarov, V.: 1997, Solar Phys., 175, 81

Janssen, K.: 2003, PhD thesis, Göttingen University

Joliffe, I. T.: 1986, Principal Component Analysis, Springer, New York

Karpinsky, V., Okunev, O.: 1997, Solar Phys., 173, 232

Karpinsky, V., Okunev, O.: 1998, Solar Phys., 183, 277

Keller, C.U., von der Lühe, O.: 1992, A\&A, 261, 321

Kentischer, T.J., Schmidt, W., Sigwarth, M., von Uexküll, M.: 1998, A\&A, 340, 569

Kneer, F., Hirzberger, J.: 2001, Astron. Nachr./AN, 322, 375

Kneer, F., von Uexküll, M.: 1991, A\&A, 247, 556

Knölker, M., Schüssler, M., Weisshaar, E.: 1988, A\&A, 194, 257

Koschinsky, M.: 2001, PhD thesis, Göttingen University

Koschinsky, M., Kneer, F., Hirzberger, J.: 2001, A\&A, 365, 588

Krat, V. A., Karpinsky, V. N., Pravdjuk, L. M.: 1972, Solar Phys., 26, 305

Krieg, J.: 1999, PhD thesis, Göttingen University

Krieg, J., Wunnenberg, M., Kneer, F., Koschinsky, M., Ritter, C.: 1999, A\&A, 343, 983

Landi Degl'Innocenti, E.: 1976, A\&AS, 25, 379

Landi Degl'Innocenti, E.: 1992, in F. Sánchez, M. Collados, and M. Vázquez (Eds.), Solar Observations: Techniques and Interpretation, First Canary Islands Winter School, Cambridge Univ. Press, Cambridge UK, p.77

Lawrence, J. K., Chapman, G. A.: 1988, ApJ, 335, 996

von der Lühe, O.: 1984, J. Opt. Soc. Am. A1, 510

von der Lühe, O., Schmidt, W., Soltau, D., Berkefeld, Th., Kneer, F., Staude, J.: 2001, Astron. Nachr./AN, 322, 353

Magain, P.: 1986, A\&A, 163, 135

Makarov, V. I., Sivaraman, K. R.: 1989, Solar Phys., 123, 367

Makarov, V.I., Makarova, V.V., Bogod, V.N., et al.: 1991, Soln. Dann. 9, 87

Makarov, V.I., Makarova, V.V.: 1996, Solar Phys., 163, 267

Makarov, V.I., Tlatov, A.G., Sivaraman, K. R.: 2003, Solar Phys., 214, 41

Mehltretter, J.P., Sterne und Weltraum, 1976, vol. 15, p. 44-47 
Muller, R., Rouder, T.: 1984, Solar Phys., 94, 33

Neckel, H.: 1999, Solar Phys., 184, 421

Okunev, O., Kneer, F.: 2004, A\&A, 425, 321

Ortiz, A., Solanki, S. K., Domingo, V., Fligge, M., Sanahuja, B.: 2002, A\&A, 388, 1036

Ossedrijver, M.: 2003, A\&A Rev., 11, 287

Pérez Rodríguez, E., Kneer, F.: 2002, A\&A, 395, 279

Pneuman, G.W., Solanki, S.K., Stenflo, J.O.: 1986, A\&A, 154, 231

Puschmann, K., et al.: 2004, A\&A, in press

Rachkovsky, D.N.: 1962, Izv. Krym. Astrof. Obs., 28, 259

Rees, D.R., Murphy, G.A, Durrant, C.J.: 1989, ApJ, 339, 1093

Riehokainen, A., Urpo, S., Valtaoja, E.: 1998, A\&A, 333, 741

Riehokainen, A., Urpo, S., Valtaoja, E., Makarov, V. I., Makarova, L. V., Tlatov, A. G.: 2001, A\&A, 366, 676

Ritter, C.: 2001, PhD thesis, Göttingen University

Ruiz Cobo, B., del Toro Iniesta, J. C.: 1992, ApJ, 398, 375

Sánchez Almeida, J., Martínez Pillet, V., Wittmann, A.: 1991, Solar Phys., 134, 1

Sánchez Almeida, J., Martínez Pillet, V.: 1994, ApJ, 424, 1014

Sánchez Cuberes, M., Vázquez, M., Bonet, J.A., Sobotka, M.: 2002, ApJ, 570, 886

Schmidt, W., Solanki, S.K., Lites, B.W., Title, A.M., Martínez Pillet, V.: 2001, Astron. Nachr./AN, 322, 363

Schröter, E.H.: 1956, ZAp, 41, 141

Shchukina, N., Trujillo Bueno. J.: 2001, ApJ, 550, 9706

Sheeley, N.R., Jr.: 1964, ApJ, 140, 731

Sheeley, N.R., Jr.: 1991, ApJ, 374, 386

Solanki, S.K.: 1993, Space Sci. Rev., 63, 1

Solanki, S.K., Steiner, O., Bünte, M., Murphy, G., Ploner, S.R.O: 1998, A\&A, 333, 721

Soltau, D.: 1997, A\&A, 317, 586

Spruit, H.C.: 1976, Solar Phys., 50, 269

Spruit, H.C.: 1977, PhD thesis, Utrecht University

Stenflo, J.O.: 1973, Solar Phys., 32, 41

Stenflo, J. O.: 1994, Solar Magnetic Fields - Polarized Radiation Diagnostics (Kluwer Academic Publ.)

Stix, M.: 2002, The Sun. An Introduction, 2nd ed., Berlin Springer

Stolpe, F., Kneer, F.: 1998, A\&AS, 131, 181 
Thévenin, F.: 1990, A\&AS, 82, 179

Unno, W.: 1956, Publ. Astron. Soc. Japan, 8, 108

Unsöld, A: 1955, Physik der Sternatmosphären, 2nd ed. (Springer, Heidelberg), 333

Vernazza, J.E., Avrett, E.H., Loeser, R.: 1981, ApJS, 45, 635

Vögler, A., Schüssler, M.: 2003, Astron. Nachr./AN, 324, 399

Volkmer, R.: 1995, PhD thesis, Göttingen University

Waldmeier, M.: 1955, ZAp, 38, 37

Waldmeier, M.: 1962, ZAp, 54, 260

Wang, Y.M., Sheeley, N. R., Jr.: 2003, ApJ, 599, 1404

Weigelt, G.P.: 1977, Optics Comm. 21, 55

Wittmann, A.: 1974, Solar Phys., 35, 11

Wilhelm, K., Marsch, E., Dwivedi, B. N., et al.: 1998, ApJ, 500, 1023

Wilhelm, K., Dammasch, I. E., Marsch, E., Hassler, D. M.: 2000, A\&A, 353, 749

Zhang, M., Zhang, H. O.: 1999, A\&A, 352, 317

Zirin, H.: 1999, Solar Phys., 184, $249 \%$ 


\section{Acknowledgements}

The work on my doctoral thesis was carried out in the Universitäts-Sternwarte, a scientific institution within the physics faculty of the Georg-August-Universität in Göttingen.

I would like to thank the Universitäts-Sternwarte for the hospitality and for its unique scientific atmosphere. Many thanks to my supervisor Franz Kneer for his patience and for the guidance and support during my entire $\mathrm{PhD}$ study. I would like to express my gratitude to him for being always available and ready to help and to give a good advice, discussions with him and his suggestions on different topics of the project were always very helpful.

I am very grateful to the people without whom my $\mathrm{PhD}$ would not have been possible: to Dr. Vadim Karpinsky, who was my first supervisor at the Central (Pulkovo) Astronomical observatory in St. Petersburg and to Dr. Larissa Pravdjuk, who was always encouraging, supporting and helping me.

Financial support by Deutscher Akademischer Austaushdienst (DAAD) through grant A/00/01395 is gratefully acknowledged.

An important part of the project was observations with the German solar telescopes at Tenerife. The Gregory-Coudé Telescope had been operated by the Universitäts-Sternwarte Göttingen at the Spanish Observatorio del Teide of the Instituto de Astrofísica de Canarias. It was dismounted in spring 2002. The Vacuum Tower Telescope is operated by the Kiepenheuer-Institut für Sonnenphysik, Freiburg, at the Observatorio del Teide.

During my PhD study I was participating in the seminars and other events of the International Max Plank Research School on Physical Processes in the Solar System and Beyond, for which I would like to thank the coordinator of the school Dr. D. Schmitt.

I wish to express my gratitude to all my friends, whom I have met in Göttingen and in Germany, for the nice time we spent together during the years of my study. This time I will never forget.

Finally, I thank my family, and above all my mother for the love, care and support which I have been feeling even being so far from my home. 



\section{Lebenslauf}

Name: $\quad$ Oleg Okunev

Geburtsdatum: $\quad 08.02 .1974$

Geburtsort: $\quad$ Sankt Petersburg, Russland (damals Leningrad, UdSSR)

Familienstand: $\quad$ ledig

Eltern: $\quad$ Victor Okunev

Lidia Okuneva

Staatsangehörigkeit: russisch

Schulbildung: Septermber 1981 - Juni 1991 Schule N61 in Sankt Petersburg

Studium: $\quad$ September 1991 - Juni 1997 Technische Staats-Universität in Sankt Petersburg, Fakultat der Physik und Technik, Abteilung für Weltraumphysik

$1996-1997$ Diplomarbeit am Haupt-Astronomischen Observatorium in Pulkovo zum Thema: "Numerische Simulationen photosphärischer Strukturen am Sonnenrand"

Juni 1997 Diplom

Beruf: $\quad$ Juli 1997-Juli 2000

Wisseschaftlicher Mitarbeiter an der HauptAstronomischen Observatorium in Pulkovo

Promotion: Oktober 2000 - August 2004 Promotion an der Universitäts-Sternwarte, Göttingen

Oktober 2000 - Oktober 2003 Stipendium des Deutschen Akademischen Austauschdienstes (DAAD) A/00/01395 